\title{
SISMES X NATIONAL CONGRESS
}

\author{
Messina, 5-7 October 2018
}

Springer-Verlag Italia S.r.1., part of Springer Nature 2018

\section{MARCELLO FAINA AND ARSENIO VEIC- STEINAS LECTURE}

\section{Exercise training: the maintenance of neuromuscular function in the older individual}

\author{
G. De Vito \\ School of Public Health, Physiotherapy and Sports Science, \\ University College Dublin, Ireland
}

\begin{abstract}
According to the most recent projections the number of older European citizens (65+ years) will increase from 87 million in 2010 to 148 million in 2060. An augmented life expectancy is the reflection of the many societal successes we experienced in the last 4-5 decades in terms of medical care and improved living conditions. In this context, it is of vital importance to maintain and improve both health and independence in the entire life span. The concept of independence includes the ability to remain mobile and to perform competently the activities of daily living, such as: raising from a chair, climbing stairs or carrying and lifting shopping bags, all actions requiring adequate level of muscle strength, cardio-respiratory fitness and of postural balance and flexibility.

Aging unfortunately, is associated with a generalized deterioration of physiological function including a progressive decline in skeletal muscle mass and muscular strength and power which progressively translate into functional impairment, increased rate of disability and dependency. It is now unanimously recognized that the practice of regular physical exercise is one of the most powerful forms of nonpharmacological therapeutic and preventive intervention. Progressive Resistance training (PRT) is presently recommended as an essential component of a broad-spectrum fitness program and has been clearly shown effective for both strength and power enhancement in older individuals. However, PRT may not be optimal for all functional outcomes and, more importantly, may not represent the first choice of exercise for the over 65's, especially women, who may prefer other kind of activities. Indeed, previous investigations have reported that multicomponent training (MCT) focusing on neuromuscular coordination and combining endurance and muscle strength training components could be similarly effective in reducing the development and progression of chronic diseases and disabling conditions.
\end{abstract}

In this presentation, therefore, I will illustrate the results of some studies conducted in the last 20 years which adopted either PRT or MCT. In addition, the importance of a proper nutritional support will be discussed underlying the importance of maintaining an adequate protein intake for the older individuals regularly engaged in exercise training. I will also briefly present the results concerning the effects of PRT on markers of muscle atrophy/hypertrophy and heat shock response (HSR) in healthy older participants of both genders.

\section{INVITED LECTURE}

\section{Adipose organ and physical exercise}

\section{S. Cinti}

Università Politecnica delle Marche

Most of white and brown adipocytes, in spite of their different functions: storing energy and thermogenesis, are contained together in a true organ formed by subcutaneous and visceral depots. The reason for this mixture resides in the fact that adipocytes have plastic properties allowing them to convert each other: under chronic cold exposure white convert into brown to support the need for thermogenesis and under obesogenic diet brown convert into white to satisfy the need for energy storing.

The white-brown transdifferentiation (WAT browning) is of medical interest because the browning is associated with obesity resistance and drugs inducing the browning curb obesity and related disorders.

Type 2 diabetes is the most common disorder associated to visceral obesity. Macrophages infiltrating the obese adipose organ are responsible for the low-grade chronic inflammation dealing to insulin resistance and T2 diabetes. Macrophages form characteristic histopathology figures: crown like structures (CLS) due to the need of removal debris deriving from the death of adipocytes. Death of adipocytes is related to their hypertrophy up to the critical death size. Visceral adipocytes have a smaller critical death size, thus offering an explanation for the higher inflammation and morbidity of visceral fat. Physical exercise induces size reduction and mitochondrial biogenesis in adipocytes of white adipocyte. This well-known observation can 
now be viewed as an early step of WAT browning and several healthy consequences of physical exercise can be attributed to this phenomenon. The mechanisms involved include the activity of several actors. Central nervous system acts with a direct increase of activity and with expansion of its sympathetic branch. Interestingly, the same amount of physical exercise induces more browning in animals maintained in an enriched environment. In these animals, the increased BDNF hypothalamic production seems to be responsible for the enhanced browning. Several cytokines or myokines produced by active skeletal muscles have been suggested to play a role in WAT browning. In 2012 it has been showed that transgenic muscles engineered to mimic trained muscles upregulated the gene expression of fibronectin type III domain containing 5 (FNDC5), which after cleavage is secreted into the blood stream as irisin. Natriuretic peptides are hormones produced by heart with the main purpose to maintain homeostasis with regard to blood volume, blood pressure, and salt balance. The adipose organ influences the activity of these peptides by clearance receptors. On the other hand, natriuretic peptides acting on functional receptors increase cyclic GMP levels to activate cGMP-dependent protein kinase and activation of p38MAPK, thus their activity could be synergic with that of classic $\beta$-adrenergic receptor stimulation. It has been showed that natriuretic peptides promote browning of human white adipocytes and browning in treated mice. IL-6 is produced by skeletal muscles and other organs (including fat). Its production increase during exercise and in its overexpression in mice increase BAT activity. Recently a WAT browning effect of IL-6 has also been proposed. Exercised skeletal muscles also secrete the myokine $\beta$-aminoisobutyric acid (BAIBA). BAIBA induces WAT browning and $\beta$-oxidation in hepatocytes both in vitro and in vivo. Interestingly, in a large human cohort study (Community-based Framingham Heart Study), plasma BAIBA concentrations resulted increased with physical exercise and inversely associated with metabolic risk factors. Meteorin-like (Metrnl) is a circulating factor that is induced in muscle after exercise and in WAT after cold exposure. Increasing serum levels of Metrnl stimulates WAT browning. Metrnl stimulates an eosinophil-dependent increase in IL-4 expression and promotes alternative activation of adipose tissue macrophages, which are required for the increased expression of the thermogenic gene programs in fat. Finally explanted subcutaneous fat of trained mice into sedentary animals improved their glucose tolerance and glucose uptake in muscle suggesting the possibility that trained subcutaneous fat could release adipokines that could reinforce the healthy WAT browning phenomenon.

\section{SESSIONS}

\section{ABBREVIATIONS SESSIONS}

$\mathrm{TP}$

$\mathrm{EH}$

EP

PE

SB

$\mathrm{BN}$

AP

SP

ML

$\mathrm{PO}$

MS

SEL
Training performance and evaluation methods

Physical exercise as prevention and therapy

Exercise physiology

Physical education and sport pedagogy

Sport biomechanics and technology

Biomolecular and nutritional aspects of exercise and sports

Adapted physical activity

Exercise and sport psychology

Movement learning and psychophysiological development

Postural approach to sport and exercise

Morphological sciences in sport and exercise

Social, economic and legal sport's aspects

\section{PRESENTATION TYPE}

K Keynote oral presentation

O Oral presentation

P Poster

\section{FRIDAY ORAL SESSION}

\section{TRAINING, PERFORMANCE AND EVALUATION METHODS 1}

\section{TP1 K}

Assessment of work load intensity in respiratory muscle training

\author{
C. Doria ${ }^{1,2}$, E. Ce $e^{, 1,2}$, S. Longo ${ }^{1}$, A. Montaruli ${ }^{1}$, \\ G. Coratella ${ }^{l}$, E. Limonta ${ }^{l}$, S. Shokohyar ${ }^{l}$, \\ S. Rampichini ${ }^{1,2}$ F. Esposito ${ }^{1,2}$
}

${ }^{1}$ Department of Biomedical Sciences for Health, University of Milan; ${ }^{3}$ Interuniversity Institute of Myology, Italy

Purpose: The respiratory muscles can be trained like the other skeletal muscles. However, due to the complexity of the thoracopulmonary system, the assessment of work load intensity during training still remains a complicated matter. Therefore, the aim of this study was to evaluate the feasibility of work load intensity quantification during a period of respiratory muscle training.

Methods: Seven healthy participants performed a four-week respiratory muscle training under normocapnic hyperpnoea using a device that allows a constant isocapnic end-tidal $\mathrm{CO}_{2}$ fraction via a two-way piston valve connected to a rebreathing bag. The size of the bag was adjusted to $50-60 \%$ of the subject's vital capacity, with a breathing frequency such that requested ventilation corresponded to $50-60 \%$ of maximal voluntary ventilation. Participants trained about 24 min per session ( $5 \mathrm{~min}$ of exercise/ $1 \mathrm{~min}$ of rest, 4 times), 3 days a week, for 4 weeks. The isocapnic device estimated the entire breath-volume during training. At the end of each training session, we asked to provide a rating of the perceived exertion (sRPE, a.u.; CR-10 scale). Results: A moderate correlation between the entire breath-volume during training and the sRPE was found in all training sessions $(\mathrm{r}=0.54 ; \mathrm{p}<0.01)$, while moderate to very strong correlations were found for each participant $(r=0.58 ; p<0.05-r=0.96 ; p<0.01)$.

Conclusions: The findings of this study suggest that the sRPE, a cost-effective monitoring tool, could be a valid approach to assess training work load during respiratory muscle training. 


\section{TP1 O}

\section{Fitness and hormonal modifications after pre-season training in youth soccer players}

\author{
L. Innocenti ${ }^{1}$, F. Perroni $^{2}$, S. Fittipaldi ${ }^{3}$, L. Falcioni ${ }^{1}$, \\ L. Ghizzoni $^{4}$, S. Migliaccio ${ }^{1}$, L. Guidetti ${ }^{1}$, C. Baldari ${ }^{1,5}$ \\ ${ }^{1}$ Department of Movement, Human and Health Sciences, University \\ of Rome "Foro Italico", Rome, Italy; \\ ${ }^{2}$ Italian Fire Fighter Corp, Rome, Italy; \\ ${ }^{3}$ IRCCS SDN, Napoli, Italy; \\ ${ }^{4}$ Department of Medical Sciences, University of Turin, Turin, Italy; \\ ${ }^{5}$ eCampus University, Novedrate (CO), Italy
}

Purpose: Considering that the cure of the physical preparation poses a heavy physiological and psychological burden on players (Caldwell and Peters 2009) and it is considered as an important element to influence the final result (Reilly 2003), the aim of the present study was to investigate the effect of 8 weeks of pre-season training on salivary steroid hormones and fitness parameters in youth soccer players.

Methods: We recruited 20 male young soccer players (age $14 \pm 0$ years; weight $61.9 \pm 7.9 \mathrm{~kg}$; height $171 \pm 0.1 \mathrm{~cm}$; BMI $21.0 \pm 1.7 \mathrm{~kg} / \mathrm{m}^{2}$ ) that took part at pre-season training phase (one session/day for the first 4 weeks, and 3 training session/week performed for the following 5-8 weeks). Fitness parameters were evaluated by analysing explosive strength by Counter Movement Jump test (CMJ) and resistance by Yo-Yo Intermittent Recovery Test $\left(\mathrm{VO}_{2 \max }\right)$ while, to assess potential modification, hormones salivary samples (CortisolsCand Testosterone-sT) were collected by SaliTubes and assessed by Elisa. Analysis of variance for repeated measures was conducted to assess differences in fitness and hormonal variables with time (i.e., pre-post) and Pearson correlations was used to calculate possible correlation between percentage of variation between preand post value (\% Diff) for each variable.

Results: Decreased value in CMJ (pre $30.5 \pm 4.3 \mathrm{~cm}$; post $28.0 \pm 2.97 \mathrm{~cm} ; \mathrm{p}<0.01$ ) but increased value in $\mathrm{VO}_{2 \max }$ (pre $42.8 \pm 1.9 \mathrm{~mL} / \mathrm{kg} / \mathrm{min}$; post $47.3 \pm 2.1 \mathrm{~mL} / \mathrm{kg} / \mathrm{min}, \mathrm{p}<0.01$ ) were found in the young soccer players. Considering the percentage of variation (\% Diff) of variables, no significant correlation emerged among them. A significant decrease of $\mathrm{sC}$ (pre $6.8 \pm 3.4 \mathrm{pg} / \mathrm{ml}$; post $1.2 \pm 0.7 \mathrm{pg} / \mathrm{ml} ; \mathrm{p}<0.01$ ) and of sT (pre $76.9 \pm 32.9 \mathrm{pg} / \mathrm{ml}$; post $42.8 \pm 25.1 \mathrm{pg} / \mathrm{ml} ; \mathrm{p}<0.05)$ was observed during the training period while a concomitant increased T/C ratio $(\mathrm{p}<0.01)$ was observed, suggesting alteration in stress condition, but unchanged puberty status. Conclusions: Pre-season training phase induced an improvement in $\mathrm{VO}_{2 \max }$ but an impairment in explosive strength. This could be linked to an excessive eccentric load on lower limb that had negative influence only on explosive performance (CMJ). Generally, training loads increase up to 2.4 times in pre-season training phase compared with the in-season phase (Jeong et al. 2011). Our young soccer players, after 8 week pre-season training improved the T/C ratio indicating both a positive anabolic/catabolic balance and buffering against stress.

References

Caldwell BP, Peters DM (2009) Seasonal variation in physiological fitness of a semiprofessional soccer team. J Strength Cond Res 23:1370-1377

Jeong TS, Reilly T, Morton J, Bae SW, Drust B (2011) Quantification of the physiological loading of one week of "pre-season" and one week of "in-season" training in professional soccer players. J Sports Sci 29(11):1161-1166
Reilly T (2003) Motion analysis and physiological demands. In: Reilly T, Williams AM (eds) Science and soccer. Routledge, London, p 59

\section{TP1 O \\ Technical and positional analysis of the Asian Cup in football}

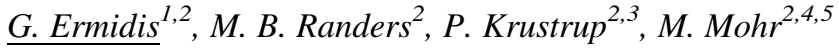

${ }^{1}$ Department of Movement and Wellness Sciences, University of Naples "Parthenope", Naples, Italy;

${ }^{2}$ Department of Sports Science and Clinical Biomechanics, SDU Sport and Health Sciences Cluster (SHSC), Faculty of Health Sciences, University of Southern Denmark, Odense, Denmark; ${ }^{3}$ Sport and Health Sciences, University of Exeter, Exeter, UK; ${ }^{4}$ Center of Health and Human Performance, Department of Food and Nutrition, and Sport Science, University of Gothenburg, Gothenburg, Sweden;

${ }^{5}$ Centre of Health Science, Faculty of Health Sciences, University of the Faroe Islands, Torshavn, Faroe Islands

Purpose: In the last two decades, analyses of sports performance using notational systems, video and new technology have helped coaches and athletes to improve performance (Sarmento 2018). The aim of this study is to identify differences in technical variables between positions of field players.

Methods: 202 top-level soccer players from 16 national teams were monitored during the 2015 Asian Cup. Players were categorised by playing position as central defenders $(C D, n=43)$, full-backs (FB, $\mathrm{n}=41$ ), central midfielders $(\mathrm{CM}, \mathrm{n}=56)$, external midfielders ( $\mathrm{EM}$, $\mathrm{n}=31$ ) and attackers $(\mathrm{FW}, \mathrm{n}=31)$. We analysed only players that played $90 \mathrm{~min}$. Data were collected with Opta Sportsdata ( http://www.optasports.com). Differences in playing positions to different technical variables were analysed using Kruskal-Wallis H nonparametric test.

Results: Goals scored was only significant different between EM and FB, with EM scoring more goals than FB. FB had also significant less attempts than CM, EM, FW, and $\mathrm{CD}$ had less attempts than FW. Ground duels of CD were significant less than FB, CM, EM, FW. CM had more total passes than CD, EM, FW but FW less passes than CD, $\mathrm{FB}$ and $\mathrm{CM}$. CM had more short passes than all positions and FB more short passes than FW. Long passes were significant different between all positions $(\mathrm{CD}>\mathrm{FB}>\mathrm{CM}>\mathrm{EM}>\mathrm{FW})$.

Conclusions: Based on these results, we propose that training should be individually planned to match the technical indicators of different positions. In conclusion, all positions need special attention in accordance with the demands of the position and the need of creating drills for each of them as a part of warm-up, fitness training or technical exercise.

\section{Reference}

Sarmento H et al (2018) Sports Med 48:799-836 


\section{TP1 O}

\section{Half is enough: low volume HIT can improve soccer specific physical capacities}

\section{P. Izzicupo ${ }^{1}$, B. Ghinassi $^{1}$, A. Di Credico ${ }^{1}$, G. Gaggi ${ }^{1}$, A. Zampacorta ${ }^{1}$, Cordellat Ana ${ }^{2}$, A. Di Baldassarre ${ }^{1}$, F.Y. Nakamural}

${ }^{I}$ Department of Medicine and Aging Sciences, University " $G$. D'Annunzio" of Chieti-Pescara;

${ }^{2}$ Department of Sport and Physical Education, University of Valencia

Purpose: This study aimed to compare chronic effects of performing two sets vs. one set of short interval high-intensity intermittent training (HIT) runs on jump, sprint and intermittent endurance performances of semi-professional soccer players during preseason.

Methods: Eighteen male players $(20.1 \pm 3.8$ years $)$ were assessed for sprint (20-m), countermovement jump (CMJ), 30-15 Intermittent Fitness Test (30-15 $\left.{ }_{\mathrm{IFT}}\right)$ at baseline, randomly assigned to 6-8 training sessions, and reassessed after training period. Sprint and CMJ were assessed by an iPhone 6s using MySprint and MyJump 2.0 (Apple Inc., USA) mobile applications, respectively. The $30-15_{\text {IFT }}$ was conducted according to the procedures outlined by Buchheit (2008). Training sessions consisted of: (i) 1 set of 6 sprints (20-m) interspersed with $2 \mathrm{~min}$ of passive recovery, followed by $6-8 \mathrm{~min}$ HIT, comprising alternation of 15 -s runs at $85-95 \%$ of the velocity attained during the $30-15_{\mathrm{IFT}}\left(\mathrm{V}_{\mathrm{IFT}}\right)$ and 15 -s passive rest $(\mathrm{S}+\mathrm{H}$ G) or; (ii) 2 sets of the same HIT protocol, separated by $4 \mathrm{~min}$ of passive rest $(2 \times 6-8$ min of set duration) $(2 \mathrm{H} \mathrm{G})$. Magnitude based-inference analysis was used (1) to examine the changes in $\mathrm{V}_{\mathrm{IFT}}$, sprint and jumps within $\mathrm{S}+\mathrm{H} \mathrm{G}$ and $2 \mathrm{H} \mathrm{G}$ and; (2) to compare between-group changes.

Results: The increase in $\mathrm{V}_{\mathrm{IFT}}$ was almost certain for both groups (ES $=0.83$ and 1.20 , for $S+H$ G and $2 S \mathrm{G}$, respectively), while the differences in the changes between groups was rated as unclear. The changes in CMJ height within each group were unclear, but the $\mathrm{S}+\mathrm{H}$ G presented a likely small decrement in sprint performance $(\mathrm{ES}=0.28)$, which resulted in a possibly small worsening in comparison to $2 \mathrm{H} \mathrm{G}$.

Conclusion: Performing 1 or 2 sets of HIT per training session resulted in similar increase of $\mathrm{V}_{\mathrm{IFT}}$ in semi-professional soccer players. Therefore, low-volume HIT can improve aerobic fitness while preserving jump ability. The addition of sprint training to HIT did not result in improved sprint performance.

\section{Reference}

Buchheit M (2008) The 30-15 intermittent fitness test: accuracy for individualizing interval training of young intermittent sport players. $\mathrm{J}$ Strength Cond Res 22:365-374

\section{TP1 O}

\section{Autogenic training effects on endurance runners performance}

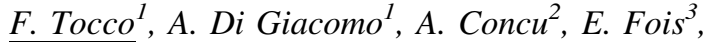 \\ $\overline{\text { P. Mulas }}^{3}$, W. Orru ${ }^{3}$
}

${ }^{1}$ Sports Physiology Laboratory, University of Cagliari, Italy;

${ }^{2}$ C Technologies Inc., Academic Spin-off, University of Cagliari, Italy;

${ }^{3}$ Spitha Sport Counseling, Formist, Cagliari, Italy

Aim: Several sportsmen utilize autogenic training (AT) for the purpose to improve their performance. The aim of this study was to verify the AT effects on several respiratory and metabolic parameters linked to physical performance in endurance runners.

Methods: Autogenic training group $\left(\mathrm{AT}_{\mathrm{ER}}, 9\right.$ males and $\left.1 \mathrm{female}\right)$ and a matched control group $\left(\mathrm{CT}_{\mathrm{ER}}\right)$ underwent the following protocol: an incremental exercise test till exhaustion on field track with a pace increasing of $10 \mathrm{~s}$ per $\mathrm{km}$ (T0); the same test after 3 months of AT ( $\mathrm{AT}_{\mathrm{ER}}$ group only) (T1). AT protocol consisted in one individual session per day with 3 exercise and in a weekly group session lasted $90 \mathrm{~min}$. The athletes wore a GPS system and a portable gas analyzer during the field track tests. Oxygen uptake $\left(\mathrm{VO}_{2}\right)$, carbon dioxide production $\left(\mathrm{VCO}_{2}\right)$, pulmonary ventilation $(\mathrm{Ve})$ and heart rate $(\mathrm{HR})$ were measured. Oxygen pulse (OP) and the excess $\mathrm{CO}_{2}$ production $\left(\mathrm{CO}_{2 \text { excess }}\right)$ was also calculated. Changes in above mentioned parameter values and changes in the runners ability to increase their speed gradually (pacing) were evaluated comparing test $\mathrm{A}$ and $\mathrm{B}$ in both groups. Results: At $\mathrm{T} 1, \mathrm{AT}_{\mathrm{ER}}$ group showed an increase in maximum speed and in $\mathrm{CO}_{2 \text { excess }}$ values $(+115 \%, \mathrm{p}<0.05$ vs. T0).

Conclusions: An appropriate AT can improve the pacing ability during an incremental field test leading to runners to perform a brilliant end-spurt sustained by the anaerobic capacity.

\section{TP1 O}

\section{Health-related field-based fitness tests: a test battery and normative values for Italian primary schools}

\section{Galvani ${ }^{1}$, E. Tommasini ${ }^{1}$, L. Correale ${ }^{2}$, M. Vandoni $^{2}$, M. Puci ${ }^{2}$, R. Codella ${ }^{3}, F$. Togni ${ }^{4}, F$. Casolo ${ }^{1}$, C. Montomoli ${ }^{2}$}

${ }^{1}$ Università Cattolica del Sacro Cuore, Milano;

${ }^{2}$ Università degli Studi di Pavia;

${ }^{3}$ Università degli Studi di Milano;

${ }^{4}$ Università degli studi di Brescia

Purpose: Physical fitness is considered an important indicator of health. Health-related fitness qualities in children have been identified: cardiorespiratory fitness, musculoskeletal fitness, motor fitness, and body composition or body mass index (BMI) [1]. Growth charts for BMI that apply to the whole Italian population has been already studied [2]. Therefore, this study was conducted to establish normative values of fitness components from Italian children using field-based and well-standardized tests $[3,4]$ that can be easily applied in a school setting, and to create a Physical Fitness Performance Index (PFPI).

Methods: This cross-sectional study examined the physical fitness among a random and large representative sample of schoolchildren aged 6-10 years. Fitness data were collected during the 2016-2017 school year from 25 schools in Lombardy. A sample of 5106 children 
(height $131.2 \pm 10.1 \mathrm{~cm}$, weight $30.5 \pm 8.4 \mathrm{~kg}$, BMI $17.4 \pm 2.9 \mathrm{~kg} /$ $\mathrm{m}^{2}$ ) was assessed using a complete test battery (six-minute walking test_6MWT, standing broad jump_SBJ, and $4 \times 10 \mathrm{~m}$ shuttle run test_SRT). Gender and age-specific percentiles for the physical fitness tests were expressed as curves values (3rd, 10th, 25th, 50th, 75th, 90th and 97th centiles), and the PFPI was also calculated according to percentiles.

Results: Boys scored higher in all the physical fitness tests when compared to girls (M: 6MWT, $616.7 \pm 87.4 \mathrm{~m}$; SBJ, $121.6 \pm 23.8 \mathrm{~cm}$; SRT, $14.5 \pm 2.2 \mathrm{~s} ;$ F: 6MWT, $607.9 \pm 82.6 \mathrm{~m}$; SBJ, $113.7 \pm 23.6 \mathrm{~cm}$; SRT, $15.0 \pm 2.2 \mathrm{~s}$; p < 0.001). There was also a trend towards increased physical fitness levels as the age increased in both boys and girls $(\mathrm{p}<0.0001)$. Correlations between scores on individual test items were moderate to high ( $\mathrm{r}$ from .549 to .700), and all individual test item scores significantly correlated with PFPI $(\mathrm{p}<0.0001)$.

Conclusions: Primary schools are a favorable environment for implementing practices that support health and the PFPI can be used by teachers for easily rating children's health-related fitness qualities. References

1. Br J Sports Med. 2011;45(6):518-24.

2. Eur J Clin Nutr. 2002;56(2):171-80.

3. Am J Respir Crit Care Med. 2002;166(1):111-7.

4. Int J Sports Med. 2011;32(3):159-69.

\section{ADAPTED PHYSICAL ACTIVITY}

\section{AP K}

\section{Impact of Sport participation and aerobic fitness on atherosclerotic cardiovascular risk in paralympic athletes with a locomotor impairment}

\author{
M. Bernardi ${ }^{1,2}$, P.G. Curatulo $^{1}$, D. Dante ${ }^{1}$, F. $_{\text {Parisi }}{ }^{1}$, \\ R. Lanzano ${ }^{1}$, M.R. Squeo ${ }^{3}$, P.E. Adami ${ }^{3}$, A. Spataro ${ }^{3}$, \\ A. Pelliccia ${ }^{3}$
}

${ }^{I}$ Department of Physiology of Pharmacology, University of Rome "Sapienza", Rome, Italy;

${ }^{2}$ Italian Paralympic Committee, Rome, Italy;

${ }^{3}$ Institute of Sports Medicine and Science, Italian National Olympic Committee, Rome, Italy

Purpose: Atherosclerotic cardiovascular disease (ACVD), the leading cause of morbidity and mortality in individuals with a locomotor impairment (I-LI), occurs in I-LI at earlier ages than in ambulatory individuals because I-LI, particularly those with a spinal cord injury (SCI), have a higher prevalence of ACVD risk factors (RF) mainly due to their increased sedentary habit. However, since the prevalence of ACVD-RF is very high even in one Brazilian study on Paralympic athletes (PA), we assessed ACVD-RF in PA with SCI (PA-SCI) and in PA with other (different from SCI) LI (PA-OLI) competing in sports with different metabolic and technical characteristics. We tested the hypothesis that aerobic fitness (oxygen uptake peak$\mathrm{VO}_{2 \text { peak }}$ ) was inversely related to ACVD-RF.

Methods: Summer and winter male PA (62 PA-SCI and 56 PA-OLI), competing in Skill Sports-SS (36 PA), Power Sports-PS (20), Mixed Metabolism Sports-MS (42) and Endurance Sports-ES (20) were screened through anthropometric and blood pressure (BP) measurements, laboratory blood tests and graded cardiopulmonary maximal exercise test, to estimate both an ACVD-RF score and $\mathrm{VO}_{2 \text { peak }}$. The ACVD-RF score was calculated summing 1 point for each of the following RF assessed in accordance with the latest guidelines: obesity, hypertension, dyslipidemia, impaired fasting glucose and subtracting 1 point when serum HDL-C was higher than $60 \mathrm{mg} \mathrm{dl}^{-1}$. Results: Based on the ACVD-RF score, 3 ACVD-RF groups were formed. PA in group $1(\mathrm{RF} \leq 0$, age $34 \pm 8.5, \mathrm{~N}=43,23 \mathrm{PA}-\mathrm{SCI})$, had a $\mathrm{VO}_{2 \text { peak }}$ equal to $36 \pm 8.3 \mathrm{ml} \mathrm{kg}^{-1} \mathrm{~min}^{-1}$ significantly higher than in PA of group $2(\mathrm{RF}=1$, age $35 \pm 9.3, \mathrm{~N}=43,19 \mathrm{PA}-\mathrm{SCI}$; $\mathrm{VO}_{2 \text { peak }}: 31 \pm 8.9 \mathrm{ml} \mathrm{kg}{ }^{-1} \mathrm{~min}^{-1}$ ) and included $65 \%$ of PA competing in $\mathrm{ES}$ and $\mathrm{MS}$. In group $3(\mathrm{RF} \geq 2$, age $41 \pm 7.8, \mathrm{~N}=37,20$ PA-SCI), which included $44.4 \%$ of the PA competing in $\mathrm{SS}, \mathrm{VO}_{2 \text { peak }}$ $\left(24 \pm 7.2 \mathrm{ml} \mathrm{kg}^{-1} \mathrm{~min}^{-1}\right)$ was significantly lower than group 2 . $\mathrm{VO}_{2 \text { peak }}$ was significantly inversely correlated with ACDR-RF score. Conclusions: High aerobic fitness and participation in high energy expenditure sports seem to provide a protective effect on ACVD-RF in PA with a LI regardless the health condition.

\section{AP O}

Respiratory muscle training in neurodegenerative disease: the state of the art

\section{S. Rampichini, C. Doria, E. Limonta, M. Borrelli, S. Longo, G. Coratella, S. Shokhoyar, L. Galasso, A. Montaruli, E. Cè, F. Esposito}

Department of Biomedical Sciences for Health, Università degli Studi di Milano

Purpose: The increasing incidence of neurodegenerative disorders leading to respiratory function impairment has enhanced the efforts of expert scientists and clinicians in considering new respiratory muscle training (RMT) strategies to delay the decline of respiratory functions. RMT is a technique used to increase strength or endurance of the respiratory muscles. The aim of this study was to review the state of the art of different RMT protocols proposed to patients with neurodegenerative diseases and ventilatory weakness, in particular in patients with amyotrophic lateral sclerosis (ALS).

Methods: A comprehensive literature search on the electronic databases PubMed, Scopus and Web of Science for English relevant articles was performed from 2000 up to June 2018. The search terms used were "Respiratory muscle training", "inspiratory muscle training", "amyotrophic lateral sclerosis", "ALS", "neuromuscular disease", and "neurodegenerative disease".

Results: Sixteen studies were found on RMT in patients with neuromuscular diseases. Most of them dealt with multiple sclerosis, a minor part treated ALS, only one study investigated the effects of RMT in Duchenne dystrophy and one in myasthenia gravis. Trainings focused on inspiratory or expiratory muscles. The treatment period ranged from 5 weeks to 2 years. The sample population spanned from one to forty-eight patients. Respiratory function parameters were evaluated together with respiratory strength indices. Functional tests, such as 6-minute walking test and functional scales specific for each pathology, were included. In few studies, no changes were found after the treatment period. On the contrary, increase in maximal inspiratory and maximal expiratory pressure and in the maximal voluntary ventilation were often reported. 
Conclusions: Mild-to-moderate intensity respiratory exercises seem to have a beneficial impact on patients with progressive neurodegenerative diseases involving respiratory impairment.

Reference

Ferreira GD, Costab AC, Plentz RD, Coronel CC, Sbruzzi G (2016) Review. Respiratory training improved ventilatory function and respiratory muscle strength in patients with multiple sclerosis and lateral amyotrophic sclerosis: systematic review and meta-analysis. Physiotherapy 102:221-228.

\section{AP O}

\section{Training methods and analysis of races of a top level paralympic swimming athlete}

\author{
L. Puce ${ }^{I}$, L. Marinelli ${ }^{1}$, E. Pierantozzi ${ }^{1}$, L. Mori ${ }^{1}$, \\ I. Pallecchi ${ }^{2}$, M. Bonifazi ${ }^{3}$, M. Bove ${ }^{4}$, E. Franchini ${ }^{5}$, \\ C. Trompetto ${ }^{1}$
}

${ }^{1}$ Department of Neuroscience, Rehabilitation, Ophthalmology, Genetics, Maternal and Child Health University of Genoa, Genoa, Italy;

${ }^{2}$ CNR-SPIN, Physics Department University of Genoa, Genoa, Italy; ${ }^{3}$ Department of Physiology, University of Siena, Siena, Italy; ${ }^{4}$ Department of Experimental Medicine, Section of Human Physiology, University of Genoa, 16132 Genoa, Italy; ${ }^{5}$ Sport Department, University of Sao Paulo, Brazil; Australian Institute of Sport, Australia

Purpose: Training methods for Paralympic swimmers must take into account different pathologies, competitions classes, athlete individual circumstances and peculiar physical adaptation mechanisms, hence general guidelines cannot be found in literature. In this study, we present a training program, implemented for the physical preparation of a top level Paralympic swimmer, affected by cerebral palsy. By the end of 2016, the athlete was holder of Italian, European, world and Paralympic titles in the $400 \mathrm{~m}$ freestyle competition, S6 class.

Method: The training macrocycle was structured in a three-fold periodization (three mesocycles), in view of the preparation to three international competitions. The four-month training mesocycles prior to each competition differed substantially in terms of mileage load, intensity and recovery times. The first mesocycle was characterized by a sizeable low-intensity mileage load, the second one was shifted to lower mileage load, carried out at middle-to-high intensity levels, the third one entailed increased effort intensity, counterbalanced by lower mileage load. In all cases, recovery times were balanced to obtain optimized performance through physical adaptation to training stimuli, keeping into account the physiopathological response of the athlete. Tapering phases were adjusted to maximize the performance at the competitions.

Results: As an assessment of the effectiveness of the training method, correspondence between chronometric and technical parameters in the three competitions and the respective mesocycle training programs was found

Conclusions: The results of the present study may support the development of training guidelines for athletes affected by upper motor neuron lesions.

Reference

Puce L et al (2018) J Exerc Rehabil (in press)

\section{AP O}

Perceived barriers to physical activity among parents

E. Gobbi ${ }^{1}$, I. Ferri ${ }^{1}$, A. Carraro ${ }^{1}$

${ }^{1}$ Department of Biomedical Sciences, University of Padua, Italy

Purpose: Youth with intellectual disabilities (ID) exhibit low levels of physical activity (PA) and one of the underlying contributors to this behaviour is represented by the family, that plays a key role in facilitating or hampering the participation of their offsprings with ID in PA (Alesi and Pepi 2017). The purpose of this study was to investigate parental perceived barriers to PA among youth with ID who play sport.

Methods: Sixty-one parents $(67.2 \%$ mothers, mean age $53.4 \pm 6.8$ years) of youth with mild to severe ID ( $37.7 \%$ girls, mean age $20.7 \pm 6.1$ years) enrolled in a sport activity answered a questionnaire package investigating variables referred to their son/daughter (Greguol et al. 2015). Selected variables were perceived barriers to PA, independence in the activity of daily living (ADL), PA and sedentary behaviours.

Results: The most reported parental perceived barriers to their offspring' PA were the "attitude of others regarding the disability" (45\%), "few programs available for PA and sport" (42.4\%), and "lack of information about available PA programs" (39.7\%). Parental perceived barriers did not differ by youth gender or age, level of ID, place where they live, nor the youth independence in ADL. A significant negative correlation were found between weekly parentreported youth PA and barriers perception $(r=-.285, p<.05)$.

Conclusions: Findings suggest that, even if all the youth with ID were involved in a sport activity, their parents perceived significant barriers to PA. Particularly, negative attitudes and prejudice of other people could hamper PA participation of youth with ID. Moreover, more information should be provided on sport and PA opportunities for youth with ID. Future studies should explore parental perceived barriers among parents of youth who do not play a sport, or in an international comparison.

References

Alesi M, Pepi A (2017) Physical activity engagement in young people with Down syndrome: Investigating parental beliefs. J Appl Res Intellect Disabil 30(1):71-83

Greguol M, Gobbi E, Carraro A (2015) Physical activity practice among children and adolescents with visual impairment-influence of parental support and perceived barriers. Disabil Rehabil 37(4):327330 


\section{AP O}

\section{Wind of change: benefits of water sports in people with multiple sclerosis}

\section{Bonzano ${ }^{1}$, L. Pedullà ${ }^{2,3}$, P. Canepa $^{3}$, A. Tacchino $^{2}$, C. Monteleoni ${ }^{3}$, M.A. Battaglia ${ }^{4}$, G. Brichetto ${ }^{2}$, M. Bove $^{3}$}

${ }^{1}$ Department of Neuroscience, Rehabilitation, Ophthalmology, Genetics, Maternal and Child Health, University of Genoa, Genoa, Italy;

${ }^{2}$ Italian Multiple Sclerosis Foundation, Scientific Research Area, Genoa, Italy;

${ }^{3}$ Department of Experimental Medicine, Section of Human

Physiology, University of Genoa, Genoa, Italy;

${ }^{4}$ Department of Life Science, University of Siena, Siena, Italy

Purpose: People with multiple sclerosis (PwMS) are less physically active than non-diseased peers, especially due to perceived fatigue and low self-efficacy preventing them from experiencing a large number of physical activities [1]. However, physical activity could positively influence their motor and cognitive domains and even induce neuroprotection [2]. Here we investigated the impact of the practice of water sports (sailing, windsurfing and stand-up paddling), commonly believed too challenging by PwMS.

Methods: To reduce barriers in facing up to physical activities and evaluate the effects of a 5-day water sports training on upper limb, balance and walking performances, PwMS with minimal disability were asked to participate in the event "Wind of Change" in Isola d'Elba, promoted by the Italian Multiple Sclerosis Foundation and the University of Genoa in May 2018. Descriptive measures were collected by means of validated patient-reported outcomes for fatigue (Modified Fatigue Impact Scale-MFIS), walking (12-item MS Walking Scale-MSWS-12) and upper limb (Arm Function in MS Questionnaire-AMSQ) functions. Instrumental tests to assess upper limb (9-Hole Peg Test-9HPT, grip and pinch), balance and walking (25-foot walk test-25FWT at self-paced and maximal speed, 2-minute walk test-2MWT, Timed Up and Go-TUG) abilities were administered before and after the training.

Results: Seventeen relapsing-remitting PwMS (14 females) were recruited from different parts of Italy; none of them previously experienced the proposed water sports. No minor events, relapses, and/or neurological worsening were reported in the last 3 months. Mean age was $29.5 \pm 3.5$ years, MFIS $19.28 \pm 14.68$, MSWS-12 $16.71 \pm 6.04$, AMSQ $33.94 \pm 7.43$. Participants were able to practice all the proposed activities, with positive attitude notwithstanding their subjective fatigue in daily living typical of the disease.

They reported improved self-efficacy due to the proposed water activities and showed significant improvement in right 9HPT (pre $16.9 \mathrm{~s}$, post $15.9 \mathrm{~s}, \mathrm{p}<0.01$ ), left $9 \mathrm{HPT}$ (pre $17.9 \mathrm{~s}$, post 16.6 , $\mathrm{p}<0.005$ ), 25FWT self-paced (pre $5.32 \mathrm{~s}$, post $4.61 \mathrm{~s}, \mathrm{p}<0.005$ ), 25FWT fast (pre $3.81 \mathrm{~s}$, post $3.63 \mathrm{~s}, \mathrm{p}<0.02$ ), 2MWT (pre $175.01 \mathrm{~m}$, post $192.44 \mathrm{~m}, \mathrm{p}<0.01$ ), TUG (pre $6.46 \mathrm{~s}$, post 6.15 , $\mathrm{p}=0.03$ ). No significant improvement was found in grip and pinch. Conclusions: All these findings suggested that a 5-day water sports program could have a positive effect on upper limb, balance, walking and perceived self-efficacy in PwMS.

Acknowledgements: This study was supported by the Italian Multiple Sclerosis Foundation.

References

1. Casey B, Uszynski M, Hayes S, Motl R, Gallagher S, Coote S (2018) Do multiple sclerosis symptoms moderate the relationship between self-efficacy and physical activity in people with multiple sclerosis? Rehabil Psychol 63(1):104
2. Prakash RS, Snook EM, Motl RW, Kramer AF (2010) Aerobic fitness is associated with gray matter volume and white matter integrity in multiple sclerosis. Brain Res 1341:41-51

\section{AP 0 \\ Adapted physical activity and retraction of Achilles tendons due to perinatal hypoxia: a case report study}

\author{
S. Ottobrini ${ }^{1}$, I. Bui ${ }^{1}$, F. Gentile ${ }^{I}$, A. Gaudio ${ }^{1}$, \\ M. Del Bianco ${ }^{1}$, L. Marin ${ }^{1,2}$
}

${ }^{1}$ Laboratory of Adapted Motor Activity (LAMA), Department of Public Health, Experimental Medicine and Forensic Science, University of Pavia, Pavia, Italy;

${ }^{2}$ Technology for Sports Medicine and Rehabilitation, Department of Industrial Engineering, University of Rome "Tor Vergata", Rome, Italy

Purpose: Perinatal hypoxia can have various consequences on multiple apparatuses, including the muscle-tendon, which may undergo retraction. Adapted physical activity (APA) can be a valid tool for acting on the problems of the musculoskeletal system, alleviating the impact they have on the activities of daily living. The aim of this case report study was to evaluate the effects of an APA program on the flexibility and walking ability of an adult subject, born preterm and with retraction of both Achilles tendons resulting from hypoxia at birth.

Methods: The subject of the study was a 40-year-old woman, normalweight, born preterm, with retraction of the Achilles tendons due to perinatal hypoxia. The subject's flexibility and walking ability were evaluated at the beginning $\left(\mathrm{T}_{0}\right)$ and at the end $\left(\mathrm{T}_{1}\right)$ of a 4 weeks APA program. The subject performed these evaluations: Sit and Reach, Time Up and Go, Timed 10-Meter Walk test, baropodometric analysis. The training program, characterized by two sessions per week (one supervised and one homebased), was aimed to improving both the flexibility of retracted structures and walking abilities. Each work session consisted of analytical and global stretching exercises and strengthening exercises.

Results: At the end of the program, the subject improved the performance both in the flexibility (Sit and Reach T0: $-19 \mathrm{~cm}$; T1: $-15 \mathrm{~cm}$ ) and in the walking abilities (Time Up and Go $\mathrm{T}_{0}: 10 \mathrm{~s}, \mathrm{~T}_{1}$ : $7.86 \mathrm{~s}$; Timed 10-Meter Walk Test $\mathrm{T}_{0}: 5.50 \mathrm{~s}, \mathrm{~T}_{1}: 3.94 \mathrm{~s}$ ). No modifications were recorded in the baropodometric analysis.

Conclusions: The results of this study suggest that APA program could be a good mean to improve the flexibility of the posterior kinetic chain and the performances related to the walking abilities in subjects with retraction of the Achilles tendons due to perinatal hypoxia. There were no variations in the distribution of load on the plantar surface, probably because it is difficult to modify these parameters in a short time period. The prosecution of the training program could lead to benefits also in this field.

\section{References}

Bouiller JP, Dreyfus M, Mortamet G, Guillois B, Benoist G. (2015), Intrapartum asphyxia: Risk factors and short-term consequences. Journal de gynécologie obstétrique et biologie de la reproduction 45(6):626-632.

Ruzbarsky JJ, Scher D, Dodwell E. (2016) Toe walking: causes, epidemiology, assessment, and treatment. Current opinion in pediatrics 28(1):40-46. 


\section{SATURDAY ORAL SESSION I}

\section{EXERCISE PHYSIOLOGY 1}

\section{EP1 K \\ Physiological determinants of mechanical efficiency during advanced aging and skeletal muscle disuse}

M. Venturelli ${ }^{1}$, G. R. Morgan ${ }^{2,3}$, E. Muti ${ }^{4}$, C. Tarperi $^{1}$,

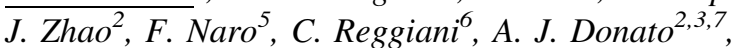
R. S. Richardson ${ }^{2,3,7}$, F. Schena ${ }^{1}$

${ }^{1}$ Department of Neurosciences, Biomedicine and Movement Sciences. University of Verona, Italy;

${ }^{2}$ Division of Geriatrics, Department of Internal Medicine, University of Utah School of Medicine, University of Utah, Salt Lake City, Utah, USA;

${ }^{3}$ Geriatric Research, Education, and Clinical Center, George E. Whalen Department of Veterans Affairs Medical Center, Salt Lake City, Utah, USA;

${ }^{4}$ Mons Mazzali Foundation, Mantova, Italy;

${ }^{5}$ DAHFMO Unit of Histology and Medical Embryology, Sapienza

University, Rome, Italy;

${ }^{6}$ Department of Biomedical Sciences, University of Padua, Padua, Italy;

${ }^{7}$ Department of Exercise and Sport Science, University of Utah, Salt Lake City, Utah, USA;

Purpose: The aim of this study was to determine which physiological factors impact mechanical efficiency in oldest-old individuals at different stages of skeletal muscle disuse.

Methods: To this aim, we examined mechanical efficiency, central hemodynamics, peripheral circulation, and peripheral factors (skeletal muscle fiber type, capillarization Ncaf, and concentration of mitochondrial DNA [mtDNA]). Twelve young (YG; $25 \pm 2$ years) and 12 oldest-old mobile (OM; $87 \pm 3$ years) and 12 immobile (OI; $88 \pm 4$ years) subjects performed knee extensor (KE) and elbow flexor (EF) exercises. Pulmonary oxygen uptake, photoplethysmography and Doppler ultrasound were used to assess mechanical efficiency, central and peripheral hemodynamics. We also collected muscle biopsies of the vastus lateralis and biceps brachii and measured skeletal muscle fiber type, Ncaf, and [mtDNA] to assess peripheral adaptations to advanced aging and disuse.

Results: Mechanical efficiency of lower-limb muscle of the OM was $15 \%$ greater than $\mathrm{YG}(\mathrm{P}=0.03)$, while OI subjects demonstrated $24 \%$ lower mechanical efficiency during $\mathrm{KE}$ than $\mathrm{YG}(\mathrm{P}=0.02)$. These differences in mechanical efficiency during KE were coupled with significant peripheral adaptations, revealing very good correlations between proportion of type I fiber $(\mathrm{r}=0.91, \mathrm{P}=0.01)$, and [mtDNA] $(\mathrm{r}=0.89, \mathrm{P}=0.04)$. No differences were found in upper-limb muscle mechanical efficiency between YG, OM, and OI subjects.

Conclusions: These findings suggest that the mechanical efficiency is reduced by muscle inactivity and not by chronological age, per se. Likewise, this study revealed that age-related changes in mechanical efficiency are not a consequence of central or peripheral hemodynamic adaptations, but are likely a product of peripheral changes related to the skeletal muscle fiber type and mitochondrial content

\section{EP1 O}

The effects of pedalling cadence on respiratory frequency: passive vs. active exercise

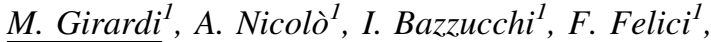 \\ M. Sacchet $i^{1}$
}

${ }^{1}$ Department of Movement, Human and Health Sciences, University of Rome "Foro Italico", Piazza Lauro De Bosis 6, Rome 00135, Italy

Purpose: Emerging evidence suggests the existence of a differential control between respiratory frequency $\left(f_{\mathrm{R}}\right)$ and tidal volume [1]. While $f_{\mathrm{R}}$ appears to be mainly regulated by the magnitude of central command during exercise, pedalling cadence (via muscle afferent feedback and entrainment) is often considered as an important factor affecting $f_{\mathrm{R}}$. While this is true in animals, ${ }^{[2]}$ controversial findings have been found in humans. Therefore, we aimed to investigate the effects of pedalling cadence on $f_{\mathrm{R}}$ in humans.

Methods: Ten well-trained male cyclists performed a preliminary ramp incremental test and three experimental visits on separate days. The experimental protocol consisted of sinusoidal variations in pedalling cadence between 55 and $115 \mathrm{rpm}$ (sinusoidal period of 4-min) during passive exercise (PE, the participant's legs were moved passively via an instrumented tandem ergometer), moderate exercise (ME, 50\% of the first ventilatory threshold) and heavy exercise (HE, $80 \%$ of the peak power output). A frequency analysis was used to obtain the amplitude (A) and phase lag $(\varphi)$ of cardiorespiratory and electromyographic variables.

Results: During PE, $f_{\mathrm{R}}$ changes in accordance with variations in pedalling cadence $\left(\mathrm{A}=3.9 \pm 1.4\right.$ breaths $\min ^{-1} ; \varphi=-5.3 \pm 13.9$ degrees). Conversely, the effect of pedalling cadence on $f_{\mathrm{R}}$ is reduced during $\mathrm{ME} \quad\left(\mathrm{A}=2.6 \pm 1.3\right.$ breaths $\min ^{-1} ; \quad \varphi=-25.4 \pm 26.3$ degrees $)$ and is even more reduced during $\mathrm{HE}(\mathrm{A}=1.8 \pm 1.0$ breaths $\cdot \min ^{-1} ; \varphi=70.1 \pm 44.5$ degrees). No entrainment was found between pedaling cadence and $f_{\mathrm{R}}$ in any of the exercise conditions tested.

Conclusion: During PE, the effect of pedalling cadence on $f_{\mathrm{R}}$ seems to be mediated by mechanical muscle afferent feedback, but not via entrainment. However, this effect is moderated by the magnitude of central command, and it is, therefore, very low during HE.

\section{References}

Nicolò A, Girardi M, Sacchetti M (2017) Control of the depth and rate of breathing: metabolic vs. non-metabolic inputs. J Physiol 595(19):6363-6364

Potts JT, Rybak IA, Paton JF (2005) Respiratory rhythm entrainment by somatic afferent stimulation. J Neurosci 25(8):1965-1978

\section{EP1 O}

\section{A new model of ventilatory control during exercise}

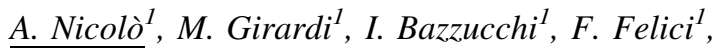
M. Sacchetti ${ }^{1}$

${ }^{I}$ Department of Movement, Human and Health Sciences, University of Rome "Foro Italico", Piazza Lauro De Bosis 6, Rome 00135, Italy

Purpose: Differentiating between respiratory frequency $\left(f_{\mathrm{R}}\right)$ and tidal volume $\left(\mathrm{V}_{\mathrm{T}}\right)$ may provide further insight into the control of ventilation during exercise because $f_{\mathrm{R}}$ and $\mathrm{V}_{\mathrm{T}}$ seem to be regulated by different inputs (Nicolò et al. 2017a, b). We proposed a series of 
exercise manipulations to improve our understanding of how $f_{\mathrm{R}}$ and $\mathrm{V}_{\mathrm{T}}$ are regulated during exercise.

Methods: Twelve cyclists performed an incremental test and three randomized experimental sessions in separate visits. In two of the three experimental visits, participants performed a moderate-intensity sinusoidal test followed, after recovery, by a moderate-to-severe-intensity sinusoidal test. These two visits differed in the sinusoidal period ( $2 \mathrm{~min}$ vs. $8 \mathrm{~min}$ ). In the third experimental visit, participants performed a trapezoidal test where the work rate was self-paced in order to match a predefined trapezoidal template of rating of perceived exertion (RPE)

Results: The results collectively reveal that $f_{\mathrm{R}}$ changes more with RPE than with work rate, $\dot{\mathrm{V}} \mathrm{CO}_{2}, \dot{\mathrm{V}} \mathrm{O}_{2}, \mathrm{~V}_{\mathrm{T}}$ or the amount of muscle activation. However, $f_{\mathrm{R}}$ dissociates from RPE during moderate exercise. Both $\mathrm{V}_{\mathrm{T}}$ and minute ventilation $\left(\dot{\mathrm{V}}_{\mathrm{E}}\right)$ showed a similar time course and a strong correlation with $\mathrm{VCO}_{2}$ in all the tests. Nevertheless, $\dot{\mathrm{V} C O}{ }_{2}$ was associated more with $\dot{\mathrm{V}}_{\mathrm{E}}$ than with $\mathrm{V}_{\mathrm{T}}$ because $\mathrm{V}_{\mathrm{T}}$ seems to adjust continuously on the basis of $f_{\mathrm{R}}$ levels to match $\dot{\mathrm{V}}_{\mathrm{E}}$ with $\dot{\mathrm{V}} \mathrm{CO}_{2}$.

Conclusions: The present findings provide novel insight into the differential control of $f_{\mathrm{R}}$ and $\mathrm{V}_{\mathrm{T}}$-and their unbalanced interdependence-during exercise. The emerging model of ventilatory control is expected to guide future research aiming to unravel the mechanisms underlying the long-debated issue of exercise hyperpnoea.

\section{References}

Nicolò A, Girardi M, Sacchetti M (2017a) Control of the depth and rate of breathing: metabolic vs. non-metabolic inputs. J Physiol 595:6363-6364

Nicolò A, Marcora SM, Bazzucchi I, Sacchetti M (2017b) Differential control of respiratory frequency and tidal volume during highintensity interval training. Exp Physiol 102:934-949

\section{EP1 O}

\section{Decreasing intervals HIIT increase time above $90 \%$ $\mathrm{V}^{\prime} \mathbf{O}_{2} \mathbf{m a x}$}

\author{
F. Vaccari $^{l}$, N. Giovanelli ${ }^{1}$, S. Lazzer $^{1}$ \\ ${ }^{1}$ Department of Medicine, University of Udine, Italy
}

Purpose: Maximal aerobic power $\left(\mathrm{V}^{\prime} \mathrm{O}_{2} \max \right)$ could be improved working at minimal power eliciting $\mathrm{V}^{\prime} \mathrm{O}_{2} \max$ (Midgley 2006). High Intensity Interval Training (HIIT) is one of the best way to improve $\mathrm{V}^{\prime} \mathrm{O}_{2}$ max. HIIT is defined as either repeated short $(<45 \mathrm{~s})$ to long $(2-$ $4 \mathrm{~min}$ ) bouts of rather highbut not maximal-intensity exercise. While short HIIT bouts could improve time limit $\left(\mathrm{T}_{\mathrm{Lim}}\right)$ (Turner et al. 2006), long intervals could reach quickly $\mathrm{V}^{\prime} \mathrm{O}_{2} \max$ (Millet 2003),

Then, the aim of the present study was to evaluate if time above $90 \%$ $\mathrm{V}^{\prime} \mathrm{O}_{2}$ max increase pooling together long intervals followed by short intervals.

Methods: 13 amateur cyclists (age $42 \pm 10$ years) participated in this study. $\mathrm{V}^{\prime} \mathrm{O}_{2} \max (57 \pm 5 \mathrm{ml} / \mathrm{min} \mathrm{kg})$ and Critical Power (CP) $(249 \pm 33 \mathrm{~W})$ were measured. Then subjects performed randomly three HIIT tests until exhaustion. Every protocols had same intensity $(\sim 115 \% \mathrm{CP})$ and same ratio Work/relief. One Long Interval HIIT $\left(\mathrm{LI}_{\text {HUT }}\right.$ ) (3 min High-, $2^{\prime}$ Low-intensity), one Short Intervals HIIT ( $\left.\mathrm{SI}_{\mathrm{HIT}}\right)$ (30 s High-, $20^{\prime \prime}$ Low-intensity) and finally decreasing intervals HIIT ( $\mathrm{DI}_{\mathrm{HITT}}$ ) (combining High intensity from $3^{\prime}$ to $30^{\prime \prime}$ and low intensity from $2^{\prime}$ to $\left.20^{\prime \prime}\right)$. $\mathrm{T}_{\text {Lim }}$, Time above $90 \% \mathrm{~V}^{\prime} \mathrm{O}_{2} \max$, lactate concentration [la] (at rest, 3rd min and at exhaustion) and perceived exertion (PE) (at 3rd $\min$ and at exhaustion) were measured.
Results: $\mathrm{T}_{\mathrm{Lim}}$ were similar in $\mathrm{DI}_{\mathrm{HITT}}$ and $\mathrm{SI}_{\mathrm{HIIT}}(793 \mathrm{~s} \pm 178$ and $737 \pm 267 \mathrm{~s})$ but lower $(\mathrm{p}<0.05)$ in $\mathrm{LI}_{\mathrm{HIIT}}(667 \pm 270 \mathrm{~s})$. Time above $90 \% \mathrm{~V}^{\prime} \mathrm{O}_{2}$ max was greater $(\mathrm{p}<0.05)$ in $\mathrm{DI}_{\mathrm{HIIT}}(288 \pm 217 \mathrm{~s})$ than $\mathrm{SI}_{\mathrm{HIT}}(168 \pm 221 \mathrm{~s})$ and $\mathrm{LI}_{\mathrm{HITT}}(165 \pm 147 \mathrm{~s})$, at $3^{\text {th }}$ min [la] were similar in $\mathrm{DI}_{\mathrm{HITT}}$ and $\mathrm{LI}_{\mathrm{HIT}}(7.81 \pm 2.69 \mathrm{mmol} / \mathrm{L}$ and $7.77 \pm 2.88 \mathrm{mmol} / \mathrm{L})$ but lower $(\mathrm{p}<0.05)$ in $\mathrm{SI}_{\mathrm{HIIT}}$ $(5.56 \pm 1.62 \mathrm{mmol} / \mathrm{L})$. At exhaustion, no differences in [la] and PE were found between protocols.

Conclusion: $\mathrm{DI}_{\mathrm{HIT}}$ showed higher Time above $90 \% \mathrm{~V}^{\prime} \mathrm{O}_{2} \max$ than $\mathrm{LI}_{\mathrm{HIIT}}$ and $\mathrm{SI}_{\mathrm{HIIT}}$ which suggest higher efficiency in activating aerobic metabolism.

\section{References}

Midgley A, McNaughton L (2006) Time at or near VO2max during continuous and intermittent running. J Sports Med Phys Fitness 46:114

Millet GP et al (2003) Responses to different intermittent runs at velocity associated with. Can J Appl Physiol 28(3):410-423

Turner AP et al (2006) Oxygen uptake and muscle desaturation kinetics during intermittent cycling. Med Sci Sports Exerc 38(3):492503

Keywords: $\mathrm{HIIT}, \mathrm{V}^{\prime} \mathrm{O}_{2} \max ; \mathrm{V}^{\prime} \mathrm{O}_{2} \max$ training; Time at $\mathrm{V}^{\prime} \mathrm{O}_{2} \max$

\section{EP1 O}

\section{Comparison of physiological response "boundaries" during dynamic incremental two-legged knee-extension vs leg cycling exercise}

\author{
E. Faelli ${ }^{1}$, S. Pogliaghi ${ }^{1}$, L.K. Love ${ }^{2,3}$, M.D. Hodgson ${ }^{2,3}$, \\ J.M. Kowalchuk ${ }^{2,3,4}$
}

${ }^{I}$ Department of Neuroscience, Biomedicine and Movement Science, University of Verona, Verona, Italy;

${ }^{2}$ Canadian Centre for Activity and Aging;

${ }^{3}$ School of Kinesiology;

${ }^{4}$ Department of Physiology and Pharmacology, The University of Western Ontario, London, Ontario, Canada

Purpose: The dynamic knee-extension (KE) exercise model is used to study metabolism of the knee-extensors and understand the physiological mechanisms that affect it. During KE-exercise, similar to leg cycling (C), the quadriceps muscle activity increases in proportion to power output; however, the minimisation of movement of the thigh and upper body allows invasive measures that would be impossible during cycling [1]. While the single-leg KE exercise model has been described [1], a more physiologically relevant two-legged KE model has received less characterisation. To describe and compare the physiological responses to incremental two-legged $\mathrm{KE}$ and $\mathrm{C}$ exercise to the limit of tolerance.

Methods: Ten healthy males ( $26 \pm 5$ years) performed in random order an incremental cycling $\left(\mathrm{C}, 20 \mathrm{~W}\right.$ baseline $+25 \mathrm{~W}$ min $^{-1}$ increment) and $\mathrm{KE}$ exercise (KE, $3 \mathrm{~W}$ baseline $+3 \mathrm{~W} \mathrm{m^{-1 }}$ increment) to exhaustion. Power output (PO) and heart rate (HR) were recorded; $\mathrm{O}_{2}$ uptake $\left(\mathrm{VO}_{2}\right)$, ventilation (VE), respiratory exchange ratio (RER) were determined breath-by-breath. Peak values and the submaximal "boundaries" gas exchange threshold (GET) and respiratory compensation point (RCP) were determined for both exercises and compared by Student's paired $t$ test.

Results: Subjects reached higher peak values with C vs KE (PO, $378 \pm 72 \quad$ vs $\quad 87 \pm 15 \mathrm{~W} ; \quad \mathrm{VO}_{2}, \quad 3.99 \pm 0.87 \quad$ vs $1.84 \pm 0.28 \mathrm{~L} \mathrm{~min}^{-1}$; VE $194 \pm 18$ vs $1245 \pm 27 \mathrm{~L} \mathrm{~min}^{-1}$, HR $184 \pm 9$ vs $152 \pm 19 \mathrm{~b} \mathrm{~min}^{-1} p<0.001$ for all comparisons). On the contrary, RER was not different in $\mathrm{C}(1.4 \pm 0.1)$ vs $\mathrm{KE}(1.1 \pm 0.2$ 
$p=0.12)$. GET and RCP were detected at significantly higher $\mathrm{VO}_{2}$ in $\mathrm{C}$ vs $\mathrm{KE}\left(2.30 \pm 0.53\right.$ vs $1.03 \pm 0.26 \mathrm{~L} \mathrm{~min}^{-1} p<0.001$ and $3.11 \pm 0.62$ vs $1.42 \pm 0.18 \mathrm{~L} \mathrm{~min}^{-1} p<0.001$ for GET and RCP respectively), yet they represented a similar fraction of the exercisespecific $\mathrm{VO}_{2 \text { peak }}$ in both exercise modes $(58 \pm 5$ vs $56 \pm 106 \%$ $p=0.52$ and $78 \pm 4$ vs $78 \pm 9 \%, p=0.65$ for GET and RCP respectively).

Conclusions: Incremental two-legged KE exercise, that engages $30 \%$ of the muscle mass and is characterised by a $\sim 3$-fold higher perfusion-to-muscle mass ratio compared to cycling [2], is associated with a peak $\mathrm{VO}_{2}$ that is $\sim 50 \%$ of that of $\mathrm{C}$ and a maximal $\mathrm{HR}$ and VE response that are respectively 80 and $60 \%$ of that of C. Exercise intensity "boundaries", as measured by GET and RCP, represent a similar fraction of the exercise-specific peak $\mathrm{VO}_{2}$ in both exercise modes.

References

1. Andersen P, Adams R P, Sjogaard G, Thorboe A, Saltin B (1985) J Appl Physiol 59:1647-1653

2. Poole DC, Richardson RS (1997) Sports Med 24:308-320

\section{TRAINING, PERFORMANCE AND EVA- LUATION METHODS 2}

\section{TP2 K \\ Sleep hygiene (SH) improves sleep latency but not sleep quality in response to an acute evening session of small- sided games (SSG) in soccer players}

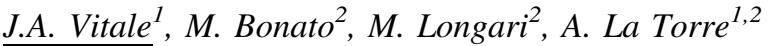 \\ ${ }^{1}$ IRCCS Istituto Ortopedico Galeazzi, Milano, Italy; \\ ${ }^{2}$ Department of Biomedical Sciences for Health, University of Milano, Milano, Italy}

Purpose: Sleep plays an essential role athletes' well-being and recovery (Bonnar et al. 2018). Several endogenous and exogenous factors are able to negatively influence sleep in athletes and both intensity and timing of physical activity seem to play a key role too (Vitale et al. 2017). In order to optimize elite athletes 'sleep, it has been suggested to improve their $\mathrm{SH}$. We aimed to evaluate the effect of a sleep behavioral strategy on actigraphy-based sleep parameters in soccer players.

Methods: Twenty-nine male soccer players (age $25.5 \pm 4.6$; BMI $22.8 \pm 1.1$ ), all classified as neither-types, wore an actigraph (Actiwatch 2, Philips) for a week to detect their sleep and were randomized into two groups: intervention (INT; $\mathrm{N}=17$ ) or control (CON; $N=12$ ). All players performed a high-intensity SSG session at 20:00: after training, INT followed a $\mathrm{SH}$ while $\mathrm{CON}$ did not. We studied their Sleep Efficiency (SE, \%), Immobile Time (IT, \%), Sleep Latency (SL, min) and the Fragmentation Index (FI, \%) both the three nights before (PRE) and the two nights after the SSG session (POST 1 and POST 2).

Results: The Mixed-ANOVA procedures highlighted no differences between groups in SE, IT, and FI at PRE, POST 1, and POST 2. On the contrary, SL showed significant variations: INT decreased SL from PRE to POST 1 by $48 \%$ ( $p<0.01$; from 9.6 to $5.0 \mathrm{~min}$ ) while $\mathrm{CON}$ showed only a trend toward the increase of this parameter.
Conclusions: SH resulted in a decrease in the time required for falling asleep but did not affect other sleep parameters in soccer players. Implementation of behavioral strategies and sleep hygiene techniques in athletes are needed.

References

Bonnar D, Bartel K, Kakoschke N, Lang C (2018) Sleep interventions designed to improve athletic performance and recovery: a systematic review of current approaches. Sports Med 48(3):683-703

Vitale JA, Bonato M, Galasso L, La Torre A, Merati G, Montaruli A, Roveda E, Carandente (2017) Sleep quality and high intensity interval training at two different times of day: a crossover study on the influence of the chronotype in male collegiate soccer players. Chronobiol Int 34:260-268

\section{TP2 O}

Body composition, physical performance and functional movement patterns in youth male soccer players: which are the best predictors for repeated-sprint ability?

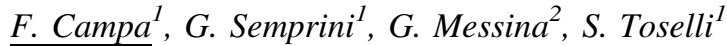

${ }^{1}$ Department of Biomedical and Neuromotor Sciences, University of Bologna, Bologna, Italy;

${ }^{2}$ School of Pharmacy, Biotechnology and Motor Sciences, University of Bologna, Bologna, Italy

Purpose: This study aimed to establish which factors among anthropometrics features, neuromuscular factors, physical performance measures and functional movement patterns (FMP) were the best predictors for repeated-sprint ability (RSA).

Methods: Thirty-six youth male soccer players (aged $16.61 \pm 0.49$ years, BMI $21.99 \pm 1.28 \mathrm{~kg} / \mathrm{m}^{2}$ ) participated in the study. On separate days, the athletes completed the RSA test and other physical tests including countermovement jump (CMJ), $20 \mathrm{~m}$ straightline sprint, Yo-Yo Intermittent Recovery Test Level 1 (Yo-Yo), and functional movement screen (FMS). RSA performance components such as best time, mean team and sprint decrement were calculated.

Results: Measures of muscular power derived from a horizontal plane in $20 \mathrm{~m}$ sprints, were more strongly associated $(\mathrm{p}<0.001)$ with RSA performance than to the other obtained with CMJ $(\mathrm{p}<0.05)$. Significant correlations $(p<0.001)$ were also found between RSA components and Yo-Yo distance, as well as with FMS. Fat mass, upper fat area, calf muscle and fat area, thigh fat area and endomorphy showed a moderate correlation with RSA components $(\mathrm{p}<0.05)$. Predictors of the RSA performance in the stepwise multivariate analysis included Yo-Yo distance, time in sprint, FMP and calf muscle area. On the contrary, the explosive power measure evaluated with $\mathrm{CMJ}$ was not included into the predictive model of RSA performance. Conclusions: The most related factors to RSA performance in this study were aerobic fitness level measured by Yo-Yo test, speed over $10 \mathrm{~m}$ and FMP. Moreover, a low body fat can help youth soccer players to achieve optimal RSA performances.

\section{References}

Brocherie F, Girard O, Forchino FA, Haddad H, Dos Santos GA, Millet GP (2014) Relationships between anthropometric measures and athletic performance, with special reference to repeated-sprint ability, in the Qatar national soccer team. J Sports Sci 32(13):12431254

Castagna C, Manzi V, Impellizzeri F, Weston M, Barbero Alvarez JC (2010) Relationship between endurance field tests and match performance in young soccer players. J Strength Cond Res 24(12):3227-3233 


\section{TP2 O Relative age effect in soccer: a study on Italian clubs competing in Serie A and relative youth sectors}

\author{
P.R.Brustio ${ }^{1}$, C. Lupo $^{1}$, A.N. Ungureanu ${ }^{1}$, R. Frati $^{2}$, \\ $\overline{\text { A. Rainoldi }^{l}}$, G. Boccia ${ }^{1}$ \\ ${ }^{I}$ NeuroMuscularFunction Research Group, School of Exercise \\ and Sport Sciences, Department of Medical Sciences, University \\ of Turin, Turin, Italy; \\ ${ }^{2}$ School of Exercise and Sport Sciences, SUISM, University of Torino, \\ Turin, Italy
}

Purpose: Asymmetry in birth-date distribution (i.e., Relative Age Effect, RAE) is a bias observed in sport competitions, especially in young categories. This bias, due to early physical and cognitive maturation of older athletes (i.e., athletes born close to the data of selection), may benefit sport performance and consequently the process of sport talent identification. The aim of this study was to investigate the RAE in Italian soccer clubs competing in Serie A and the relative youth sectors.

Methods: Birth-dates of soccer players competing in the Italian 2017-2018 championship (categories: Under 15, U15; Under 16, U16; Under, U17; Primavera; Serie A) were collected. Quartiles of birth-date distributions (i.e., January-March, Q1; April-June, Q2; July-September, Q3; October-December, Q4) and the week of birth distributions were considered for the analysis.

Results: Considering the overall sample, the first and second quartile were over-represented (mean $=70.7 \%$ ) compared to the third and fourth quartile (mean $=29.3 \%$ ). The observed distributions for U15, U16, U17, Primavera, and Serie A were significantly different from the uniform distribution ( $\mathrm{p}$ values $<0.001$ ). Generally, effect size for each category decreased as age increases, but remains large across all youth categories (ranged 0.47-0.22). Poisson regressions showed that the players born right at the start of the data of selection were approximately 9, 12, 9, 9 and 3 times more likely to be included in U15, U16, U17, Primavera and Serie A respectively compared to those born at the end of the data of selection ( $\mathrm{p}$ values $<0.001$ ).

Conclusions: Data suggested a trend of an over-representation of soccer players born close to the data of selection for both youth sector and Serie A clubs. Athletes born close to the data of selection might have more chances to be selected in both young and senior category. However, RAE decreased after the adolescence period. During sport talent identification coaches and scouts should consider RAE, to select the promising players in the long-term rather than the best performers at the time of selection.

\section{TP2 O \\ Evaluation of the coordinative and technical skills in young football players by means of video analysis}

\author{
L. Zaccagni, N. Guerzoni, M. Tessarin, L. Ercolin
}

Department of Biomedical and Specialty Surgical Sciences, University of Ferrara, Italy

Purpose: There are different formative stages in sportive development leading to different training plans. The aim of the present study was to identify differences in coordinative skills and technical skills of young football players through a new quantitative method of assessment.

Methods: The study was carried out on a sample of 30 children of the category "pulcini" of the Spal Academy in Ferrara. They have played football for an average of $4.0 \pm 1.6$ years and they trained $5 \mathrm{~h}$ per week. We tested these young players using a mobile laboratory consisting of two suitably positioned cameras: one frontal and the other in side position, each one on a tripod at a height of $0.80 \mathrm{~m}$. Each child was videotaped during the execution of five forward and reverse coordinative gaits (walking, run, march, skip and soldier). Among technical skills we encoded "kick the ball". Each coordinative and technical skill included 3-4 performance criteria: if the child performed the component correctly, the examiner marked 1 , conversely he marked 0 . At the end of the evaluation process, a coordination score and a technical score were achieved.

Results: The young players obtained an average coordination score of $14.2 \pm 3.1$ (equal to $37 \%$ of the maximum possible value) and an average technical score of $9.3 \pm 4.1$ (equal to $47 \%$ of the maximum possible value). The technical score was positively correlated to coordinative score $(r=0.542 ; p=0.02)$. Based on the years of football practice, the players were divided into two groups: "beginners", with a practice of less than 4 years, and "experts", with a practice of more than 4 years. Only coordinative score was significantly lower in the "beginners" group.

Conclusion: We proposed a method to quantitatively evaluate the coordinative level and technical skill of young football players by means of video analysis. It is a simple, field and coach-friendly method. The application of our method on a sample of young football players highlighted that school-age children, followed by appropriate training plans, improved their coordinative skills learning the technical skills specific of their sport.

Reference

Legnani G, Palmieri G, Fassi I (2018) Introduzione alla biomeccanica dello sport. Edizioni Città Studi, Torino 


\section{PHYSICAL EXERCISE AS PREVENTION AND THERAPY 1}

\section{EH1 K \\ Evaluating the effect of daily physical exercise on endometriosis symptoms}

\author{
V. Cavedon ${ }^{1}$, S. Garzon ${ }^{2}$, M.A. Carrozza ${ }^{3}$, P. Pomini ${ }^{2}$, \\ C. Zancanaro ${ }^{1}$, C. Milanese ${ }^{I}$
}

\begin{abstract}
${ }^{I}$ Department of Neurological, Biomedical and Movement Sciences, University of Verona, Verona, Italy;

${ }^{2}$ Department of Medicine, University of Verona, Verona, Italy; ${ }^{3}$ Private practice in nutrition, Verona, Italy; Obstetrics and Gynecology Department at the Hospital University of Verona
\end{abstract}

Purpose: Endometriosis is one of the most common gynaecological disorders among reproductive-aged women characterized by a range of pelvic pain symptoms, dysmenorrhea, dysuria, dyschezia and dyspareunia [1]. Today there is a lack of objective information regarding the physical activity levels and their association with pain in this population. The aim of this study is to cross-sectionally investigate the association between objectively-measured daily physical activity (DPA) and the endometriosis symptoms.

Methods: 23 women in reproductive age (age $39.48 \pm 4.81$ years and Body Mass Index $24.75 \pm 5.24 \mathrm{~kg} / \mathrm{cm}^{2}$ ) with clinically diagnosed endometriosis volunteered in this study. DPA was monitored using the SenseWear Armband over 7 consecutive days monitoring and endometriosis-associated symptoms were quantified on a visual analogue scale. The association between the considered variables was calculated by the Spearman's rank correlation coefficient $(\rho)$.

Results: The footsteps/days and the daily duration of physical activity were negatively correlated with dysmenorrhea $(\rho=-0.569$, $\mathrm{P}=0.005$ and $\rho=-0.577, \mathrm{P}=0.004$, respectively), chronic pelvic pain $(\rho=-0.591, P=0.003$ and $\rho=-0.696, P \leq 0.001$, respectively) and dyspareunia $(\rho=-0.671, P \leq 0.001$ and $\rho=-0.446$, $\mathrm{P}=0.033$, respectively). The sedentary time as well as the time spent on light-intensity (i.e. $<3$ metabolic equivalents $[\mathrm{MET}$ ] $]$ ) and vigorous-intensity (i.e. $>6$ METs) physical activity were not significantly correlated with the endometriosis-related symptoms. Instead, a significant negative association was observed between the time spent per day in moderate-intensity physical activity (i.e. 3-6 METs) and dysmenorrhea $(\rho=-0.686, P \leq 0.001)$, chronic pelvic pain $(\rho=-0.691, \quad P \leq 0.001), \quad$ dyspareunia $(\rho=-0.584$, $\mathrm{P}=0.003)$ and dysuria $(\rho=-0.584, \mathrm{P}=0.014)$. The results suggested a potential benefit of physical activity in the reduction of the endometriosis-related symptoms. As active lifestyle is modifiable, this may be an important area for future interventions in this population. Intervention studies are therefore needed to confirm and expand these findings.

Reference

1. Ricci E et al (2016) Physical activity and endometriosis risk in women with infertility or pain: systematic review and meta-analysis. Medicine (Baltimore) 95(40):e4957 (Review)

\section{EH1 O}

\section{Structured pathway of exercise prescription program for patients with type 2 diabetes and its effects on insulin therapy}

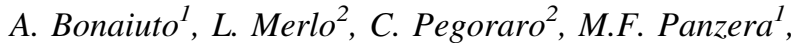 \\ R. Restuccia ${ }^{1}$, L. Magaudda ${ }^{1}$, P. Sarto ${ }^{2}$
}

${ }^{1}$ Department of Biomedical, Dental Sciences and Morphological and Functional Images, Postgraduate School of Sports Medicine, University of Messina, Messina, Italy;

${ }^{2}$ U.O.C. di Medicina dello sport e dell'Esercizio Fisico, Aulss 2 Marca trevigiana, Treviso, Italy

Purpose: Type 2 Diabetes Mellitus is a chronic disease which needs a complex therapeutic approach. Guidelines recommend physical activity as a non-pharmacological therapeutic strategy for treatment and control of type 2 diabetes. Aim of this communication is to describe the program in place at the Sports Medicine and exercise prescription Unit in Treviso, Italy, and its effects on the insulin therapy, if compared to a group of diabetics following home based therapy.

Methods: The program stems from a collaboration between sports medicine (SM) physicians, general practitioners (GP) and diabetologists. GP and diabetologists refer their patients to the sports medicine unit for a medical evaluation to establish patients' eligibility for the program and the goals for each patient. After the preliminary evaluation, patients undergo further medical examination if deemed necessary and a stress test or cardiopulmonary exercise test to identify target training heart rate and workload and screen for cardiovascular disease. The third step is the supervised exercise training in the SM gym where training intensity is adjusted according to medical needs and patient's tolerance. Patients are instructed to adjust insulin dosage to avoid hypoglycemia and to exercise safely with respect to exercise timing versus meals and insulin administration, foot care etcetera. Once the patients reach the established goals, they are discharged from the SM gym and receive a medical report for their GP and diabetologist and a written exercise prescription. The Sports Medicine Unit in Treviso has a collaboration with a network of private gyms and health clubs in the Treviso area, where patients can continue to exercise safely under the supervision of qualified trainers. Patients who continue to exercise undergo a periodical follow-up evaluation with updating of the exercise prescription. Also, we compared two groups of diabetics, selected by their own choice of continuing to follow the program in local gyms or by their own, to evaluate differences in insulin therapy administration.

Results and conclusions: We've found that, during the period between enrollment and discharge from health facility, both groups showed a significant reduction of insulin therapy administered (about $-30 \%$ and $20 \%$ of respectively fast-acting and long-acting insulin); successively this outcome was maintained by both groups, but with higher significance level in diabetics undergoing the supervised program in local gyms, allowing us to deduce that following this pathway is effective in the reduction of the amount of insulin therapy needed, maintaining a good glicemic control and this outcome over time ( 6 months follow up). Reference

Colberg SR, Sigal RJ, Yardley JE, Riddell MC, Dunstan DW, Dempsey PC, Horton ES, Castorino K, Tate DF (2016) Physical activity/exercise and diabetes: a position statement of the American Diabetes Association. Diabetes Care 39(11):2065-2079

Standards of Medical Care in Diabetes (2018) Diabetes Care 41(Suppl 1) American College of Sports Medicine. ACSM's guidelines for exercise testing and prescription, 10th edn. Wolters Kluwer Keywords: Exercise Prescription, Diabetes, Programme 


\section{EH1 O}

\section{The effect of exercise program, lifestyle plan without caloric restriction for 12 months on woman affected by Diabetes type 1}

\section{E. D'Angelo $^{1,2}$, M. Amicone ${ }^{1,2}$, M. Di Finizio $^{1,2}$, M.G. Vinciguerra ${ }^{2}$}

${ }^{1}$ Training Lab Italia, Independent Research Laboratory, Pescara, Italy;

${ }^{2}$ Scienze Motorie DISCAB, Università degli Studi L'Aquila, Coppito, Italy

Purpose: The prevalence of Diabetes is growing worldwide and exercise in patients has many benefit. Hypoglycemia is most often discussed and usually treated with an exercise plan and drugs. Sedentary lifestyle promotes high blood sugar whereas high physical activity (PA) level prevent its accumulation. Aim of the study was to investigate the patient who successfully managed his type 1 diabetes with a tailored exercise plan, lifestyle modification without caloric restriction.

Methods: A 25-year-old nurse with a body mass index of $27 \mathrm{~kg} / \mathrm{m}^{2}$ was examined within a 1 year exercise plan at Training Lab Italia, monitoring body composition, hyperglycemia and lifestyle modification. The amount of the physical activity was assessed with IPAQ and the intensity of the exercise was determined with Borg Scale. The exercise plan consisted, according to International Guidelines of Diabetes treatment, $150 \mathrm{~min} /$ week or $1000 \mathrm{MET} /$ week but the single session include a Circuit Training Lab Italia Protocol (CTLIP); eight station with $30 \mathrm{~s}$ of exercise and $30 \mathrm{~s}$ of rest each exercise 3 time/ session 3 day/week.

Results: Data showed the positive glycemic control post exercise $(\mathrm{p}<0.05)$, on body composition and the amount of the daily physical activity $(\mathrm{p}<0.05)$. his patient with severe diabetic complications participated safely and successfully in an exercise program. Problems with glycemic control occurred at the start of the study but did not preclude adherence to incremental exercise.

Conclusion: Our experience in this case and review of the literature, emphasize the importance of continue practice of aerobic and strength conditioning whole an high level of physical activity seems to be, without a caloric restriction, the key to improve health status.

References

Wormgoor SG et al (2018) Acute blood glucose, cardiovascular and exaggerated responses to HIIT and moderate-intensity continuous training in men with type 2 diabetes mellitus. J Sports Med Physiol Fitness.

Yang $\mathrm{Z}$ et al (2014) Resistance exercise versus aerobic exercise for type 2 diabetes: a systematic review and meta-analysis. Sports Med

\section{EH1 O}

Six months of Nordic Walking and Walking activity

in obese middle aged population: the best way for an improvement in physical fitness and body composition

\author{
V. Muollo ${ }^{1,2}$, C. Milanese ${ }^{1}$, A. Rossi $^{3}$, E. Masciocchi ${ }^{3}$, \\ M. Taylor ${ }^{3}$, F. Schena ${ }^{1,2}$
}

${ }^{I}$ Department of Neurosciences, Biomedicine, and Movement Sciences, University of Verona;

${ }^{2}$ CeRiSM, Sport Mountain and Health Research Centre, University of Verona, Rovereto; ${ }^{3}$ Section of Geriatrics, Department of Medicine, University of Verona

Purpose: Nordic Walking (NW) has been recently proposed as a specific physical activity in preventing and counteracting the negative changes related to obesity [1]. However no long longitudinal studies [2] have evaluated this whole body exercise to the reference and simplest form of locomotion i.e. Walking (W). The aim of this study was to compare the effects of a long supervised period of NW vs W activities on the aerobic and functional capacities, on upper and lower limbs' strength, on the anthropometric and body composition indices in an obese middle-aged population.

Methods: 27 subjects were divided in NW $(n=15,66 \pm 7$ years, BMI $33 \pm 5)$ and $\mathrm{W}(\mathrm{n}=12,66 \pm 8$ years, BMI $32 \pm 5)$ groups. They performed supervised training 3 times-week for 24 weeks; during each session peak heart rate $\left(\mathrm{HR}_{\text {peak }}\right)$ and rating of perceived exertion (RPE) were recorded. The following tests at baseline, after 3 and 6 months were conducted: (a) aerobic capacity $\left(\mathrm{VO}_{2 \text { peak }}\right.$, Peak Power Output (PPO)); (b) functional capacity (six minute walking test (6MWT), arm curl (AC), chair stand (CS)); (c) strength (maximal voluntary contraction of brachial biceps $\left(\mathrm{MVC}_{\mathrm{BB}}\right)$ and femoral biceps $\left.\left(\mathrm{MVC}_{\mathrm{FB}}\right)\right)$; (d) BMI and body composition (total/compartmental fat and lean mass).

Results: During the training sessions $\mathrm{HR}_{\text {peak }}$ was significantly higher in NW than in W group with a significantly lower RPE in NW. In 6 months PPO increased in NW and in W (12\% vs $10 \%$ respectively, $\mathrm{p}<0.001)$ with an improvement in $\mathrm{VO}_{2 \text { peak }}$ in $\mathrm{NW}(8 \%, \mathrm{p}<0.05)$. After 3 and 6 months, NW improved significantly in 6 MWT (7\% and $13 \%, \mathrm{p}<0.001), \mathrm{AC}(20 \%$ and $35 \%, \mathrm{p}<0.05)$ and CS $(21 \%$ and $29 \%, \mathrm{p}<0.05$ ) compared to W. A significant increase over time was observed in $\mathrm{NW}$ in $\mathrm{MVC}_{\mathrm{BB}}$ and $\mathrm{MVC}_{\mathrm{FB}}$. Both groups had similar reductions in $\mathrm{BMI}(\mathrm{NW}: 6 \%, \mathrm{p}<0.01 ; \mathrm{W}: 4 \%, \mathrm{p}<0.05)$ and $\mathrm{WB}_{\text {tot }}$ (NW: $8 \%, \mathrm{p}<0.001 ; \mathrm{W}: 7 \%, \mathrm{p}<0.01$ ), but NW decreased trunk fat $(6 \%, \mathrm{p}<0.05)$ and had a greater reduction in leg fat $(9 \%, \mathrm{p}<0.01)$ than W. No statistical differences were observed in lean mass.

Conclusions: Our results suggested that NW has a specific capacity to produce, at least in some parameters relevant for health, greater and faster benefits than $\mathrm{W}$; no negative reports were linked to the utilization of this more complex form of locomotion. These data confirm that NW has a specific advantage for obese subjects and sustain NW diffusion as a primary tool to reduce the impact of obesity in middleaged person.

References

Figard et al (2010) Eur J Appl Physiol

Bullo et al (2017) Rejuvenation Res 


\section{PHYSICAL EDUCATION AND SPORT PEDAGOGY 1}

\section{EH1 O \\ Relationship between individual ventilatory threshold and maximal fat oxidation in different obese classes}

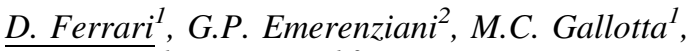 \\ L. Guidetti ${ }^{l}$, C.Baldari ${ }^{1,3}$
}

${ }^{1}$ Department of Movement, Human and Health Sciences, University of Rome "Foro Italico", Rome, Italy; ${ }^{2}$ Department of Experimental and Clinical Medicine, University Magna Graecia, Catanzaro, Italy; ${ }^{3}$ eCampus University, Novedrate (CO), Italy

Purpose: To evaluate the relationship between Individual Ventilatory Threshold (IVT) and Maximal Fat Oxidation (MFO) in women with class I, II and III obesity.

Methods: Fifty-two female obese adults (age $=4 \pm 11$ years; BMI $=$ $38.5 \pm 5.2 \mathrm{~kg} \mathrm{~m}^{-2}$ ) were involved in this study. Subjects were divided into three groups: Obese Class I (OBI, $30<\mathrm{BMI}<34.9 \mathrm{~kg} \mathrm{~m}^{-2}$, $\mathrm{n}=16$ ); Obese Class II (OBII, $35<$ BMI $<39.9 \mathrm{~kg} \mathrm{~m}^{-2}, \mathrm{n}=20$ ), Obese Class III (OBIII, BMI $>40 \mathrm{~kg} \mathrm{~m}^{-2}, \mathrm{n}=16$ ). After body composition assessment, all subjects performed an incremental graded exercise test in order to evaluate peak oxygen uptake $\left(\mathrm{VO}_{2 \text { peak }}\right)$, IVT and MFO. MFO was evaluated using a stoichiometric equation. Fat max zone was determined for each subject within $10 \%$ of fat oxidation rates at MFO.

Results: Weight, BMI, percent of fat mass, were significant different between classes. $\mathrm{VO}_{2 \text { peak }}$ and MET were significantly higher in OBI than other two classes. Heart rate (HR), percent of HR maximum $\left(\% \mathrm{HR}_{\max }\right)(70.0 \pm 8.8$ vs $69.0 \pm 9.9 \%$ at IVT and MFO, respectively), oxygen uptake $\left(\mathrm{VO}_{2}\right)$, percent of $\mathrm{VO}_{2 \text { peak }}\left(\% \mathrm{VO}_{2 \text { peak }}\right)(73.0 \pm$ 11.0 vs $70.2 \pm 12.7 \%$ at IVT and MFO, respectively), and gait speed (GS) $\left(4.5 \pm 0.8\right.$ vs $4.3 \pm 0.9 \mathrm{~km} \mathrm{~h}^{-1}$ at IVT and MFO, respectively), were statistically significant different $(\mathrm{P} \leq 0.05)$ between IVT and MFO. Significant exercise $\times$ obesity classes differences were found for $\mathrm{VO}_{2}$ and $\% \mathrm{VO}_{2}$. Post hoc analysis showed that $\mathrm{VO}_{2}$ and $\% \mathrm{VO}_{2 \text { peak }}$ at IVT were significantly higher than MFO in OBII. Moreover, $\% \mathrm{VO}_{2 \text { peak }}$ at MFO was significantly higher in OBIII than other two classes. MFO rates were significantly higher in OBIII $\left(655.0 \mathrm{mg} \cdot \mathrm{min}^{-1}\right)$ than other two classes (497.6 and $532.9 \mathrm{mg} \mathrm{min}^{-1}$ in OBI and OBII respectively). In all subjects, IVT was within the fat max zone.

Conclusions: Even if there were statistically differences between exercise intensity corresponding to IVT and MFO, these differences are not relevant for practical application. Moreover, IVT was within the fat max zone, indicating that the exercise intensity corresponding to IVT may be used to prescribe physical activity that maximize fat oxidation.

\section{References}

Meyer T, Lucía A, Earnest CP, Kindermann W (2005) A conceptual framework for performance diagnosis and training prescription from submaximal gas exchange parameters-theory and application. Int $\mathbf{J}$ Sports Med 1:S38-48

Emerenziani GP, Gallotta MC, Migliaccio S, Ferrari D, Greco EA, Saavedra FJ, Iazzoni S, Aversa A, Donini LM, Lenzi A, Baldari C, Guidetti L (2018) Effects of an individualized home-based unsupervised aerobic training on body composition and physiological parameters in obese adults are independent of gender. J Endocrinol Invest 41:465-473
Venables MC, Jeukendrup AE (2008) Endurance training and obesity: effect on substrate metabolism and insulin sensitivity. Med Sci Sports Exerc 40:495-502

Tan S, Wang J, Cao L, Guo Z, Wang Y (2014) Positive effect of exercise training at maximal fat oxidation intensity on body composition and lipid metabolism in overweight middle-aged women. Clin Physiol Funct Imaging 36:225-30

\section{PE1 K \\ Self-efficacy and enjoyment in children: factorial validation of two pictorial scales}

\section{Bortoli, C. Robazza}

BIND-Behavioral Imaging and Neural Dynamics Center, Department of Medicine and Aging Sciences, "G. d'Annunzio" University of Chieti-Pescara, Italy

Purpose: To examine the factor structure of two scales measuring self-efficacy and enjoyment levels among 6to 7-year-old children in the context of primary school physical education. Self-efficacy and enjoyment are two main constructs proposed within many motivational theories in any human endeavor (see Ryan and Deci 2017), sport and physical activity included.

Methods: The sample consisted of 14,035 6to 7-year-old children (6960 girls and 7075 boys) assessed in small groups during physical education lessons. The self-efficacy scale was comprised of four items assessing perceived speed, strength, coordination, and fatigue. Scores of each item could range from 1 to 4 using verbal anchors (e.g., from "I run very slow" to "I run very fast"), emoticons, and pictograms. The enjoyment scale was also comprised of four items (e.g., "I enjoy myself") anchored by emoticons. Scores could range from 1 (not at all) to 5 (very, very much). Children evaluated themselves on each item thinking about their physical education experience.

Results: The factorial validity of the scales was examined using confirmatory factor analysis (CFA) with Mplus 8.1. Single factor CFA models were estimated using the maximum likelihood parameter estimates with standard errors and a mean-adjusted Chi square test statistic robust to non-normality. CFA yielded satisfactory fit indices on both measures: self-efficacy, CFI $=.998, \mathrm{TLI}=.993$, RMSEA $=$ $.021(90 \% \mathrm{CI}=.012-.032),. \mathrm{SRMR}=.007$; enjoyment, $\mathrm{CFI}=.971$, $\mathrm{TLI}=.912, \mathrm{RMSEA}=.044(90 \% \mathrm{CI}=.035-.055), \mathrm{SRMR}=.022$ (all standardized factor scores $>.500$ ). Reliability alpha values (Cronbach) were .666 for the self-efficacy scale and .722 for the enjoyment scale. The correlation between the latent constructs of the two scales was .442 .

Conclusions: The results supported the single factor structure of the scales, which can be easily administered to 6to 7-year-old children to assess relevant psychological constructs in physical education. Additional studies are recommended to examine, in particular, the concurrent and predictive validity of the scales.

\section{Reference}

Ryan RM, Deci EL (2017) Self-determination theory: basic psychological needs in motivation, development, and wellness. The Guilford Press, New York 


\section{PE1 O} The Finnish School on the Move programme: policy-
making and implementation strategies for Italy

\section{A. Borgogni}

Department of Human Sciences, Society, and Health, University of Cassino and Southern Lazio

Purpose: To analyse from scientific and policy viewpoints the Finnish Schools on the Move program inferring strategies and actions applicable to the Italian case.

Methods: The presentation introduces, through a fact sheet, the actual Finnish situation about physical activity policies and research. The analysis of the updated scientific literature is complemented by the description of the program's background and current design.

Beyond the literature review, qualitative methods were applied: a documentary analysis, interviews $(\mathrm{n}=2)$ with key informants, and field observations $(n=3)$ of schools participating in the program.

Results: Presuming that there are not comprehensive models transferrable from a country to another and referring to a conceptual model of children's active behaviours, the article emphasizes, for the Italian case, the feasible actions: at short term, changing rules at school level, those achievable at medium term, changing organization at community level, and those requiring deeper cultural and legislative modifications.

At policy making level, it is plausible that some programs and actions of the Finnish program would be costless in Italy: some depend on a better destination of already available resources, several are possible through inter-sectors policies; all the strategies and actions, however, should require to be assessed and monitored through researches entrusted to a university network in collaboration with sport and public health institutions and organizations.

\section{References}

Aira A, Kämpi K (2017) Towards more active and pleasant school days. Interim report on the Finnish Schools on the Move programme 2015-2016. Jyväskylä: Kirjapaino Kari

Borgogni A, Arduini M, Digennaro S (in print) Mobilità attiva, autonomia e processi educativi nell'infanzia e nell'adolescenza. MeTis

Borgogni A (2018) The Finnish School on the Move programme: policy-making and implementation strategies for Italy. Eur J Res Educ Teaching XVI-1:171-181

Tammelin TH, Aira A, Hakamäki M, Husu P, Kallio J, Kokko S, Laine K, Lehtonen K, Mononen K, Palomäki S, Ståhl T, Sääkslahti A, Tynjälä J, Kämppi K (2016) Finland's 2016 Report Card on physical activity for children and youth. J Phys Activity Health 13(11 Suppl 2):S157-S164

Keywords: School on the Move program, physical activity, policymaking, implementation

\section{PE1 O}

The role of context factors in sport motivation: a comparison between disabled athletes and nondisabled athletes

\section{A. M. Murdaca, P. Oliva}

Department of Cognitive Sciences, Psychology, Educational and Cultural Studies, University of Messina

Purpose: According to the Self-Determination Theory, the satisfaction of basic psychological needs (autonomy, competence and relatedness) and the parental and coach support, in athletes, play a crucial role in the sports motivation. However, studies investigating the role of social contexts in determining behavior regulation for physical exercise are still insufficient. The aim of the present study was to analyze the role of context factors (parental support and coach support) in the satisfaction of psychological basic needs and sports motivation in disabled and non-disabled adolescents engaged in competitive sports activities.

Methods: Forty competitive athletes (age $\mathrm{M}=14.98$; $\mathrm{SD}=2.130$ ), of which 20 athletes with disabilities and 20 athletes without any disabilities. Each subject was asked to complete the following questionnaires: Psychological Basic Need Satisfaction; Sport Climate Questionnaire; Behavioral Regulation Exercise Questionnaire; Perception of Parent Scale.

Results: Results showed that non-disabled athletes are perceived as more autonomous $[p>.05]$ and competent $[p>.05]$ compared to peers with disabilities. Regarding the contexts, athletes with disabilities seem to perceive a greater involvement and maternal warmth $[p>.05]$ and greater support from the coach $[p>.05]$ compared to peers without disabilities. With regard to sport motivation, both groups show high levels of self-regulation exercise, confirming what is claimed in the literature about the tendency to assume highly selfdetermined attitudes and supported by motivations and intrinsic interests; and this seems to be valid regardless of the physical and psychological limitations of the athlete.

Conclusions: Further analysis, still underway, will allow more specific identification of the role that parents and coaches have on the satisfaction of basic needs and on sport motivation level.

\section{Reference}

Owen KB, Smith J, Lubans DR, Ng JYY, Lonsdale C (2014) Selfdetermined motivation and physical activity in children and adolescents: a systematic review and meta-analysis. Prevent Med 67:270279

Rocchi M, Pelletier LG, Couture AL (2013) Determinants of coach motivation and autonomy supportive coaching behaviours. Psychol Sport Exerc 14:852-859

Ryan RM, Deci EL (2002) An overview of self-determination theory. In: Deci EL, Ryan RM (eds) Handbook of self-determination research. University of Rochester Press, Rochester, pp 3-33

Soenens B, Vansteenkiste M (2010) A theoretical upgrade of the concept of parental psychological control: proposing new insights on the basis of self-determination theory. Dev Rev 30:74-99 


\section{PE1 O}

\section{Walking, running and playing barefoot on the beach}

\author{
M.C. Gallotta ${ }^{1}$, C. Verdini ${ }^{1}$, G.P. Emerenziani ${ }^{2}$, \\ L. Guidetti ${ }^{1}$, C. Baldari ${ }^{1,3}$ \\ ${ }^{1}$ Department of Human, Movement and Health Sciences, University \\ of Rome "Foro Italico", Italy; \\ ${ }^{2}$ Department of Experimental and Clinical Medicine, University \\ Magna Graecia, Catanzaro, Italy; \\ ${ }^{3}$ eCampus University, Novedrate (CO), Italy
}

Purpose: The first aim of this study was to compare the effectiveness of two different physical education programs conducted by a specialist physical education teacher on children's fitness and health. The second aim of this study was to evaluate the effectiveness of a physical education intervention conducted in a natural environment (the beach) on children's skilland health-related outcomes.

Methods: The study involved 106 primary school children (710 years of age) of the second, third and fifth grade: 42 children were randomly assigned to the control group and 64 children to the experimental group. The intervention period lasted 4 months and consisted of two 1-h sessions per week. Preand post-intervention tests assessed children's anthropometric parameters (weight, height, body fat percentage, BMI, abdominal circumference), health parameters (systolic and diastolic blood pressure, resting heart rate), fitness parameter $\left(\mathrm{VO}_{2 \text { peak }}\right)$, gross motor coordination and physical activity level.

Results: Both groups significantly improved fitness and motor coordination $(\mathrm{p}<0.001)$ but worsened some anthropometric parameters (weight, abdominal circumference) $(\mathrm{p}<0.001)$ after the intervention period. No differential changes on coordinative development were observed between the two groups. No significant results were observed for blood pressure and physical activity level.

Conclusions: Results of this study demonstrated that children benefitted from a well-structured intervention conducted and supervised by a specialist physical education teacher improving their motor skills and fitness. The study offered an effective alternative way to the curricular physical activity program at school.

\section{References}

Gobbi E, Elliot C, Varnier M, Carraro A (2016) Psychometric properties of the physical activity questionnaire for older children in Italy: testing the validity among a general and clinical pediatric population. PLoS One 11:e0156354

Kiphard EJ, Schilling F (1974) Körperkoordinationstest für Kinder. Beltz Test GmbH, Weinheim

Kiphard EJ, Schilling F (2007) Körperkoordinationstest für Kinder. Beltz Test GmbH, Weinheim

Lohman TG, Roche AF, Martorell R (1988) Anthropometric standardization reference manual. Human Kinetics Books, Champaign

Matsuzaka A, Takahashi Y, Yamazoe M, Kumakura N, Ikeda A, Wilk B, Bar-Or O (2004) Validity of the multistage 20-m shuttle-run test for Japanese children, adolescents, and adults. Pediatr Exerc Sci $16: 113-125$

The Cooper Institute (2006) Fitnessgramm/activitygram test administration manual. Human Kinetics, Champaign

\section{EXERCISE PHYSIOLOGY 2}

\section{EP2 K \\ Effects of isolated muscle training on central and peripheral vasomotor response and blood flow}

\author{
E. Cè, A.V. Bisconti, S. Longo, S. Rampichini, E. Limonta, \\ G. Coratella, S. Shokohyar, C. Doria, L. Galasso, \\ E. Roveda, F. Esposito
}

Department of Biomedical Sciences for Health, Università degli Studi di Milano, Italy

Purpose: The vasomotor response, a marker of cardiovascular health, refers to the adaptability of the inner lining of blood vessels to maintain a homeostasis of vascular tone. The two main control systems that underpin this response are $[1,2]$ : the systemic autonomic control, with the sympathetic branch mediated vasoconstriction, and the endothelial cells response to mechanical and chemical stimuli, that results in smooth muscle cell vasodilation. During exercise the peripheral circulation is mostly mediated by local control, and the peripheral blood flow (BF) increases in all body districts directly involved as well as those not involved in the exercise. The vasodilation extent depends on the magnitude of the stimulus received. To date, it is unknown whether vascular adaptations in the brachial artery occur as a result of isolated leg muscle training. Repetitive changes in $\mathrm{BF}$ occurring during exercise training may act as vascular training for vessels supplying the active muscle bed as wells as vessels distal to the active muscle.

To evaluate the effects of isolated quadriceps muscle training (IQT) on vasomotor response in the lower limb directly involved with exercise (femoral artery) and on the upper limb, not involved with IQT (brachial artery).

Methods: Ten healthy participants (age $23 \pm 3$ years, height $170 \pm 11 \mathrm{~cm}$, body mass $66 \pm 11 \mathrm{~kg}$, BMI $23 \pm 1 \mathrm{~kg} \mathrm{~m}^{-2}$ ) underwent 8 weeks of IQT at different intensities. Maximum work rate (MWR) was assessed before, during (every 2 weeks, to redefine the training work-load) and after IQT. Preand post-IQT measurements of vascular function and peripheral $\mathrm{BF}$ were performed by flow-mediated dilatation (\%FMD) in the brachial artery (non-trained limb), and by passive limb movement (PLM) in femoral artery (trained limb). Significance is set at $P<0.05$.

Results: After 8 weeks of IQT, MWR and BF in the femoral artery increased significantly by $43 \%(P=0.002)$ and $153 \%(P<0.001)$, respectively. No difference in $\%$ FMD was found despite the $21 \%$ increase in brachial artery blood flow during exercise.

Conclusions: Eight weeks of IQT improved peripheral vasomotor response only in the lower limb directly involved in IQT without affecting vascular function in the uninvolved upper limb, suggesting that IQT did not provide a sufficient stimulus to induce vasculature adaptations in other districts.

\section{References}

Celermajer DS, Sorensen KE, Gooch VM, Spiegelhalter DJ, Miller OI, Sullivan ID, Lloyd JK, Deanfield JE (1992) Non-invasive detection of endothelial dysfunction in children and adults at risk of atherosclerosis. Lancet 340(8828):1111-1115

Anderson TJ, Uehata A, Gerhard MD, Meredith IT, Knab S, Delagrange D, Lieberman EH, Ganz P, Creager MA, Yeung AC (1995) Close relation of endothelial function in the human coronary and peripheral circulations. J Am Coll Cardiol 26:1235-1241 


\section{EP2 O}

\section{Effects of short-term strength training on muscle fibre} conduction velocity

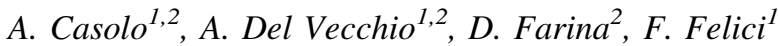 \\ ${ }^{1}$ University of Rome "Foro Italico", Rome, Italy; \\ ${ }^{2}$ Imperial College, London, UK
}

Purpose: Muscle fiber conduction velocity (MFCV) represents the propagation speed of the action potentials along the muscle fibers [1]. MFCV has received little attention in sport sciences field as part of the force production biological structure [2]. This study explored neuromuscular adaptations to a short-term strength training program with a focus on MFCV, a predictor of the progressive recruitment of motor units [1].

Methods: Sixteen healthy males completed either isometric ankle dorsi-flexion strength training (INT: $\mathrm{n}=8$; age, $25.1 \pm 2.3$ years; BMI, $23.7 \pm 2.4 \mathrm{~kg} \mathrm{~m}^{-2}$ ) or maintained their habitual activities (CON: $\mathrm{n}=8$; age, $26.1 \pm 3.9$ years; BMI, $23.3 \pm 1.9 \mathrm{~kg} \mathrm{~m}^{-2}$ ) for 4 weeks. Training involved 40 unilateral explosive contractions and 30 gradually increasing contractions up to $75 \%$ of maximal voluntary force (MVF) with a $3 \mathrm{~s}$ plateau. Testing sessions included recordings of muscle force and myoelectric activity of tibialis anterior muscle with high-density surface EMG, during maximal and linearly increasing ramp contractions. MFCV was estimated non-invasively from multichannel surface EMG signals [3]

Results: At the baseline both groups showed very similar MVF (INT $295.2 \pm 59.5 \mathrm{~N} ; \mathrm{CON} 306.6 \pm 39.1 \mathrm{~N} ; \mathrm{P}>0.05)$, as well as average MFCV. After the intervention, MVF increased significantly only in the INT group (Pre 295.2 $\pm 59.5 \mathrm{~N}$; Post $338.2 \pm 45.8 \mathrm{~N}$; $16.9 \pm 15.8 \% ; \mathrm{P}<0.001)$. Similarly, the average MFCV during submaximal ramp contractions increased significantly only in the INT group (Pre $3.89 \pm 0.5 \mathrm{~m} \mathrm{~s}^{-1}$; Post $4.23 \pm 0.5 \mathrm{~m} \cdot \mathrm{s}^{-1} ; 9.0 \pm 4.5 \%$; $\mathrm{P}<0.001)$.

Conclusions: Considering the close association between MFCV and muscle fibres diameter, the increase in MFCV following a systematic strength training regimen might indicate a larger recruitment of high threshold motor units [4]. A faster MFCV could also reflect modifications at the $\mathrm{Na}^{+} / \mathrm{K}^{+}$-ATPase pump level, which regulates the excitability of the sarcolemma [2]. An increase of average motor unit firing rate might also be responsible of this improvement [5].

\section{References}

Del Vecchio (2017) J Appl Physiol 123:835-843

Del Vecchio (2017) J Appl Physiol 123:835-843

Farina D (2001) Med Biol Eng Comput 39:225-236

Andreassen S, Arendt-Nielsen L (1987) J Physiol 391:561-571

Masuda T (1991) J Electromyogr Kinesiol 1:116-23

\section{EP2 O \\ Blood flow regulation during exercise: the role of central command}

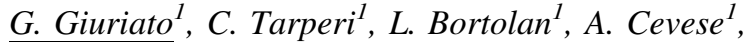 \\ F. Schena ${ }^{1}, M$. Venturelli ${ }^{1,2}$
}

${ }^{1}$ Department of Neurosciences, Biomedicine and Movement Sciences, University of Verona, Italy;

${ }^{2}$ Department of Internal Medicine, University of Utah, USA

Purpose: The control of peripheral circulation during exercise is a complicated and multifaceted phenomenon involving neural (central command and sympathetic nerve activity) and peripheral aspects (nitric oxide, temperature and $\mathrm{pH}$ ). Despite the involvement of central command in the regulation of central hemodynamics at the onset and during steady-state exercise is already known, its contribution on peripheral hemodynamics is not fully elucidated. Therefore, the purpose of the present study was to measure the peripheral hemodynamics during electrically-evoked muscle contractions (without central command) in comparison to voluntary muscle contractions (with central command).

Methods: Peripheral (femoral blood flow) and central hemodynamics were collected in 10 young healthy men at rest and during repeated quadriceps electrical stimulations (ES) $(1 \mathrm{~Hz} / 5 \mathrm{~min})$ coupled with voluntary muscle contractions (VC). Force output, total work activity and oxygen uptake were equal for both ES and VC.

Results: At the onset of exercise, both ES and VC, exhibited an equal hyperemia $(787.2 \pm 193 \mathrm{ml} / \mathrm{min}$ vs $843.3 \pm 159 \mathrm{ml} / \mathrm{min} ; \mathrm{p}=0.5)$ and similar central hemodynamics $(\mathrm{p}>0.05)$. At contrary, leg blood flow $(435.5 \pm 27.6 \mathrm{ml} / \mathrm{min}$ vs $590 \pm 54.5 \mathrm{ml} / \mathrm{min} ; \mathrm{p}<0.01)$ and stroke volume $(94.5 \pm 0.5 \mathrm{ml}$ vs $88.31 \pm 0.55 \mathrm{ml} ; \mathrm{p}<0.01)$, were significantly higher for $\mathrm{VC}$ during the 5 th minute of exercise.

Conclusion: These results suggest the negligible role of central command on peripheral circulation during the onset of exercise, while at contrary its role in sustaining peripheral and central hemodynamics during the steady-state exercise appears clear. The mechanism associated with this difference is likely attributable a similar nitric oxide release at the exercise onset for both $\mathrm{ES}$ and $\mathrm{VC}$, while the greater activation of peripheral vasoactive factors (temperature and $\mathrm{pH}$ ) via $\mathrm{VC}$ are likely responsible for the increase peripheral circulation during SteadyState exercise.

\section{EP2 O}

Muscle architecture adaptations to 12-week static stretching training in the lower limbs

\section{S. Longo, E. Cè, A.V. Bisconti, E. Limonta, S. Rampichini, S. Shokohyar, G. Coratella, A. Montaruli, F. Esposito}

Department of Biomedical Sciences for Health, Università degli studi di Milano, Milano, Italy

Purpose: Muscle architecture, one of the main determinant of muscle function and performance, can adapt as a consequence of adequate mechanical stimuli (e.g., strength training). However, whether longterm stretching could lead to architectural changes is still controversial [1]. Therefore, the aim of the present study was to assess possible changes in muscle architecture after 12 weeks of static stretching (SS) in young healthy participants. 
Methods: Twenty-eight participants were randomly divided into 2 groups: stretching (STR, $\mathrm{n}=16,7 \mathrm{~F} / 9 \mathrm{M}$; mean $\pm \mathrm{SD}$ : age $=23.5 \pm$ 1.0 years; stature $=1.75 \pm 0.08 \mathrm{~m}$; body mass $=69.8 \pm 8.9 \mathrm{~kg}$ ) and control $(\mathrm{CTR}, \mathrm{n}=12,6 \mathrm{~F} / 6 \mathrm{M})$; mean $\pm \mathrm{SD}$ : age $=24.2 \pm 2.4$ years; stature $=1.70 \pm 0.06 \mathrm{~m}$; body mass $=65.5 \pm 7.2 \mathrm{~kg}$ ). STR underwent 12 weeks of SS for both pantarflexors (PF) and knee extensors (KE) muscles. CTR did not undergo any training intervention. SS was performed five times/week. One session consisted in 5 sets of 45-s of SS each (15-s of rest in-between). Four exercises were executed: two for PF and two for KE of the right lower limb. Ultrasound images of the medial head of the right gastrocnemius $(\mathrm{GM})$ and vastus lateralis (VL) muscles were obtained at $50 \%$ muscle length before, at six weeks, and after 12 weeks in both groups. Fascicle length (Lf), pennation angle (PA) and muscle thickness (MT) were measured offline by an open source software. A $3 \times 2$ mixedmodel ANOVA was used to assess possible differences over time and between groups in all architectural parameters for each muscle group. Significance was set at $\alpha=0.05$.

Results: Lf, PA and MT did not change significantly in STR over time ( $\mathrm{p}>0.05$ for all parameters) in both PF and KE. No significant differences were found between STR and CTR ( $p>0.05$ for all parameters at any time point) in both $\mathrm{PF}$ and KE. No time $\mathrm{x}$ group interactions were found ( $p>0.05$ for all parameters) in both PF and KE.

Conclusion: 12 weeks of SS did not induce any muscle architectural changes in the mid portion of the GM and VL muscles. These results suggest that common SS exercises for the PF and KE did not provide sufficient mechanical stimuli for structural adaptations of GM and VL, respectively, despite the protocol duration (12 weeks). However, regional adaptations cannot be excluded.

Reference

Simpson CL, Kim BDH, Bourcet MR, Jones GR, Jakobi JM (2017) Stretch training induces unequal adaptation in muscle fascicles and thickness in medial and lateral gastrocnemii. Scand J Med Sci Sports 27(12):1597-1604
TRAINING, PERFORMANCE AND EVALUATION METHODS 3

\section{TP3 K \\ Technical and time-motion indicators, neuromuscular performance, and heart rate impact in elite Italian rugby training}

\section{Lupo $^{1}$, A. N. Ungureanu ${ }^{1}$, P. R. Brustio ${ }^{1}$,} A. Licciardi $^{2}$, G. Boccia ${ }^{1}$, A. Rainoldi ${ }^{1}$

${ }^{1}$ NeuroMuscular Function Research Group, School of Exercise and Sport Sciences (SUISM), Department of Medical Sciences, University of Turin, Italy;

${ }^{2}$ Torino Football Club, Turin, Italy

Purpose: The aim of this study was to monitor elite male Italian rugby training (20 Serie A players, age: $22 \pm 3$ years) with an integrated analysis.

Methods: During three in-season training sessions (S1, S2, S3; $113 \pm 13 \mathrm{~min}$ ), heart rate (HR), internal training load (ITL; Edwards 1993), time-motion (GPS: total distance, duration, mean speed, \% high acceleration and deceleration over corresponding total time, and change of directions $>$ maximal aerobic speed, CoD $>$ MAS, and $>30^{\circ}$ each $\min , \mathrm{CoD}>30^{\circ}$; notational analysis: work-rest ratio) and technical (notational analysis: type and duration of workouts, occurrence of tackles and other minor upper limb contacts) parameters were monitored. Neuromuscular responses were also analyzed (countermovement jump test, CMJ; grip strength, GS) before (PRE) and after (POST) sessions. A $2 \times 3$ (PRE-POST, sessions) ANOVA with repeated measures were applied to evaluate effects for ITLs and GPS (only 9 players), CMJ and GS.

Results: Training sessions consisted of warm-ups without (range 8$13 \mathrm{~min}$ ) and with (0-8 min) ball, $>2$ small groups (38-40 $\mathrm{min})$ and 2 large groups (0-23 $\mathrm{min})$ technical workouts, rugby sevens (0-23 $\mathrm{min})$. No tackles and only minor upper-limb contacts $(25 \pm 7,8-33)$ were counted. No difference between sessions was reported for Edwards' ITL $(488 \pm 24)$. Conversely, differences emerged for total distance $(\mathrm{p}=0.003 ; \mathrm{S} 1,6397 \pm 806 \mathrm{~m}, \mathrm{~S} 2,5044 \pm 735 \mathrm{~m}, \mathrm{p}=0.015 ; \mathrm{S} 1, \mathrm{~S} 3$, $4796 \pm 726 \mathrm{~m}, \quad \mathrm{p}=0.007 ; \mathrm{S} 2, \quad \mathrm{~S} 3, \quad \mathrm{p}=0.004)$, mean speed $(\mathrm{p}=0.017 ; \mathrm{S} 1,61 \pm 8 \mathrm{~m} / \mathrm{min}, \mathrm{S} 3,72 \pm 4 \mathrm{~m} / \mathrm{min}, \mathrm{p}=0.03 ; \mathrm{S} 2$, $66 \pm 4 \mathrm{~m} / \mathrm{min}, \mathrm{S} 3, \mathrm{p}<0.0001)$, high accelerations ( $<<0.0001 ; \mathrm{S} 1$, $5.0 \pm 1.3, \mathrm{~S} 2,7.1 \pm 1.0, \mathrm{p}=0.001 ; \mathrm{S} 1, \mathrm{~S} 3,7.5 \pm 0.9, \mathrm{p}<0.0001)$ and decelerations $(\mathrm{p}<0.0001 ; \mathrm{S} 1, \quad 6.0 \pm 1.7, \quad \mathrm{~S} 2, \quad 8.7 \pm 1.2$, $\mathrm{p}=0.003 ; \mathrm{S} 1, \mathrm{~S} 3,9.4 \pm 1.1, \mathrm{p}=0.001)$, and change of directions (CoD > MAS, $\mathrm{p}<0.0001 ; \mathrm{S} 1,0.9 \pm 0.3, \mathrm{~S} 2,1.4 \pm 0.3, \mathrm{p}=0.001$; $\mathrm{S} 1, \mathrm{~S} 3,1.5 \pm 0.4, \mathrm{p}=0.006 ; \mathrm{COD}>30^{\circ}, \mathrm{p}<0.0001 ; \mathrm{S} 1,17 \pm 3$, $\mathrm{S} 2,21 \pm 1, \mathrm{p}=0.003 ; \mathrm{S} 1, \mathrm{~S} 3,20 \pm 2, \mathrm{p}=0.001)$. Effects emerged also in terms of work-rest ratio $(\mathrm{p}<0.0001 ; \mathrm{S} 1,0.41 \pm 0.01, \mathrm{~S} 2$, $0.83 \pm 0.08, \mathrm{p}<0.0001 ; \mathrm{S} 1, \mathrm{~S} 3,1.42 \pm 0.08, \mathrm{p}<0.0001 ; \mathrm{S} 2, \mathrm{~S} 3$, $\mathrm{p}<0.0001)$. CMJ performances showed differences between session $(\mathrm{p}<0.0001 ; \mathrm{S} 1,35 \pm 2 \mathrm{~cm}, \mathrm{~S} 2,36 \pm 2 \mathrm{~cm}, \mathrm{p}=0.045 ; \mathrm{S} 1, \mathrm{~S} 3$, $37 \pm 2 \mathrm{~cm}, \mathrm{p}=0.006 ; \mathrm{S} 2, \mathrm{~S} 3, \mathrm{p}=0.03)$, whereas GS reported PRE $(579 \pm 20 \mathrm{~N})$-POST $(549 \pm 20 \mathrm{~N})$ effects $(\mathrm{p}=0.002)$.

Conclusions: Despite no effect emerged for ITL, rugby training showed time-motion and neuromuscular effects, highlighting the integrated approach validity. However, further research is strongly recommended.

References

Edwards (1993) Fleet Feet Press, Sacramento

Foster et al (1995) Eur J Appl Physiol Occup Physiol 


\section{TP3 O}

\section{Analysis of the tactical performance in rowing}

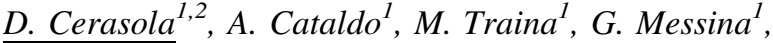 \\ A. Bianco $^{1}$, L. Capranica ${ }^{2}$, A. Palma ${ }^{1}$, D. Zangla ${ }^{1}$ \\ ${ }^{I}$ Department of Movement, Human and Health Sciences, University \\ of Rome "Foro Italico", Italy; \\ ${ }^{2}$ Sport and Exercise Sciences "DISMOT" Research Unit, University \\ of Palermo, Italy
}

Purpose: The aim of this study was to determine the race strategies by elite rowers during world and olympic championships in relation to the final result. In particular, if the elite rowers practice more an offensive or defense strategy. A fast start is a tactically and psychologically advantageous in rowing competition: it allows to monitor and react to any sudden advance from to the other competitors.

Methods: We analyzed the split boats position at every $500 \mathrm{~m}$, according to the competition outcomes during the final race. In particular, we considered the boat position at first $500 \mathrm{~m}(\mathrm{t} 1: 0-500 \mathrm{~m})$, at second $500 \mathrm{~m}(\mathrm{~T} 2: 500-1000 \mathrm{~m})$, at the third (T3: $1000-1500 \mathrm{~m})$ and at last $500 \mathrm{~m}$ (T4: 1500-2000 m) during the last five world rowing championships and the last two Olympic games in heavyweight, lightweight, man and female.

Results: We analyzed 112 race profiles and we noted that $56 \%, 68 \%$ and $78 \%$ of winner boats (gold medal) passed in the first position at T1, T2, T3 respectively. While $87 \%, 96 \%$ and $100 \%$ of medallist boats (first three position) passed in the first three positions at T1, T2, and $\mathrm{T} 3$ respectively.

Conclusions: The results suggest that offensive strategy is the best practice in rowing competition, in particular the boats that obtain the first three positions at T1, T2, T3 have a good chance of winning a medal.

References

Garland SW (2005) An analysis of the pacing strategy adopted by elite competitors in $2000 \mathrm{~m}$ rowing Br J Sports Med 39:39-42

Cerasola D, Cataldo A, Bellafiore M, Traina M, Palma A, Bianco A, Capranica L (2017) Race profile of rowers during the 2014 youth Olympic Games. J Strength Cond Res (Epub ahead of Print)

\section{TP3 O}

\section{Are thresholds obtained from field tests reliable as a race pace index?}

\author{
A. Di Giacomo ${ }^{l}$, F. Tocco ${ }^{l}$ \\ ${ }^{1}$ Sports Physiology Laboratory, University of Cagliari, Italy
}

Purpose: Always more frequently coaches recommend their athletes to perform specific tests to evaluate ventilatory and lactate thresholds. They often complain that thresholds obtained from laboratory tests are not appropriate to plan trainings and do not match with the race pace. We tested the athletes in a more real context i.e. during a training session on field track comparing the values we found with race results. Are thresholds obtained from field tests reliable?

Methods: 32 long distance runners were recruited. They performed 3 tests spaced one week apart. The first test (A) consisted in 6 repeats on $1200 \mathrm{~m}$ with a pace increasing of $15 \mathrm{~s}$ per $\mathrm{km}$ and $50 \mathrm{~s}$ of recovery wearing a portable gas analyzer. The second test (B) consisted of the same trial dosing venous capillary blood lactate with a portable lactate analyzer. The last one (C) consisted in an incremental test with a pace increasing of $10 \mathrm{~s}$ per $\mathrm{km}$ till exhaustion wearing the portable gas analyzer. Athletes wore a GPS and a heart rate monitor. The ventilatory equivalents method was used to identify the first and second ventilatory thresholds (VT1 and VT2), while the individual anaerobic threshold method (IAT) was used to define the lactate thresholds (LT1 and LT2).

Results: All 32 athletes completed the tests. We identified both thresholds in the $72 \%$ of test A, $62.5 \%$ of test B and $78 \%$ of test C; in the other cases the second threshold only was found. VT2 detected with the different methods did not always match.18 athletes took part in 10 and $21 \mathrm{~km}$ races. Athletes' race pace was compared with the estimated values: it matched with VT2 of test A in the $50 \%$ of cases; it corresponded with LT2 found with test B in the $61 \%$ of cases; finally the race pace matched with VT2 of test C in the $44 \%$ of cases. Conclusions: Incremental tests increase the chances to find both thresholds but the VT2 found by this protocol was poorly congruent with the race pace. Technicians should be careful in recommending incremental tests to furnish threshold values for long distance runners. Threshold values estimated with steady state tests seem to be more appropriate.

\section{TP3 O Shoulder mobility in swimmers}

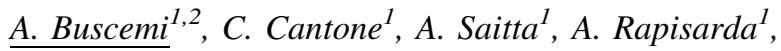

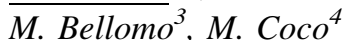

${ }^{1}$ Department of Research, Center Studies of Osteopathy Italian, Catania 95129, Italy;

${ }^{2}$ Horus Cooperative Social, Ragusa 97100, Italy;

${ }^{3}$ Kore University, Enna, Italy;

${ }^{4}$ Department of Biomedical a Biotechnological Sciences, University of Catania, Catania, Italy

Purpose: The shoulder is one of the "engines" of swimmers; its range of mobility (ROM) is essential for a functional and effective stroke. Myofascial force transmission directly connects shoulder with the whole body, through the muscles and their fibrous connective tissue to the skull, the cervical vertebrae, the thorax, the pelvis and the lower limb. The primary objectives of this study were: (1) to evaluate whether the osteopathic manipulative treatment (OMT) could improve shoulder range of motion (ROM); (2) to correlate the ROM with the swimmer's sports performance; (3) to assess, in a group of swimmers, whether an improvement in myofascial force transmission could lead to a reduction in shoulder injuries.

Methods: The sample consisted of 26 competitive swimmers randomized in 2 groups made of 13 subjects: one group was treated with osteopathic techniques, while the control group did not receive any treatment.

The ROM was measured using three dry-land tests (i.e. Circling Test, Behind Elevation Arms test and Up Arms test); the number of strokes was measured on 200-m crawl (without diving; three underwater dolphin kicks, both at start and turns; competition speed performance).

Measurement tests, followed by four OMTs were administered to the athletes on a weekly basis. 15 days after the last OMT, all tests were repeated to determine whether the treatment had maintained its effectiveness over time. Treatments, as well as measurements, were blindly applied by an osteopath and another operator, respectively.

Results: The most relevant data is that obtained in dry-land tests in which athletes significantly improved the ROM of the scapulohumeral joint $(\mathrm{P}<0.0001)$, thus demonstrating that the cervicothoracic junction is closely connected with the pectoralis-clavicle-axillary one and that the OMT can improve fascial plans sliding in this area. Nonstatistically significant results were obtained from in-water tests. 
Discussion: The study demonstrated the efficacy of the OMT on joint mobility. So, we hypothesize that osteopathy could be seen as an useful integrative treatment to athletic training. This would help athletes, in our case swimmers, to increase their performance and reduce the probability of injuries.

In our opinion, several variables conditioned the stroke count: athletes' psycho-physical condition; ongoing agonistic activity; stress predisposition during testing; etc.

Furthermore, stroke count does not represent a true measurement unit.

Keywords: Range of mobility, Muscles, Swimmer

References

Struyf F, Tate A, Kuppens K, Feijen S, Michener LA (2017) Musculoskeletal dysfunctions associated with swimmers' shoulder. Review. Br J Sports Med 51(10):775-780

Parravicini G, Bergna A (2017) Biological effects of direct and indirect manipulation of the fascial system. Narrat Rev. J Bodyw Mov Ther 21(2):435-445

\section{PHYSICAL EXERCISE AS PREVENTION AND THERAPY 2}

\section{EH2 K \\ Diffusion tensor imaging reveals training-induced changes in calf muscles}

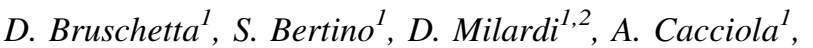 \\ G. Cutroneo ${ }^{1}$, G. Rizzol, G. Basile ${ }^{1}$, A. Duca ${ }^{2}$, D. Buda ${ }^{1}$,

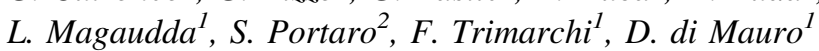 \\ ${ }^{1}$ Department of Biomedical, Dental Sciences and Morphological \\ and Functional Images, University of Messina, 98125, Messina, Italy; \\ ${ }^{2}$ IRCCS Centro Neurolesi “Bonino Pulejo", S.S. 113, Via Palermo, \\ C.da Casazza, 98124, Messina, Italy
}

Purpose: Diffusion tensor imaging is (DTI) is a sensitive magnetic resonance technique to investigate changes in tissue microstructure and a useful tool for macroscopic muscle architecture visualization. The purpose of this study is to investigate how diffusion parameters changes in the human calf muscles after a 3-months strength training protocol.

Methods: Ten young men were enrolled for a strength training protocol aimed at increase size and strength of the medial of the medial (GCM), lateral gastrocnemius (GCL) and soleus (SL). The subjects underwent exercise three times a week, with at least $24 \mathrm{~h}$ between training sessions, for a period of three months. Diffusion weighted magnetic resonance images were acquired at the beginning of the training period $\left(\mathrm{T}_{\mathrm{PRE}}\right)$ and at three months $\left(\mathrm{T}_{\mathrm{POST}}\right)$ using a 3-T scanner. The mean fractional anisotropy (FA), mean diffusivity (MD), $\lambda_{1}, \lambda_{2}, \lambda_{3}$ of all included voxels were assessed, then, tractography was employed to visualize muscle fibres.

Results: We found significant differences in $\lambda_{1}, \lambda_{2}, \lambda_{3}$, MD values and volumes between $\mathrm{T}_{\mathrm{PRE}}$ and $\mathrm{T}_{\mathrm{POST}}$ in all the examined muscles both for the left and right side. No significant differences were highlighted for FA. Muscle volume increase was significant in GCM and GCL. $\lambda_{1}$ increased significantly between $T_{P R E}$ and $T_{P O S T}$ in GCM, GCL but not in SL; $\lambda_{2}$ and $\lambda_{3}$ reached significant increase in all the three muscles. MD increased significantly in GCM. GCL and SL. No significant differences were highlighted between right and left sides neither at $\mathrm{T}_{\mathrm{PRE}}$ nor at $\mathrm{T}_{\mathrm{POST}}$.
Conclusion: Muscle volumes, MD, and eigenvalues variations suggest a training-induced increase of intracellular and extracellular volume. Thus, DTI allows for an accurate assessment of microstructural changes of the muscles in response to training being a promising tool in sport medicine.

\section{References}

Froeling M, Oudeman J, Strijkers GJ, Maas M, Drost MR, Nicolay K, Nederveen AJ (2015) Muscle changes detected with diffusion-tensor imaging after long-distance running. Radiology 2:548-562

Okamoto Y, Kunimatsu A, Miki S, Shindo M, Niitsu M, Minami M (2008) Fractional anisotropy values of calf muscles in normative state after exercise: preliminary results. Magn Reson Med Sci 7:157-162

\section{$40 \mathrm{EH} 2 \mathrm{O}$ \\ Barriers of being active: differences between two generations}

\section{B. Tosi, M. Rosselli, G. Mascherini, C. Petri, G. Galanti, P.A. Modesti}

Unità di Medicina dello Sport e dell'Esercizio, Dipartimento di Medicina Sperimentale e Clinica, Università degli Studi di Firenze

Purpose: It is well known that regular exercise reduces the risk of fatal and non-fatal disease. Physical activity is also one of the seven metrics included in the definition of Ideal Cardiovascular Health. Physical inactivity and sedentary behaviors are the other side of the same coin and are nowadays included among principal risk factors for coronary artery disease, cardiovascular events and mortality. In order to promoting physical activity, it is important to have a thorough understanding of physical activity barriers and facilitators. The study aimed to investigate the principal perceived barriers to exercise in young and adult populations.

Methods: This is a cross-sectional study conducted during 2017-2018 in two settings: a high school (70 students, $19.1 \pm 0.4$ years old) and the Rectorate of University of Florence (106 adults, $48.4 \pm 12.2$ years old). The participants underwent a questionnaire that consisted of three parts: demographic data and physiological anamnesis; Barriers to Being Active Quiz (BBAQ) and Mediterranean Diet Score assessment. Weight, waist and hip circumferences and blood pressure values were measured according to standardize protocols.

Results: Prevalence of overweight (BMI $>25 \mathrm{~kg} / \mathrm{m}^{2}$ ) was $17 \%$ and $42 \%$ among woman of men working in University, being $4 \%$ among both female and male students. Mean adherence score of Mediterranean diet was $34 \pm 3$ among adults and $32 \pm 5$ among students without sex differences in both groups. Sedentary habits were present in the $35 \%$ of adults and $24 \%$ of all students enrolled. Lack of time for exercise was the most frequently reported barrier for adults followed by lack of energy and laziness. These barriers were well represented in young population too, especially in girls with a barrier score $\geq 5$ higher compared to boys ( $\mathrm{p}<0.0019$ ). Surprisingly, fear for injuries was represented only in adult and young male.

Conclusions: Exercise professionals should be aware of the barriers that patients can face during exercise prescription and be able to contrast them with useful individual strategies. Furthermore, analysis of young people life-style habits can help finding social strategies to promote healthy behaviors. Prevention polices to promote physical activity should focus on young girls as a specific target for prevention. References

Mailey EL, Huberty J, Dinkel D, McAuley E (2014) Physical activity barriers and facilitators among working mothers and fathers. BMC Public Health 14. 
Petter M, Blanchard C, Kemp KAR, Mazoff AS, Ferrier SN (2009) Correlates of exercise among coronary heart disease patients: review, implications and future directions. Eur J Cardiovasc Prev Rehabil 16(5):515-526. https://doi.org/10.1097/hjr.0b013e3283299585

\section{$41 \mathrm{EH} 2 \mathrm{O}$}

\section{A.C.T.I.V.E. AGEING (Adapted Controlled Training} Individualised through Verification in the Elderly): an innovative comprehensive multifactorial evaluation for the planning and monitoring of Adapted Physical Activity in the elderly

\section{D'Amico ${ }^{1,2}$, D. Bondi ${ }^{1}$, E. Kinel ${ }^{2,3}$, P. Roncoletta ${ }^{2}$, V. Verratti ${ }^{1}$, C. Doria ${ }^{4}$, T. Pietrangelo}

${ }^{1}$ Functional Evaluation Laboratory, Department of Neuroscience, Imaging and Clinical Science, University "G. D'Annunzio" ChietiPescara, Italy;

${ }^{2}$ SMART Lab (Skeleton Movement Analysis and Advanced Rehabilitation Technologies) Bioengineering \& Biomedicine Company Srl, Pescara, Italy;

${ }^{3}$ Department of Rheumatology and Rehabilitation, Clinic of Rehabilitation, University of Medical Sciences, Poznan, Poland; ${ }^{4}$ Department of Biomedical Sciences for Health, University of Milan, Italy

Purpose: The project A.C.T.I.V.E. AGEING herein presented is part of an extended ambitious interdisciplinary, multifactorial project started by our group. The project aims to bring back to more active and physically efficient life, elderly people. The general framework is to build individualised adapted physical activities (APA) to contrast sedentary lifestyle, sarcopenia and low-grade chronic inflammatory status (Inflammaging) to prevent immobility syndrome in the elderly. Individualised APA for the elderly is necessary to avoid the overwhelming physical-psychological stress that could lead to failure with reduced functional efficiency, increased pain, subsequent lack of motivation and abandonment of training programmes.

Methods: To achieve the "proper individualised APA" and to monitor its effect on the elderly a number of quantitative measurements are planned:

1. Anthropometry including plicometry and measurement of circumferences.

2. Battery of Validated Functional Tests:

a. Five Times Sit-to-Stand Test (FTSST) and Timed Up-andGo test (TUG).

b. Maximal Voluntary Contraction (MVC) of quadriceps.

3. Comprehensive Multifactorial Biomechanical Analysis (CMBA) of full 3D skeleton Posture and Movement using new technological Opto-Electronic Stereophotogrammetric devices in conjunction with Baropodometric and Surface Electro-Myographic devices [1, 2]

4. Bio-histo-chemical analysis through muscle biopsy to measure exercise-associated muscle structure changes.

Twenty participants (age $71.77 \pm 4.06$ years) were subdivided randomly into 2 groups: controls (n.10 subjects no APA); resistance training APA supplemented with neuro-muscular electrical stimulation (10 subjects). Two-way ANOVA for repeated measures was used to analyse differences between groups and over pre (T0)-and post (T1) after 12 weeks APAs.

Results: The groups presented no differences at T0, while as expected the APA group showed improvements at T1 in: FTSST, TUG, MVC,
CMBA and in muscle structure. Conversely, 12 weeks period of APA revealed to be insufficient to find significant changes in anthropometry. All participants reported an optimal compliance with planned level of APA.

Conclusions: The described general framework proved to be effective to plan and monitor "proper individualised APA". It demonstrates the importance to collect multifactorial data and integrate them into a unique framework, providing a real added value in the detailed comprehension of functional and biomechanical phenomena.

\section{References}

D'Amico M, Kinel E, Roncoletta P (2017) Normative 3D optoelectronic stereo-photogrammetric posture and spine morphology data in young healthy adult population. PLoS One 12: e0179619. https://doi.org/10.1371/journal.pone.0179619

D'Amico M et al (2018) An innovative comprehensive multifactorial evaluation of 3D full skeleton posture and spine functional mobility for the quantitative morpho-functional characterization for APA in the elderly. Present X Congresso Nazionale SISMES, 5-7 October 2018, Messina

\section{EH2 O \\ Gender differences in physical fitness and its relationship to sexual health in older adults}

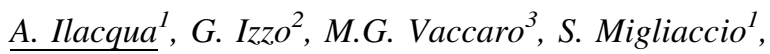 \\ C. Baldari $^{1}$, L. Guidetti ${ }^{1}$, A. Aversa ${ }^{2}$, G.P. Emerenziani ${ }^{2}$
}

${ }^{1}$ Dipartimento di Scienze Motorie, Umane e della Salute, Università degli Studi di Roma "Foro Italico", Roma, Italia;

${ }^{2}$ Dipartimento di Medicina Sperimentale e Clinica, Università degli Studi di Catanzaro "Magna Graecia", Catanzaro, Italia;

${ }^{3}$ Dipartimento di Scienze Mediche, Università degli Studi "Magna Graecia" di Catanzaro, Catanzaro, Italia

Purpose: Human sexuality has been explained in different domains. In the elderly, there is a growing literature on the correlation between sexuality and cognitive functions, but there is no data on the relationship between sexuality and physical performance.

The aim of the study was to analyze the correlation between sexual well-being and physical performance in elderly subjects.

Methods: 64 subjects were recruited (age $=71.5 \pm 5.0$ years, 31 males, 33 females). Sexuality was assessed by administering two questionnaires: Sexual Functioning Questionnaire (CSFQ-M/F) and Sexual Attitude Scale (SAS). Physical performance was assessed by handgrip test (HG), short physical performance battery (SPPB), $30 \mathrm{~s}$ chair stand test, (CST30 s), time up and go test (TUG) and 2 min step test (ST). The correlation analysis between sexual well-being and physical efficiency tests were performed for both genders independently. The significance level was established equal to $\mathrm{p} \leq 0.05$.

Results: In females, CSFQ-14 was negatively correlated to TUG ( $\mathrm{p}<0.01 ; \mathrm{r}=-0.522)$ but positively to CST $(\mathrm{p}<0.01, \mathrm{r}=0.478)$, to $4 \mathrm{~m}$ WS $(\mathrm{p}<0.05 ; \mathrm{r}=0.443)$ and to ST $(\mathrm{p}<0.05 ; \mathrm{r}=0.456)$. SAS was positively to CST30 $\mathrm{s}(\mathrm{p}<0.01, \mathrm{r}=0.392)$.

In males, CSFQ-14 was negatively correlated to CST30 s ( $p<0.05$; $\mathrm{r}=-0.450)$ but positively to TUG $(\mathrm{p}<0.05 ; \mathrm{r}=0.548)$ and to $4 \mathrm{~m}$ WS ( $<<0.05 ; r=0.383$ ). No correlation were found between SAS and all the others variables.

Conclusions: Our preliminary study suggests that resistance of the lower limbs, speed of walking and cardiovascular efficiency may positively influence sexual functions in the elderly, especially in older women. Coordination and power do not seem to affect sexual function. Assessment of sexual hormones is recommended for promoting motor performance. 


\section{Reference}

Boss L, Kang DH, Bergstrom N, Leasure JL (2015) Endogenous sex hormones and cognitive function in the elderly. Aging Clin Exp Res 27:515-521

\section{EH2 O \\ Social dance improves mood and fitness performance in older adults}

\author{
M.G. Vaccaro $^{1}$, G. Izzo ${ }^{2}$, C. Baldari ${ }^{3}$, L. Guidetti $^{3}$,

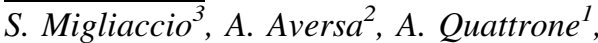 \\ G.P. Emerenziani ${ }^{2}$
}

${ }^{I}$ Dipartimento di Scienze Mediche, Università degli Studi "Magna Graecia" di Catanzaro, Catanzaro, Italia;

${ }^{2}$ Dipartimento di Medicina Sperimentale e Clinica, Università degli Studi "Magna Graecia" di Catanzaro, Catanzaro, Italia;

${ }^{3}$ Dipartimento di Scienze Motorie, Umane e della Salute, Università degli Studi di Roma "Foro Italico", Roma, Italia.

Purpose: Correlation between aging processes, neuropsychological and physical performances in older adults has been well established. However, the impact of regular social dance practice on cognitive functions and sexual health in older age is still poorly studied. Thus, this study aims to investigate whether a 6 months of social dance practice might influence specific cognitive functions and/or mood characteristics, fitness performance and sexual health in older adults. Methods: In this study, 25 participants (age $=71.0 \pm 5.6 ; 8$ males, 17 females) were enrolled from the dance school "NonSoloLiscio" of Catanzaro. Subjects were evaluated at the beginning (T0) and after 6 months of social dance practice (T6). In both session tests, after anthropometric examination, subjects performed fitness tests: hand grip (HG), $30 \mathrm{~s}$ CST (CST30 s), balance (Bal), chair stand test (CST), $4 \mathrm{~m}$ walking speed (4mWS), 2 min step test (ST), time up and go (TUG,); psychological tests: mini mental state examination (MMSE), prospective (PM) and retrospective memory (RM) questionnaire (PMRQ), state-trait anxiety inventory form Y (STAI Y-1; STAIY-2), geriatric depression scale (GDS); and sexuality questionnaires: sexual attitude scale (SAS) and changes in sexual functioning questionnaire (CSFQ14).

Results: After 6 months of dance practice, percent of fat mass (pFM) significantly decreased $(\mathrm{p}<0.01)$ while fat free mass $(\mathrm{FFM})$ $(p<0.01)$ significantly increased in both genders. Moreover, significant main effect of time on CST $(p<0.01)$, GS $(p<0.05)$ and TUG $(\mathrm{p}<0.05)$ were found.

In addition, both female and male participants showed a significant improvement in PRMQr $(\mathrm{p}<0.05)$ and STAI Y-2 $(\mathrm{p}<0.05)$ score compared to T0. No differences were found between pre and post intervention in sexual health variables.

Conclusions: Our preliminary results suggest that the practice of social dance in older adults might positively influence body composition in both genders and might also increase fitness performance. Moreover, data showed that social dance might positively influence both retrospective memory and anxiety state. Instead, no effects on sexual health were found after 6 months of social dance practice.

Acknowledgments: We thanks the subjects of "NonSoloLiscio" dance association to have participated to this study.

References

Marquez DX, Wilson R, Aguiñaga S et al (2014) Regular Latin dancing and health education may improve cognition of late middleaged and older latinos. J Aging Phys Act 25:482-489
Zhang X, Ni X, Chen P (2014) Study about the effects of different fitness sports on cognitive function and emotion of the aged. Cell Biochem Biophys 70:1591-1596

\section{PHYSICAL EDUCATION AND SPORT PEDAGOGY 2}

\section{PE2 K \\ The development of motor skills by a physical education programme in preschool children: a preschool-based controlled trial}

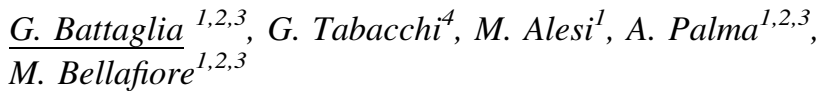

${ }^{1}$ Department of Psychological, Pedagogical and Educational

Sciences, University of Palermo, Sicily, Italy;

${ }^{2}$ Sport and Exercise Sciences Research Unit, University of Palermo, Sicily, Italy;

${ }^{3}$ Regional Sports School of CONI Sicilia, Sicily, Italy;

${ }^{4}$ Department of Sciences for Health Promotion and Mother Child Care "G. D'Alessandro"

Purpose: Unlike other European countries, Italian kindergartens do not include the physical education teacher in the school organic personnel. This is frequently associated with the lack of opportunities to perform physical education by preschool children. The aim of this study was to analyse the effects of a specific physical education program (PEP) on the quotient of gross motor development (QGMD) in preschool children.

Methods: We conducted a school-based non-randomised controlled trial. It involved 119 children, clustered in a control group (CG, $\mathrm{n}=29$, age $52.1 \pm 8.65$ months; height $1.1 \pm 0.07 \mathrm{~m}$, body weight $19.2 \pm 5.55 \mathrm{~kg}$, body mass index (BMI) $16.9 \pm 3.16$ ) and an intervention group (IG, $\mathrm{n}=90$, age $57.4 \pm 9.42$ months; height $1.1 \pm 0.06 \mathrm{~m}$, body weight $19.3 \pm 3.65 \mathrm{~kg}$, BMI $16 \pm 1.75)$. Participants were assessed for locomotor ability and object control skills using the Italian version of GMD test, before and after the experimental period.

Results: IG increased the locomotor object-control skills and QGMD by $24.4 \%, 9.7 \%$ and $10.4 \%$, in response to PEP. The mean difference of the QGMD between CG and IG, in the pre and post period, was significantly higher for the IG $(11.3$ vs $3.2, p=0.0082)$. Specific items did not increase in the CG, while a highly significant improvement was observed in all the items in the IG, in response to PEP.

Conclusions: The specific PEP improved fundamental motor skills in preschool children. Consistently with the growing research (Zeng et al. 2017), interventions based on scheduled physical exercises ensure health benefits for preschool children.

\section{Reference}

Zeng N, Ayyub M, Sun H, Wen X, Xiang P, Gao Z (2017) Effects of physical activity on motor skills and cognitive development in early childhood: a systematic review. Biomed Res Int 2017:2760716 


\section{PE2 O}

\section{Geographical differences in physical activity, motor performance, and overweight and obesity status in Italian children: cities comparison}

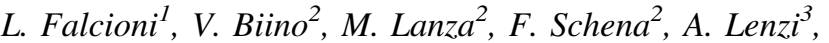 \\ S. Migliaccio ${ }^{1}$, C. Baldari ${ }^{1,4}$, L. Guidetti ${ }^{1}$ \\ ${ }^{1}$ Department of Movement, Human and Health Sciences, University \\ of Rome "Foro Italico", Italy; \\ ${ }^{2}$ Department of Neurosciences, Biomedicine and Movement Sciences, \\ University of Verona, Italy; \\ ${ }^{3}$ Department of Experimental Medicine, University of Rome \\ "Sapienza", Italy; \\ ${ }^{4}$ eCampus University, Novedrate (CO), Italy
}

Purpose: Childhood overweight and obesity, within 7 and 10 years of age, is dramatically increased in Italy and is around $36 \%$. Thus, aim of the study was to examine whether there are differences in overweight and obesity prevalence, physical activity levels, and motor coordination between school age children from Northern (Mantova and Verona) and Central (Roma) Italy. Also, the walkability of the different areas was investigated.

Methods: 391 children (198 from the Center and 193 from the North) aged 8-11 years, participated in the study. Anthropometric assessments were performed and physical activity level was measured through PAQ-C questionnaire. The KTK test battery was selected to evaluate children's motor coordination. Finally, walkability measurements were collected using the Walk Score $\mathbb{R}$ (http://www. walkscore.com). Factorial ANOVA and appropriate post-hoc analyses were performed.

Results: The prevalence of overweight and obesity was similar between North (overweight 13.5\%; obesity 11.9\%) and Center (overweight $13.6 \%$; obesity $16.7 \%$ ) of Italy. Overall, children in the North had a marginally lower BMI (North $17.9 \pm 3.5$ vs Center $\left.18.6 \pm 3.6 ; \mathrm{F}_{1,386}=3, \mathrm{p}=0.08\right)$, a higher significant level of physical activity (North $2.6 \pm 0.6$ vs Center $2.3 \pm 0.7 ; \mathrm{F}_{1,380}=19, \mathrm{p}<$ 0.01 ) and motor coordination (North $102.2 \pm 12.5$ vs Center $80.8 \pm$ $\left.10.4 ; \mathrm{F}_{1,387}=378, \mathrm{p}<0.01\right)$. Children attending schools located in an area with a high walking index showed lower BMI (Walkable $18.6 \pm$ 3.4 vs Car dependent $\left.18.8 \pm 4.1 ; \mathrm{F}_{1,189}=4, \mathrm{p}<0.05\right)$ and greater motor coordination (Walkable $81.8 \pm 10.4$ vs Car dependent $78.1 \pm$ $10.1 ; \mathrm{F}_{1.189}=6, \mathrm{p}<0.05$ ). In particular, overweight and obese girls from car dependent areas showed a higher BMI (Walkable $22.1 \pm 1.9$ vs Car dependent $25.3 \pm 4.1 ; \mathrm{p}<0.05)$ and a worse coordination level (Walkable $73.0 \pm 8.2$ vs Car dependent $61.7 \pm 4.4 ; \mathrm{p}<0.01$ ). Conclusions: Children from North of Italy showed a better profile in terms of BMI, physical activity level, and motor coordination. Our results strongly suggest that it might be necessary to design more walkable cities in order to reduce the prevalence of overweight and the lack of coordination in Italian children.

\section{References}

Kiphard EJ, Schilling F (2007) KörperkoordinationsTestfür Kinder. 2. Uberarbeitete und erganzte Auflage. Beltz Test GmbH, Weinheim Kowalski KC, Crocker PRE, Donen RM (2004) The Physical Activity Questionnaire for Older Children (PAQ-C) and Adolescents (PAQ-A) Manual. College of Kinesiology, University of Saskatchewan, pp 1-37

\section{PE2 O \\ Key Inclusive Sport System}

\author{
P. A. Cassalia ${ }^{1}$, A. Arcigli ${ }^{2}$
}

${ }^{1}$ Coordinatore staff tecnico CONI Messina;

${ }^{2}$ Delegato CONI Messina

Purpose: From the results obtained in 30 years of research and action in schools, from intense olympic and paralympic experiences and from a proper and virtuous use of european funds the Key Inclusive Sport System (KISS) for the relaunch of italian sport and the setting of new didactic and pedagogical scenaries for the teaching of motor activities in primary e secondary school is born.

Method: The project makes a proper use of the Cassalia Method-A Study in Movement (SiM), widely tried and documented through various years

Methods: This method, fueled by a social-educational-sportive reason, aims to create an environment focused on the awakening of the individual curiosity, urging the motivation to learn and the ability to cooperate, developing the skills of every student by making school more attractive. SiM, applicable in various fields, by using the strong stimulus of physical and recreational activity, allows the young pupils for a dynamic and motivational learning, intervening in context of social marginality where the traditional teaching meets serious barriers to prove itself useful and where the most culturally challenged students need a proper education more than ever.

Results: This method intends to focus the attention on the educational system, on the social inclusion and on the sport intended as a way to mitigate school drop-out and cyber-bullying.

A brand-new physical education in line with an independent didactic, the official MIUR and CONI guidelines and with the European Community's program needs an elevated amount of professionals who can characterize such plans at the best of their possibilities, being the answer to an increasingly demand of renewal through a complex process of teaching and learning aimed at a more effective education of young students. School needs to be a more welcoming place as to obtain more interest in projects involving an ever growing demand for inclusion and motivation. In this archetype, the figure of a tutor shaped by the KISS program is to be considered one of the most useful.

Conclusions: Good method and lots of training have been the subjects of the research, included by outer experts as a part of innovational methodology in PON projects, senior thesis, seminars and heart of short productions in numerous Italian companies, loved by thousands of students.

\section{Reference}

On YouTube, the documentary film "La banda del paloggio Storia di un metodo" and https://youtu.be/TqbseCBrUXc 


\section{PE2 O}

\section{Potentiated physical education interventions are effective in ameliorating physical fitness of Italian primary schoolchildren}

\author{
R. Codella ${ }^{1}$, C. Galvani ${ }^{2}$, M. Milani ${ }^{2}$, M. Vandoni $^{3}$, \\ L. Correale ${ }^{3}$, MV. Puci ${ }^{3}$, F. Togni ${ }^{4}$, F. Casolo ${ }^{2}$, A. Passi $^{4}$, \\ C. Orizio ${ }^{5}$, G. Alberti ${ }^{1}$, C. Montomoli ${ }^{3}$, A. La Torre ${ }^{1}$ \\ ${ }^{1}$ Università degli Studi di Milano; \\ ${ }^{2}$ Università Cattolica del Sacro Cuore, Milano; \\ ${ }^{3}$ Università di Pavia; \\ ${ }^{4}$ Università degli Studi dell'Insubria; ${ }^{5}$ Università degli studi di \\ Brescia
}

Purpose: School-based PE programs are poor nationwide and they need to be potentiated against a rising prevalence of chronic diseases and physical inactivity. Our aim was to evaluate the effectiveness of 40 versus 20 physical education (PE) classes on physical fitness of a cohort of northwestern Italian primary schoolchildren.

Methods: 5751 schoolchildren aged 6-11 years were studied between November 2017 and May 2018 from 25 primary schools of Lombardy. Pupils underwent either a control group $(\mathrm{CON}, \hat{\jmath}=2553$; $q=2864$ ) or an experimental group (PLUS, $\hat{o}=158$; $q=176)$ in which they attended 20 or 40 PE classes, respectively. Sixty-minute classes were delivered once a week (CON) or biweekly (PLUS) by qualified PE teaching assistants through an approximate school semester. Anthropometrics, cardiorespiratory (six-minute walking test, 6MWT), musculoskeletal (standing broad jump, SBJ) and motor fitness $(4 \times 10 \mathrm{~m}$ shuttle run test, SRT) were evaluated at baseline and upon completion of the study. Wilcoxon matched-pairs signed rank test was performed.

Results: At the end of the study, PLUS group revealed a better distribution of BMI categories (under weight UW $\Delta_{\text {post-pre }}=-5.4 \%$; normal weight NW $\Delta_{\text {post-pre }}=+8.9 \%$; overweight OW $\Delta_{\text {post-pre }}=$ $-3.6 \%$ ) compared to controls (UW $\Delta_{\text {post pre }}=-0.4 \%$; NW $\Delta_{\text {post- }}$ pre $=+1.6 \% ;$ OW $\left.\Delta_{\text {post-pre }}=-1 \%\right)$. PLUS children improved almost all fitness performances by a greater extent (6MWT: pre $606.8 \pm 104 /$ post $\quad 644.5 \pm 93.5 \mathrm{~m}, \quad \mathrm{p}<0.0001 ; \quad$ SBJ: $\quad$ pre $119.1 \pm 25.3 /$ post $124.8 \pm 25.9 \mathrm{~cm}, \quad \mathrm{p}<0.0001 ; \quad \mathrm{SRT}$ : $\quad$ pre $14.9 \pm 2.5 /$ post $13.8 \pm 1.9 \mathrm{~s}, \mathrm{p}<0.0001)$ with respect to $\mathrm{CON}$ children (6MWT: pre $611.4 \pm 102.1 /$ post $623.6 \pm 92.4 \mathrm{~m}$, $\mathrm{p}<0.0001 ;$ SBJ: pre $116.8 \pm 25.3 /$ post $123.4 \pm 24 \mathrm{~cm}, \mathrm{p}<0.02$; SRT: pre $14.8 \pm 2.4 /$ post $14.3 \pm 2 \mathrm{~s}, \mathrm{p}<0.0001)$. Means and SD.

Conclusions: This design confirms the need to implement wellstructured, school-based PE programs. Particularly, higher frequency of PE classes is advocated in order to ameliorate health-related physical fitness competence.

References

ATS (2002) Am J Respir Crit Care Med 166(1):111-117

Artero EG et al (2011) Int J Sports Med 32(3):159-169

\section{EXERCISE PHYSIOLOGY 3}

\author{
48 EP3 K \\ RCP and deoxy-BP change at unison \\ during longitudinal interventions
}

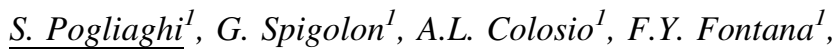 \\ L. Ferrari ${ }^{\text {, }}$ J.M. Kowalchuk ${ }^{2,3,4}$
}

${ }^{I}$ Department of Neuroscience, Biomedicine and Movement Science, University of Verona, Verona, Italy;

${ }^{2}$ Canadian Centre for Activity and Aging;

${ }^{3}$ School of Kinesiology;

${ }^{4}$ Department of Physiology and Pharmacology, The University of Western Ontario, London, Ontario, Canada

Purpose: We showed previously that oxygen uptake $\left(\dot{\mathrm{V}}_{2}\right)$ at the respiratory compensation point (RCP) and at the near-infrared spectroscopy (NIRS)-derived muscle deoxygenation breakpoint (deoxy$\mathrm{BP})$, measured during ramp-incremental cycling, were not different $[1,2]$. Furthermore, we suggested that these indexes represent, respectively, the integrated vs the local physiological expression of the same underlying phenomenon i.e., the heavy-to-severe exercise intensity boundary.

To test the hypothesis that the $\dot{\mathrm{VO}}_{2}$ at $\mathrm{RCP}$ and deoxy-BP change in unison during a longitudinal study, in turn corroborating the idea of a common metabolic stimulus.

Methods: Healthy, adult males (n, 65; age, $43 \pm 21$ years [mean \pm $\mathrm{SD}$; peak VO2, $41 \pm 11 \mathrm{ml} \mathrm{kg}^{-1} \mathrm{~min}^{-1}$ ) performed two incremental cycling tests to exhaustion $\left(20 \mathrm{~W}\right.$ baseline $+15 / 30 \mathrm{~W} \mathrm{~min}^{-1}$ increment) before (PRE) and after (POST) a range of interventions (control, aerobic training, strength training, acute non-metabolic fatigue) that resulted in either no change $(+0.1 \pm 1.5 \%$;, 25$)$, a reduction $(-8.4 \pm 5.8 \% ; \mathrm{n}, 11)$ or an increase $(+11.4 \pm 11.5 \% ; \mathrm{n}$, 29) in peak $\mathrm{VO}_{2}$. Gas exchange variables were determined breath-bybreath and muscle deoxygenation of the right vastus lateralis was monitored non-invasively by pulsed-wave, multiple-distance NIRS. The $\mathrm{VO}_{2}$ corresponding to the RCP and the deoxy-BP (double linear function fitting) were identified and compared by RM ANOVA and Pearson's correlation. The correspondence between the absolute changes of RCP and deoxy-BP was evaluated by paired $t$ test and Pearson's correlation.

Results: Individual values $\mathrm{VO}_{2}$ at $\mathrm{RCP} v s$ deoxy-BP were not different $(p=0.48$ PRE; $p=0.12$ POST) and were highly correlated $(r=0.92, p<0.001$ PRE; $r=0.93, p<0.001$ POST $)$. The individual absolute changes between PRE and POST measures of RCP $\left(-12 \pm 196 \mathrm{~mL} \mathrm{~min}^{-1}\right)$ and deoxy-BP $\left(31 \pm 276 \mathrm{~mL} \mathrm{~min}^{-1}\right)$ were not different $(\mathrm{p}=0.14)$ and were correlated positively $(\mathrm{r}=0.59$, $\mathrm{p}<0.001)$.

Conclusions: RCP and deoxy-BP occur at an identical metabolic intensity $\left(\mathrm{VO}_{2}\right)$ and the "change" in the RCP and deoxy-BP consequent to the experimental intervention were not different. The close relationship between the RCP and deoxy-BP suggests that a mechanistic (metabolic) link exists between the two variables.

\section{References}

Keir DA, Fontana FY, Robertson TC, Murias JM, Paterson DH, Kowalchuk JM, Pogliaghi S (2015) Med Sci Sports Exerc 47(9):1932-1940

Fontana FY, Keir DA, Bellotti C, De Roia GF, Murias JM, Pogliaghi S (2015) J Sci Med Sport 18(5):590-595 


\section{EP3 O}

\section{Arterial blood gas analysis in breath-hold divers at depth}

\author{
G. Bosco ${ }^{1}$, A. Rizzato ${ }^{1}$, L. Martani ${ }^{2}$, S. Schiavo ${ }^{2}$, \\ E. Talamonti ${ }^{2}$, M Paganini ${ }^{1}$, G. Garetto ${ }^{3}$. \\ ${ }^{1}$ Department of Biomedical Sciences, University of Padova, Italy; \\ ${ }^{2}$ Master level II in Diving and Hyperbaric medicine, University \\ of Padova Italy \\ ${ }^{3}$ ATIP Hyperbaric medicine Center, Padova, Italy
}

Purpose: This is the first observational study to verify real changes in arterial blood gases during a breath-hold dive in well trained freedivers at depth.

Methods: Six of eight well trained, healthy breath-hold divers regularly completed this study $(46.66 \pm 9.30$ years, $78.16 \pm 10.66 \mathrm{~kg}$ and $1.76 \pm 0.04 \mathrm{~m}$ ). All the divers performed a sled-assisted breathhold dive up to 40 meters in a swimming pool. Prior to submersion, an arterial cannula in the radial artery of the non-dominant limb was positioned. Withdrawals took place before the dive, at 40 meters depth and after the emersion measuring $\mathrm{PH}, \mathrm{pCO}_{2}(\mathrm{mmHg}) \mathrm{pO}_{2}$ $(\mathrm{mmHg}) \mathrm{HCO}_{3}(\mathrm{mmol} / \mathrm{L})$, so2 $(\%)$, tco2 $(\mathrm{mmol} / \mathrm{L})$ and lactate.

Results: Friedman test used to compare data collected in the different time points (PRE, at depth and POST) and $t$ test (PRE and at depth) showed non-significant differences for the parameters but $\mathrm{pO}_{2}$ increased at the bottom and reduced after the dive in four of six divers. However, a statistically significant difference $(p<0.01)$ was observed in comparing PRE $(6.433 \pm 2.313 \mathrm{mg} / \mathrm{dL})$ vs POST $(19.50 \pm 5.056 \mathrm{mg} / \mathrm{dL})$ lactate values.

Conclusions: Our results are consistent with those predicted in the literature for 4 of 6 divers. The reason of this discrepancy needs to be speculated. The increase in lactate is justified both by anaerobic metabolism and peripheral vasoconstriction due to diving reflex.

References

Lindholm P, Lundgren CE (2009) The physiology and pathophysiology of human breath-hold diving. J Appl Physiol 106(1):284-292 Muth CM, Radermacher P, Pittner A, Steinacker J, Schabana R, Hamich S, Paulat K, Calzia E (2003) Arterial blood gases during diving in elite apnea divers. Int J Sports Med 24(2):104-107

\section{EP3 O \\ Physiological profile evaluation of indoor skydiving}

\author{
E. Limonta, S. Rampichini, S. Shokohyar, E. Cè, S. Longo, \\ B. Coratella, A. Montaruli, F. Esposito
}

Department of Biomedical Sciences for Health, University of Milan, Milan, Italy

Purpose: Indoor skydiving is an emerging sport derived from outdoor skydiving. It takes place within vertical wind tunnels that allow to move floating by means of powerful air flows. Recognized by the International Aeronautical Federation (FAI), it currently provides 6 disciplines. Despite its increasing popularity, very little is known about the physiological profile of this discipline yet. On this basis, we evaluated the cardiorespiratory, metabolic and perceptive response to some different postures that were adopted in the wind tunnel.

Methods: Ten subjects (age $32 \pm 2$ years; body mass $74 \pm 4 \mathrm{~kg}$; stature $173 \pm 3 \mathrm{~cm}$ ), skydiving instructors, volunteered to the study. Inside the tunnel, the main cardiorespiratory parameters were monitored during the four static positions maintained for $4 \mathrm{~min}$, in a random order: supine, prone, sitting, inverted. At the end of each position, a blood sample was collected to measure lactate concentration. Moreover, the subjective perception of effort (RPE) has been evaluated by Borg scale. Outside the tunnel, the same evaluations were carried out during the supine and sitting position maintained for $4 \mathrm{~min}$. The level of significance was set at $\alpha<0.05$.

Results: Inside the tunnel, cardiorespiratory variables values were significantly higher $(p<0.05)$ in inverted position than in the others. Also general, muscular, respiratory RPE and blood lactate concentration showed higher values $(p<0.05)$ in inverted position. Moreover, supine and sitting position inside the tunnel elicited higher values $(p<0.05)$ of respiratory frequency $\left(f_{\mathrm{R}}\right)$, VE and heart rate $\left(f_{\mathrm{H}}\right)$ than the same posture outside the tunnel.

Conclusions: This is the first study that analyzed the physiological response to indoor skydiving. Our results indicate that indoor skydiving is a discipline with a mainly aerobic commitment and that significant metabolic differences can be observed depending on the posture taken in the tunnel. However, the same posture (supine or sitting) inside the tunnel is metabolically more demanding than outside. These preliminary results will be implemented by further assessments of the metabolic commitment and muscular involvement both in static positions and during dynamic sequences as in official competitions, in order to provide instructor with useful information for training.

\section{EP3 O \\ Moving from 2000 to $3500 \mathrm{~m}$ of simulated altitude: physiological responses with different intensities of effort}

\author{
A. Savoldelli, A. Fornasiero, S. Skafidas, G. Boccia, \\ F. Stella, C. Zoppirolli, L. Mourot, L. Bortolan, F. Schena, \\ G. Vernillo, B. Pellegrini
}

CeRiSM (Research Centre of Mountain Sport and Health), University of Verona, Rovereto, Italy

Purpose: There are competitions characterized by starting from an altitude to finish at a higher one, in progressive hypoxia. We investigated the physiological responses during a 1-h exposure to progressive hypoxia $(\mathrm{PH})$ as well as the effects of different intensities of effort randomly imposed to the subjects during the interventions. Methods: 11 subjects underwent a maximal ramp test to obtain the peak power output $(\mathrm{PPO})$ both in normoxia $(\mathrm{N})$ and in normobaric hypoxia $\left(\mathrm{H}, \mathrm{FiO}_{2} 13.4 \%\right)$. After that they randomly completed 3 different 1-hour sessions under the same $\mathrm{PH}$ stimulus $\left(\mathrm{FiO}_{2}\right.$ starting from 16.25 to $13.4 \%$, simulating from 2000 to $3500 \mathrm{~m}$ ) at different intensities: no effort (H_NoPO), 50\% of the PPO in $\mathrm{H}(\mathrm{HH})$ and $50 \%$ of the PPO in N (HN). During the 1-h we monitored continuously pulse oxygen saturation $\left(\mathrm{SpO}_{2}\right)$, oxygen consumption, $\mathrm{RPE}$ and in 4 different time points the cerebral blood flow (CBF). Neuromuscular function was assessed pre and post the 1-h test.

Results: PPO and $\mathrm{VO}_{2 \max }$ were significantly lower at the end of the ramp test in $\mathrm{H}(P<0.001,-14.2$ and -20.6 , respectively). An effect of time was found on $\mathrm{CBF}$ (increase) and $\mathrm{SpO}_{2}$ (decrease) during the 3 conditions. $\mathrm{SpO}_{2}$ during $\mathrm{HH}$ and $\mathrm{HN}$ decreased with a higher rate when compared to H_NoPO. Even if subjects reported an augmented RPE across $\mathrm{HH}$ and $\mathrm{HN}$, no increase in oxygen consumption was found during the 1 -h. MVC decreased post $1 \mathrm{~h}$ cycling in $\mathrm{HH}$ and $\mathrm{HN}(P<0.01,-9.1$ and -10.9 , respectively $)$ while no 
effects after H_NoPO. Maximal voluntary activation (VA) was reduced after the 3 different interventions $(P<0.05)$.

Conclusions: Ramp test results are in line with literature [1]. During an effort in $\mathrm{PH}$ oxygen consumption does not change both during $\mathrm{HH}$ and $\mathrm{HN}$, confirming the idea of a maintained efficiency at simulated altitude [2]. The general reduction in $\mathrm{SpO} 2$ during the three 1-hour sessions may be by itself the cause of the reduction in VA, as an index of central fatigue.

\section{References}

1. Wehrlin JP, Hallen J (2006) Linear decrease in VO2max and performance with increasing altitude in endurance athletes. Eur $\mathrm{J}$ Appl Physiol 96:404-412

2. Noordhof DA et al (2013) Is gross efficiency lower at acute simulated altitude than at sea level? Int J Sports Physiol Perform $8: 319-322$

\section{EP3 O}

Heart rate variability decrease after strenuous exercise: similar response in type 1 diabetes and healthy subjects

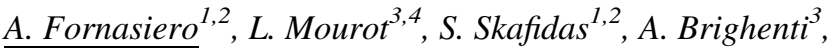 \\ $\overline{\text { A. Gentilin }^{2}, \text { F. Stella }}{ }^{1,2}$, A. Zignoli $^{1}$, A. Savoldelli, ${ }^{1,2}$, \\ B. Pellegrini ${ }^{1,2}$, C. Tarperi $^{2}$, F. Schena ${ }^{1,2}$ \\ ${ }^{1}$ CeRiSM, Sport Mountain and Health Research Centre, University \\ of Verona, Rovereto, Italy; \\ ${ }^{2}$ Department of Neurosciences, Biomedicine and Movement Sciences, \\ University of Verona, Verona, Italy; \\ ${ }^{3}$ Laboratory of Prognostic Markers and Regulatory Factors \\ of Cardiovascular Diseases and Exercise Performance, Health, \\ Innovation Platform (EA 3920), University of Bourgogne Franche- \\ Comté, Besançon, France; \\ ${ }^{4}$ Tomsk Polytechnic University, Tomsk, Russia
}

Purpose: Reduced cardiac parasympathetic activity and depressed heart rate variability (HRV) have been reported in Type 1 diabetes (T1D) (Dafaalla et al. 2016). Exercise is a non-pharmacological therapeutic tool recommended by diabetes treatment guidelines (Colberg et al. 2016). Spontaneous exercise could be of high addedvalue for patients but the effects of a single bout of strenuous exercise is not well described in T1D. The aim of this study was to evaluate the effect of an acute and strenuous exercise on HRV in T1D patients.

Methods: Nine T1D patients (T1D; age $39.0 \pm 11.1$ years, BMI $\left.23.1 \pm 2.4 \mathrm{~kg} / \mathrm{m}^{2}\right)$ and 11 healthy subjects $(\mathrm{H} ; \quad$ age $37.2 \pm 15.4$ years, BMI $22.7 \pm 2.2 \mathrm{~kg} / \mathrm{m}^{2}$ ), involved in the Run for Science project (Lippi and Schena 2017), were evaluated before (PRE) and after (POST) a 21-km run. HRV was assessed in supine position after $10 \mathrm{~min}$ of rest through 5-min of RR intervals recording (Polar RS800, Finland). A two-way (time $\times$ group) RM ANOVA was performed to assess the effects of run and disease on HRV indices.

Results: Exercise duration was similar in the two groups $(110.0 \pm 18.7$ vs $104.0 \pm 13.2 \mathrm{~min}$ in T1D and $\mathrm{H}$ respectively). A significant effect of time $(p<0.001)$ was reported on HRV indices with no significant time $\mathrm{x}$ group interaction $(\mathrm{p}>0.05)$. Lower mean RR was reported in POST compared to PRE, without differences between groups $(749 \pm 110$ vs $1156 \pm 196 \mathrm{~ms}$ and $825 \pm 141$ vs $1166 \pm 162 \mathrm{~ms}$ in T1D and $\mathrm{H}$ respectively). Parasympathetic activity indices, such as the root-mean-square of successive RR interval difference (Ln-RMSSD) was reduced in POST compared to PRE, without differences between groups $(2.3 \pm 0.5$ vs $3.9 \pm 0.9 \mathrm{~ms}$ and $2.7 \pm 0.9$ vs $3.9 \pm 0.7 \mathrm{~ms}$, in T1D and $\mathrm{H}$ respectively).
Conclusions: In well-controlled and trained T1D patients a single bout of strenuous exercise induced similar changes in HRV indices compared to healthy subjects.

\section{References}

Dafaalla MD, Nimir MN, Mohammed MI, Ali OA, Hussein A (2016)

Risk factors of diabetic cardiac autonomic neuropathy in patients with type 1 diabetes mellitus: a meta-analysis. Open Heart 3

Colberg SR, Sigal RJ, Yardley JE, Riddell MC, Dunstan DW, Dempsey PC et al (2016) Physical activity/exercise and diabetes: a position statement of the American Diabetes Association. Diabetes Care 39:2065-2079

Lippi G, Schena F (2017) Run for Science (R4S): the history of a successful project of precision and laboratory medicine in sport and exercise. J Lab Precis Med 2(4)

\section{TP4 K}

\section{The rate of torque development scaling factor is helpful to identify inter-limb asymmetry in quadriceps and hamstring of soccer players}

\author{
G. Boccia $^{1,2}$, P. R. Brustio ${ }^{1}$, G. Buttacchio ${ }^{2}$, \\ M. Calabrese $^{2}$, R. Casale $^{2}$, A. Rainoldi ${ }^{1}$
}

${ }^{1}$ NeuroMuscularFunction | Research Group, School of Exercise and Sport Sciences, Department of Medical Sciences, University of Turin, Turin, Italy;

${ }^{2}$ Habilita Care and Research Rehabilitation Unit, Zingonia, Bergamo, Italy

Purpose: It seems that the lower limbs of soccer players are characterized by significant compound muscle strength asymmetries. The peak torque exerted during fast concentric contractions is widely adopted to identify these asymmetries. In this study we compared inter-limb asymmetry obtained in concentric contractions with maximal voluntary isometric contraction (MVIC), maximal rate of torque development (RTD) and the RTD scaling factor (RTD-SF). RTD-SF quantifies the extent to which RTD scales with the intensity of the contraction. The slope of the linear regression that exist between the peak torque and the peak RTD obtained, for each pulse, in a series of fast force pulses performed across a range of submaximal values.

Methods: Quadriceps and hamstring strength was evaluated in both limbs of 22 elite young soccer players (males, $17 \pm 1$ years). Seated on an ergometer, participants performed to MVICs, three maximal concentric isokinetic contractions at $240 \%$ s (CONC) and the RFD-SF protocol. The interlimb difference was calculated for each parameter and the percentage of athletes that presented an interlimb difference higher than $10 \%$ was identified.

Results: The average inter-limb difference in MVIC torque was comparable for quadriceps and hamstring $(9.9 \pm 8.0 \%$ and $10.1 \pm 6.2 \%$, respectively). For all other indices (maximal RTD, CONC, and RTD-SF) hamstrings showed on average greater inter-limb asymmetry than quadriceps. The RTD-SF (for both muscle groups) and maximal RTD (only for quadriceps) were the indices that presented the highest inter-limb asymmetry, on average $\approx 17 \%$. More than $60 \%$ of players presented an inter-limb asymmetry higher than $10 \%$ in RTD-SF. Conclusions: The RTD-SF identified more players with a relevant inter-limb asymmetry both in quadriceps and hamstring. RTD-SF was particularly compromised in the knee flexors of non-dominant limb. If this imbalance may be seen a risk factor for musculoskeletal injury is unknown. However, since RTD-SF provides meaningful information about the quickness of producing force across a wide rage of 
contraction intensities, we advance that future study should investigate if RTD-SF imbalance may be an injury risk.

\section{TRAINING, PERFORMANCE AND EVA- LUATION METHODS 4}

\section{TP4 O \\ Evidence of balance training-induced improvement in soccer-specific skills in U11 soccer players}

\author{
G. Coratella $^{1}$, E. Cè ${ }^{1}$, S. Longo ${ }^{1}$, E. Paleari $^{1}$, A. Riboli $^{1}$, \\ E. Limonta $^{1}$, E. Roveda, S. Rampichini ${ }^{1}$, F. Esposito ${ }^{1}$ \\ ${ }^{I}$ Department of Biomedical Sciences for Health (SCIBIS), Università \\ degli Studi di Milano, Milan, Italy
}

Purpose: The present study aimed to determine the role of balance training in improving technical soccer skills in young players.

Methods: Two U11 soccer teams were randomly assigned one to either balance training (BT; $n=22)$ or control group $(\mathrm{Ctrl} ; \mathrm{n}=21)$. At the end of their habitual soccer training (identical in BT and Ctrl), BT underwent additional balance training for 12 weeks ( 3 sessions/ week, 20 min per session), while Ctrl had a 20-min scrimmage. Before and after the intervention, BT and Ctrl underwent two soccerspecific tests (Loughborough Soccer Passing, LSPT, and Shooting, LSST, Tests), and bipedal and unipedal balance evaluations.

Results: After intervention, both groups decreased the trials time and improved passing accuracy, with larger improvements in BT than Ctrl [LSPT penalty time $\left(\mathrm{CI}_{95} \%\right)$ : $2.20 \mathrm{~s}(-2.72 /-1.68)$; $\mathrm{ES}\left(\mathrm{CI}_{95} \%\right)$ : $-2.54 \mathrm{~s}(-3.34 /-1.74)$ ]. Both groups improved balance ability, with BT showing larger increments in bipedal tests than Ctrl [static balance: $-29 \mathrm{~mm}(-42 /-16)$; ES: -1.39 (- 2.05/- 0.72); limit of stability: $4 \%$ (3/5); ES 3.93 (2.90/4.95); unipedal quasi-dynamic balance: 0.07 a.u. (0.03/0.11); ES: $1.04(0.40 / 1.67)$ and active range of motion: $-5 \%(-8 /-2)$; ES $-0.89(-1.51 /-0.26)]$. Low-tomoderate correlations between the players' technical level and unipedal balance ability were retrieved, particularly in the non-dominant limb ( $R$ from 0.30 to 0.48 ).

Conclusions: Balance training improved some technical soccer skills more than habitual soccer training alone, suggesting that young soccer players may benefit from additional balance training added to their traditional training.

\section{References}

Rössler R, Junge A, Bizzini M, Verhagen E, Chomiak J, Aus der Fünten K, Meyer T, Dvorak J, Lichtenstein E, Beaudouin F, Faude O (2017) A multinational cluster randomised controlled trial to assess the efficacy of ' 11 + Kids': a warm-up programme to prevent injuries in children's football. Sport Med, 1-12

Keywords: Football; Stability; Unipedal stance test

\section{TP4 O}

Comparison between iso-inertial eccentric and plyometric training effects in young soccer players

\author{
A. Buonsenso ${ }^{1}$, G. Fiorilli ${ }^{1}$, L. Catalano ${ }^{2}$, M. Centorbi ${ }^{2}$, \\ $\overline{\text { E. Iuliano }^{3}, \text { A. di Cagno }}{ }^{2}$, G. Calcagno ${ }^{1}$ \\ ${ }^{1}$ Department of Medicine and Health Science, University of Molise, \\ Italy; \\ ${ }^{2}$ Department of Movement Human and Health Sciences, Italy; \\ ${ }^{3}$ Faculty of Psychology of University eCampus, Italy
}

Purpose: To examine the effects of 8 weeks of Iso-Inertial eccentric training compared with plyometric training in young soccer players. Methods: 36 young male players $(15 \pm 0.6$ years $)$ were randomly assigned to Iso-Inertial Training group (IIT, $\mathrm{n}=12$ ), Plyometric Training group $(\mathrm{PT}, \mathrm{n}=12)$ and Control group $(\mathrm{C}, \mathrm{n}=12)$, the three group trained two times a week. IIT consisted of 2 sets of 6-10 repetitions in 3 specific overloaded exercises using the iso-inertial Flycon-Power (Cuneo-Italy) machine. PT used a comparable volume (2 sets of 6-10 reps), in 2 vertical and 1 horizontal plyometric exercises. $\mathrm{C}$ performed the usual training program. Pre and postintervention tests included Squat Jump, Drop Jump and Hopping Test using Optojump (Microgate-Bergamo, Italy).

Results Group comparison showed significant differences in the Drop Jump flight time, between Iso-Inertial and Plyometric Training $(\mathrm{p}=0.026)$, Iso-Inertial and control group $(\mathrm{p}=0.004)$, and Hopping Test flight time between Iso-Inertial and Control group $(\mathrm{p}=0.027)$. Significant effects within subjects were found $\left(F_{(2 ; 10)} 4.241\right.$; $p=0.05$ ).

Conclusions: An Iso-Inertial training program represents a promising tool and a novel exercise paradigm to enhance explosive strength in young soccer players. Further investigations may assess improvement in specific soccer performance as changes of direction and horizontal component of strength.

\section{References}

Tous-Fajardo J, Gonzalo-Skok O, Arjol-Serrano JL, Tesch P (2016) Enhancing change-of-direction speed in soccer players by functional inertial eccentric overload and vibration training. Int J Sports Physiol Perform 11(1):66-73

de Villarreal ES, Suarez-Arrones L, Requena B, Haff GG and Ferrete C (2015) Effects of plyometric and sprint training on physical and technical skill performance in adolescent soccer players. J Strength Cond Res 29(7):1894-1903 


\section{TP4 O}

Game-based vs open-skill mulitaleral training: effects of a 12-week program on motor skill acquisition and physical fitness development in soccer school children

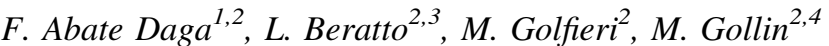

${ }^{I}$ Medical Physiopathology, Department of Medical Sciences, University of Turin, Italy;

${ }^{2}$ Adapted Training and Performance Laboratory, University School of Motor and Sport Sciences, University of Turin, Turin, Italy;

${ }^{3}$ Department of Clinical and Biological Sciences, University of Turin, Italy;

${ }^{4}$ Department of Clinical and Biological Sciences; University of Turin, Italy

Purpose: The importance of motor skill acquisition, physical development and health-related fitness of children has been significantly noted (William et al. 2008). Nowadays, sport disciplines are extremely focused in young athlete development to improve performance levels and to identify future talent (Huijgen et al. 2009). This study investigates the effects of a 12-week-game-based training on motor skills acquisition compared with a traditional open-skill multilateral schedule.

Methods: 31 children $(8 \pm 1$ years, $29 \pm 4 \mathrm{~kg}, 134 \pm 5 \mathrm{~cm}, 16 \pm 2$ of BMI, $2 \pm 1$ years of practice) belonging to a professional soccer academy were recruited for this study. All their parents approved the attendance at this study by signing an informed consent. Children were randomly arranged into one of the two experimental groups, trained for 12 weeks and tested on shuttle dribble test and $10 \times 5$ shuttle test using a pair of photocells (Witty, Microgate, Bolzano, Italy).

Results: Both Game-Based Group (G-BG) and Open-Skill Group (OSG) improved significantly in shuttle dribble test (G-BG $-19 \%$, $\mathrm{p}=0.001 ; \mathrm{O}-\mathrm{SG}-14 \%, \mathrm{p}=0.0037)$ and shuttle run test $(\mathrm{G}-\mathrm{BG}$ $-6 \%, p=0.0021 ; \mathrm{O}-\mathrm{SG}-4 \% \mathrm{p}=0.0047)$ No difference was found between the groups at the end of the study.

Conclusion: Data shows that both game-based training and an openskill multilateral schedule can provide improvements in children motor skills acquisition and fitness development. Considering this, it might be possible to advice trainers to focus on both technical and fitness abilities to ensure a complete coordinative and physical development.

\section{References}

Huijgen BCH, Elferink-Gemser MT, Post WJ, Visscher C (2009) Soccer skill development in professionals. Int $\mathrm{J}$ Sports Med 30:585-591

Williams HG, Pfeiffer KA, O'Neill JR et al (2008) Motor skill performance and physical activity in preschool children. Obesity 16(6):1421-1426

\section{TP4 O \\ Developing a EMG-based biofeedback to teach the proper activation of scapular stabilizers during upper limb exercises}

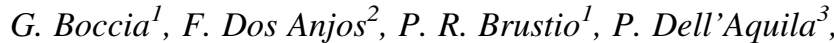 \\ M. Gazzoni ${ }^{2}$, A. Rainoldi ${ }^{1}$ \\ ${ }^{1}$ NeuroMuscularFunction | Research Group, School of Exercise \\ and Sport Sciences, Department of Medical Sciences, University \\ of Turin, Turin, Italy; \\ ${ }^{2}$ LISiN, Politecnico di Torino, Torino, Italy; \\ ${ }^{3}$ SUISM, University of Turin, Turin, Italy
}

Purpose: Biofeedback based on electromyograms (EMGs) can be used to provide real-time feedback on the muscle activation during the execution of an exercise. This can be important when novice individuals are learning new exercises in order to assist them in discriminating a correct from an incorrect pattern of muscle activation during exercise. Indeed, inexperienced individuals typically do not have precise awareness of correct muscle activation and movement execution.

In this project, which is part of the funding program POR FESR, we are developing an EMG based biofeedback running on a smartphone application in order to provide information about the correct activation of stabilizer muscles during upper limb exercises. The consortium is constituted by research groups of the University of Turin and Politecnico di Torino and by the company OT Bioelettronica.

To do that we (1) identified the proper electrode positioning on relevant muscles, (2) developed a smartphone-based application, and (3) will test the efficacy and usability of the biofeedback.

Methods:

Aim 1 . To identify the optimal electrode positioning, 16 young male volunteers, well accustomed to strength training exercises, were recruited. They were instructed to perform five repetitions of five different exercises (lat pull down, seated rows, bent over row, onearm dumbbell rows, low row with suspension straps), both in a correct (scapular stabilizer activated) and incorrect (no activation of scapular stabilizers) way. We firstly recorded monopolar surface EMGs from the scapular stabilizers (medial and lower portions of trapezius muscle) and from the primal movers (latissimus dorsi), using with three grids of 64 surface electrodes $(16 \times 4$ electrodes for trapezius and $8 \times 8$ for latissimus dorsi). Then, we processed the raw monopolar signals to simulate conventional bipolar detection system with $2 \mathrm{~cm}$ inter-electrode distance and electrode surface of approximately $1 \mathrm{~cm}^{2}$.

Results:

Aim 1. The optimal electrode positioning was identified as the bipolar detection configuration that maximized the detectable differences between correct and incorrect executions.

Aim 2. The smartphone application has been produced by the company.

Aim 3. Should be accomplished in the next stage of the project.

Discussion: This project will provide an innovative and user-friendly interface based on EMG biofeedback developed to help strength coach and clinicians when teaching a number of upper limb exercises. The device is currently undergoing validation and reliability studies. 


\section{PHYSICAL EXERCISE AS PREVENTION AND THERAPY 3}

\section{EH3 K \\ Adapted physical exercise for people with chronic disease: the first "Palestra Salute" in the Piedmont Region}

\author{
A. Rainoldi ${ }^{1}$, P.R. Brustio ${ }^{1}$, A. Mulasso ${ }^{1}$ \\ ${ }^{I}$ NeuroMuscularFunction | research group, School of Exercise \\ and Sport Sciences, Department of Medical Sciences, University \\ of Turin, Turin, Italy
}

Purpose: a recent law proposal $\left(n^{\circ} 231\right.$ presented by the President of Piedmont Regional Council) underlined the need for radical changes in lifestyle and for tailored physical exercise (PE) in persons with chronic diseases sensitive to PE. To answer to these needs, the first "Palestra Salute" (PS) of the Piedmont Region has been realized. The aim of this study is to describe theoretical, methodological and instrumental issues related to the realization of the PS.

Methods: PE is addressed firstly to people affected by musculoskeletal disorders and metabolic diseases who have been sent to the PS by the general medical doctors. PE was structured on the basis of scientific evidence (Ageberg et al. 2010; Wiesmeier et al. 2017) in three areas of intervention: (a) cardiorespiratory, (ii) neuromuscular, (iii) core stability and balance. PE was performed twice a week for 16 weeks, in groups of $12-15$ persons, supervised by 4-5 experts with a Master Degree in Adapted Physical Activities. The fraction of work for each area and the type of exercises were adapted for: (i) chronic disease, (ii) individual level of physical functioning established on the basis of baseline assessment. The assessment is multidimensional using validated tools to detect general health status and physical, cognitive, psychosocial functioning. In addition to baseline evaluation, intermediate and final assessment sessions were organized. Guidelines with specifications of actions, times and goals for each population who can benefit from PE were designed and provided.

Conclusions: The program of PE (currently under way) seems to be well tolerated by individuals and in a short time it will be implemented in a wider sample of people chronically ill. If the data will confirm the effectiveness of the PE program, the intervention standards may be scaled-up and used to obtain the certification of "PS" throughout the regional territory.

References

Ageberg E, Link A, Roos EM (2010) Feasibility of neuromuscular training in patients with severe hip or knee OA: the individualized goal-based NEMEX-TJR training program. BMC Musculoskelet Disord 11:126

Wiesmeier IK, Dalin D, Wehrle A, Granacher U, Muehlbauer T, Dietterle J et al (2017) Balance training enhances vestibular function and reduces overactive proprioceptive feedback in elderly. Front Aging Neurosci 9

Acknowledgements: This work was supported by Fondazione CRT with the project "Esercizio fisico, consapevolezza e stili di vita".

\section{EH3 O \\ Back to sport after an ACL reconstruction: a reconditioning protocol for a safe return to play}

\author{
R. Purello D'Ambrosio, A. Vilet, S. Belot, C. Herbin, \\ R. Pionnier, J. Esnault, C. Gorny, L. De Maria, \\ M. Peyre
}

Département of Médecine Physique, Rééducation Orthopédique, Traumatologie du Sport des Hôpitaux de Saint-Maurice, Paris, France

Purpose: A specific rehabilitation protocol is essential for the management of the athlete after an ACL reconstruction; the principal goal of patients and therapists is to return to sport (RTS) as soon as possible, possibly performing at the same level as pre-injury and preventing a re-injury. Our protocol's aim is a safe return to sport (RTS) for our patients, working specifically on his strength, coordination and proprioception.

Methods: At 6th month after the surgery, the knee has typically a full range of movement, no effusion after exercises and an adequate stability and proprioception, the graft is considered totally healed and the patient can start working on sport-specific skills, still avoiding contact and full pivots until the 8th month.

In this phase, we've been evaluating strength, coordination and static and dynamic stability of our patients using respectively: isokinetic strength test, low quarter y balance test (LQYBT), single and double leg hop test, dynamic valgus control by force platform gait analysis.

Poor results at the dynamic tests and a strength deficit $>30 \%$ are highly predictive of a re-injury and have been the criteria for including athletes in a reconditioning protocol of 5 weeks during which the patient has been working on a neuromuscular training and reconditioning protocol with physiotherapists, hydrotherapists, psychologists, MDs and physical trainers. The same tests used at the beginning of the 5 weeks, have been performed during and at the end of the protocol.

Results: Results showed improvements in all the functional test and a reduction of strength imbalance, allowing the patient to a safe RTS, with a low re-injury risk.

Keywords: ACL, reconstruction, graft, isokinetic test, Y balance test, neuromuscular training, reconditioning, rehabilitation, injury, athlete, sport

\section{References}

Chen C-H (2009) Graft healing in anterior cruciate ligament reconstruction.

https://www.ncbi.nlm.nih.gov/pmc/articles/PMC2757018/

Cvjetkovic DD, Bijeljac S, Palija S, Talic G, Radulovic TN, Kosanovic MG, Manojlovic S (2015) Isokinetic testing in evaluation rehabilitation outcome after ACL reconstruction. https://www.ncbi.nlm.nih.gov/pmc/articles/PMC4384850/

Nyland J, MacKinlay KGW, Wera J, Krupp RJ (2015) Return to play decision-making following anterior cruciate ligament reconstruction: multi-factor considerations, chapter 120. In: Doral MN, Karlsson J (eds) Sports injuries prevention, diagnosis, treatment and rehabilitation, 2nd edn. Springer, Berlin, pp 1491-1502

Paterno MV. Incidence and predictors of second anterior cruciate ligament injury after primary reconstruction and return to sport. https://www.ncbi.nlm.nih.gov/pmc/articles/PMC4641548/

Thomeé R, Kaplan Y, Kvist J, Myklebust G, Risberg MA, Theisen D, Tsepis E, Werner S, Wondrasch B, Witvrouw E (2011) Muscle strength and hop performance criteria prior to return to sports after ACL reconstruction. https://link.springer.com/article/10.1007/ s00167-011-1669-8 


\section{$60 \mathrm{EH} 3 \mathrm{O}$}

Effects of three different protocols on physical, psychological and cognitive area in Multiple Sclerosis patients: a pilot study

\author{
E. Grazioli ${ }^{1}$, G. Borriello ${ }^{2}$, G. Chiodi ${ }^{3}$, E. Tranchita ${ }^{1}$, \\ C. Minganti $^{1}$, C. Cerulli ${ }^{1}$, A. Parisi ${ }^{1}$, F. Pigozzi $i^{1}$
}

${ }^{1}$ Sport medicine Unit, Department of Movement, Human and Health Sciences, University of Rome "Foro Italico";

${ }^{2}$ S. Andrea Hospital, University of Rome La Sapienza, Rome, Italy;

${ }^{3}$ Tor di Quinto Sports Center, Italian National Police, Rome, Italy

Purpose: Multiple sclerosis (MS) is a chronic progressive disease of central nervous system (CNS). It is characterised by brain and spinal cord lesions and plaques, which affect the myelin sheath, causing the inhibition of axonal transmissions. This disease causes a wide range of potential symptoms related to different areas. Commonly patients suffering from MS, were removed from any physical effort. Recent literature has increasingly corroborated the hypothesis that a specific well-structured motor protocol may produce beneficial effects in these patients. The aim of this pilot study is to evaluate the effects of three different protocols of intervention on psychological, cognitive functions and walking ability in patients affected by Multiple Sclerosis and consequently their Quality of Life (QOL).

Methods: This randomized controlled trials, after the approval of Ethical Committee (RIF.CE 4520), evaluated several parameters (BMI, strength, balance, fatigue etc.) in 45 SM patients (EDSS 2.5-6.5) to assess a non-conventional training (WeSET), focused on balance and proprioceptive exercise to rebuild the walking ability; a combined training (CT) composed by endurance and a strength phases; and a physiokinetic therapy (FKT).

Results: Our preliminary results reported that both WeSET and CT protocols provide positive effects on balance and walking ability in MS patients. A general increase on QOL was reported in all three groups. These data suggest that the combination of different protocols focused on the improvement of strength, balance, self-esteem, and also of general motor skills is needed to maintain the autonomy and a high quality of life through the disease. A multidisciplinary approach could be a new effective strategy for MS patients.

References

Milo R, Kahana E (2010) Multiple sclerosis: geoepidemiology, genetics and the environment. Autoimmun Rev

Pearson M, Dieberg G, Smart N (2015) Exercise as a therapy for improvement of walking ability in adults with multiple sclerosis: a meta-analysis. 2015 by the American Congress of Rehabilitation Medicine

Motl RW, Gappmaier E, Nelson K, Benedict RH (2011) Physical activity and cognitive function in multiple sclerosis. J Sport Exerc Psychol 33(5):734-741

Keywords: Multiple sclerosis, training, quality of life, balance

\section{EH3 O}

The role of exercise and of the expert of the movement at the end of the rehabilitation process

for the maintenance of the best autonomy and quality of life: a practical case: physical activity adapted to a person with right hemiplegia from hemorrhagic stroke; effectiveness analysis and perspective vision

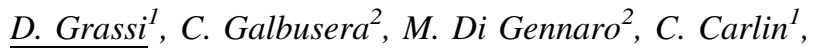 \\ R. Pazzona ${ }^{3}$, M. Quarantelli ${ }^{1}$ \\ ${ }^{1}$ San Raffaele University Rome; \\ ${ }^{2}$ Fisioterapia Alessandria; \\ ${ }^{3}$ University of Cagliari
}

Purpose: The aim was to gain further progress in gait in a person affected by hemorrhagic stroke 6 years before, with stabilized outcomes of right-hemiplegia and aphasia. The activity is not part of a rehabilitation therapy.

Methods: The gait analysis identified the pathological deviations. The person's health was assessed with questionnaires and physical tests before and at the end of a 6-month period: The Short Form (36) Health Survey; Edinburgh Handedness Inventory; 6 Minutes Walking Test and the Rate of Perceived Exertion (RTE); $1 \mathrm{~km}$ walking test using a GPS tracker; Trunk Impairment Scale Italian version (TIS-I); Berg Balance Scale; Modified Barthel Index. A program of adapted physical activity was designed and delivered.

Result: Improvement of the gait speed, better tone of the trunk muscles, distal control, autonomy and perceived well-being were registered. The gait cycle is still pathological but a better motor control results in a more symmetrical length and duration of the two steps. Indicators for the pathological gait analysis are not still available and the use of physiological diagrams is not an accurate system. Indicators deriving from the bio-psycho-social model are suitable for rehabilitative cares but not effective to design a physical training. A person suffering from neurological damage requires an appropriate approach to his/her specific needs, confirming the centrality of nervous system training. Further data and studies are required.

References

Saraceni VM, Fletzer DA, Esercizio in medicina riabilitativa, Piccin ISBN 978-88-299-1976-5, edizione anno 200

Davies PM, Step by step. Springer 2001, ISBN 978-88-470-0128-2

Perry J, Gait analysis. Normal and pathological function, ISBN 978-1-55642-192-1

Cimolin V, Galli M (2014) Summary measures for clinical gait analysis: a literature review. Gait Posture 39(4):1005-1010. https://doi.org/10.1016/j.gaitpost.2014.02.001 


\section{$62 \mathrm{EH} 30$}

Effect of exercise on knee flexibility and strength in a subject with Arthrogryposis multiplex congenital: a case study

\author{
G. Calcagno $^{1}$, E. Iuliano $^{2}$, F. Vinciguerra $^{1}$, A. Buonsenso $^{1}$, \\ G. Fiorilli ${ }^{1}$, A. di Cagno ${ }^{3}$
}

${ }^{1}$ Department of Medicine and Health Sciences, University of Molise, Italy;

${ }^{2}$ Faculty of Psychology, eCampus University, Italy;

${ }^{3}$ Department of Movement, Human and Health Sciences, University of Rome "Foro Italico", Italy

Purpose: Arthrogryposis multiplex congenital (AMC) is a disease characterized by a congenital joint contracture in two or more areas of the body. The aim of the study was to evaluate the effects of a protocol of physical exercise, performed both in water and on dry land, in improving knee flexibility and strength in a subject with AMC. The participant was a 35 years old female (weight $=55 \mathrm{~kg}$; height $=155 \mathrm{~cm})$ that had important limitations in leg flexion and extension.

Methods: The participant performed 3 months of training, 6 months of inactivity, and again 3 months of training. At the beginning and at the end of the two training periods, 2 variables were measured that were the range of motion (ROM) of knee, and the strength of the knee extension muscles. These two variables were respectively assessed by motion analysis and isometric dynamometry. This study design allowed to evaluate both the effects produced by training and inactivity.

Results: The results showed an increment of knee ROM of $6^{\circ}$ in both legs after the first training period. These benefits were lost after the 6 months of inactivity. Again, an increment of knee ROM was obtained after the second training period, with similar values to those obtained in the first training period. The isometric dynamometry showed an increment of $+8.5 \mathrm{~N}$ in left leg and $+7.5 \mathrm{~N}$ in right leg in knee extension strength after the second training period. The participant also reported a reduction of pain during the training periods, whereas an increment of pain was reported during the inactivity phase.

Conclusion: This case report showed that exercise can produce important benefits in subjects with AMC. The results suggest that it is essential to avoid inactivity period to maintain the benefit obtained by exercise. At the best of authors' knowledge, no previous study was performed to evaluate the benefits of exercise in subjects with AMC. Reference

Nouraei H et al (2017) Long-term functional and mobility outcomes for individuals with arthrogryposis multiplex congenita. Am J Med Genet A 173:1270-1278

\section{PHYSICAL EDUCATION AND SPORT PEDAGOGY 3}

\section{PE3 K}

The distribution of practice in learning a new movement in preteens

\author{
V. Perciavalle ${ }^{1}$, M. C. Petralia ${ }^{1,2}$, \\ M. S. Basile ${ }^{2}$, M. Coco $^{2}$ \\ ${ }^{1}$ Department of Educational Sciences, University of Catania; \\ ${ }^{2}$ Department of Biomedical and Biotechnological Sciences, \\ University of Catania
}

Purpose: The present study was carried out with the to evaluate if, and to what extent, in preteen children, different procedures of distribution in time of training sessions are capable to significantly influence the learning of a new movement.

Methods: About 1000 children of both sexes, aged between 6 and 10 years, were recruited for the study. The children were instructed to learn a movement of oculo-manual coordination, absolutely new to them, by using different modalities (concentrated or distributed) in the distributing of practice.

The children were grouped into three clusters (A, B and C) and each cluster was, in turn, subdivided into five sub-clusters, matching to the five classes of the elementary schools. The protocol contemplate a total of 120 total launches to be performed in a week, grouped as follows: group A carried out a training distributed over 6 consecutive days; group B, executed a training distributed in 3 alternating days (Monday, Wednesday, Friday), group C, performed a training distributed over 3 consecutive days (Monday, Tuesday, Wednesday). One week after the end of the learning phase each cluster repeated a session of 20 launches (two blocks of 10 separated by $5 \mathrm{~min}$ of recovery).

Results: The present study showed that, in terms of improvement, the learning of a new movement, performed using the concentrated practice appears less advantageous than that carried out with distributed practice.

However, what is learned with concentrated practice seems to last longer than that which is learned with the distributed practice. Furthermore, it has been seen that the speed of learning in males is greater than females.

\section{References}

Du Y, Valentini NC, Kim MJ, Whitall J, Clark JE (2017) Children and adults both learn motor sequences quickly, but do so differently. Front Psychol 8:158

Juliusa MS, Meira R, Shechter-Nissima Z, Adi-Japha E (2016) Children's ability to learn a motor skill is related to handwriting and reading proficiency. Learn Individ Differ 51:265-272

Keywords: Learning, Sport, Training sessions, Children 


\section{PE3 O}

\section{Identification and description of teaching styles in physical education: observation and analysis of teaching in middle school}

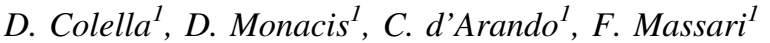 \\ ${ }^{1}$ Department of Clinical and Experimental Medicine, Laboratory of Didactic of Motor Activities, University of Foggia, Foggia, Italy}

Purpose: The motor competencies of the student are taught through the interaction of teaching styles (Mosston and Ashworth 2008) and the variability of the practice, to promote different ways of learning, personalized teaching interventions and to favor teaching mediation. The objective of the study is to identify, structure and experiment a list of behavioral teacher descriptors in the gym, to identify the teacher-pupil-pupil interaction modalities and the teaching styles used prevalently.

Methods: The sample consists of 6 teachers of physical education, three men (age $50 \pm 3.46$ ) and three women (age $53.33 \pm 3.51$ ). Six class groups were identified (two for each class of middle school, first, second and third). A list of descriptors of the teacher's behavior in the gym has been structured, corresponding to each style of teaching. Through systematic observation, the teaching-learning episodes proposed by each teacher in eight physical education classes, carried out with the same class group (Siedentop and Tannehill 2000), were detected. The style (or styles) of teaching used in $1 \mathrm{~h}$ of class through the predefined descriptors and the proposed learning episode was recorded. The purpose of the observation and the object of the survey were communicated to the teacher.

Results: In the present preliminary study, recurrent teacher behaviors were observed in the individual teaching-learning episodes, through the descriptors. It has emerged that every teacher, regardless of the class group, has mainly used styles of reproduction compared to those of production. In particular, learning episodes related to reproduction styles $(21.5 \pm 3.27)$ were compared to the production styles $(3.16 \pm 1.47)$.

Conclusions: The results obtained allow (a) to reconstruct the methods preferred by the teacher for proposing the motor tasks and organizing the activities in the gym, (b) the learning modalities of the students mainly solicited and (c) the modalities in which the teaching mediation is carried out. The analysis of the teaching is necessary to find out how the teacher interacts with the students and the ways of motor learning.

\section{References}

Mosston M, Ashworth S (2008) Teaching physical education, first on line edition

Siedentop D, Tannehill D (2000) Developing teaching skills in physical education. Mayfield Publ Company, Mountain View

\section{PE3 O}

The initial preparation of physical education teachers: trends, perspectives, problems. A critical review of the literature

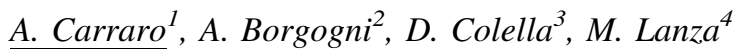

${ }^{1}$ Department of Biomedical Sciences, University of Padua, Italy;

${ }^{2}$ Department of Human Sciences, Society, and Health, University of Cassino and Southern Lazio, Italy;

${ }^{3}$ Department of clinical and experimental medicine, University of Foggia, Foggia, Italy;

${ }^{4}$ Department of Neuroscience, Biomedicine and Movement, University of Verona

Purpose: Quality Physical Education (PE) is strictly linked to the qualification of PE teachers. The phase of initial teacher education plays a key-role both for the acquisition of teaching skills and for the development of future teachers' behaviours, attitudes and value orientation. Quality PE teacher education (PETE) programmes are those where graduate teachers are lifelong learners who possess a deep knowledge of the subject area and a set of reflective, pedagogical and didactic skills and professional dispositions that allow them to design and deliver quality programmes for all students (AIESEP 2014). There is therefore a need to study the practices that occur in and across PETE programmes, to identify best practices as programmes strive to prepare effective teachers. To date, there is no comparative reference point on PETE programme practices.

Method: The search of the literature was conducted using online bibliographic databases (PubMed, PsychINFO, Cochrane Library, Education Source, and SPORTDiscus); 29 papers published in the last 20 years were identified and included in the review. Twenty studies regarded Europe, 4 Australia and New Zealand, 4 USA, 1 Canada, and 1 reported a trans-national perspective.

Results: We identified five study areas: pedagogies underlying initial PETE; methodological approach and contents of programmes; preservice teachers' views of their experience; attitudes and perceptions towards teaching students with disability or with special needs; cultural issues related to the recruitment and retention of ethnic minority teachers. Studies adopted diverse experimental designs, including qualitative methods (14 papers), mixed methods (2), quantitative approaches (6) and the critical discussion of pedagogies and curricola contents (7). Four studies regarded the provision of PE in primary education training courses.

Conclusions: Despite it is complex to identify common lines within different PETE programmes and to test their effectiveness, literature should be carefully considered to inform future actions aimed at recognising new paths for the preparation of new PE teachers in both primary and secondary school.

Reference

AIESEP (2014) AIESEP Position Statement on Physical Education Teacher Education 


\section{PE3 O}

\section{Peer victimization in primary school: gender difference}

\author{
C. Lucchetti $^{1}$, A. Nart ${ }^{1,2}$, V. Biancalana ${ }^{1}$ \\ ${ }^{1}$ University of Urbino, Department of Biomolecular Sciences, Urbino \\ Italy; \\ ${ }^{2}$ University of Padova, Department of Biomedical Sciences, Padova \\ Italy
}

Purpose: The peer victimization is frequent phenomenon in the school. The aim of study was to investigate the gender distinction in the peer victimization, also considering the role that physical activity inside and outside school could have in this phenomenon.

Methods: The study included 277 students (142 females; 134 males), between the ages of 8 and 10, attending the primary school. A questionnaire based on "Multi-dimensional Peer-Victimization Scale (MPVS)" (Mynard et al. 2000), Italian version (Scarpa et al. 2010) and adapted to the age of the subjects, was given, to evaluate four areas of victimization (physical victimization, verbal victimization, social manipulation and attack on property). In addition, subjects were asked which physical activities they practiced in and out of school.

Results: The results showed that the phenomenon victimization is present in a content way, and that males are more involved than females. Physical victimization falls more in the male gender (average value of $2 \pm 1.4$ and $1.6 \pm 0.9$ for the males and females, respectively), while the social manipulation in the women gender (average value of $1.9 \pm 1.2$ and $1.7 \pm 1.1$ for the females and males, respectively). The correlation between two or more macro-areas is there for the $74.32 \%$ of the cases and verbal victimization is the most widespread phenomenon in our sample $(9.46 \%)$. The extra-school physical activity is performed by the majority of students $(83.57 \%)$. Conclusions: The results confirm existing studies in the literature. The peer victimization includes not only being the obvious target of physical attacks but also includes more subtle forms of abuse, falling more in females behavior than in males.

\section{References}

Mynard H, Joseph S (2000) Development of the multidimensional peer-victimization scale. Aggress Behav 26:169-178

Scarpa S, Gobbi E, Paggiaro A, Carraro A (2010) Un contributo alla validazione italiana del Physical Self-Description Questiannaire Short. Giornale Italiano di Psicologia dello Sport 8:25-30

Scarpa S, Carrraro A, Gobbi E, Nart A (2012) Peer-victimization during physical education and enjoyment of physical activity. Percept Motor Skills 115:319-324

\section{PE3 O}

One-year evolution of gross motor coordination in children 8-11 years olds: a longitudinal study

\author{
V. Biino ${ }^{1}$, M. Giuriato, M. Lanza ${ }^{2}, F$. Schena ${ }^{2}$ \\ ${ }^{1}$ Department of Human Sciences, University of Verona; \\ ${ }^{2}$ Department of Neurosciences, Biomedicine and Movement Sciences, \\ University of Verona
}

Purpose: Studies that investigate the evolution of coordination during the children growth are relatively rare in the literature. Aims of the study are: (i) check how the coordination, measured with KTK (Vandorpe et al. 2009), changes with an increase of 1 year of age in children of 8-10 years; and (2) research if sports practice influences coordination.

Methods: Gross Motor Coordination (RS), weekly Physical Activity (PA) and sports practice have been measured twice in a sample of 137 children (70 females and 67 males); the first time when they were 8-9-10 years old (Pre) and the second time 1 year later (Post). The KTK test was used to measure the raw values (RS) of the children coordination. PA has been evaluated through the PAQ-C questionnaire (Gobbi et al. 2016). Finally, a question was asked to verify whether children practice some sports (yes/no).

Results: At overall Females and Males shows similar increase for RS $($ F-Pre $=204.2 \pm 27.6 ; \quad$ F-Post $=230.4 \pm 20.1 ; \quad$ M-Pre $=205.0 \pm$ 29.2; M-Post $=230.4 \pm 25.0)$ and, after 1 year they improve their performance $(\mathrm{p}<0.001)$.

In Pre, RS shows significant differences among the three groups of age $(8$-Pre $=190.6 \pm 27.0 ;$ 9-Pre $=207.0 \pm 26.3 ; \quad 10$-Pre $=216.8$ $\pm 26.4 ; \mathrm{p}<0.01)$ while this difference was not significant not in Post $\quad(8$-Post $=222.7 \pm 20.7 ; \quad 9$-Post $=233.7 \pm 23.4 ; \quad 10$-Post $=$ $225.9 \pm 21.7)$. RS POST, significantly improve for all groups ( $p<0.001)$. Considering the improvement for each age groups we measurement at 8 age $14.4 \%$; 9 age $11.4 \%$; 10 age $4.0 \%$

PA do not shows correlation with RS while the years of sport practice have a low correlation with RS $(\mathrm{r}=0.23$; $\mathrm{p}<0.01)$. As a whole Children who practice sport shows higher values of RS $(232.7 \pm 21.9)$ than non-practicing $(\mathrm{RS}=218.8 \pm 22.5 ; \mathrm{p}<0.05)$. One year variation of RS is similar in children practicing sport or not. Discussion: RS do not shows differences between female and males of 8-10 years old; it seems to increase with age, however, reducing the extent of its improvement. As could be expected, sports children show higher levels of coordination. 


\section{SATURDAY POSTER SESSION}

\section{TRAINING, PERFORMANCE AND EVALUATION METHODS}

\section{TP P \\ Relations between anthropometric parameters and jumping test in sporting grown-up subjects}

\section{A. Brunengo, P. Moise, F.A. Faure-Rolland}

\section{Università Studi di Torino, SUISM Torino}

Purpose: During the past years anthropometric parameters has been researched and used for expressing and evaluating the individual's state of shape. Recently has been highlighted how the Body Mass Index (BMI) presents a good correlations with the tests of state of physical form (vertical and long jumping tests) and it influences in a more or less significant way, according to the age and to the gender, the tests' results. Methods: In a group of male sporting subjects (n 26; age $23 \pm 2$ ) anthropometric data have been run. In particular it has been found the following characteristics: weight ( $\mathrm{w} 74.8 \mathrm{k} \pm 9.0 \mathrm{~kg}$ ) height (h $1.80 \pm 0.07 \mathrm{~m}$ ), reach test (RT $2.27 \pm 0.11 \mathrm{~m}$ ), Sargent test (SA $2.84 \pm 0.13 \mathrm{~m}$ ), Spike test two leg (SP $2.89 \pm 0.15 \mathrm{~m})$, Long jump test (LJ $2>25 \pm 0.26 \mathrm{~m}$ ).

Results: With the noticed data it has been extracted subjects' BMI (BMI $23 \pm 2 \mathrm{~kg} / \mathrm{m}^{2}$ ) and the elevation expressed in jumping tests based on Sargent test (ESA $0.58 \pm 0.08 \mathrm{~m}$ ) and on the Spike test (ESP $0.62 \pm 0.10 \mathrm{~m}$ ). The linear correlation analysis highlighted the following results between SA-SP $(0.983, \mathrm{p}<0.01)$, ESA-ESP $(0.966$, $\mathrm{p}<0.01)$, ESA-LJ (0.867; $\mathrm{p}<0.01)$, ESP-LJ $(0.885 ; \mathrm{p}<0.01)$. Between BMI and jumping tests the correlation values have been $<$ 0.373 ( $\mathrm{p}<0.1$ ). The best results of the tests of long jump (LJ) and of the vertical jump height (ESA-ESP) are expressed in a BMI $24 \div 25 \mathrm{~kg} / \mathrm{m}^{2}$. The distribution of the vertical jumping test (SA,SP) are worst for low BMI values. Collected data would seem being right with the observation of Lopes VP (2017).

Reference

Huang YC, Malina RM (2007) BMI and health-related physical fitness in Taiwanese youth 9-18 years. Med Sci Sport Exerc 39(4):701-708 Lopes VP, Cossio-Bolaños M, Gómez-Campos R et al (2017) Linear and nonlinear relationships between body mass index and physical fitness in Brazilian children and adolescents. Am J Hum Biol 29(6) Keywords: Anthropometric parameters, Body mass index, Jumping test, Vertical jump height

\section{TP P}

\section{Effects of a four-month judo training on gait speed and cadence in novice senior practitioners}

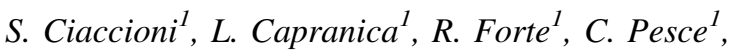 G. Condello ${ }^{1}$}

${ }^{1}$ Department of Movement, Human and Health Sciences, University of Rome Foro Italico

Purpose: To prevent falls and their consequences, effective programmes for older adults are needed. Average values and variability of gait parameters are used to quantify locomotion changes with aging associated with falls, and to monitor the effects of therapeutic interventions. The aim of this study was to investigate the effects of a 4-month judo training (1-h training session, twice a week) on gait performance in older individuals (age 60-76 years).

Methods: The experimental group (JG) included $16(\mathrm{~F}=8, \mathrm{M}=8$; $69.3 \pm 3.9$ years) participants to the judo programme, whereas the control group (CG) encompassed $14 \quad(\mathrm{~F}=5, \quad \mathrm{M}=9 ; \quad \mathrm{CG}$ $70.1 \pm 4.5$ years) moderately active controls (CG). Average values (AVG) and coefficients of variation $(\mathrm{CV})$ of speed $\left(\mathrm{m} \mathrm{s}^{-1}\right)$ and cadence (step $\mathrm{min}^{-1}$ ) were computed for habitual (HWS) and maximal walking speed (MWS) on a flat path (flat), on a narrow $(20 \mathrm{~cm}$ wide) corridor (corridor), and on a path with hurdles (hurdles). A 2 (gender) $\times 2$ ( group) $\times 2$ (time) ANOVA for repeated measures was applied to ascertain differences between groups in the different conditions $(p<0.05)$.

Results: A main effect emerged for Gender $(p=0.049)$ for speed, whilst Time $\times$ Group interactions were revealed for speed $(p=0.018)$ and cadence $(\mathrm{p}=0.001)$. For the Gender effect, the univariate analysis confirmed the differences, with women presenting lower AVG for

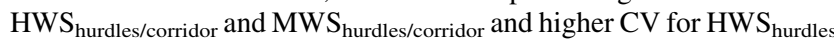
and $\mathrm{MWS}_{\text {hurdles }}$. Regarding the Time $\times$ Group interactions, significant increases were confirmed for AVG in JG for speed in $\mathrm{MWS}_{\text {hurdles }}$ (pre $1.53 \pm 0.17 \mathrm{~m} \mathrm{~s}^{-1}$, post $\left.1.64 \pm 0.19 \mathrm{~m} \mathrm{~s}^{-1}, \quad \mathrm{p}<0.001\right)$ and $\mathrm{MWS}_{\text {corridor }}$ (pre $1.67 \pm 0.19 \mathrm{~m} \mathrm{~s}^{-1}$, post $1.74 \pm 0.18 \mathrm{~m} \mathrm{~s}^{-1}$, $\mathrm{p}=0.047$ ) and for cadence in $\mathrm{MWS}_{\text {hurdles }}$ (pre 121.4 \pm 9.86 step $\min ^{-1}$, post $126.3 \pm 11.5$ step $\min ^{-1}, \mathrm{p}=0.005$ ) and $\mathrm{MWS}_{\text {corridor }}$ (pre $136.1 \pm 12.76$ step $\mathrm{min}^{-1}$, post $140.4 \pm 14.24 \mathrm{step} \mathrm{min}^{-1}$, $\mathrm{p}=0.026)$. Significant reductions were confirmed for AVG in CG for speed in $\mathrm{HWS}_{\text {corridor }}$ (pre $1.39 \pm 0.08 \mathrm{~m} \mathrm{~s}^{-1}$, post $1.28 \pm 0.17 \mathrm{~m} \mathrm{~s}^{-1}$, $\mathrm{p}=0.007$ ) and for cadence in $\mathrm{HWS}_{\text {corridor }}$ (pre $119.6 \pm 5.63$ step $\min ^{-1}$, post $112.8 \pm 9.01 \mathrm{step}^{\mathrm{min}}{ }^{-1}, \mathrm{p}=0.003$ ). Finally, significant reductions in $\mathrm{CV}$ were confirmed in JG for cadence in $\mathrm{HWS}_{\text {flat }}$ (pre $18.2 \pm 7.36 \%$, post $15.5 \pm 5.31 \%, p=0.007)$. The increases in AVG and reductions in $\mathrm{CV}$ indicate a positive effect of judo training on gait performance in novice senior judo population.

References

WHO (2018) WHO global action plan on physical activity 2018-2030 Whittle MW (2007) An introduction to gait analysis. Oxford Keywords: Gait analysis, Speed, Older persons, Judoka, Physical activity

\section{TP P}

Rectus abdominis activity during three sit up exercises with different range of motion

\author{
F. Luciano ${ }^{1}$, C. Zilianti ${ }^{2}$, L. Perini ${ }^{1}$, A. Guzzardella ${ }^{1}$, \\ G. Pavei $^{3}$
}

${ }^{I}$ Faculty of Medicine, University of Milan, Milano, Italy;

${ }^{2}$ International Medical School, University of Milan, Milano, Italy;

${ }^{3}$ Department of Pathophysiology and Transplantation, University of Milan, Milano, Italy

Purpose: When abdominal muscles are trained, it is well known that different exercises could elicit a greater activation of different abdominal muscles (Andersson et al. 1997); sit up is one of the most used exercises for rectus. Rectus abdominis (RA) is a muscle composed by more bellies interleaved with tendinous intersections; due to this anatomical feature different RA parts could be activated by the range of motion (Parfrey et al. 2008). The aim of this study was to analyze the emg activity of the rectus during sit up exercises performed with three different range of motion.

Methods: Four subjects were tested. Emg activity was recorded from six rectus abdominis bellies (upper URA, medium MRA and lower 
LRA part) and both rectus femoris (RF) with a Trigno wireless system (Delsys, $1000 \mathrm{~Hz}$ ) synchronized with an optoelectronic system that captured the positions of 6 markers bilaterally placed on the knee, hip joint center and acromion for shoulder segment (Vicon, $100 \mathrm{~Hz}$ ). Three different exercises, crunch $20^{\circ}(20)$, sit up $45^{\circ}(45)$ and full $\left(90^{\circ}\right)$ sit up (full) were performed. For each exercise five repetitions were recorded with $3 \mathrm{~s}$ rest in-between. The RMS of each belly/muscle was calculated in a $3^{\circ}$ range and normalized to the MVC value. Left and right sides and repetitions were averaged and compared among exercises.

Results: RA activation decreases by increasing exercise angle in full and 45 , whereas RF shows a parabolic profile with a peak in the middle of the exercise range of motion. When exercises were compared, some differences were detected: MRA shows a 50\% more activation in 45 and full compared with 20; URA activity is $40 \%$ higher in full than 45 in the $70^{\circ}-43^{\circ}$ range; $\mathrm{RF}$ activity is $55 \%$ lower in 20 compared with full and 45 and $27 \%$ higher in full than 45 in the $70^{\circ}-43^{\circ}$ range.

Conclusion: RA activity over the whole sit up range of motion decreases, whereas RF increases highlighting that small ranges should be used for training only RA. Moreover, in crunch exercise RF activity is negligible.

\section{References}

Andersson et al (1997) Abdominal and hip flexor muscle activation during various training exercises. Eur J Appl Phisiol Occup Physiol 75:115-123

Parfrey et al (2008) The effects of different sitand curl-up positions on activation of abdominal and hip flexor musculature. Appl Physiol Nutr Metab 33:888-895

\section{TP P}

\section{Balance training for rhythmic gymnastics}

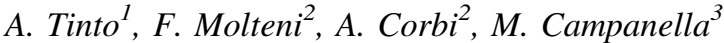 \\ ${ }^{1}$ University School of Motor and Sport Sciences, University of Turin, \\ Italy; \\ ${ }^{2}$ University School of Motor and Sport Sciences, Turin, Italy; \\ ${ }^{3}$ Instructor of Motor and Sport Sciences in Secondary School, MIUR
}

Purpose: Static balance is a coordination skill that has as its objective the maintenance of the center of gravity within the support base.

Rhythmic gymnastics, which is classified as a technical-motor type of discipline, requires that the athlete have excellent control of the condition of static and dynamic balance.

The aim of the research is to investigate the efficiency of a training program based on proprioception techniques.

Methods: The sample group was made up of 20 gymnasts, ages 11-13, with $6 \mathrm{~h}$ of training a week, distributed over 3 days.

The experimentation followed the girls for a period of 4 months. The sample group was divided into two subgroups, comprised of the same age and technical ability. The control group maintained the traditional structure of training while the work group utilized a method with a specific balance board (the use of instable and proprioception material for studying the difficulties of balance).

At the beginning and after a 4-month period of experimentation, the control group underwent the stork test, a method for evaluating the ability of gymnasts to maintain their balance in a monopodal support (left and right).

Results: The analysis of the obtained results showed more improvement in the work group with respect to the control group. All 20 subjects improved their values in the stork test but the gymnasts of the work group registered more significant increments (with average variations between the first and the second test of $225.7 \%$ in right support and $76.6 \%$ in left support).
Conclusion: The use of unstable surfaces during practice allows for exercising groups of specific muscles, able to guarantee optimal stabilization of the ankle in monopodal balance (Silvia et al. 2018).

Identifying a training course based on this methodology results, not only in an excellent method of practice with performance in mind, but it is also a precious instrument of (injury) prevention and a way of strengthening the muscular-ligament structure while undergoing a workload.

\section{Reference}

Silva PB, Oliveira AS, Mrachacz-Kersting N, Kersting UG (2018) Effect of wobble board training on movement strategies to maintain equilibrium on unstable surfaces. J Hum Mov Sci

\section{TP P}

\section{Teaching approaches and methodology of karate training: longitudinal study in athletes, ages 6-14}

\author{
M. Campanella ${ }^{1}$, A. Corbi ${ }^{2,}$ F. Molteni ${ }^{2}$, A. Tinto ${ }^{3}$ \\ ${ }^{1}$ Instructor of Motor and Sports Sciences in Secondary School, MIUR, \\ ${ }^{2}$ University School of Motor and Sport Sciences, University of Turin, \\ Italy; \\ ${ }^{3}$ University School of Motor and Sport Sciences, Turin, Italy
}

Purpose: Karate is categorized as a technical situation sport characterized by a high level of unpredictability (Manno).

The objective of the study is to identify a more efficient training methodology that is considered to be sensitive to the ability of 6-14 year olds for learning skills.

Methods: The sample group was made up of 54 children ages 6-14, ( 25 girls and 29 boys). The individuals in the study had two practices a week and the group was divided by age (T1: 6-11 and T2: 11-14). The focus of the training provided fine coordination and speed exercises, supplying an accurate study of basic techniques and action tactics. This was done through a series of exercise routines which is needed to transfer what is learned under competition conditions until the simulated fight.

The subjects were evaluated before and after the organization of the practice, through the administration of fitness tests: standing long jump, Sargeant test, shuttle runs, balance test.

Results: A comparison was made between the data from the fitness tests carried out by $\mathrm{T} 1$ and $\mathrm{T} 2$ at the end of the 6 -month course. It was based on the organization of a training program in view of the basic objectives and registered significant increases in both groups.

Knowing how to orient training means knowing how to identify methodologies of work better adapted to the age of the athletes and to favour a playful attitude, one of problem solving and free exploration for those from 6 to 8 years old while reaching situational unity, repetition series and competition exercises with children of 11-14 years of age.

Conclusion: To utilize a clear methodology that comes from a precise program is a needed requirement for organizing finalized technical skills at the performance. To know how to create a suitable training program allows for following the personal growth of the athlete without moving too quickly regarding development and preserving the integrity of the person.

References

Schmidt A, Lee TD (2012) Motor control and learning. Calzetti and Mariucci editor.

Weinek J (2009) The optimal training. Calzetti and Mariucci editor. 


\section{TP P}

\section{Pacing profiles of Olympic and IAAF World Championship long distance runners}

\author{
L. Filipas ${ }^{1}$, A. La Torre ${ }^{1,2}$, B. Hanley ${ }^{3}$ \\ ${ }^{1}$ Department of Biomedical Sciences for Health, Università degli \\ Studi di Milano, Milan, Italy; \\ ${ }^{2}$ IRCCS Istituto Ortopedico Galeazzi, Milan, Italy; \\ ${ }^{3}$ Carnegie School of Sport, Headingley Campus, Leeds Beckett \\ University, $U K$
}

Purpose: The aim of this study was to analyze the pacing profiles of Olympic and IAAF World Championship long distance finalists, including the relationship with their recent best times.

Methods: The times for each 1000-m split were obtained for 394 men and women in $5000 \mathrm{~m}$ and $10,000 \mathrm{~m}$ finals at five championships. Athletes' best times from the previous 32 months were also obtained. Similar pacing profiles were used by athletes grouped by finishing position in $5000 \mathrm{~m}$ races.

Results: Women adopted a more even pacing behavior, highlighting a possible sex-based difference over this distance. Pacing behavior over $10,000 \mathrm{~m}$ was more similar between men and women compared with over $5000 \mathrm{~m}$. The main difference between men and women was that in the men's $10,000 \mathrm{~m}$, as in the men's $5000 \mathrm{~m}$, more athletes were able to follow the leading group until the final stages. There were large or very large correlations between athletes' best times from the previous 32 months and their result; the fastest finishers also ran closer to their previous 32 months' best times.

Conclusions: Despite differences in pacing behavior between events, long distance runners should nonetheless stay close to the front from the beginning to win a medal.

Reference

Thiel C, Foster C, Banzer W, de Koning, J (2012) Pacing in Olympic track races: competitive tactics versus best performance strategy. J Sports Sci 30:1107-1115

\section{TP P}

\section{Kinesio taping does not improve the athletic performance of healthy master rowing}

\section{R. Barone ${ }^{1,2}$, E. Murazzi ${ }^{3}$, D. Zampaglione ${ }^{3}$, R. Fiore $^{3}$, G. Birritteri ${ }^{4}, F$. Cappello ${ }^{1,2,3}, F$. Macaluso ${ }^{4}$}

${ }^{I}$ Department of Experimental Biomedicine and Clinical Neurosciences (BioNeC), University of Palermo, Italy;

${ }^{2}$ Euro-Mediterranean Institute of Science and Technology (IEMEST), Italy;

${ }^{3}$ Medical Residency Program in Sport and Exercise Medicine, University of Palermo;

${ }^{4}$ SMART Engineering Solutions and Technologies (SMARTEST)

Research Center, eCampus University, Italy

Purpose: The purpose of this study was to investigate the effect of different applications of Kinesio tape on 1000-m ergometer-rowing performance in master athletes.

Methods: 10 healthy male rowing master athletes have been recruited for this study. Subjects were tested across 4 different sessions, on the distance of $1000 \mathrm{~m}$ at the ergometer-rowing. During the first trial, the subjects completed the distance without taping applied (WT trial). During the other three trial the Kinesio tape was applied, bilaterally, on the main muscles involved in the rowing: latissimus dorsi, biceps brachii and rectus femoris. Three different applications of Kinesio tapes were performed during the last three trials: placebo/wrong application (PLA trial); application that facilitates the technical gesture (FACI trial); application that inhibits the technical gesture (INHI trial). The order of applications was chosen randomly, although the same application was performed on all three muscles.

Results: In none of the three Kinesio tape applications (facilitate, inhibit and placebo/wrong) was observed a significant change of performance parameters measured: final time (WT 215.4 \pm 7.4; PLA $212.2 \pm 5.5$; FACI $213.1 \pm 5.0$; INHI $213.2 \pm 4.8 \mathrm{~s} ; \mathrm{p}>0,05)$, heart rate (WT $159.8 \pm 12.8 ;$ PLA $164.7 \pm 12.2$; $\quad$ FACI $166.0 \pm 10.3$; INHI $167.2 \pm 9.7$ beats $\left.\min ^{-1} ; \mathrm{p}>0,05\right)$ and power output (WT 282.1 \pm 30.5 ; PLA $294.3 \pm 22.4$; FACI $290.0 \pm 20.1$; INHI $290.6 \pm 19.5 \mathrm{~W} ; \mathrm{p}>0,05)$.

Conclusions: These results seems to indicate that the application of kinesio tape does not improve the performance of healthy rowing master athletes.

\section{TP P}

\section{Functional and mental training effects in archery sport performance}

\author{
R. Monzoni ${ }^{1}$, A. Capriotti $^{1}$, A. Federici ${ }^{1}$
}

Department of Biomolecular Sciences, Division of Exercise and Health Sciences, University of Urbino Carlo, Bo, Urbino, Italy

Purpose: In archery, use of elastic bands, along with bodyweight exercises (i.e. "functional training"), is widely accepted as a training strategy that effectively improves shooting technique (Lee and De Bondt 2005) because it allows to simulate the shooting action without bow and arrow. Indeed, Monzoni et al. (2017) demonstrated that athletes' performance in official competition increased significantly more after a functional training program aimed at improving strength (using elastic bands and calisthenics) and balance (exercises performed using the BOSU(C) instable support), compared to gym workout alone. Therefore, the aim of this investigation was to compare, in high level archers, the effects of two different training programs on the performance during official competitions, in which a group will performed a combination of mental and functional training. Methods: 20 male athletes with a minimum of 6 years of archery experience were recruited. Participants were assigned to one experimental group (EG) and to one control group (CG) with a balanced random order. The performance (i.e. total score of the participants) of each group was recorded during official competitions that took place just before and after the 6-month training intervention. All archers underwent a specific technical training program ( 3 sessions per week, 90 min per session) and functional training program ( 3 sessions per week, $90 \mathrm{~min}$ per session). In addition, the EG has performed a specific mental training program (2 session per week, 60 min per session) executing concentration exercises and analysis of personal technique.

Results: No statistical differences were found among the pre-training scores of the two groups, whereas the Ds resulted significantly different $(p<0.05)$. The $D$ of EG $(3.87 \pm 1.45 \%)$ increased significantly $(\mathrm{p}<0.001)$ more than the D of CG $(1.28 \pm 1.19 \%)$.

Conclusion: The results highlight that the use of functional training (using elastic bands, calisthenics and the BOSU ball, with the aim of simulating the shooting technique) combined with mental training (executing concentration exercises and analysis of personal technique) within an additional specific archery training program is effective in improving athletes' performance during official competitions. 


\section{References}

Dal Monte A (1983) La valutazione funzionale dell'atleta. Sansoni Editore, Firenze

Tursi D, Napolitano S (2014) Technical movements in archery. J Hum Sport Exerc 9(Proc1):S570-S575

Monzoni R, Lucertini F, Ferri Marini C, Federici A (2017) Functional training is effective in improving archery sport performance. J Hum Sport Exerc 12(PROC3):S1118-S1119

Keywords: Performance, Archery, Functional training, Mental training

\section{TP P}

\section{Physical performance in young footballers: effect of chronotype}

L. Galasso ${ }^{1}$, A. Mulè ${ }^{1}$, L. Castelli ${ }^{1}$, M. Cerri ${ }^{1}$, F. Esposito ${ }^{1,2}$, G. Coratella ${ }^{1}$, E. Limonta ${ }^{1,2}$, A. Caumo $^{1}$, A. Montaruli ${ }^{1,2}$, E. Roveda ${ }^{1,2}$

${ }^{1}$ Department of Biomedical Sciences for Health, University of Milan, Milan, Italy;

${ }^{2}$ IRCCS, Istituto Ortopedico Galeazzi, Milan, Italy

Purpose: The circadian rhythms influence our daily behavior, and people typically display preferences for activity at certain time of day. The circadian differences among individuals may be classified with the concept of chronotype. There are three chronotypes: Morning (MTypes), Evening (E-Types) and Neither Types (N-Types). M-Types have their best performances in the first part of the day. E-Types achieve their peak performance in the evening. N-Types have intermediate characteristics between the previous groups. The propensity toward diurnal or evening preferences can vary in different life periods and typically a shift towards eveningness occurs during adolescence. A relationship exists between circadian rhythms and physical performance, as many variables related to sports performance are linked to the chronotype.

Aim of the study was to verify whether there are performance differences relating to chronotypes in adolescent soccer players.

Methods: We recruited 80 male soccer players that filled in a Morningness-Eveningness Questionnaire (MEQ) for the assessment of chronotype. All participants underwent height and body mass measurements to calculate BMI $\left(\mathrm{kg} / \mathrm{m}^{2}\right)$. Thirty-nine participants, divided in M-Types $(n=13)$, E-Types $(n=13)$ and N-Types $(\mathrm{n}=13)$, performed three tests (Sargent Jump Test, Illinois Agility Test and 6 Minute Run Test) at two different times of the day, at 9.00 am and at $6.00 \mathrm{pm}$.

Results: The BMI values did not show statistically significant differences between groups. The data, analyzed by Mixed ANOVA, show statistically significant differences between chronotypes and time of the day (Sargent Jump, $\mathrm{p}<.05$; Illinois Agility $\mathrm{p}<.01 ; 6$ Minutes Run $\mathrm{p}<.01)$. In particular, for the three tests, E-Types showed a higher performance during the evening session than the morning session. Otherwise, M-Types performed better in the morning than in the evening session.

Conclusions: These results show that the chronotype is able to influence the exercise performance in adolescent soccer players.

\section{References}

Reilly T et al (2007) Diurnal variation in temperature, mental and physical performance, and tasks specifically related to football (soccer). Chronobiol Int 24(3):507-519

Montaruli A et al (2017) The circadian typology: role of physical activity and melatonin. Sport Sci Health

\section{TP P}

Predictors of six minute walking test among children of Italian primary school: a cross sectional study

\author{
M.V. Puci ${ }^{1}$, M. Vandoni ${ }^{2}$, L. Correale ${ }^{2}$, C. Galvani ${ }^{3}$,

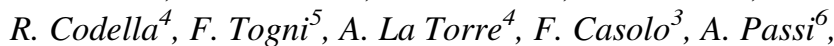 \\ C. Orizio ${ }^{5}$, C. Montomoli ${ }^{1}$
}

${ }^{1}$ Unità di Biostatistica ed Epidemiologia Clinica, Dipartimento di Sanità Pubblica, Medicina Sperimentale e Forense, Università di Pavia, Pavia, Italia;

${ }^{2}$ Laboratorio di Attività Motoria Adattata (LAMA), Dipartimento di Sanità Pubblica, Medicina Sperimentale e Forense, Università di Pavia, Pavia, Italia;

${ }^{3}$ Laboratorio di Fisiologia Sperimentale, Dipartimento di Psicologia, Università Cattolica del Sacro Cuore, Milano, Italia;

${ }^{4}$ School of Exercise Sciences, Department of Biomedical Sciences for Health, Università degli Studi di Milano, Milan, Italy;

${ }^{5}$ Corso di Laurea in Scienze Motorie, Dipartimento di Scienze Cliniche e Sperimentali, Università degli studi di Brescia, Brescia, Italia;

${ }^{6}$ Corso di Laurea in Scienze Motorie, Università degli Studi dell'Insubria, Varese, Italia

Purpose: The 6-minute walking test (6MWT) is a simple assessment tool to evaluate exercise capacity. Existing six-minute walking distance (6MWD) prediction equations were developed using healthy children [1]. Our aim was to evaluate the predictors of 6MWD in healthy Italian children.

Methods: In our cross-sectional study, we performed 6MWT in 5614 children aged 6-11 years between November 2016 and May 2017. We used Student $t$ test for independent data or Mann-Whitney test to compare the quantitative variables between age groups and gender. We assessed a multiple linear regression model to estimate a prediction equation for the 6MWD. Performance and goodness of fit of the models were assessed using the root-mean-square error and R-squared measures. A p-value $<0.05$ was considered significant.

Results: Mean distance walked for all participants was $595.5 \pm 80.9 \mathrm{~m}$ (range $360-800 \mathrm{~m}$ ). We observed significant differences about gender and age: males walked longer distances than females $(598.8 \pm 83.9 \mathrm{~m}$ vs $592.1 \pm 77.6 \mathrm{~m} ; \mathrm{p}=0.0016)$, while in younger children (6-8 years) correlations between 6MWD and demographic/anthropometric data were significantly higher compared with older children $(9-11$ years; $p<0.0001)$. The regression model showed that age, gender and height were positively related to 6MWD, while weight was negatively related $(6 \mathrm{MWD}=107.91+25.02 \times$ age $($ years $)+7.52 \times$ gender $(M)+2.62 \times$ height $(\mathrm{m})-2.25 \times$ weight $(\mathrm{kg})$.

Conclusions: Our study confirmed that demographic and anthropometric characteristics can influence the test performance in healthy subjects. In our model, age, gender, height and weight significantly add information and should be taken into account in this particular growth phase. The 6MWT is feasible in education's environment: school could be a main setting to implement primary prevention by using motor assessment.

\section{Reference}

1. Ben SH et al (2009) Reference equation for 6-min walk distance in healthy North African children 6-16 years old. Pediatr Pulmonol 44(4):316-324 


\section{TP P}

\section{Reliability of ultrasound assessment of diaphragm structure and function}

\author{
M. Borrelli, S. Rampichini, C. Doria, E. Limonta, S. Longo,
} S. Shokohyar, G. Coratella, E. Roveda, E. Cè, F. Esposito

Department of Biomedical Sciences for Health, Università degli Studi di Milano, Italia

Purpose: In the light of the raising interest in respiratory muscle training, additional quantitative, reliable and sensitive indicators able to describe training-induced changes in diaphragm muscle structure and function are needed together with traditional spirometric parameters $[1,2]$. This study aimed at investigating the intra-, interday and inter-operator reliability and sensitivity of diaphragm thickness and excursion measurements in healthy young adults.

Methods: Eleven healthy subjects $(6$ males and 5 females; age $26.6 \pm 4.6$ years, body mass $67.1 \pm 15.0 \mathrm{~kg}$; stature $1.74 \pm 0.11 \mathrm{~m}$ ) were enrolled. Diaphragm thickness was measured by ultrasound in B-mode during spontaneously breathing at the end of inspiration (TEI) and expiration (TEE). Excursion amplitude (EA) was measured in M-mode during breathing at $1 \mathrm{~Hz}$. Measurements were taken twice (intra-day reliability) by three expert operators (inter-operator reliability) during two different sessions (inter-day reliability). For each parameter, the reliability was expressed as Chronbach's $\alpha$ and the percentage standard error of measurement (SEM\%). The sensitivity was calculated as the percentage of the minimum detectable change (MDC\%).

Results: TEI and TEE were $1.71 \pm 0.35$ and $1.37 \pm 0.31 \mathrm{~cm}$, respectively. $\alpha$ and SEM $\%$ in TEI and TEE ranged from 0.862 to 0.987 and from 1.8 to $7.5 \%$. MDC\% spanned from 3.6 to $14.7 \%$. EA ranged from 3.15 to $3.68 \mathrm{~cm}$. $\alpha$ and SEM\% in EA were between 0.765 and 0.954 and between 5.0 and $13.1 \%$, respectively, while MDC\% ranged from 9.7 to $25.6 \%$.

Conclusions: These findings suggest that TEI, TEE thickness and EA could be considered as reliable quantitative indicators of possible training-induced modifications in the diaphragm muscle.

\section{References}

Sarwal A, Walker FO, Cartwright MS (2013) Neuromuscular ultrasound for evaluation of the diaphragm. Muscle Nerve 47(3):319-329

Harper CJ, Shahgholi L, Cieslak K, Hellyer NJ, Strommen JA, Boon AJ (2013) Variability in Diaphragm motion during normal breathing, assessed with B-mode ultrasound. J Orthop Sports Phys Ther 43(12):927-931. https://doi.org/10.2519/jospt.2013.4931.

\section{TP P}

\section{Do life skills influenced the technical capacity of young soccer player?}

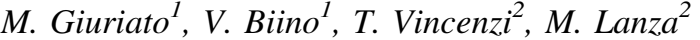 \\ ${ }^{1}$ Department Human Science, Universita' degli Studi di Verona; ${ }^{2}$ Department of Neurosciences, Biomedicine and Movement Sciences, University of Verona, Verona, Italy}

Introduction: Life Skills (LS) are personal and relationship ability that allow to face efficiently problems, and stress depression of daily life. LS (relationship, autonomy, competence) (Hodge et al. 2012) results fundamental during childhood to permit an adequate growth like soccer player and like person. The aim of the study it was investigated if the LS influenced the technical capacity of young soccer player

Materials and methods: Twentyone young male soccer player of professional team (age $=10.86 \pm 0.36$ years; $\mathrm{H}=1.47 \pm 0.06 \mathrm{~m}$; $\mathrm{W}=38.0 \pm 4.9 \mathrm{~kg} ; \mathrm{BMI}=17.62 \pm 1.44)$. Technical capacity was assessed with Loughborough Soccer Passing Test (LSPT), furthermore stratified in three groups per test results (Low, Normal, Fast). LS was assessed with The Group Environment Questionnaire (GEQ), it was composed of four item (ATG-T; ATG-S; GI-T; GI-S). Every test it was performed in three time (February; April, June), and stratified in three Time Group (T1 = February; T2 = April; T3 = June)

Results: One way ANOVA between LSPT and Time Group suggest that the relationship between $\mathrm{T} 2-\mathrm{T} 3$ and $\mathrm{T} 1-\mathrm{T} 2$ it was significant, $(\mathrm{T} 1: \quad 62.17 \pm 9.42 \mathrm{~s}, \quad \mathrm{~T} 2: \quad 67.20 \pm 12.39 \mathrm{~s}, \quad \mathrm{~T} 3: \quad 61.03 \pm 11.59$, $\mathrm{p}<0.05$ ) but not between T1-T3; furthermore One way ANOVA between GEQ and Time Group, suggest a significance between T2$\mathrm{T} 3$ in GI-S item (T1: $31.19 \pm 3.53, \mathrm{~T} 2: 31.10 \pm 3.45$, T3: $31.86 \pm 3.07)$; furthermore, Two way ANOVA between LSPT and GEQ suggested that there isn't any significance. Finally, it was analysed difference between PREvsPOST (T1-T2 vs T2-T3) with T Test in every single group of LSPT, and the results suggest significant only in the Fast group $(\mathrm{PRE}=58.54 \pm 5.06$, POST $=55.13 \pm$ $6.06 \mathrm{~s}, \mathrm{p}<0.05)$.

Discussion: An improvement in LSPT between T2-T3 and T1-T2 it was recorder, furthermore the analyses of GEQ shown how the children after experimental protocol (T2-T3) improved in cohesion of group (GI-S). Finally, only player with high technical ability (Fast group) improved significantly from PRE (T1-T2) to POST (T2-T3) Reference

Hodge K, Danish SJ (2012) Developing a conceptual framework for life skills interventions. Counsel Phychol

\section{TP P}

\section{Evaluation of performance in young ballet dancers: comparison of two different training methods}

\author{
R. Polito ${ }^{1}$, A. Elce El, G. Di Rauso $^{2}$, F. Guarino ${ }^{2}$, \\ A. Sangiorgio ${ }^{2,3}$, A. Del Pizzo ${ }^{5}$, E. Nigro ${ }^{3}$, A. Daniele ${ }^{1}$
}

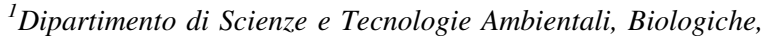
Farmaceutiche, Università della Campania "Luigi Vanvitelli", Caserta, Italy;

${ }^{2}$ Università Telematica Pegaso, Facoltà di Scienze Umanistiche, Napoli;

${ }^{3}$ Dipartimento di Medicina e di Scienze della Salute "Vincenzo Tiberio" Università degli Studi del Molise, Campobasso;

${ }^{4}$ Ceinge, Biotecnologie Avanzate Scarl, Napoli;

${ }^{5}$ University of Pisa, Physics Department, Pisa

Purpose: Ballet dance is one of most popular discipline chosen by girls during childhood and adolescence. In these years a new method of training featured of a mix of calisthenics and classical exercises was introduced in Italy. Given its peculiar characteristics, Dance sport represents a feasible, attractive and alternative physical activity to preserve cognitive and physical functions during aging. Increased self-esteem, social contact and psychophysical wellness significantly ameliorate the quality of life during aging. Objective of our study was to analyze the performances of a total of 60 young dancers aged from 8 to 14 years old divided in two groups: thirty of them trained for 1 year adopting classic dance training, instead the other thirty trained 
for a year with a mixed training, characterized by exercises of rhythmic gymnastics alternating exercises from classical dance.

Methods: The two groups were evaluated at time 0 and after 1 year with ten performance tests and joint flexibility and mobility tests validated by Comitato Olimpico Nazionale Italiano (CONI). Anthropometric data and life style habits were collected. $t$ test analysis was performed on collected data.

Results: The subjects included in the study trained with a mean frequency of $10 \mathrm{~h}$ a week, on average for at least a year since the beginning of the study. Anthropometric parameters resulted comparable for height, weight and BMI to the WHO growth charts in relation to age. After 1 year of training, the mixed workout group had an increased dominant wrist circumference compared to the classic workout group $(13.24 \pm 1.24 \mathrm{~cm}$ in 2016 versus $13,93 \pm 1.26 \mathrm{~cm}$ in 2017; $\mathrm{p}=0.011)$. We observed that mixed workout group showed more significant improvements in 8 of the 10 tests administered (30 min running, 60 min running, push up, chin up, sit up, sergeant, ABD $90^{\circ}$, rope 30 ).

Conclusions: We observed a regular growth in both groups. A mixed workout training seem to determine a better performance in the dancers after a year of training compared to classic training. In mixed workout group the training may influence the increase of bone mass in the athletes, revealed by measurement of dominant wrist.

\section{TP2 P}

\section{The relative age effect in elite Italian team sports emerged only in the first years of career}

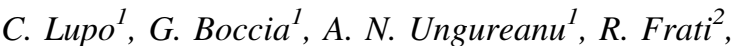 R. Marocco $^{2}$, P. R. Brustiol}

${ }^{1}$ NeuroMuscularFunction | Research Group, School of Exercise and Sport Sciences, SUISM; Department of Medical Sciences, University of Torino, Turin, Italy;

${ }^{2}$ School of Exercise \& Sport Sciences, SUISM, University of Torino, Turin, Italy

Purpose: Athletes are grouped according to their year of birth to arrange young athletes in sports competitions. Therefore, older athletes (first weeks of a year) could be advantaged compared with relatively younger athletes (i.e., last weeks of the same year) in sport performance (Cobley et al. 2009), and in the process of sport talent identification (Helsen et al. 2005). The present study aims to investigate the presence of the Relative Age Effect (RAE; i.e., an asymmetry in birth-date distribution) in elite Italian team sport championships, hypothesizing that this phenomenon is evident for overall samples, as well as younger and older player subgroups.

Methods: For this purpose, 642, 572, 337, and 450 professional players were considered from the two highest male Italian basketball (i.e., Serie A1, Serie A2), rugby (i.e., Eccellenza, Serie A), volleyball (i.e., Serie A1, Serie A2), and water polo (i.e., Serie A1, Serie A2) Championships, respectively. Players were classified in relation to the week of birth (i.e., occurrence of weeks existing between the first week of year and that of the player's birth) and analyzed using Poisson regressions (index of discrimination, ID; $p \leq 0.05$ ). The analyses were applied for single team sport, considering their entire player samples as well as younger (i.e., $\leq 25^{\circ}$ percentile of the ages; basketball: $\leq 19$ years; rugby: $\leq 21$ years; volleyball: $\leq 22$ years; water polo: $\leq 19$ years) and older (i.e., all other players) sub-groups separately.

Results: The RAE emerged for each team sport (ID): basketball: 1.57 $(\mathrm{p}<0.001)$; rugby: $1.34(\mathrm{p}=0.04)$; volleyball: $1.48 \quad(\mathrm{p}=0.04)$; water polo: $1.45(\mathrm{p}=0.02)$. However, considering younger and older subgroups, RAE is significant only for the first one (basketball: 2.38 , $\mathrm{p}<0.001$; rugby: $2.07, \mathrm{p}=0.01$; volleyball: $2.05, \mathrm{p}=0.03$; water polo: $1.84, \mathrm{p}=0.04)$, whereas no significant RAE emerged for older counterparts.

Conclusions: Team sport players born close to the data of selection might have more chances to be selected in the highest competition levels. However, this trend is confirmed only for the young subgroups, speculating that players born in the rest of the year have the opportunity to play at the highest level successively. Finally, limitations into the talent identification process can emerge, and coaches and scouts should consider RAE, in order to select the promising players in the long-term rather than the best performers at the time of selection.

\section{References}

Cobley et al (2009) J Educ Psychol

Helsen et al (2005) J Sports Sci

\section{TP P}

\section{Peak torque in concentric contractions is differently determined in knee flexors and extensors}

\author{
P.R. Brustio ${ }^{1}$, G. Boccial ${ }^{1}$, G. Buttacchio ${ }^{2}$, M. Calabrese $^{2}$, \\ R. Casale $^{2}$, A. Rainoldi ${ }^{1}$
}

${ }^{1}$ NeuroMuscularFunction Research Group, School of Exercise and Sport Sciences, Department of Medical Sciences, University of Turin, Turin, Italy;

${ }^{2}$ Habilita Care and Research Rehabilitation Unit, Zingonia, Bergamo, Italy

Purpose: Measuring the maximal isometric voluntary contraction (MVC) represents an easy method for the muscle function assessment. However, the rate of force development (RFD) is a relative new neuromuscular parameter that plays an important role in sport performance as it represents the ability to carry out rapid and forceful movements. The aim of this study was to evaluate the impact of MVC and RFD on concentric isokinetic contractions in a sample of elite young soccer players.

Methods: Twenty-two elite young soccer players (mean age $M=17 \pm 1$ years) were enrolled in the study. Subjects were seated on an ergometer and performed (i) two 3-s MVC; (ii) rapid isometric contractions with a variety of force levels $(20-40-60-80-100 \%$ of MVC); and (iii) three concentric isokinetic contractions at $240 \% \mathrm{~s}$. Measures were obtained for both dominant and non-dominant limbs, and for both knee extensors and flexors.

Results: MVC and RFD altogether accounted for $40.8 \%$ of the variance in concentric isokinetic torque $\left(\mathrm{F}_{2,39}=12.773 ; p<0.001\right)$ in knee extensors and for $48.3 \%$ of the variance in concentric isokinetic torque $\left(\mathrm{F}_{2,38}=16.790 ; p<0.001\right)$ in knee flexors. Only RFD significantly accounted for the variance in concentric isokinetic torque exerted by knee extensors $(\beta=0.598 ; p=0.005)$, while only MVC significantly accounted for the variance in concentric isokinetic torque exerted by knee flexors $(\beta=0.487 ; p=0.003)$.

Conclusions: These results showed that the peak torque exerted in concentric isokinetic contractions is related to different mechanisms in knee extensors with respect to flexors. Indeed, in knee extensors the peak torque in concentric contractions was mainly explained by RFD, while in knee flexors by MVC. These findings suggest that, to increase concentric torque expression, a different strategy should be adopted for knee extensor and flexor muscles. While explosive training could be more effective in knee extensors, maximal strength training (e.g., Nordic Hamstring) could be more effective in knee flexors. 


\section{TP P}

Anthropometric profile and body composition in competitive swimmers: effects of measurement technique

\author{
L. Beratto ${ }^{1,2}$, S. Cattaneo $^{3}$, L. Corapi ${ }^{4}$, F. Abate Daga ${ }^{2,5}$, \\ M. Ponzano ${ }^{6}$, M. Gollin ${ }^{2,7}$
}

${ }^{1}$ Experimental Medicine and Therapy, Department of Clinical and Biological Sciences, University of Turin, Italy;

${ }^{2}$ Adapted Training and Performance Laboratory, University School of Motor and Sport Sciences, University of Turin, Italy;

${ }^{3}$ University of Turin, Italy;

${ }^{4}$ University of Turin, Italy;

${ }^{5}$ Department of Medical Sciences, University of Turin, Italy;

${ }^{6}$ Department of Kinesiology, University of Waterloo, Waterloo, Canada;

${ }^{7}$ Department of Clinical and Biological Sciences, University of Turin, Italy

Purpose: Swimming performance is strongly influenced by technique. Anthropometric characteristics and body composition are important factors to reduce drag and improve physical performance in swimmers. Generally, low fat mass values lead to better performances. However, a greater fat mass may improve floatation and reduce energy expenditure. The aims of this study were to describe the anthropometric characteristic of competitive swimmers and to evaluate differences among males and females.

Methods: Competitive swimmers were recruited from an Italian semi-professional teams. Body mass index (BMI) was calculated for all swimmers. Seven skinfold measurements were perm using a Holtain skinfold caliper. Each athlete underwent a bioelectrical impedance analysis (BIA) at a single frequency $(50 \mathrm{~Hz})$. Two-way analysis of variance (ANOVA) and Fisher's LSD post hoc were used to assess differences between sex and specialities regarding BMI, skinfolds and BIA parameters. The level of significant was set at $\mathrm{p}<.05$. All statistical analyses were performed with SPSS software version 25 .

Results: Data were collected from 36 competitive athletes (16 males and 20 females, age $19 \pm 4$ years; height $176 \pm 10 \mathrm{~cm}$; weight $66 \pm 10 \mathrm{~kg}$ ) of which 11 (4 males, 7 females) were distance swimmer, 13 (6 males, 7 females) middle distance and 12 (6 males, 6 females) sprinters. BMI did not present significant differences between specialities $(p=.089)$ and $\operatorname{sex}(p=.128)$. Males have a higher fat-free mass percentage than females when assessed with both skinfold $(\mathrm{p}<.0001)$ and BIA $(\mathrm{p}=.0004)$. Skinfold calipers measurement did not reveal any differences between specialities $(p=.560)$. Differences between specialities were obtained when performing BIA $(\mathrm{p}<.0001)$. Females have a higher fat mass percentage than males when assessed with both skinfold $(p<.0001)$ and BIA $(\mathrm{p}<.0001)$. No differences between specialities are present when assessed with both skinfolds $(\mathrm{p}=.879)$ and BIA $(\mathrm{p}=.765)$.

Conclusion: BMI did not differ between sex and specialities. Males swimmers have a higher fat-free mass and a lower fat mass than females regardless of speciality and measurement technique. BIA showed a difference between specialities regarding fat-free mass. This study highlighted discrepancies between the different techniques to assess body composition. Future studies are required to define the best measurement technique among competitive swimmers.

References

Martínez S, Pasquarelli BN, Romaguera D, Arasa C, Tauler P, Aguiló A (2011) Anthropometric characteristics and nutritional profile of young amateur swimmers. J Strength Cond Res 25(4):1126-1133
Zuniga J, Housh TJ, Mielke M, Hendrix CR, Camic CL, Johnson GO, Housh DJ, Schmidt RJ (2011) Gender comparisons of anthropometric characteristics of young sprint swimmers. J Strength Cond Res 25(1):103-108

\section{ADAPTED PHYSICAL ACTIVITY}

\section{AP P \\ Baskin-a basketball-based sport for disabled athletes: a role weight's analysis}

\section{Sisti, S. Amatori, R. Bensi, A.R. Calavalle, M. Gervasi, R. Lauciello, M.B.L. Rocchi}

Department of Biomolecular Sciences, University of Urbino "Carlo Bo", Italy

Purpose: A new example of sport that reflects the principles expressed by reverse integration is Baskin, a basketball-based sport for disabled athletes. Baskin assigns roles according to disability level, ranging from 1 to 5 . Athletes with a more severe handicap have more regulation facilities, whilst able-bodied athletes have a series of limitations and rules that oblige them to interact with disabled athletes. In 2016, Baskin involved over 4000 people. The aim of this study was to verify if the game rules assign the right weights to the actions of each role.

Methods: 90 game reports, involving 395 players, were taken into account for this study. A mixed model with interaction was used to verify if the scores made according to the role independent from the outcome, were different from the roles themselves.

Results: The roles 1 and 2, followed by the role 5, have the higher weight on the result of the match. Roles' scores are significantly different from each other $(\mathrm{p}<0.001)$. In particular, role take account of major differences between player scores $\left(p<0.001 ; \eta^{2}=0.272\right.$, large effect); secondly, the match outcome is significantly associated with the score of the players $\left(p=0.033 ; \eta^{2}=0.002\right.$, very small effect). The interaction shows how the outcome (victory or defeat) changes the ratio between the scores of the individual roles $\left(\mathrm{p}<0.001 ; \eta^{2}=0.015\right.$, small effect). Post-hoc test relieved that roles $1,3,4$ and 5 showed non-significant differences between mean scores (won or lost), whilst role 2 scores were associated to the outcome of the match $(\mathrm{p}<0.001)$.

Conclusions: It should be useful to better define how to identify and classify the roles according to skills since it seems that above all roles 1 and 2 have the greatest weight on the final score. In addition, some changes to the regulation in order to make more homogeneous the scores deriving from the various roles could be proposed, although the authors agree that the disabled athletes should have primary importance in the game. Data examined showed how much the contribution of the disabled is important in the outcome of the matches and how often this can be decisive. Baskin makes it possible to formulate a different idea of sport.

\section{Reference}

Hutzler Y, Chacham-Guber A, Reiter S (2013) Psychosocial effects of reverse-integrated basketball activity compared to separate and no physical activity in young people with physical disability. Res Dev Disabil 34:579-587 


\section{AP P}

Exercise, inflammation and vascular function in aging and obesity

\section{A. Pedrinolla ${ }^{1,2}$, E. Kirmizi ${ }^{3}$, F. Moschetta ${ }^{2}$, M. Zardini ${ }^{2}$, D. Rudi ${ }^{l}$, E. Bacchi ${ }^{2}$, P. Moghetti ${ }^{2}$, M. Lanza ${ }^{1}$}

${ }^{1}$ Department of Neuroscience, Biomedicine and Movement Science, University of Verona, Verona, Italy;

${ }^{2}$ Division of Endocrinology, Diabetes and Metabolism, Department of Medicine, University of Verona and Hospital Trust of Verona, Verona, Italy;

${ }^{3}$ Department of Physiology, Faculty of Medicine, Uludag University, Eskisehir City Hospital, Eskisehir, Turkey

Purpose: Aging is a non-modifiable process. Obesity is a reversible condition. However, both are characterized by a low-grade inflammatory profile and vascular dysfunction. Exercise is a nonpharmacological strategy able to counteract the negative effect of aging as well as of obesity. Nonetheless, its long-term effect on inflammation and vascular function in obese and non-obese elderly individuals is still mate of debate. The aim of the study was to understand the effect of regular exercise on inflammatory profile and vascular function in non-obese and obese elderly individuals.

Methods: Seventy individuals who attended a structured exercise program $(30 / 40-\mathrm{f} / \mathrm{m} ; 75 \pm 5$ years; $5 \pm 2$ years of regular training) were enrolled in the study and tested for vascular function (flowmediated dilation; FMD) and inflammatory profile (plasma CRP, IL$1 \beta$, IL-1ra, IL-6, IL-8, IL-10, TNF- $\alpha$, MCP-1). Subjects were stratified for age and BMI. Correlations between age, BMI and the measured variables were investigated.

Results: Inverse correlations were found between age and IL $\beta$ (r $-0.232 ; \mathrm{p}<.05)$; IL-1ra $(\mathrm{r}-0.181 ; \mathrm{p}<.05)$; IL-6 ( $\mathrm{r}-0.255$; $\mathrm{p}<.05)$; and IL-8 $(\mathrm{r}-0.248 ; \mathrm{p}<.05)$. Direct correlations were found between BMI and CRP (r 0.155; p <.05), MCP-1 (r 0.217; $\mathrm{p}<.05)$; and TNF- $\alpha$ ( $\mathrm{r} 0.184 ; \mathrm{p}<.05)$. An inverse correlation was also found between BMI and FMD ( $\mathrm{r}-0.433$; $\mathrm{p}<.01)$

Conclusion: Sustained, regular exercise can counteract the deleterious effects of aging but, although obese subjects showed a good vascular function, exercise cannot counteract obesity-induced effects on vascular function and inflammatory profile. It appears that if exercise is not accompanied by an important weight lost, its effect on the inflammatory profile is poor. However, further research is needed to confirm these findings.

References

Taddei S, Galetta F, Virdis A, Ghiadoni L, Salvetti G, Franzoni F et al (2000) Physical activity prevents age-related impairment in nitric oxide availability in elderly athletes. Circulation 101:2896-2901

Ellis A, Crowe K, Lawrence J (2013) Obesity-related inflammation: implications for older adults. J Nutr Gerontol Geriatr 32(4):263-290
86 AP P

Adapted physical activity programs for a subject with relapsing-remitting multiple sclerosis: a case report study

\author{
L. Marin ${ }^{1,2}$, A. Gaudio ${ }^{1}$, I. Buil ${ }^{1}$, F. Gentile ${ }^{1}$, M. Febbi ${ }^{2}$, \\ S. Ottobrini ${ }^{1}$ \\ ${ }^{1}$ Laboratory of Adapted Motor Activity (LAMA), Department \\ of Public Health, Experimental Medicine and Forensic Science, \\ University of Pavia, Pavia, Italy; \\ ${ }^{2}$ Technology for Sports Medicine and Rehabilitation, Department \\ of Industrial Engineering, University of Rome "Tor Vergata", Rome, \\ Italy
}

Purpose: More than 2.5 million people worldwide suffer from Multiple Sclerosis (MS). Many studies showed that the adapted physical activity (APA) allows the maintenance and improvement of the patient's functional independence, bringing several benefits on the main symptoms such as reduction of postural disorders and sense of fatigue. It also improves the quality of life by decreasing the sense of discomfort and depression. This study aimed to evaluate the effects of a tailored 3 years APA program on the functional abilities and life satisfaction in a subject with Relapsing-Remitting (RR) MS.

Methods: From October 2014 to June 2017 a case report study on a 30-year-old man, affected by RR MS for 8 years, was conducted. Each annual program was aimed to increase/maintain balance, mobility, ambulation, strength and fatigue management. Each program was composed by 3 mesocycles, lasting 10 weeks each, and a 4-week break.

At the beginning and at the end of each year $\left(\mathrm{T}_{0}, \mathrm{~T}_{1}\right)$ a set of evaluations was carried out to evaluate: strength (Handgrip Test, Medical Ball Launch), walking abilities (6 min-Walk Test-6MWT, 30-meters Test), level of satisfaction about many aspects of the subject's life (SAT-P). During all the tests, the Borg scale was used to monitor the perceived exertion.

Results: The relapses occurred in the 3 years of the APA program required many adaptations and prevented the execution of all the tests over 3 years. The results of the tests showed an improvement of the strength, considering both each year of activity and the whole 3 years. Regarding the walking abilities, the 6MWT and the 30-meter Test, both performed only in the first 2 years, showed a slight worsening in the first year and an improvement in the second year. The two factors of physical and psychological functioning of the SAT-P showed an increase from the beginning to the end of the first year and (only for physical functioning) of the second year. A decrease was observed in the third year, probably because of the worsening of the pathology. Conclusions: For this subject with RR MS, the APA intervention provided encouraging results and it would be interesting to study this topic in a larger sample and in various MS forms.

\section{References}

Compston A, Coles A (2008) Multiple sclerosis. Lancet 372:1502-1517

Motl RW, McAuley E, Snook EM (2005) Physical activity and multiple sclerosis: a meta-analysis. Mult Scler 11:459-463 


\section{AP P}

Walking groups in subjects affected by schizophrenia: a winning strategy against sedentary life style?

\author{
S. Mandini ${ }^{1,2}$, M. Morelli ${ }^{1}$, R. Bivi ${ }^{3}$, L. Simani ${ }^{3}$, \\ Elisa Mori ${ }^{1}$, M. Cristofori ${ }^{2}$, G. Grazzi ${ }^{1,2}$, G. Mazzoni ${ }^{1,2}$ \\ ${ }^{1}$ Center of Biomedical Studies Applied to Sport, University \\ of Ferrara, Ferrara, Italy; \\ ${ }^{2}$ Public Health Department, AUSL Ferrara, Ferrara, Italy; \\ ${ }^{3}$ Public Mental Health Department, AUSL Ferrara, Ferrara, Italy
}

Purpose: Subjects with schizophrenia are more likely to be sedentary, overweight and at higher risk of diabetes, hypertension and dyslipidaemia than general population. The life expectance is reduced by $7-24$ years and about $60 \%$ of mortality is due to cardiovascular diseases. Aim of this study is to include a group of sedentary adults with schizophrenia in a program of regular physical activity (PA) and to evaluate the effects of PA on cardiovascular function.

Methods: 12 sedentary subjects with schizophrenia recruited by the local Public Mental Health Department were included in the study (age $45 \pm 9$ ). Height, weight, BMI, waist circumference and blood pressure were measured. To assess the baseline walking speed subjects performed the $1-\mathrm{km}$ test. Heart rate (HR) was monitored continuously during the test. These evaluations were performed at baseline and after 6 -months of walking. The subjects were guided by an exercise physiologist to walk $1 \mathrm{~h}$ twice/week.

Results: 11 subjects complete the program. The subject not included in the finishers was hospitalized during the measurement period; he did not leave the project. The adherence at the program was of $95 \%$. After 6-months of walking the distance covered in each training session goes from 4 to $5 \mathrm{~km}$ (increase of $25 \%$ ). No significant changes in weight and BMI were observed. A degree of $1.2 \mathrm{~cm}$ in waist circumference was observed. The systolic pressure goes from $126 \pm 14$ to $120 \pm 10(\mathrm{p}<0.05)$. Maximum HR decrease significantly of $8.4 \mathrm{bpm}(\mathrm{p}<0.05)$. In conclusions, the study population moved from sedentary to physically active. An excellent acceptance towards the walking group was observed and confirmed by the highest adherence to the training session. In addition to an improvement in cardiovascular function and to a reduction in risk factors, an increase in the capacity for memory and attention in daily activities were observed.

\section{References}

De Hert $M$ et al (2009) Metabolic syndrome in people with schizophrenia: a review. World Psychiatry 8(1):15-22

Brenda W (2018) Metabolic syndrome in psychiatric patients: overview, mechanisms, and implications. Dialogues Clin Neurosci 20(1):63-73

Lindamer LA et al (2008) Assessment of physical activity in middle aged and older adults with schizophrenia. Schizophr Res 104(1-3):294-301

Chiaranda $G$ et al (2012) Peak oxygen uptake prediction from a moderate, perceptually regulated, $1-\mathrm{km}$ treadmill walk in male cardiac patients. J Cardiopulm Rehabil Prev 32(5):262-269

\section{AP P}

Comparison between dual-energy $\mathrm{X}$-ray absorptiometry and skinfold thickness in assessing percentage of body fat in athletes with unilateral lower limb amputation

\author{
V. Cavedon ${ }^{1}$, A. Pedrinolla ${ }^{1}$, M. Venturelli ${ }^{1}$, C. Nichele ${ }^{2}$, \\ C. Zancanaro ${ }^{1}$, C. Milanese ${ }^{1}$ \\ ${ }^{I}$ Department of Neurological, Biomedical and Movement Sciences, \\ University of Verona, Verona, Italy; \\ ${ }^{2}$ Private practice in sport science and rehabilitation, Vicenza, Italy
}

Purpose: Anthropometric equations are an accessible and cost-effective method to assess percentage of body fat $(\% \mathrm{BF})$ in athletic populations, but it is still unclear how the lower limb amputation may affect their reliability in athletes with physical impairments. The aim of this study was to compare, in amputee soccer players, \%BF estimated with several commonly used equations and \%BF measured with dual-energy X-ray absorptiometry (DXA, Hologic).

Methods: The \%BF was assessed in 10 men amputee soccer players aged $33.9 \pm 11.9$ years with unilateral transfemoral $(n=5)$ or transtibial $(n=5)$ lower limb amputation. Anthropometric equations were used to predict body density in the same athletes with existing regression equations established for able-bodied subjects [i.e., Durnin and Womersley (1974), Jackson and Pollock 7-sites (1978), Sloan and Weir (1970), Wilmore and Behnke (1969) and Katch e McArdle (1973)] and converted to \%BF according to Siri (1961). Pairedsample $t$ test was used to compare anthropometric equation-derived $\% \mathrm{BF}$ and DXA measurements. The coefficient of determination was computed, and Bland-Altman analysis was used to test the agreement between methods.

Results: All the other considered regression equations significantly underestimated \%BF by $2.7-6.0 \%$, but the Durnin and Womersley equation which significantly overestimated $\% \mathrm{BF}$ by $4.0 \%$. The highest (0.805) coefficient of determination (adjusted) was found in the Wilmore and Behnke equation while the lowest value $(0.422)$ was found in the Durnin and Womersley equation. The standard error of estimate ranged from 2.37 (Wilmore and Behnke equation) to 4.08 (Durnin and Womersley equation). Further comparative studies are required to confirm or refine the accuracy of practical, non-invasive methods for monitoring \% BF in this population. Impairment specific equations may be needed in in amputee soccer players with unilateral lower limb amputation.

\section{Reference}

Willems A et al (2015) Dual-energy X-ray absorptiometry, skinfold thickness, and waist circumference for assessing body composition in ambulant and non-ambulant wheelchair games players. Front Physiol 


\section{EXERCISE PHYSIOLOGY}

\section{EP P}

Efficacy of timed and sequential intra-articular injections of linear and of cross-linked hyaluronic acid in patients with knee osteoarthrosis

\author{
E. Barbieri, P. Sestili, G. Annibalini, L. Vallorani, \\ V. Natalucci, S. Contarelli, D. Ligi, L. Croce, V. Stocchi, \\ and I. Capparucci
}

Department of Biomolecular Sciences, University Urbino Carlo Bo, via A. Saffi 2, 61029 Urbino, Italy

Purpose: Knee gonarthrosis (GA) is a leading cause of disabling joint disease, especially in the elderly. The aim of this study was to evaluate the clinical and biochemical effects of timed and sequential intraarticular injections of two different hyaluronic acid (HA) formulations in ameliorating joint pain and function in middle-aged (MA) and elderly (E) patients with knee osteoarthrosis. The effect on cytokine profile and cartilage degradation in plasma and synovial fluid (SF) was studied.

Methods: 39 GA patients (radiographic Kellgren stage II-III) with or without knee effusion were assigned to two groups (19 MA patients aged 50-65 years vs $20 \mathrm{E}>65$ years) according to their age. HA viscosupplementation regimen consisted in a first injection with RegenFlex Starter (32 mg/2 ml MW $8001200 \mathrm{kDa}$, T0) and a second one after 8 days with RegenFlex BIOPLUS $(75 \mathrm{mg} / 3 \mathrm{ml} \mathrm{MW}$ $1 \mathrm{M}+2 \mathrm{M}$ and $500 \mathrm{kDa}, \mathrm{T} 1)$. The same treatment was repeated after 180 days from T0. Clinical visits were scheduled at 3, 6, 9, and 12 months. Cartilage thickness was measured ultrasonographically. Blood and SF (when available) were collected after 8 days (T1) and 3 months (T2) from the first treatment (T0), and analysed for collagen telopeptide II (CTX-II LifeSpan, Inc.) and human cytokines (27 Bioplex Bio-Rad Laboratories) content in plasma and SF, where available. Proteomic approach to detect protein expression variation in SF during 12 months of HA treatment was also used.

Results: Clinical data obtained over the entire 12 months period, shows that viscosupplementation with sequential RegenFlex Starter and Bioplus HA significantly improved knee function and pain scores in both MA and E, independently from BMI, gender, and level of physical activity. Biochemical data indicates that this HA regimen promoted an antinflammatory response paralleled by a coherent and time-dependent modulation of specific cytokines, and induced chondroprotective effects in subjects with grade II arthropathy. Patients in the lowest quartile of CTX-II levels at T2 experienced a mean increase in their medial cartilage thickness. Proteomic approach in SF post HA treatment revealed that proteins associated with inflammation such as Haptoglobin $\alpha 1$ antitrypsin decreased, while Transthyretin, an inhibitor of IL 1, increased.

Conclusions: RegenFlex Starter and Bioplus HA injections to treat knee SF arthropathy significantly ameliorated joint pain score limiting disease progression, especially in low degree chondropathy.

\section{EP P}

Physiological changes induced by 8 weeks whole body eletromyostimulation superimposed to voluntary activation with a load cell

\author{
L. Calanni ${ }^{1}$, M. Vella $^{1}$, D. Greco ${ }^{1}$, L. Castelli $^{1}$ \\ G. Giovanetti ${ }^{1}$, M. Negro ${ }^{1}$, G. D'Antona ${ }^{1,2}$
}

${ }^{1}$ Sport Medicine Centre Voghera, University of Pavia, Pavia, Italy; ${ }^{2}$ Department of Public Health, Experimental and Forensic medicine, University of Pavia, Italy

Purpose: We evaluated the effects of 8 weeks Whole-Body electromyostimyulation (EMS) training with a load cell on physiological parameters and body composition in healthy young subjects.

Methods: 9 healthy active males $(24 \pm 6$ years $)$ and 11 healthy active females ( $22 \pm 9$ years) were trained with a standardized protocol (16 min once a week for 8 weeks) including Whole-Body EMS superimposed (stimulation parameters: rectangular impulse, $80 / 85 \mathrm{~Hz}, 350 \mu \mathrm{s}$, duty ratio $50 \%$ ) to voluntary muscle activation (extensor and flexors muscles; gluteus; lumbar, lower back and upper back; abdominal; pectoral muscles; biceps and triceps) with a load cell. The following physiological parameters were measured before and after training: maximal voluntary contraction (MVC), energy expenditure (EEkc kcal), metabolic rate RMR kcal/day), blood lactate kinetics before (basal, 7 min of training, end of training, 4 and 8 min after), borg scale (CR 10) and body composition with bioimpedance. Statistical analysis was performed using paired $t$ test.

Results: Main results include: MVC: right biceps $+17.32 \%$ $(p<0.05)$, left biceps $+15.24 \%(p<0.05)$, right triceps $+24.56 \%$ $(\mathrm{p}<0.05)$, left triceps $+21.30 \%(\mathrm{p}<0.05)$, pectorals $+13.33 \%$ $(\mathrm{p}<0.05)$, dorsal $+14.60 \%(\mathrm{p}<0.05)$; EEkc: $+24.9 \%(\mathrm{p}<0.05)$; RMR $+8.14 \%(\mathrm{p}<0.05)$; end of training blood lactate $+23.02 \%$ $(\mathrm{p}<0.05)$; Fat free mass $+9.42 \%(\mathrm{p}>0.05)$.

Conclusions: 8 weeks of training with standardized Whole-Body EMS training with load cell induces significant improvements in muscle function and energetics.

\section{Reference}

Kemmler W, Weissenfels A, Willert S, Shojaa M, von Stengel S, Filipovic A, Kleinöder H, Berger J, Fröhlich M (2018) Efficacy and safety of low frequency whole-body electromyostimulation (WBEMS) to improve health-related outcomes in non-athletic adults. A systematic review. Front Physiol 9:573. https://doi.org/10.3389/fphys. 2018.00573. eCollection 2018. 


\section{EP P}

\section{Relationship between heart rate variability} and the levels of physical activity

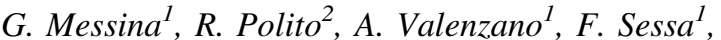 I. A. Triggiani ${ }^{1}$, F. Moscatelli ${ }^{1}$, A. Messina ${ }^{3}$, V. Monda ${ }^{3}$, G. Cibelli ${ }^{1}$, M. Monda ${ }^{3}$, A. Daniele ${ }^{2}$}

${ }^{1}$ Dipartimento di Medicina Clinica e Sperimentale, Università degli Studi di Foggia, Via A.Gramsci 89/91, Foggia, Italy;

${ }^{2}$ Dipartimento di Scienze e Tecnologie Ambientali Biologiche Farmaceutiche, Università degli Studi della Campania, "Luigi Vanvitelli", Via G. Vivaldi 42, 81100 Caserta, Italy;

${ }^{3}$ Dipartimento di Medicina Sperimentale, Sezione di Fisiologia Umana e Unità di Dietetica e Medicina dello Sport, Università degli Studi della Campania, "Luigi Vanvitelli", Via Santa Maria di Costantinopoli, 16, 80138 Napoli, Italy

Purpose: The aim of this study is to compare heart rate variability (HRV) variation in female subjects carrying out different types of physical activity (the physical activity levels were evaluated with the short IPAQ questionnaire) and to demonstrate a relationship between self-regulation, body weight, and physical activity.

Methods: The study was conducted on a cohort of sedentary women $(\mathrm{n}=21)$. Subjects were subdivided into three groups $(\mathrm{n}=7$ for each group): (1) women with BMI of $23.4 \pm 0.7 \mathrm{~kg} / \mathrm{m}^{2}$ who performed walking activities, (2) women with BMI of $21.9 \pm 1.1 \mathrm{~kg} / \mathrm{m}^{2}$ who had moderate activity, (3) women with BMI of $20.2 \pm 0.8 \mathrm{~kg} / \mathrm{m}^{2}$ who performed intense activity.

Results: This study establishes a physical activity role in vegetative modulation in female high fertile age subjects.

Conclusions: Certainly, a reduction in sympathetic activity may be related to lower energy expenditure, thus a reduced energy cost could explain the greater body weight in women who only walk rather than in the other two groups.

\section{EP P}

Muscle stretching does not affect endurance running performance in recreational runners but it decreases the perception of effort

\author{
M. Panascì ${ }^{1,2}$, V. Ferrando ${ }^{1,2}$, A. La Torre ${ }^{3}$, E. Faelli, ${ }^{1,2}$, \\ M. Bove ${ }^{1,2}$, P. Ruggeri ${ }^{1,2}$
}

\footnotetext{
${ }^{1}$ Department of Experimental Medicine, Section of Human Physiology, University of Genoa, Genoa, Italy;

${ }^{2}$ Centro Polifunzionale di Scienze Motorie, University of Genoa, Genoa, Italy;

${ }^{3}$ Department of Biomedical Sciences for Health, University of Milan, Italy
}

Purpose: Contradictory results currently exist on the effects of muscle stretching performed prior to exercise on athletic performance $[1,2]$. This study aimed to investigate the acute effects of static and dynamic stretching incorporated in the warm-up protocol of recreational runners.

Methods: Eight male runners (mean age $36.0 \pm 11.5$ years; body mass $71.99 \pm 9.65 \mathrm{~kg}$; height $176.68 \pm 6.36 \mathrm{~cm}, \quad \mathrm{VO}_{2} \max$ $50.13 \pm 5.12 \mathrm{ml} \mathrm{kg}^{-1} \mathrm{~min}^{-1}$ ) completed, in randomized order, two stretching conditions, static (SS) and dynamic stretching (DS), and a

non-stretching control condition (NS), each condition being performed over three testing sessions, separated by a minimum of $72 \mathrm{~h}$. The endurance running performances were evaluated by time to exhaustion (TTE) and total running distance (TRD), during a treadmill run at maximal aerobic speed. $\mathrm{VO}_{2}$ and blood lactate accumulation during running were measured as indexes of running economy. Rate of perceived exertion (RPE) was examined to express the subjective level of fatigue experienced at the end of each testing session.

Results: No significant effects of the two stretch condition on test performances were demonstrated. Indeed, in SS, DS and NS conditions, TTE measures were $161.63 \pm 42.16 \mathrm{~s}, 166.63 \pm 45.00 \mathrm{~s}$ and $164.38 \pm 36.43 \mathrm{~s}$, respectively, and TRD measures were $666.88 \pm 171.84 \mathrm{~m}, 676.38 \pm 163.16 \mathrm{~m}$, and $669.88 \pm 141.84 \mathrm{~m}$, respectively. $\mathrm{VO}_{2} \max$ (SS: $50.31 \pm 4.96$; DS: $52.74 \pm 6.40$; NS: $51.76 \pm 6.79 \mathrm{ml} \mathrm{kg}^{-1} \mathrm{~min}^{-1}$ ), blood lactate accumulation (DS: $15.18 \pm 2.79$; SS: $15.09 \pm 2.43$; NS: $14.22 \pm 3.32 \mathrm{mmol}^{-1}$ ) and maximal HR (SS: $176.63 \pm 8.75$; DS: $177.63 \pm 10.47$; NS: $176.50 \pm 10.94 \mathrm{bpm}$ ) showed no significant changes between the two experimental conditions and controls. The rate of perceived exertion (RPE) measured after TTE was significantly lower $(\mathrm{p}<0.05)$ in SS and DS conditions (SS: $5.75 \pm 1.75$; DS: $5.50 \pm 1.77$ ) compared with non-stretching control condition $(8.13 \pm 0.99)$.

Conclusions: Under our experimental conditions, muscle stretching does not affect both the endurance running performance and the running economy in recreational runners. However, the reduction of RPE after stretching exercises demonstrates that stretching induced a lower perception of effort during the running performance. Indeed, participants felt to perform better when stretching was incorporated in the warm-up, irrespective of stretch type.

References

Yamaguchi T et al (2015) J Strength Cond Res 29:3045-3052 Blazevich AJ et al (2018) Med Sci Sport Exerc 50:1258-1266

\section{PHYSICAL EXERCISE AS PREVENTION AND THERAPY}

\section{EH P}

\section{Detraining-related changes in left ventricular wall thickness in a young athlete likely to have hypertrophic cardiomyopathy}

\section{R. Restuccial, A. Bonaiuto ${ }^{1}$, F. Trimarchi, ${ }^{1,2}$, L. Magaudda ${ }^{1,2}$, C de Gregorio ${ }^{2,3}$}

${ }^{1}$ Dep. of Biomedical, Dental Sciences and Morphological and Functional Images, Postgraduate School of Sports Medicine. University of Messina, Messina, Italy.

${ }^{2}$ AOU Policlinico "G. Martino", Sports Medicine Unit, Messina, Italy. ${ }^{3}$ Department of Clinical and Experimental Medicine. Cardiology Unit, University Hospital of Messina, Messina, Italy

Purpose: Differential diagnosis between athlete's heart and hypertrophic cardiomyopathy (HCM) in young athletes can be challenging, when electrocardiographic and echocardiographic findings fit into the grey area of left ventricular hypertrophy $(\mathrm{LVH})$. Pre-participation screening programs are important in this clinical setting and some diagnostic criteria may help physicians to discriminate between 
benign and pathological hypertrophy. Literature data indicate that regression of LV wall thickness after detraining for at least 3 months is a possible marker of athlete's heart. We present a controversial case report.

Methods: In the context of a population of young athletes (aged between 15 and 18 years old) we studied some young males with grey-area LVH. In particular, a 16-year-old football player with electrocardiographic and echocardiographic findings suggestive of (apical) hypertrophic cardiomyopathy (wall thickness $15.6 \mathrm{~mm}$ ) was required to withdraw their training session for a three-month period. Results: Detraining resulted in a reduction in the apical wall thickness of about 14\%, and an improvement in ECG repolarization, likely supporting diagnosis of athlete's heart. Our indications were not to continue with competitive sports for another 3 months, but the boy resumed (albeit less intense) their training sessions, and he came back 8 months later for a check-up. Both ECG and echocardiographic findings worsened again.

Conclusions: Evidence from the present report confirms the pathophysiological complexity of LVH in some young athletes classified as to be in the grey area of LVH. It is likely that LV wall thickness may change with detraining also in such clinical setting.

\section{EH P}

\section{Oxygen uptake efficiency slope (OUES) in complex congenital heart disease}

\section{Fugazzotto ${ }^{1}$, R. Tripodi ${ }^{1}$, R. Restuccia ${ }^{2}$, M.F. Panzera ${ }^{2}$, L. Magaudda ${ }^{2}$, F. Trimarchi ${ }^{2}$, D. Di Mauro ${ }^{2}$ \\ ${ }^{1}$ Istituto Clinico COT, Dip. Medicina Interna e riabilitazione cardio vascolare, Messina: \\ ${ }^{2}$ Department of Biomedical, Dental Sciences and Morphological and Functional Images, Postgraduate School of Sports Medicine. \\ University of Messina, Messina, Italy.}

Purpose: Oxygen uptake efficiency slope is a new index of cardiorespiratory functional reserve derived from the logarithmic relation between oxygen uptake (VO2) and minute ventilation during incremental exercise. The oxygen uptake efficiency slope represents how effectively oxygen is extracted and taken into the body from the air, and then ventilated. The OUES is endowed with several clinically useful advantages that other indices of exercise tolerance do not have. First, calculation of the OUES does not need maximal exercise of the subjects. In the literature, few works exist on the use of the OUES as a parameter to evaluate stress tolerance in complex congenital heart disease. The goal of our study was to compare the peak OUES at VO2 in patients suffering from complex congenital heart disease (both young and GUC)

Methods: Twenty congenital complex heart disease patients were enrolled and performed the cardiopulmonary test with calculation of the OUES and peak VO2, with ramp test and breath-by-breath method.

Results: The OUES reflects the peak VO2 in the totality of the patients as happens with other cardiovascular diseases (OUES $1100 \pm 250$, VO2 peak $20 \pm 4 \mathrm{ml} / \mathrm{kg} / \mathrm{min}$ ) in which there is a reduction in tolerance at the effort.

Conclusions: In our opinion, therefore, it can be used as a parameter for exercise tolerance in patients with congenital complex heart disease

\section{EH P}

An integrated program of exercise and psychologicalnutritional support to improve obesity surgery outcomes: results from a 1-year follow-up

\author{
F. Gallè ${ }^{l}$, M. Battaglini ${ }^{2}$, A. Cirella $^{1}$, M. Di Dio ${ }^{1}$, \\ S. Forte ${ }^{1}$, A. Miele ${ }^{1}$, N. Postiglione ${ }^{1}$, R. Ricchiuti ${ }^{1}$, \\ M. Rice ${ }^{2}$, P. Maida ${ }^{2}$, G. Liguori ${ }^{1}$ \\ ${ }^{1}$ University of Naples Parthenope, Naples; \\ ${ }^{2}$ Hospital "Villa Betania", Naples
}

Purpose: Bariatric surgery represents an effective solution for severe obesity; however, a great number of interventions fail due to the unhealthy lifestyles maintained by patients after the surgical treatment. In order to improve surgery outcomes, patients should be accompanied in the post-intervention period through educational and motivational pathways aimed to address their attitudes and behaviors regarding diet and physical activity.

Methods: The Department of Movement Sciences and Wellbeing of the University Parthenope and the Hospital "Villa Betania" in Naples carried out a 1-year intervention based on adapted physical activity and psychological-nutritional support to enhance the adoption of healthy lifestyles in bariatric patients. The intervention group $(\mathrm{n}=10)$ took part to monthly sessions of nutritional education, bi-monthly motivational sessions and bi-weekly exercise sessions; the control group $(n=17)$ received treatment as usual. Both groups were evaluated before and at the end of the intervention through questionnaires investigating eating behaviors and by analyzing their weight and postural status, anthropometric features (waist and hip circumference), and physical fitness (aerobic capacity, strength, flexibility). These evaluations were repeated at follow-up after 1 year since the end of the intervention.

Results: The percentage of total weight loss was higher in the intervention group than in the control group at follow-up. All the physical and behavioral parameters improved significantly among participants to the intervention, who were still active at follow-up, while controls did not show similar results.

Conclusions: A multidisciplinary motivational intervention based on physical activity and nutritional education may be useful to enhance bariatric patients' awareness about the role of lifestyles in improving surgery outcomes.

\section{Reference}

Westerveld D, Yang D (2016) Through thick and thin: identifying barriers to bariatric surgery, weight loss maintenance, and tailoring obesity treatment for the future. Surg Res Pract 2016:8616581

Keywords: Obesity, Bariatric surgery, Multidisciplinary intervention, Lifestyles, Physical activity, Diet 


\section{EH P}

\section{Exercise prescription in breast cancer survivors: adjuvant therapy reduce fat mass loss}

\author{
G. Mascherini, B. Tosi, C. Giannelli, P.A. Modesti, \\ G. Galanti
}

Unità di Medicina dello Sport e dell'Esercizio, Dipartimento di Medicina Sperimentale e Clinica, Università degli Studi di Firenze

Purpose: The efficacy of physical exercise prescription as therapy in breast cancer survivors is largely documented in literature. Unsupervised exercise produces short-term improvements in physical fitness of breast cancer survivors, but regarding the mid-term effectiveness only few studies are available. The purpose of this study was to assess the effects of an unsupervised exercise prescription program on body composition, physical fitness and Health Related Quality of Life of breast cancer survivors.

Methods: Forty-two (average age $52.0 \pm 10.1$ years) women were enrolled.

Assessments performed at baseline and after 6 months of exercise prescription:

- body composition (anthropometric parameters and bioimpedance analysis);

-physical fitness: aerobic capacity by Six-Minute Walk Test (6 MWT), limbs strength by Hand Grip Test and Chair Stand Test, flexibility by Sit and Reach Test;

-Health Related Quality of Life (SF-36).

Statistical analysis was conducted by Student's $t$ tests and multiple regression.

Results: Body composition improvements:

- BMI $\left(\mathrm{T} 0=27.3 \pm 4.2 ; \mathrm{T} 5=26.1 \pm 3.9 \mathrm{~kg} / \mathrm{m}^{2} ; \mathrm{p}<0.001\right)$;

-waist circumference $(\mathrm{T} 0=90.2 \pm 10.8 ; \quad \mathrm{T} 5=85.3 \pm 9.8 \mathrm{~cm}$; $\mathrm{p}<0.001)$

•extracellular $\quad$ water $\quad(\mathrm{T} 0=17.5 \pm 1.9 ; \quad \mathrm{T} 5=16.8 \pm 1.9 \quad \mathrm{~L}$; $\mathrm{p}<0.01)$

$\bullet$ fat mass $(\mathrm{T} 0=25.0 \pm 8.1 ; \mathrm{T} 5=22.6 \pm 7.2 \mathrm{~kg} ; \mathrm{p}<0.001)$

Physical fitness improvements:

- 6 MWT $\quad(\mathrm{T} 0=518.6 \pm 133.0 ; \quad$ T5 $=584.8 \pm 97.2 \mathrm{~m}$; $\mathrm{p}<0.001)$

•Hand Grip $(\mathrm{T} 0=24.3 \pm 4.8 ; \mathrm{T} 5=26.5 \pm 4.5 \mathrm{~kg} ; \mathrm{p}<0.01)$;

-Chair Test $\quad(\mathrm{T} 0=14.5 \pm 3.8 ; \quad \mathrm{T} 5=18.3 \pm 4.3 \quad$ repetitions; $\mathrm{p}<0.001)$

-Sit and Reach $(\mathrm{T} 0=2.6 \pm 9.3 ; \mathrm{T} 5=8.5 \pm 7.1 \mathrm{~cm} ; \mathrm{p}<0.001)$. Health Related Quality of Life improvements:
- Physical Functioning $(\mathrm{T} 0=72.7 \pm 24.6 ; \quad \mathrm{T} 5=83.7 \pm 17.1 \%$; $\mathrm{p}<0.001$ )

•General Health $\quad(\mathrm{T} 0=64.7 \pm 20.4 ; \quad \mathrm{T} 5=69.1 \pm 18.9 \%$; $\mathrm{p}<0.001)$

-Social $\quad$ Functioning $\quad(\mathrm{T} 0=60.5 \pm 24.5 ; \quad \mathrm{T} 5=67.6 \pm 22.9 \%$ $\mathrm{p}<0.05$ )

$\bullet$ Mental Health (T0 = $63.4 \pm 14.8$; T5 = $67.3 \pm 12.5 \%$; $\mathrm{p}<0.05)$.

The percentage change in fat mass has been associated with adjuvant cancer therapy (intercept $=-0.016 ; \mathrm{b}=8.629 ; \mathrm{p}<0.05$ ). Conclusion: An unsupervised exercise prescription program improves body composition, physical fitness and Health Related Quality of Life in breast cancer survivors. Longer term follow-up studies to establish the real capacity of this program to induce longterm changes in lifestyle are needed

References

Azrad M, Demark-Wahnefried W (2014) The association between adiposity and breast cancer recurrence and survival: a review of the recent literature. Curr Nutr Rep 3(1):9-15

Lahart IM, Metsios GS, Nevill AM, Carmichael AR (2018) Physical activity for women with breast cancer after adjuvant therapy. Cochrane Database Syst Rev 1:CD011292. https://doi.org/10.1002/14651858.cd011292.pub2

\section{EH P}

\section{Educational physical activity camp in type 1 diabetes: Can be useful to promote an active lifestyle?}

V. Carnevale Pellino ${ }^{I}$, L. Correale ${ }^{I}$, G. Sorgente ${ }^{I}$, E. Ricagno ${ }^{I}$, S. Dell'Anna ${ }^{1}$, G. Liberali ${ }^{1}$, L. Martinis ${ }^{l}$, O. Ferraro ${ }^{2}$, M. Vandoni ${ }^{1}$

${ }^{1}$ Laboratory of Adapted Motor Activity (LAMA), Department of Public Health, Experimental Medicine and Forensic Science, University of Pavia, Pavia, Italy;

${ }^{2}$ Unit of Biostatistics and Clinical Epidemiology, Department of Public Health, Experimental and Forensic Medicine, University of Pavia, Pavia, Italy

Purpose: Diabetes is a chronic metabolic disease caused by changes in glycemic homeostasis. Physical activity (PA) improves glycemic control and reduces the risk of chronic diabetes-related complications. Despite this evidence, most people with type 1 diabetes do not regularly exercise, mainly due to fear of hypoglycemia during exercise. Improving knowledge of patients in the management of the pathology during exercise can help them to reduce factors that limit PA. The aim of this study was to evaluate the role of an educational physical activity camp for patients with type 1 diabetes in improving active lifestyle.

Methods: 26 subjects (12 females, $41.5 \pm 11.49$ years) with type 1 diabetes attended in an educational camp focused on PA and management of glycaemia before, during and after exercise. All subjects filled three questionnaires: "IPAQ" that investigates individual level of PA, "PAID" that analyzes individual problems of the disease and "EMI" that examines the different motivations for PA practice. The questionnaires were filled before and at the end of the camp, then after 1,3 and 6 months. The scores of the questionnaires were analyzed by the Mann-Whitney $U$ test for independent data. Values statistically significant were analyzed out using the STATA ${ }^{\circledR}$ v14 software.

Results: One month from the end of the camp, IPAQ shows that males increased significantly their level of PA $(p<0.05)$ and for both gender, improvements continued over 3 and 6 months. Subjects who had higher values of PAID before the camp presented lower levels of 
PA. Practice of PA increased similarly for subjects with a lower and higher score of PAID before the camp. At least, the EMI's results show that for both gender achieving of "physical well-being" is the most important motivation to increase PA level.

Conclusions: The current findings showed a positive impact of educational camp on improvement of PA level. A better knowledge in diabetes management during exercise could lead subjects to a more active lifestyle reducing limitation factors like fear of hypoglycaemia. The achievement of "physical well-being" could be useful to improve the motivation and increase PA level. Finally, our results suggest that PA promotion should be better focused on woman and patients with a greater difficulties perception.

\section{References}

Farinha JB, Krause M, Rodrigues KJ, Reischak OA (2017) Exercise for type 1 diabetes mellitus management: general considerations and new directions. Med Hypotheses 104:147-153

Souto DL, Demiranda MP (2011) Physical exercises on glycemic control in type 1 diabetes mellitus. Nutricion Hospitalaria 26:425-429

\section{EH P \\ Effects of exercise on breast cancer triple-negative cell proliferation}

\author{
V. Natalucci, G. Baldelli, M. Gervasi, M. De Santi, \\ G. Annibalini, F. Lucertini, G. Brandi, V. Stocchi, \\ P. Sestili, E. Barbieri \& L. Vallorani
}

Department of Biomolecular Sciences, Division of Exercise and Health Sciences, University of Urbino Carlo Bo, Urbino, Italy

Purpose: The purpose of this study was to examine if systemic responses to acute endurance exercise pre and post-9 weeks of training in sedentary healthy women could modulate TNBC cell proliferation in vitro.

Methods: 12 sedentary healthy women (age $21 \pm 0.8$ years, high $160 \pm 4.7 \mathrm{~cm}$, weight $53 \pm 5.2 \mathrm{~kg}$, BMI $21 \pm 1.2$ ), performed 36 indoor cycling training sessions over a 9-weeks period. Before (pre) and after (post) this training protocol, subjects were asked to perform a high-intensity-interval endurance cycling test (HIIT). HIIT consisting in a 20 min warm up composed by four 5 min steps at 50,60 , 65 and $70 \%$ of the individual maximal power (Pmax), previously determined by the incremental test. After warm up, subjects performed ten $90 \mathrm{~s}$ sprints ("SPR" steps) at $90 \%$ Pmax separated by 3 min recovery intervals ("REC" steps) at 55\% Pmax. At the end of the last REC step subjects performed a 11th SPR step until the exhaustion.

Blood samples were collected pre, immediately afterand 4 and $24 \mathrm{~h}$ post-exercise. Sera obtained were used to supplement culture medium for TNBC MDA-MB-231 cells to evaluate cell proliferation. TNBC cells were seeded 20,000 cells/well in 24 wells. After overnight, culture medium was changed with DMEM red-phenol free with physiological concentration of glucose $(0.8 \mathrm{mg} / \mathrm{mL})$ and conditioned with $5 \%$ of human pre/postexercise serum; after $48 \mathrm{~h}$, cells were counted by hemocytometer and trypan blue exclusion assay.

Results: Sera collected after acute endurance exercise performed pre and post-training induced the same effect on TNBC cell proliferation; in particular $4 \mathrm{~h}$ post-exercise sera, both pre and post-training, led to a statistically significant reduction in proliferation of about $24-25 \%$ compared to cells supplemented with pre-exercise sera (one-way ANOVA followed by Dunnett's Multiple Comparison Test; $\mathrm{p}<0.001)$. The reduction of proliferation resulted from $4 \mathrm{~h}$ postexercise sera was maintained also by $24 \mathrm{~h}$ post-exercise sera.
Conclusions: The systematic responses indicate that acute exercise before or after a training period in sedentary pre-menopausal healthy women might control the cancer cell proliferation and add a positive effect to the overall beneficial influence of exercise on TNBC.

References

ACSM's guidelines (2017) Guidelines for exercise testing and prescription, 10th edn. Lippincott and Wilkins, Philadelphia O'Hara JP et al (2012) Int J Sports Med 33(1):18-25

\section{EH P \\ Rest-activity circadian rhythm in women with metabolic syndrome}

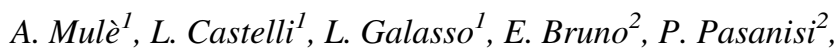
S. Longo ${ }^{1}$, S. Shokohyar ${ }^{1}$, A. Caumo ${ }^{1}$, A. Montaruli, ${ }^{1,3}$, F. Esposito ${ }^{1,3}$, E. Roveda ${ }^{1,3}$

${ }^{1}$ Department of Biomedical Sciences for Health, University of Milan, Milan, Italy;

${ }^{2}$ Department of Preventive and Predictive Medicine, Fondazione IRCCS, Istituto Nazionale Tumori, Milan, Italy;

${ }^{3}$ IRCSS Istituto Ortopedico Galeazzi, Milan, Italy

Purpose: The circadian rhythms produce 24-h oscillations of several physiological variables and any irregularity of these rhythms exposes the subject to an increased risk of metabolic syndrome. The metabolic syndrome is a complex of correlated risk factors such as high blood pressure, abdominal obesity, high fasting glycemia and dyslipidemia. These risk factors expose the subject to cardiovascular diseases and type 2 diabetes mellitus. Furthermore, there is a correlation between circadian rhythms of many variables and metabolic syndrome. Aim of the study was to assess the Rest-Activity circadian Rhythm (RAR) in women with metabolic syndrome in relation to BMI.

Methods: We recruited 49 adult women with metabolic syndrome from Fondazione IRCCS, Istituto Nazionale Tumori. All participants underwent a continuous 7-day actigraphic monitoring to detect the RAR. The participants were divided into three groups referring to their BMI: group 1, with BMI between 25 and $30(\mathrm{n}=18)$; group 2, with BMI between 30 and $35(\mathrm{n}=23)$; group 3 with BMI $>35$ $(\mathrm{n}=8)$. All data were analyzed by single cosinor method to obtain the rest-activity circadian rhythm parameters: MESOR (M), amplitude (A) and acrophase (Ø). Afterwards, we applied the mean cosinor method to the three groups.

Results: We found a statistically significant differences for MESOR (M group $1=269.8$ vs $\mathrm{M}$ group $2=226.9 ; \mathrm{p}<.05$ ) and amplitude (A group $1=212.1$ vs A group $2=171.8 ; \mathrm{p}<.05)$ between group 1 and 2 (ANOVA).

Conclusions: These results show a reduction of MESOR and amplitude in relation to increase of BMI.

References

Sohail et al (2015) Irregular 24-hour activity rhythms and the metabolic syndrome in older adults. Chronobiol Int 32(6):802-813

Chen C-C, Ringenbach SDR (2018) Metabolic syndrome, actigraphy, circadian rhythm, women, body mass index, physical activity levels of Intellectual Disability Research 62(4):339-348. https://doi.org/10.1111/jir.12474 


\section{EH P}

\section{Effect of Baskin on sleep quality in adolescent with Down syndrome}

\author{
L. Castelli ${ }^{1}$, A. Mulè ${ }^{1}$, L. Galasso ${ }^{1}$, G. Merati ${ }^{1}$, M. Algeri ${ }^{1}$, \\ S. Rampichini ${ }^{1,2}$, A. Caumo $^{1}$, A. Montaruli, ${ }^{1,2}$, \\ F. Esposito ${ }^{1,2}$, E. Roveda ${ }^{1,2}$ \\ ${ }^{1}$ Department of Biomedical Sciences for Health, University of Milan, \\ Milan, Italy; \\ ${ }^{2}$ IRCSS Istituto Ortopedico Galeazzi, Milan, Italy
}

Purpose: Baskin is a new discipline included in basketball, with the purpose to allow different people (men and women, younger and elderly, people with or without disabilities) to play together. The aim of this study was to evaluate the sleep quality before and after a Baskin match in individuals with Down syndrome.

Methods: We recruited 10 adolescents (mean age $17.6 \pm 3.2$ years) with Down syndrome, 7 males and 3 females. According to the regulations of Baskin, 8 subjects played in the Role 3, a dynamic player that can run all over the playground, and 2 subjects in the Role 2, a static player called Pivot.

All the participants underwent to an actigraphic monitoring to evaluate the sleep quality during the night before (night-PRE) and the night after (night-POST) the Baskin match. The following sleep parameters were evaluated both for the night-PRE and the nightPOST: Actual Sleep Time-AST, Sleep Efficiency-SE, Sleep LatencySL, Movement and Fragmentation Index-MFI, Immobility Time-IT, and Moving Time-MT

Results: All the participants showed a deterioration of sleep quality during the night-POST. The SE (night-PRE: $85.4 \pm 4.3 \%$; nightPOST: $76.8 \pm 5.4 \% ; \mathrm{p}=0.05$ ), and IT (night-PRE: $85.3 \pm 8.0 \%$; night-POST: $77.4 \pm 5.3 \% ; \mathrm{p}=0.03$ ), representative for restful sleep, decreased. On the contrary, the MFI (night-PRE: $33.7 \pm 8.5$; nightPOST: $51.6 \pm 9.9 ; \mathrm{p}=0.02$ ), and MT (night-PRE: $14.7 \pm 8.0 \%$; night-POST: $22.6 \pm 5.4 \% ; \mathrm{p}=0.03$ ), representative for fragmented sleep, increased.

These results showed a worsening in sleep quality in the night after the Baskin match, both for Role 3 and Role 2.

Conclusions: The reason of this sleep worsening should be more investigated, by assessing also cardiovascular modifications, in terms of the sympathetic-vagal balance.

\section{References}

Chen CJJ, Ringenbach SDR (2018) Walking performance in adolescents and young adults with Down syndrome: the role of obesity and sleep problems. J Intellect Disalbil Res 62(4):339-348.

Baskin Association regulations. https://baskin.it/baskin-a-sportfor-everyone

\section{EH P \\ Circadian rhythm of activity levels in breast cancer women}

\author{
L. Galasso ${ }^{1}$, L. Castelli ${ }^{1}$, A. Mulè ${ }^{1}$, E. Bruno ${ }^{2}$, P. Pasanisi ${ }^{2}$, \\ E. Cé $e^{1,3}$, C. Doria ${ }^{l}$, A. Caumo ${ }^{1}$, A. Montaruli ${ }^{1,3}$, \\ F. Esposito ${ }^{1,3}$, E. Roveda ${ }^{1,3}$ \\ ${ }^{1}$ Department of Biomedical Sciences for Health, University of Milan, \\ Milan, Italy; \\ ${ }^{2}$ IRCSS Istituto Nazionale Tumori, Milan, Italy; ${ }^{3}$ IRCCS Istituto \\ Ortopedico Galeazzi, Milan, Italy
}

Purpose: Rest-activity circadian rhythm (RAR), which is the expression of the locomotor activity level in the $24 \mathrm{~h}$, is used as a marker of the circadian timing system (CTS). Many studies showed the presence of a bidirectional link between RAR and cancer: a disrupted RAR have been associated with higher cancer incidence; on the other hand, cancer progression was accelerated in patients with an altered RAR. The aim of this study was to analyze RAR in a population of breast cancer (BC) survivors, 5 years after diagnosis, compared to healthy individuals.

Methods: A total of 28 women volunteered to be part of the study. Fifteen of them were patients with BC diagnosis (BC-Group) and other thirteen participants acted as sex-, age-, and BMI-matched controls (Ctrl). All 28 participants were subjected to the following detections: height and body mass to calculate BMI; systolic and diastolic blood pressure; heart rate by an electronic sphygmomanometer; glycemia, total cholesterol, high density lipoproteins, low density lipoproteins and triglycerides; continuous 5-day actigraphic monitoring (during week days) to detected the RAR.

Results: The anthropometric, cardiovascular and blood chemistry characteristics were homogeneous in BC-Group and Ctrl. No significant differences were observed between the two groups for all parameters. The single cosinor method revealed statistically significant RAR $(\mathrm{p}<0.001)$ in all participants. The population mean cosinor applied to BC-Goup and Ctrl revealed the presence of a significant circadian rhythm in both groups $(\mathrm{p}<0.001)$. MESOR and amplitude resulted significantly different between the two groups: MESOR (194.6 vs 276.4 a.c. in BC-Group and Ctrl, respectively; p < .001) and amplitude (157.9 vs 222.6 a.c.in BC-Group and Ctrl, respectively; $\mathrm{p}<.001$ ) was significantly lower in BC-Group compared to Ctrl.

Conclusions: The women with $\mathrm{BC}$, at 5 years after diagnosis, showed lower activity levels compared to healthy controls.

Reference

Berger AM, Hertzog M, Geary CR, Fischer P, Farr L (2012) Circadian rhythms, symptoms, physical functioning, and body mass index in breast cancer survivors. J Cancer Surviv 6:305-314 


\section{EH P}

Leisure-time physical activity for health promotion in elderly: initial evidence from the "Passiata Day" model

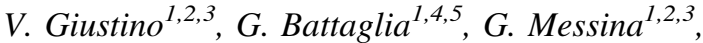 \\ M. Faraone ${ }^{6}, J$. Brusa $^{1,2,3}$, L. Martorana ${ }^{1}$, A. Bordonali ${ }^{6}$, \\ L.J. Domínguez ${ }^{6}$, M. Barbagallo ${ }^{6}$, A. Palma $^{1,4,5}$
}

${ }^{1}$ Department of Psychological, Pedagogical and Educational Sciences, University of Palermo, Palermo, Italy;

${ }^{2}$ Posturology and Biomechanics Laboratory Research Unit, University of Palermo, Palermo, Italy;

${ }^{3}$ Posturalab Italia Research Institute, Palermo, Italy;

${ }^{4}$ Sport and Exercise Sciences Research Unit, University of Palermo, Palermo, Italy;

${ }^{5}$ Regional Sports School of CONI Sicilia, Sicily, Italy;

${ }^{6}$ Geriatric Unit, Department of Internal Medicine and Geriatrics, University of Palermo, Palermo, Italy

Purpose: Falls prevention interventions are a priority for public health systems in order to reduce falls-related high costs. The University Hospital of Palermo promoted the "Passiata Day" model, an initiative of the Department of Geriatrics that provided a 1-h walk for once a week in a park of Palermo. It was guided by a graduate in physical education and a medical doctor. The aim of this study was to evaluate posturographic parameters in a sample of elderly including a group of subjects that had been walking regularly in the "Passiata Day" model for 6 months and a group of sedentary elderly subjects. Methods: A number of 109 older people were recruited at the University Hospital of Palermo and divided into the following groups: the physical activity group (PAG: $\mathrm{n}=75$; age $70.87 \pm 7.09$ years; height $160.75 \pm 7.38 \mathrm{~cm}$; weight $70.71 \pm 13.87 \mathrm{~kg}$ ) and the sedentary group (SG: $\mathrm{n}=34$; age $75.5 \pm 9.43$ years; height $157.32 \pm 19.60 \mathrm{~cm}$; weight $70.94 \pm 21.64 \mathrm{~kg}$ ). All participants performed a stabilometric test in two different conditions: with eyes open (EO) and with eyes closed (EC) using the freeMed ${ }^{\circledR}$ baropodometric platform and the freeStep ${ }^{\circledR}$ software (Sensor Medica ${ }^{\circledR}$; Guidonia Montecelio, Roma, Italia). In accordance with the Romberg test, subjects were required to stay in a standing position for $51.2 \mathrm{~s}$ with feet placed side-by-side forming an angle of $30^{\circ}$ and both heels were $4 \mathrm{~cm}$ apart. The parameters considered were: Sway Path Length (SPL) of the CoP; Ellipse Sway Area (ESA); CoP coordinates along the frontal $(\Delta \mathrm{X} ; \mathrm{X}$-mean) and sagittal $(\Delta \mathrm{Y} ; \mathrm{Y}$-mean) planes. Mean values and standard deviations were calculated using Statistica Software 12 (StatSoft ${ }^{\circledR}$, TIBCO $^{\circledR}$ Software Inc, Palo Alto, CA, USA). Differences between groups were analysed via $t$ tests for comparisons. The alpha level was set at $p<0.05$.

Results: The stabilometric evaluation with EO did not show any significant differences $(p>0.05)$ between the PAG and SG for all the posturographic parameters. Instead, as concerns the EC condition, we found a significant difference in $\mathrm{ESA}(p=0.01), \Delta \mathrm{X}(p=0.002), \Delta \mathrm{Y}$ $(p=0.02)$ of the CoP in the PAG compared to the SG.

Conclusions: Our results showed that a leisure-time physical activity improve balance in elderly, in particular in stress conditions as with EC. Indeed, the PAG showed significantly lower postural sway parameters when compared to SG. This indicates that walking regularly can increase proprioceptive capacity in elderly in order to prevent the risk of fall.

Reference

Okubo Y, Osuka Y, Jung S, Rafael F, Tsujimoto T, Aiba T, Kim T, Tanaka K (2016) Walking can be more effective than balance training in fall prevention among community-dwelling older adults. Geriatr Gerontol Int 16(1):118-125

\section{EH P}

Team game for elderly

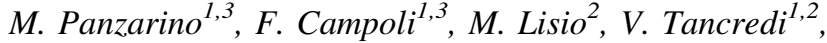 \\ G. Annino ${ }^{1,2}$, E. Padua ${ }^{1,2}$ \\ ${ }^{1}$ Università Telematica San Raffaele, Roma, ItaliaDip. di \\ "Promozione delle scienze umane e della qualità della vita"; \\ ${ }^{2}$ Università di Roma Tor Vergata, Roma, Italia; \\ ${ }^{3}$ Accademia Nazionale di Cultura Sportiva
}

Purpose: To investigate the motor activity program effects in elderly people, based on the practice of an adapted and controlled team game for the elderly: the Skymano and the effects on the improvement and/ or maintenance of the elderly people physical abilities

Methods: 235 subjects ( $70.5 \pm 6.1 \mathrm{anni}), 31 \mathrm{men}$, divided into three groups. An experimental group SG (96 subjects) that practices the sports game skymano, a gymnastic group GG (94 subjects) that practices general gymnastics and a GC control group (44 subjects) that doesn't practice motor activity.

The two gym groups practice activities twice a week $\left(50^{\prime}\right.$ per session) for 8 months. The tests evaluate the flexibility of the chain back muscle, balance, the abdominal, legs and hadgrip strength. The groups, in baseline, are homogeneous in them motor skills analyzed. Results: Descriptive statistical procedures are presented as mean and the percentage changes $(\Delta \%)$ were calculated for each study group $(($ Media Post - Media pre $) /$ Media Pre $) \times 100$. The significance level were performed of $\mathrm{p}<0.05$.

Abdominal and lower limb strength are improved with the same intensity: lower limb strength: GS $\Delta 6 \%(\mathrm{p}<0.01)$ GG $\Delta 5 \%$ $(\mathrm{p}=0.02)$, CG $\Delta-6 \%(\mathrm{p}=0,01) ;$ abdominal strength SG $\Delta 5 \%$ $(\mathrm{p}=0.01)$ GG $\Delta 6 \%(\mathrm{p}=0.04)$, CG $\Delta-2 \%(\mathrm{p}=0.2)$; Flexibility: $\mathrm{SG}$ $\Delta 13 \%(\mathrm{p}=0.1)$ GG $\Delta 29 \%(\mathrm{p}<0.01), \mathrm{CG}-1 \%(\mathrm{p}=0.4)$. Important results are in the handgrip test $\mathrm{SG} \Delta 3 \%(\mathrm{p}=0.02)$; GG $\Delta-1 \%$ $(\mathrm{p}=0.2)$ and CG $\Delta-2 \%(\mathrm{p}=0.2)$, and in balance: SG $\Delta 26 \%$ $(\mathrm{p}=0.02)$ GG $\Delta 16 \%(\mathrm{p}=0.04)$ and CG $\Delta 2 \%(\mathrm{p}=0.4)$.

Conclusions: Both motor protocols have made significant improvements in motor skills in elderly who generally undergoing a physiological decline. The skymano game has detected a gymnastic practice for the balance and the handgrip strength ensuring fun and socialization in the elderly

\section{Reference}

Cvecka J et al (2015) Physical activity in elderly. Eur J Transl Myol 25(4):249-252 


\section{EH P}

Investigation of the determinants for the adherence to an exercise program in cancer patients: a pilot study

\author{
A. Avancini ${ }^{1}$, V. Pala ${ }^{2}$, V. Krogh ${ }^{2}$, S. Sieri ${ }^{2}$, L. Mariani ${ }^{2}$,

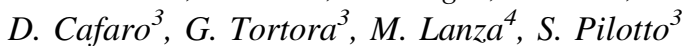

${ }^{1}$ Clinical and Experimental, Biomedical Sciences, Department of Medicine, University of Verona, Italy;

${ }^{2}$ Epidemiology and Prevention Unit, Fondazione IRCCS Istituto Nazionale dei Tumori, Milan, Italy;

${ }^{3}$ Medical Oncology, University of Verona, Azienda Ospedaliera

Universitaria Integrata, Verona, Italy;

${ }^{4}$ Department of Neurosciences, Biomedicine and Movement Sciences, University of Verona, Italy

Purpose: Physical exercise (EX) provides benefits for cancer survivors, such as improvement of quality of life, cancer-related fatigue, health-related skills [1]. Despite the evidences, most cancer survivors are sedentary or insufficiently active [2]. The purpose of this study is to provide an assessment of EX preferences in cancer survivors.

Methods: A questionnaire to assess EX preferences, physical activity (PA), demographic and health information, was developed for use in cancer survivors. A representative sample of patients from the Medical Oncology Unit of the Verona Hospital were asked to anonymously complete the questionnaire in April-June 2018. PA behavior was assessed by leisure score index (LSI) from the validated Godin's Leisure Time Exercise Questionnaire (GLTEQ) [3]. EX preferences questions were drawn from Jones and Courneya questionnaire [4].

Results: The percentage of patients that resulted sufficiently active, with a LSI $>23$, was low, only $7 \%$. More than half of the patients $(51 \%)$ expressed strong interest to start a physical exercise program. Another 29\% expressed less stronger but still interested in participating in a EX program.

Regarding the preferred source of PA information, "the oncologist" was the preferred category (49\%) followed by physiotherapist $(27 \%)$. The preferred way of information delivery was "face-to-face" $(77 \%)$ followed by no particular preferences (14\%).

Working out in groups was preferred by $42 \%$ of patients, whereas $26 \%$ choose an individually supervised home program. The preferred composition of EX group was with "other cancer survivors" $(27 \%)$. The favorite place to exercise was outdoors $(32 \%)$ and a fitness center for adapted physical activity (28\%). Two times/week (45\%), and three times/week $(31 \%)$ were the preferred EX frequencies. "Mild" was the preferred EX intensity (56\%), followed by "moderate" (39\%).

Conclusion: Very few cancer survivors were found sufficiently active, but about $80 \%$ were willing to start EX programs. This preliminary results encourages intervention studies to improve PA in cancer survivors offering multiple options based on patient's EX preferences.

References

1. Schmitz KH et al (2010) Med Sci Sports Exerc 42:1409-1426

2. McGowan EL et al (2013) Oncol Nurs Forum 40(1):44-52

3. Amireault $\mathrm{S}$ et al (2015) BMC Med Res Methodol 15(1)

4. Jones LW, Courneya KS (2002) Cancer 10(4):208-215

\section{EH P}

\section{Comparative analyses of Short-term High Intensity Interval Training vs Steady State Training protocols in overweight sedentary subjects}

\author{
A. Mancini ${ }^{1,2}$, L. Russomando ${ }^{1}$, V. Bono ${ }^{1}$, S. Orrù ${ }^{1,3}$, \\ E. Imperlini ${ }^{3}$, P. Buono ${ }^{1,2,3}$ \\ ${ }^{1}$ Department of Movement Sciences and Wellness, University \\ Parthenope, Naples, Italy; \\ ${ }^{2}$ CEINGE-Biotecnologie avanzate, Napoli, Italy; \\ ${ }^{3}$ IRCCS SDN Foundation, Naples, Italy
}

Purpose: Obesity is one of the biggest risk factors for health. The American College of Sports and Medicine (ACSM) recommends $150 \mathrm{~min}$ of moderate or $60 \mathrm{~min}$ of intense daily exercise in order to counteract the fat accumulation (1). The aim of the present study was to investigate the effects of a short-term High Intensity Interval Training (sHIIT) protocol compared to the classical Steady State Training (SST) on weight-loss, on body composition and on regular physical exercise compliance.

Methods: 18 sedentary subjects $(18-30$ years; $25 \geq$ BMI $\leq 30)$ were randomized into 2 groups: 8 subjects ( 3 males and 5 females) were assigned to the sHIIT group and 10 subjects ( 6 males and 4 females) were assigned to the SST group; 5-min warm up and 20-min resistance training (leg press, lat machine, chest press, crunch base$3 \times 12,60-70 \% 1-\mathrm{RM}$, each) were common to both training protocols.

SHIIT protocol: the cardiovascular training session $(8 \mathrm{~min})$ was divided into 4 stages; each stage had $30 \mathrm{~s}$ at $140 \%$ of the maximum intensity reached in the incremental test (HR $\geq 90 \%$ HRmax) and $90 \mathrm{~s}$ of active recovery, performed at $25 \%$ of the maximum intensity reached in the incremental test. SST protocol: the continuous cardiovascular training session $(30 \mathrm{~min}$ ) was performed at $60-70 \%$ of the predicted theoretical HRmax. Body composition (Girth Method), VO2 max (Single Stage Treadmill Walking test) and 1-RM were determined at T0 (baseline) and T1 (6 weeks) for all participants.

Results: After 6 weeks of sHIIT and SST training, a decrease in BMI $(p<0.001), \%$ fat mass $(p<0.05)$ and abdominal circumference $(\mathrm{p}<0.05)$ and an improvement of VO2max $(\mathrm{p}<0.001)$ were observed in all exercised subjects with no significant differences

Table 1 Analysis of randomized controlled trials on physical activity in advanced lung cancer

\begin{tabular}{|c|c|c|c|c|}
\hline Authors & Sample & Design & Exercise intervention & Results \\
\hline $\begin{array}{r}\text { HENKE CC et al., } \\
\text { Support Care } \\
\text { Cancer } 2014\end{array}$ & $\begin{array}{l}46 \text { advanced-stage } \\
\text { lung cancer } \\
\text { patients } \\
\text { receiving } \\
\text { chemotherapy; }\end{array}$ & $\begin{array}{l}\text { Conventional } \\
\text { physiotherapy vs } \\
\text { special } \\
\text { physiotherapeutic } \\
\text { training } 29 \text { pts } \\
\text { completed trial (18 in } \\
\text { experimental group) }\end{array}$ & $\begin{array}{l}\text { Implementing daily endurance } \\
\text { and strength training }\end{array}$ & $\begin{array}{l}\text { Significant improvements } \\
\text { in physical function } \\
\text { scores, self-reported } \\
\text { symptoms, and } \\
\text { exercise tolerance }\end{array}$ \\
\hline $\begin{array}{c}\text { CHEN MH et al., } B r \\
\quad J \text { Cancer } 2015\end{array}$ & $\begin{array}{l}116 \text { lung cancer } \\
\text { patients (10 } \\
\text { receiving } \\
\text { systemic } \\
\text { therapy) }\end{array}$ & $\begin{array}{l}\text { Walking exercise group } \\
\qquad \begin{array}{l}(\mathrm{n}=58) \text { or a usual- } \\
\text { care group }(\mathrm{n}=58) \\
26 \text { pts completed } \\
\text { 12-week programme }\end{array}\end{array}$ & $\begin{array}{l}\text { 12-week exercise programme } \\
\text { that comprised home-based, } \\
\text { moderate-intensity walking } \\
\text { for } 40 \text { min per day, } 3 \text { days } \\
\text { per week, and weekly } \\
\text { exercise counselling }\end{array}$ & $\begin{array}{l}\text { Effects of the exercise } \\
\text { programme on } \\
\text { anxiety, depression } \\
\text { and cancer-related } \\
\text { symptoms }\end{array}$ \\
\hline $\begin{array}{l}\text { DHILLON HM et al., } \\
\text { Ann Oncol } 2017\end{array}$ & $\begin{array}{l}112 \text { lung cancer } \\
\text { patients (56 } \\
\text { assigned to each } \\
\text { group) }\end{array}$ & $\begin{array}{l}\text { Usual Care (nutrition and } \\
\text { PA education } \\
\text { materials) or } \\
\text { experimental } \\
\text { intervention }\end{array}$ & $\begin{array}{l}\text { EX: UC plus 2-month supervised } \\
\text { weekly PA and behaviour } \\
\text { change sessions }\end{array}$ & $\begin{array}{c}\text { No difference was seen in } \\
\text { fatigue or QOL }\end{array}$ \\
\hline
\end{tabular}


between the 2 protocols. The compliance at the end of the study was $2.8 \mathrm{~d}^{*}$ week-1 for sHIIT vs $2.3 \mathrm{~d}^{*}$ week- 1 for SST protocols, respectively. Further, 4 subjects ( $3 \mathrm{~m}$ and 1f) belonging to SST group did not complete the program.

Conclusion: Our preliminary results indicate that both sHIIT and SST protocols gave the same outcome on BMI reduction and body composition; sHIIT protocol resulted in a better compliance to regular exercise program compared to the classical SST protocol.

Acknowledgements: We thanks Palestra ADS Ardor, Naples for the kindly collaboration.

Reference

Donnelly JE et al (2009) Med Sci Sports Exerc 41:459-471

\section{EH P \\ Challenges and promises of physical exercise in advanced lung cancer patients.}

\section{T. Franchina ${ }^{1}$, A. Russo ${ }^{2}$, G.R.R. Ricciardi ${ }^{2}$, M. Righi ${ }^{3}$,

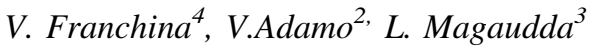

${ }^{1}$ Department of Human Pathology "G. Barresi”; Second cycle degree "Theory and Methods of Preventive and Adapted Physical Activities", University of Messina;

${ }^{2}$ Department of Human Pathology "G. Barresi" and Unit of Medical Oncology, A.O. Papardo, Messina;

${ }^{3}$ Department of Biomedical and Dental Sciences and Morphofunctional Imaging; Second cycle degree "Theory and Methods of Preventive and Adapted Physical Activities", University of Messina;

${ }^{4}$ Unit of Medical Oncology, A.O. Papardo, Messina

Purpose: In the last decades, the benefits of physical exercise for lung cancer patients are widely described in prevention, preoperative and rehabilitation settings.

Physical exercise for advanced stage lung cancer patients is a less explored area.

Exercise training offers great promises in this setting, including improvements in physical functioning and quality of life and contrasting the "vicious circle" of dyspnea.

Recent clinical practice guidelines recommend physical activity for cancer patients, however evidence has not translated into clinical practice in advanced lung cancer.

Methods: A systematic literature review has been conducted to identify specific published randomized controlled trial (RCT) and evaluate the barriers to include physical activity into multidisciplinary management of advanced lung cancer.

Results: Three RCT have been included in the analysis and summarized in Table 1.

Results are promising, but should be viewed cautiously due to small sample sizes and the differences in patient characteristics and treatments. Conclusions: Emerging data underline the effects of physical activity and exercise training on clinical outcomes in advanced lung cancer patients. Several clinical trials are ongoing (i.e. EXHALE and EMBRACE-ClinicalTrials.gov).

\section{EH P}

\section{Influence of physical activity on posturographic parameters and muscular characteristics in children with GH deficiency}

\author{
J. Brusa ${ }^{1,2}$, M.C. Maggio ${ }^{3}$, G. Messina ${ }^{1,2}$, M. Bellafiore ${ }^{4}$,

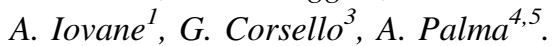 \\ ${ }^{1}$ Department of Psychological, Pedagogical and Educational \\ Sciences, Posturology and Biomechanics Laboratory Research Unit, \\ University of Palermo, Palermo, Italy; \\ ${ }^{2}$ Posturalab Italia Research Institute, Palermo, Italy; \\ ${ }^{3}$ Department Pro.S.A.M.I., University of Palermo, Palermo, Italy; \\ ${ }^{4}$ Department of Psychological, Pedagogical and Educational \\ Sciences, Sport and Exercise Sciences Research Unit, University \\ of Palermo, Palermo, Italy; \\ ${ }^{5}$ Regional Sports School of CONI Sicilia, Sicily, Italy
}

Purpose: Several studies have been assessed children with GH deficiency in exercise testing. It is known that physical activity increases GH secretion. Muscular strength, power and postural characteristics represent health-related fitness characteristics. The purpose of the present study was to investigate the influence of physical activity in GH-deficient young patients on posturographic parameters and muscular characteristics.

Methods: A number of twenty-two (13 males and 9 females) children affected by GH deficiency were recruited in Palermo University Hospital and divided into the physical activity group (PAG: $n=14$; age $14.92 \pm 1.97$ years; height $147.57 \pm 11.86 \mathrm{~cm}$; weight $42 \pm 11.41 \mathrm{~kg}$ ) with $\geq 3$ consecutive years of physical activity background and the sedentary group (SG: $\mathrm{n}=8$; age $16.37 \pm 3.70$ years; height $138 \pm 15.04 \mathrm{~cm}$; weight $38.88 \pm 16.43 \mathrm{~kg}$ ). All subjects completed the protocol assessment that included the following evaluations: a baropodometric test using the freeMed ${ }^{\circledR}$ baropodometric platform and the freeStep ${ }^{\circledR}$ software (Sensor Medica ${ }^{\circledR}$; Guidonia Montecelio, Roma, Italia), a hand grip test through a mechanical dynamometer (Kern Map model 80K1Kern $^{\circledR}$, Kern \& Sohn GmbH, Balingen, Germany) and a Sergeant test. Mean values and standard deviations were calculated using Statistica Software 12 (StatSoft ${ }^{\circledR}$, TIBCO $^{\circledR}$ Software Inc, Palo Alto, CA, USA). Differences between groups were analysed using a $t$ test. The p-value was set at $\mathrm{p}<0.05$.

Results: The baropodometric test showed a significant difference $(p<0.05)$ between PAG and SG for forefoot-rearfoot distribution for both feet. In particular, our data showed higher significant load pressure on right forefoot as well as on left forefoot in PAG compared to SG. For the hand grip test, PAG results showed higher levels statistically significant $(\mathrm{p}<0.05)$ respect to $S G$. As concern the Sergent test, any significant difference was found ( $p>0.05)$. Finally, variable associations were tested by Pearson's coefficient of correlation showing a significant positive correlation between Sergeant test values and right forefoot load pressure in PAG $(\mathrm{r}=0.58, \mathrm{p}<0.05)$. Conclusions: Our results suggest that in GH-deficient children physical activity may induce to higher muscular strength levels and influence baropometric characteristics. Considering the results of this study, we deem necessary to conduct further investigations regarding the relationships among physical exercise and GH deficiency.

\section{Reference}

Zueger T, Alleman S, Christ ER, Stettler C (2011) Exercise-induced GH secretion in the assessment of GH deficiency in adult individuals. Eur J Endocrinol 165(5):723-728 


\section{EH P Adiponectin expression is correlated to physical activity in patients affected by cystic fibrosis}

\author{
R. Polito ${ }^{1}$, A. Elce ${ }^{2}$, E. Nigro ${ }^{3}$, M. L. Monaco ${ }^{4}$, \\ M. Gelzo ${ }^{5}$, P. Iacotucci ${ }^{6}$, F. Zarrilli, A. Daniele ${ }^{1}$
}

\begin{abstract}
${ }^{1}$ Dipartimento di Scienze e Tecnologie Ambientali, Biologiche, Farmaceutiche, Università della Campania "Luigi Vanvitelli", Caserta, Italy;

${ }^{2}$ Università Telematica Pegaso, Naples, Italy;

${ }^{3}$ Dipartimento di Scienze Cardio-Toraciche e Respiratorie, Università degli Studi della Campania, "Luigi Vanvitelli", Via Leonardo Bianchi clo Ospedale Mondaldi, 80131 Naples, Italy; ${ }^{4}$ CEINGE-Biotecnologie avanzate, Naples, Italy;

${ }^{5}$ Dipartimento di Medicina Molecolare e Biotecnologie Mediche, Università di Napoli Federico II, Naples, Italy;

${ }^{6}$ Centro Regionale Fibrosi Cistica Adulti, Dipartimento di Scienze Mediche Traslazionali, Università di Napoli Federico II, Naples, Italy;

${ }^{7}$ Dipartimento di Bioscienze e Territorio, Università del Molise, Isernia, Italy
\end{abstract}

Purpose: Adiponectin is an insulin-sensitizing, anti-atherogenic, antiinflammatory hormone circulating at different oligomerization state (HMW, MMW and LMW). The HMW represent the most biologically active oligomers. Cystic fibrosis (CF) is a respiratory genetic disease with a progressive decline of lung functions. Recently, we demonstrated that physical activity improves CF outcome. In this study, we characterized levels of total adiponectin and its HMW oligomers in adult CF patients that performed physical exercise.

Methods: $116 \mathrm{CF}$ patients and 98 ageand sex-matched controls were recruited. Patients were divided into two groups (58 versus 58 ) on the basis of supervised physical activity performed in the last three years. In the serum of patients and controls, total adiponectin and its HMW levels were measured by ELISA and western blot.

Results: Total adiponectin and its HMW levels are significantly more expressed in CF patients compared to health and matched population. Physical exercise significantly improves lipid and glycemic profile in CF patients. Furthermore, the levels of total adiponectin and its HMW oligomers decrease in $\mathrm{CF}$ patients that performed physical activity. Finally, total adiponectin resulted negatively correlated to lipid profile as well as to FEV\% decrease in CF patients that performed physical activity.

Conclusions: Our results demonstrated that a regular physical activity has metabolic and anthropometric advantages in CF patients. Among these, total Adiponectin serum concentrations and its HMW. Furthermore, the negative correlation of adiponectin to lipid profile in $\mathrm{CF}$ patients as well as to FEV \% decrease indicates that this adipokine may be considered an inflammatory biomarker in $\mathrm{CF}$ disease.

\section{EH P \\ $12 \mathrm{~min}$ walk/run test as a meter of cognitive fitness: a study on Italian students population}

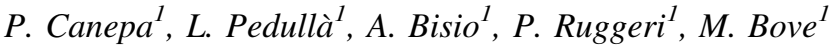 \\ ${ }^{1}$ Dipartimento di Medicina Sperimentale, Università degli Studi di \\ Genova, Genova, Italia
}

Purpose: In addition to common physical-health benefits, a good fitness condition has been associated with improvements in cognitive functions, in particular in processes involved in executive control such as selection, scheduling, perception, memory, and action [1]. Among them, working memory (WM), defined as the limited capacity storage system involved in the maintenance and manipulation of information over short time spans, is linked to several complex cognitive functions and has important implications in children's ability to acquire knowledge and new skills [2].

Despite the wide use in the Italian scholar system of field tests such as the $12 \mathrm{~min}$-walk/run test (12mWR) to evaluate individual maximal oxygen uptake [3], the students' population fitness condition was never associated to WM performance.

Therefore, the aim of this study was to investigate the correlation between the $12 \mathrm{mWR}$ and WM performance in young healthy subjects in school age.

Methods: Thirty participants (16 females, mean age $18 \pm 5$ years, range 10-24) performed two WM tasks, namely the Paced Auditory Serial Addition Test (PASAT) and the Symbol Digit Modalities Test (SDMT) and a walk/run test of $12 \mathrm{~min}$. Subjects were asked to cover the longest distance in $12 \mathrm{~min}$, and they were free to adjust their speed.

PASAT test was administered through an audio track reproduced by a personal computer. The audio track was composed by sixty stimuli applied at the distance of $3 \mathrm{~s}$ each other. In the SDMT participants had to pair specific numbers with given geometric figures in $90 \mathrm{~s}$. The results of two repetitions of PASAT and SDMT were averaged and then normalized on years of education.

Statistical analysis included Pearson's correlation between the $12 \mathrm{mWR}$, age and the normalized scores obtained at the WM tasks.

Results: Mean $12 \mathrm{mWR}$ performance was $2106 \pm 96 \mathrm{~m}$, whilst mean WM scores were $47.96 \pm 2.70$ for PASAT and $69.23 \pm 2.42$ for SDMT. We found significant correlations between the $12 \mathrm{mWR}$ and PASAT $(r=0.46, p=0.009)$ and between the $12 \mathrm{mWR}$ and SDMT $(r=0.43, p=0.017)$. No significant correlations were observed between age and the cognitive tests, nor between age and the $12 \mathrm{mWR}$.

Conclusions: The present findings suggested that the $12 \mathrm{mWR}$ is a good predictor of WM performance. The easy administration and the strong correlation with cognitive functions make the $12 \mathrm{mWR}$ a recommended indicator of academic achievement of students and a suggested tool to use in order to schedule personalized school activity. References

1. Kao SC, Westfall DR, Parks AC, Pontifex MB, Hillman $\mathrm{CH}$ (2017) Muscular and aerobic fitness, working memory, and academic achievement in children. Med Sci Sports Exerc 49(3):500-508

2. Baddeley A (2003) Working memory: looking back and looking forward. Nat Rev Neurosci 4(10):829

3. Mayorga-Vega D, Bocanegra-Parrilla R, Ornelas M, Viciana J (2016) Criterion-related validity of the distance-and time-based walk/run field tests for estimating cardiorespiratory fitness: a systematic review and meta-analysis. PLoS One 11(3):e0151671 


\section{EH P}

Effects of water-based aerobic training

with and without ginger supplement on double product and oxidative stress in obese women with breast cancer

\author{
S. Shokohyar ${ }^{1}$, N. Karimi ${ }^{2}$, V. Dabidi Roshan ${ }^{2}$, \\ M. Alkhatib ${ }^{3}$, E. Cè ${ }^{1}$, E. Limonta ${ }^{1}$, G. Coratella ${ }^{1}$, \\ S. Rampichini ${ }^{1}$, S. Longo ${ }^{1}$, F. Esposito ${ }^{1}$
}

${ }^{I}$ Department of Biomedical Sciences for Health, University of Milan, Milan, Italy;

${ }^{2}$ Department of Sport Physiology, Faculty of Physical Education and Sport Sciences, University of Mazandaran, Babolsar, Iran;

${ }^{3}$ Department of Experimental Medicine and Surgery, University of Rome "Tor Vergata", Rome, Italy

Purpose: In women with breast cancer (BC), obesity and overweight, the main cause of oxidative stress, are associated with cardiovascular risk. The use of non-pharmacologic strategies, such as water-based aerobic exercise and herbal supplementation could represent effective means to prevent diseases without any side effects caused by oxidative stress. Thus, the purpose of this study was to investigate the effect of a six-week, water-based exercise with and without ginger supplementation (Zingiber officinale) on double product (DP), oxidative stress in obese women with BC.

Methods: Forty women with breast cancer (age $48 \pm 5.4$ years; body mass $76 \pm 9 \mathrm{~kg}$; fat mass $41.8 \pm 4 \%$; body mass index $31.3 \pm 3.7 \mathrm{~kg} / \mathrm{m}^{2}$ ) have been voluntarily recruited to participate in study. Participants were randomly assigned to four groups of 10 people each: placebo, water-based exercise, ginger supplement and water-based exercise combined with ginger supplement. Subjects in the ginger and the combined groups received 4 capsules per day for 6 weeks, each capsule containing $750 \mathrm{mg}$ of ginger supplement. The progressive water-based exercise training program was performed in the range between $50 \%$ and $75 \%$ of heart rate reserve, 4 times a week for 6 weeks. The blood sampling was collected before and after the 6-week treatment to assess blood markers. To assess the glutathione peroxidase (GPx), malondialdehyde (MDA) and nitric oxide (NO) levels and other biochemical analyses, the ELISA method was used. The level of significance was set at $\alpha \leq 0.05$.

Results: Although the ginger supplementation and the water-based exercise resulted in a reduction in DP rest and MDA levels and an increase in GPx and NO compared to baseline, combined group showed significantly larger positive effect in all the outcomes, compared to the water-base exercise or ginger supplement alone.

Conclusions: Non-drug strategies, such as water-based exercise and ginger supplementation may play an important role in improving the quality of life in obese women diagnosed with BC by reducing cellular inflammation and stress.

\section{0b EHP \\ Physical activity in organ transplant}

\section{Garufi, A. Mallamace}

University of the Studies of Messina, Italy; Department of Biomedical Sciences, Dental, Morphological and Functional Images, CdLM

Science and Techniques of Preventive and Adapted Physical Activity

Purpose: Recent studies show that physical activity can counteract the side effects of immunosuppression: weight gain, cardiovascular and metabolic diseases. The goal of this study is to demonstrate that physical activity may be a key therapy for achieving the wellbeing of the patient transplanted.

Methods: The Center for sports medicine after a basic evaluation, will direct the patient to a gym certified on the territory where, a graduate in sports science trained, will follow the patient and will help you choose a physical activity with supervision (in the gym); Unsupervised (home); Recreational or competitive sports.

Training sessions should be minimum 3 for week, divided into $30 \mathrm{~min}$ of aerobic activity (intensity that matches your aerobic threshold) on a 30 min aerobics machine or free body and muscle strengthening with 2 series from 15-20 reps per muscle group.

Results: After 1 year you will notice the following changes: Increase in muscle strength; Increased bone density; Improving the functioning of the graft; Weight decreased; Decreased levels of cholesterol; Decrease the development of chronic diseases due to immunosuppressive therapy. Conclusions: Transplant centers, should direct their patients, already selected, to benefit from an exercise program prescribed by a specialist in sports medicine, with the aim of promoting physical activity to improve the health conditions and quality of life of organ transplant patients.

\section{References}

Macdonald JH, Kirkman D, Jibani M (2009) Kidney transplantation: a systematic review of international and observational studies of physical activity on intermediate outcomes. Adv Chronic Kidney Dis

European Directorate for the Quality of Medicines of healt Care (EDQM). European Council's Committee on Organ Transplantation (CD-P-TO) 


\section{SATURDAY ORAL SESSION II}

\section{MOVEMENT LEARNING AND PSYCHOPHYSIO- LOGICAL DEVELOPMENT}

\author{
111 ML K \\ Predictability, variability and individual styles \\ in throwing
}

\author{
A. d'Avella $^{1,2}$, A. Maselli $^{2}$, P. Tommasino ${ }^{2}$, B. Cesqui $^{3}$, \\ M. Russo ${ }^{3}$, F. Lacquaniti ${ }^{2,3}$ \\ ${ }^{1}$ Department of Biomedical and Dental Sciences \\ and Morphofunctional Imaging, University of Messina, Italy; \\ ${ }^{2}$ Laboratory of Neuromotor Physiology, Fondazione Santa Lucia, \\ Rome, Italy; \\ ${ }^{3}$ Centre of Space Bio-medicine and the Department of Systems \\ Medicine, University of Rome Tor Vergata, Italy
}

Purpose: Sensorimotor control is often investigated in laboratory settings using controlled experimental paradigms and simple tasks involving a few degrees-of-freedom. Whether the control strategies uncovered in simple tasks are also valid to solve complex sensorimotor problems such as those encountered in many sports is an open question. Prediction and coordination of many degrees-of-freedom in naturalistic and unconstrained conditions may allow for potentially multiple solutions and different individual strategies. We aimed at characterizing how the predictability and the variability of throwing actions are related to individual styles.

Methods: We recorded whole-body kinematics from twenty nonexpert participants performing unconstrained overarm throws at four different targets placed on a vertical plane. To characterize individual throwing styles and the spatiotemporal structure of the information embedded in the kinematics of the throwing action about the outgoing ball direction, we introduced a novel dimensionality reduction technique. To assess how variability in throwing affects performance we developed a novel method to identify different components of performance considering the individual distributions of ball release parameters.

Results: Throwing actions differed considerably across individuals and could be classified in a limited number of styles using a lowdimensional representation of whole-body kinematics. For most participants it was possible to predict the region where the ball hit the target plane, with an accuracy above $80 \%$, as early as $400-500 \mathrm{~ms}$ before ball release. The spatiotemporal structure of throw predictability differed across individuals and the body parts that provided the most informative cues about the action outcome varied with the throwing style and during the time course of the throwing action. Throwing performance also differed considerably across individuals and could be related to individual choices of mean ball release parameters, noise, and alignment of the release parameter covariance with the local curvature of the performance function.

Conclusions: Novel analytical methods to compactly represent and classify whole-body actions and to assess the impact on performance of action variability allow to characterize predictability, variability, and individual styles in throwing.

Reference

Maselli et al (2017) Where are you throwing the ball? I better watch your body, not just your arm! Front Hum Neurosci 11:505

\section{ML O \\ Motor unit conduction velocity at different joint angles}

\author{
M. Cogliati, A. Cudicio, C. Orizio, F. Negro
}

Department of Clinical and Experimental Sciences, University of Brescia, Italy

Purpose: The aim of this study was to evaluate the dependence of motor unit conduction velocity (MUCV) on the length of the muscle. Methods: Muscle length was modified considering three different ankle angles: $90^{\circ}, 110^{\circ}$ and $130^{\circ}$. For each angle the maximal voluntary contraction (MVC) was measured during static ankle dorsiflexion. High-density surface electromyography (HD-sEMG, 128 channels) was recorded from the tibialis anterior muscle (TA) in six young individuals. Subjects executed a volitional effort in which the output tension changed in a trapezoidal ramp fashion (15 s transient and $40 \mathrm{~s}$ steady contraction). Two levels of steady contraction were investigated: $10 \%$ and $20 \%$ of MVC. Using a novel decomposition technique based on HD-sEMG processing (Negro et al. 2016), the individual MUCV values were estimated during the trapezoid steady part. The decomposition was performed at each ankle angle independently, and the MU action potentials were not tracked across different muscle lengths.

Results: MVC mean values at $90^{\circ}$ and $130^{\circ}$ were $91.78 \%$ and $78.77 \%$ of $110^{\circ}$ value respectively. Considering that there was no statistical difference between the MUCV estimations calculated at 10 and $20 \%$ MVC, the values were grouped. The average CV was $4.00 \pm 0.54 \mathrm{~m} /$ $\mathrm{s}$ for $90^{\circ}, 3.88 \pm 0.19 \mathrm{~m} / \mathrm{s}$ for $110^{\circ}$ and $3.77 \pm 0.37 \mathrm{~m} / \mathrm{s}$ for $130^{\circ}$. One-Way Anova analysis showed a weak effect between the three conditions $(\mathrm{P}=0.04)$.

Conclusion: MUCV changes were weakly related to the different muscle lengths. Explanation of our results should consider that the muscle fiber can be approximated as a constant volume system and that, from the cable theory, the smaller the diameter the lower the CV is. On these bases, the reduction of muscle fibers transverse diameter during muscle elongation when the ankle angle increases from $90^{\circ}$ to $130^{\circ}$ could be a possible explanation for our results.

\section{Reference}

Negro F et al (2016) J Neural Eng 13(2):026027

\section{ML O}

The comprehension of grapho-motor skills, related to motor behavior, age and gender in school children

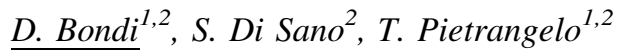 \\ ${ }^{I}$ Functional Evaluation Laboratory, University "G. d'Annunzio" \\ of Chieti-Pescara, Italy; \\ ${ }^{2}$ Department of Neuroscience, Imaging and Clinical Science, \\ University "G. d'Annunzio" of Chieti-Pescara, Italy
}

Purpose: This study was aimed to evaluate the grapho-motor skills in school children with a technological device, and to correlate them with motor control, functional performance and motor behavior, taking into account the gender variable, the anthropometric characteristics and the sport participation.

Methods: We used a tablet pc to assess the figure-tracing skills (number of strokes, pressure of pen, average speed and oscillations of lines drawn with respect to the reference line), among 80 schoolchildren of 2 nd ( 25 females and 23 boys) and 4th grade ( 15 females and 17 males). We also assessed the motor coordination (with KTK 
test), the static postural control (with a baropodometric-stabilometric platform), BMI (Body Mass Index) and handgrip strength, and asked for sport engagement.

Results: We found several differences in the class and gender comparisons. Handgrip strength influenced the strategies to accomplish the grapho-motor task. Sport participation influenced the outcomes. The different domains of subject's profile and motor behavior showed specific relations.

Conclusions: Findings showed that static postural control, body coordination, strength and grapho-motor skills are separately connoted and undergo different developmental models. Sport participation worth to be taken into account. Gender and age variables are strongly related to the motor behavior and demonstrate their effect in several ways, concerning different domains.

References

Flatters I, Hill LJB, Williams JHG, Barber SE, Mon-Williams M (2014) Manual control age and sex differences in 4 to 11 year old children. PLoS One $9(2)$

Scordella A, Di Sano S, Aureli T, Cerratti P, Verratti V, Fanò-Illic G, Pietrangelo $\mathrm{T}$ (2015) The role of general dynamic coordination in the handwriting skills of children. Front Psychol 6:580

Giammarco E, Di Sano S, Aureli T, Cerratti P, Fanò-Illic G, Pietrangelo T (2016) Psychological and physiological processes in figure-tracing abilities measured using a tablet computer: a study with 7 and 9 years old children. Front Psychol 7:1528

\section{ML O \\ Tool dependent motor imagery effects on primary motor cortex excitability in expert fencer}

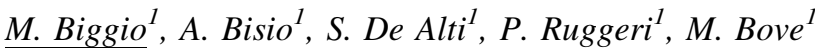

${ }^{1}$ Department of Experimental Medicine, Section of Human Physiology and Centro Polifunzionale di Scienze Motorie, University of Genoa, 16132, Genoa, Italy

Purpose: It is known that handling the sport-specific implement during motor imagery (MI) affects this mental process in athletes inducing a better isochrony between real and imagined movement and a facilitation in cortical excitability (Fourkas et al. 2008; Bisio et al. 2014; Wang et al. 2014). In the present work we tested whether, in a group of fencers, the èpèe strong predictor of cardiovascular disease and mortality [1] and often coexists with other cardiovascular risk factors, such as obesity. Previous studies on obese populations have shown improvements in parasympathetic reactivation following 3-6 months of exercise and diet interventions [2]. The aim of this study was to evaluate the effects of a 3-week exercise and nutritional inpatient intervention program on parasympathetic reactivation indices in severe obese adults.

Methods: 36 obese adults $(12 \mathrm{M}-24 \mathrm{~F}, \quad 46.3 \pm 12$ years, $41.44 \pm 5.33 \mathrm{~kg} / \mathrm{m}^{2}$ ), without major cardiovascular or neurologic disease, performed a maximal cardiopulmonary exercise test (CPET) on a cycle ergometer before and after the 3-week intervention, which included supervised aerobic and resistance exercise (80-120 min/day at low-moderate intensity, 5-6 days/week) combined with a nutritional caloric deficit of $800-1000 \mathrm{kcal} / \mathrm{day}$. Parasympathetic reactivation was assessed in the post-exercise period $(120 \mathrm{~s})$ through the analysis of short-term time constant of heart rate recovery (HRR) (T30 min), HRR indices (HRR60s and HRR120s) and the recovery of heart rate variability (HRV) indices (root mean square of successive differences of R-R intervals, RMSSD).

Results: After the intervention a significant decrease in T30 min (from $259.1 \pm 101.7$ to $220.6 \pm 78.2 \mathrm{~s}, \Delta \%=-14.88, \mathrm{p}=0.003$ ) with a concomitant significant increase in HRR60 s (from $29.1 \pm 9.5$ to $32.8 \pm 10.0 \mathrm{bpm}, \Delta \%=12.55 \% ; \mathrm{p}=0.004$ ) and HRR120 s (from $45.7 \pm 11.4$ to $53.0 \pm 13.0 \mathrm{bpm}, \Delta \%=16.10 \%, \mathrm{p}<0.001)$ were reported. Similarly, RMSSD was significantly higher at 60, 90 and $120 \mathrm{~s}$ after the program $(\mathrm{p}<0.05)$.

Conclusions: Improved HR and HRV recovery indices after maximal CPET denoted faster parasympathetic reactivation following the intervention. For the first time this study showed that improvements in ANS function can be obtained in severe obese patients in just 3 weeks of supervised exercise and nutritional intervention.

References

1. Qiu S et al (2017) JAHA 6(5)

2. Nagashima J et al (2010) Am J Cardiol 56(1):79-84

\section{ML O \\ Cognitive cost and brain activity during dual tasks: an fNRIS study in young adults}

L. Pedullà ${ }^{1}$, A. Bisio ${ }^{1}$, L. Bonzano ${ }^{2}$, P. Canepa ${ }^{1}$, S. Adascalitei ${ }^{1}$, G. Brichetto ${ }^{3}$, M. Bove ${ }^{I}$

${ }^{1}$ Dipartimento di Medicina Sperimentale, Università di Genova, Genova, Italia;

${ }^{2}$ Dipartimento di Neuroscienze, riabilitazione, oftalmologia, genetica e scienze materno-infantili, Università di Genova, Genova, Italia ;

${ }^{3}$ Area di Ricerca Scientifica, Associazione Italiana Sclerosi Multipla, Genova, Italia

Purpose: During sport competitions, athletes are engaged in simultaneous execution of motor and cognitive tasks (dual tasks, DT), with possible occurrence of cognitive-motor interference (CMI) [1], i.e., a reduction of the outcome quality during DT, which could compromise their performance.

Identifying sounds from the field and performing fast mental operations are cognitive demands frequently present in many sports, but their different impact on athletes' performance has never been compared. Moreover, the neural mechanisms underlying CMI are still unclear [2]. Cognitive DT cost (i.e., performance at [single task dual task]/single task $\times 100$ ) is an index that can help understanding the strategies adopted by athletes during the competition, and thus providing indications for better training programs.

Aim of the study was to investigate CMI and the neural substrates activity during DT in young adults.

Methods: Five subjects ( 3 males, mean age $=31 \pm 2$ years) were evaluated while performing two motor tasks, i.e., walking on straight and curvilinear paths, and two cognitive tasks, namely a discrimination task dealing with the identification of different sounds from the surrounding environment and a serial subtraction task. The combination of motor and cognitive tasks composed the DT paradigms and cognitive DT cost was calculated. During all single and DT conditions, brain activity was evaluated by means of a functional near infrared spectroscopy (fNIRS) system, an innovative portable tool allowing to detect cortical activation during movement.

Results: We observed negative cognitive DT cost (indicating better performance during DT compared to single task) during the sound identification task, either in straight and curvilinear paths. Conversely, serial subtraction performance decreased in DT conditions, as indicated by positive DT cost values.

Further, fNIRS contrasts revealed that the neural DT cost was associated with activity of sensorimotor and associative parietal areas for 
the serial subtraction task, and with the premotor area for the sounds identification task.

Conclusions: Attention to the sounds from the environment seems to improve during a concurrent motor task and showed a low neural DT cost. On the other hand, since computational demands impacted on participants' performance, training programs in order to improve this expertise during DT could be useful to reduce CMI and neural DT cost during open skill sports.

Acknowledgements: This study was supported by the Italian Multiple Sclerosis Foundation (2016/B/4)

References

1. Yogev-Seligmann G, Hausdorff JM, Giladi N (2012) Do we always prioritize balance when walking? Towards an integrated model of task prioritization. Mov Disord 27(6):765-770

2. Ruthruff E et al (2001) Processing bottlenecks in dual-task performance: structural limitation or strategic postponement? Psychon Bull Rev 8:73-80

\section{ML O}

\section{Topographical organization of human red nucleus as revealed by connectivity-based segmentation: implications in motor control}

\author{
A. Cacciola $^{1}$, G. A. Basile ${ }^{1}$, A. Favaloro ${ }^{1}$, S. Bertino ${ }^{1}$, \\ L. Magaudda ${ }^{1}$, G. Anastasi $^{1,}$ A. Duca ${ }^{2}$, D. Milardi $^{1,2}$, \\ G. C. Basile ${ }^{I}$, D. Di Mauro ${ }^{I}$, F. Trimarchi ${ }^{I}$ \\ ${ }^{1}$ Department of Biomedical, Dental Sciences and Morphological \\ and Functional Images, University of Messina, Italy; \\ ${ }^{2}$ IRCCS Centro Neurolesi “Bonino-Pulejo”, Messina, Italy
}

Purpose: To explore structural connectivity and topographical organization of the Red Nucleus (RN), key structure in the neural control of motor function. The Red Nucleus (RN) is a large nucleus located in the ventral midbrain. In monkeys, it is subdivided into a small caudoventral magnocellular part (mRN) and a large rostrodorsal parvicellular part (pRN).These distinct structural regions are part of functionally different networks, both important in motor learning and control: mRN receives projections from motor cortex, interposed nuclei and the spinocerebellum and regulates skilled movements of the distal upper limbs via the rubrospinal tract; $p R N$ is part of the dento-neorubro-olivary loop involved in motor learning and memory of motor parameters. In humans, less is known about its structural organization and hypotheses about its functions remain highly speculative yet, despite functional neuroimaging studies suggest its involvement in complex motor functions.

Methods: Herein, we use high-quality 3T structural and diffusion MRI data from the Human Connectome Project (HCP) and CSD tractography with a connectivity-based segmentation approach, in order to identify topographically distinguished subregions of the RN according to their different cortical and subcortical connectivity profiles.

Results: We tracked connections of $\mathrm{RN}$ both with inferior olivary nuclei (IONs), interposed nuclei (INs) and dentate nuclei (DNs), as well as with frontal motor and associative cortices.

We found that each RN can be subdivided according to its connectivity into two clusters: a large dorsolateral one, more connected with DNs and IONs, and a smaller ventromedial one, more connected with
IN. Topographical organisation of connections between cortical areas and these two clusters was also evaluated.

Conclusions: Our results are in line with previous literature and could represent a step toward a better understanding of how RN exerts its motor control functions in humans.

\section{References}

Stanton GB (1980) Topographical organization of ascending cerebellar projections from the dentate and interposed nuclei in Macaca mulatta: an anterograde degeneration study. J Comp Neurol 190(4):699-731. https://doi.org/10.1002/cne.901900406.

Larsen KD, Yumiya H (1980) The red nucleus of the monkey Topographic localization of somatosensory input and motor output. Exp Brain Res 40(4):393-404. https://doi.org/10.1007/bf00236148. Humphrey DR, Gold R, Reed DJ (1984) Sizes, laminar and topographic origins of cortical projections to the major divisions of the red nucleus in the monkey. J Comp Neurol 225(1):75-94. https://doi.org/10.1002/cne.902250109.

Kennedy PR, Gibson AR, Houk JC (1986) Functional and anatomic differentiation between parvicellular and magnocellular regions of red nucleus in the monkey. Brain Res 364(1):124-136. https://doi.org/10.1016/0006-8993(86)90993-5.

Kwon HG, Jang SH (2014) Differences in neural connectivity between the substantia nigra and ventral tegmental area in the human brain. Front Hum Neurosci 8:41. https://doi.org/10.3389/ fnhum.2014.00041.

Nioche C, Cabanis EA, Habas C (2009) Functional connectivity of the human red nucleus in the brain resting state at 3T. AJNR. 30(Februar):396-403. https://doi.org/10.3174/ajnr.a1375.

Milardi D, Cacciola A, Cutroneo G et al (2016) Red nucleus connectivity as revealed by constrained spherical deconvolution tractography. Neurosci Lett 626:68-73. https://doi.org/10.1016/ j.neulet.2016.05.009.

\section{TRAINING, PERFORMANCE AND EVA- LUATION METHODS 5}

\section{TP5 K}

Small Sided Games versus Match Play: comparison of external and internal training load in junior soccer players

\author{
M. Panascil ${ }^{1,2}$, A. Cittadino ${ }^{3}$, A. La Torre ${ }^{4}$, \\ D. Ferrari Bravo ${ }^{5}$, V. Ferrando ${ }^{1,2}$, E. Faelli ${ }^{1,2}$, \\ P. Ruggeri ${ }^{1,2}$
}

${ }^{1}$ Department of Experimental Medicine, Section of Human Physiology, University of Genoa, Genoa, Italy;

${ }^{2}$ Centro Polifunzionale di Scienze Motorie, University of Genoa, Genoa, Italy;

${ }^{3}$ F.C Catanzaro Calcio, Catanzaro, Italy;

${ }^{4}$ Department of Biomedical Sciences for Health, University of Milan, Italy;

${ }^{5}$ FC Juventus, Turin, Italy

Purpose: Small-Sided Games (SSGs) represent a widely used training modality to combine physiological, technical and tactical demands in soccer players [1]. The aim of the study was to compare external and internal training load in junior soccer players between 
two different SSGs (only ball possession, SSG-P; ball possession and shuttle run after the pass, SSG-S) and match play (MP).

Methods: Sixteen junior soccer players (age $18.5 \pm 1.5$ years; weight $73.1 \pm 5 \mathrm{~kg}$; height $175 \pm 2.5 \mathrm{~cm}$ ) were recruited from a professional team and they were monitored during 10 official matches of corresponding age categories. Participants performed two different 4vs4 SSGs (SSG-P and SSG-S) of the same duration $(4 \times 4$ min3 min rest), each format two times/week, in a randomized order, for 4 weeks. SSG training and MP data were collected during the inseason period, using GPS for external load and rate of perceived exertion (RPE) for internal load. External measures of load during the SSGs and MP were total distance (TD), the percentage of distance run at high speed (\%DHS; $>14.4 \mathrm{~km} \mathrm{~h}^{-1}$ ), the number of high intensity accelerations (HA; $\geq 2 \mathrm{~m} \mathrm{~s}^{-2}$ ) and decelerations (HD; $\geq-2$ $\mathrm{m} \mathrm{s}^{-2}$ ). With regards to the predicted metabolic parameters, average metabolic power (AMP) and, in addition, the distance covered at high metabolic power (DHMP; $>20 \mathrm{~W} \mathrm{~kg}^{-1}$ ) were calculated.

Results: AMP of SSG-P $\left(11.26 \pm 0.76 \mathrm{~W} \mathrm{~kg}^{-1}\right)$ and SSG-S $\left(11.58 \pm 0.77 \mathrm{~W} \mathrm{~kg}^{-1}\right)$ were not statistically different, whereas both AMP of SSGs trainings were significantly higher than that of MP $\left(9.07 \pm 0.81 \mathrm{~W} \mathrm{~kg}^{-1} ; \mathrm{p}<0.05\right)$. The \%DHS was significantly higher during MP $(14.97 \pm 2.3)$ compared to either SSG-P or SSG-S $(8.24 \pm 1.87$ and $9.43 \pm 2.54$, respectively; $\mathrm{p}<0.05)$. HA and HD were significantly higher during both SSGs than during MP $(\mathrm{p}<0.05)$. DHMP was not significantly different in all conditions. RPE was significantly higher during MP $(7.67 \pm 0.66)$ than during either SSG-P $(4.92 \pm 0.63 ; \mathrm{p}<0.01)$ or SSG-S $(7.04 \pm 0.45 ; \mathrm{p}<0.05)$.

Conclusions: External load of SSGs was significantly higher than that of MP, whereas internal load, estimated by RPE, was significantly lower in SSGs formats than MP condition. These findings suggest that both SSGs can represent an effective training for fitness coaches in junior soccer players, to improve fitness and technical/tactical skills. Reference

Gaudino P, Alberti G, Iaia FM (2014) Estimated metabolic and mechanical demands during different small-sided games in elite soccer players. Hum Mov Sci 36:123-133

\section{TP5 O}

\section{Oxidative stress following acute exercise in young elité rugby players}

L. Beratto $^{1,2}$, A. Finco $^{3}$, D. Gorni ${ }^{3}$, R. Coriani $^{2}$, F. Abate Daga ${ }^{2,4}$, M. Gollin ${ }^{2,5}$

${ }^{1}$ Department of Clinical and Biological Sciences, University of Turin, Italy;

${ }^{2}$ Adapted Training and Performance Laboratory, University School of Motor and Sport Sciences, University of Turin, Italy;

${ }^{3}$ Oxidative Research Department, Cor. Con. International srl, Parma, Italy;

${ }^{4}$ Department of Medical Sciences, University of Turin, Italy;

${ }^{5}$ Department of Clinical and Biological Sciences, University of Turin, Italy

Purpose: Physical exercise increases the antioxidant protection and reduces oxidative stress level. Intense aerobic and anaerobic exercise increases oxygen consumption and altered the antioxidant/pro-oxidant homeostasis. Reactive oxygen species (ROS) are produced during cellular oxygen metabolism. ROS are important in the mechanism adaptation and cell signalling, but elevated ROS generation may limit muscle strength and increase fatigue ${ }^{1}$. This study analyzes the effects of acute exercise on oxidative stress, antioxidant power and jump performance in young elite rugby athletes.

Methods: Eleven rugby players, 8 forwards and 3 backs (17 \pm 1 years, $181 \pm 4 \mathrm{~cm}, 92 \pm 14 \mathrm{~kg}$, BMI $28 \pm 4$, years of experience $7 \pm 3$ ) from the same team participated in this study. All their parents approved the attendance at this study and signed informed consent. Athletes performed jump test (Optogait, Microgate, Bolzano, Italy) squat jump (SJ) and countermovement jump (CMJ) before and after the training session. At the same time, saliva and blood samples were taken to evaluate reactive oxygen metabolites (dROMs), plasma (PAT) and saliva (SAT) antioxidant power by FRAS 5 system (H\&D srl, Parma, Italy). All players performed a medium intensity interval training $(16 \mathrm{~km} / \mathrm{h})^{2}$ between the two trials to develop aerobic and anaerobic energy system on different distances and rest (from 400 to $100 \mathrm{~m}$; from 60 to $30 \mathrm{~s}$ ).

Results: Both SJ and CMJ decreased significantly after the training session ( $\mathrm{SJ}-4 \%, \mathrm{p}=.005, \mathrm{CMJ}-5 \%, \mathrm{p}=.005)$. No difference was found in PAT $(\mathrm{p}=.26)$, SAT $(\mathrm{p}=.96)$ and d-ROMs test $(\mathrm{p}=.08)$. Moreover, a significant variation was detected in d-ROMs according to players position respectively in backs $(-8 \%, \mathrm{p}=.03)$ and forwards $(+10 \%, p=.008)$. No variation was observed in a jump performance in CMJ $(\mathrm{p}=.08)$ and $\mathrm{SJ}(\mathrm{p}=.11)$ in backs. Finally, we underline a significant variation in SJ $(-3 \%, \mathrm{p}=.05)$ and CMJ $(-6 \%, \mathrm{p}=.05)$ in forwards players.

Conclusion: Data shows a reduction in jump performance after the training session. Players position analysis underline that forwards show a greater increase of d-ROMs level to indicate a more fatigue to complete the training session. Even if the number of backs is low, the result highlights a better adaptation and higher capacity to manage the different interval distance proposed. Considering this, oxidative stress test can be a good way to evaluate and monitoring training effects on physical development.

\section{References}

1. Fatouros IG, Chatzinikolaou A, Douroudos II, Nikolaidis MG, Kyparos A, Margonis K, Michailidis Y, Vantarakis A, Taxildaris K, Katrabasas I, Mandalidis D, Kouretas D, Jamurtas AZ (2010) Time-course of changes in oxidative stress and antioxidant status responses following a soccer game. J Strength Cond Res 24(12):3278-3286

2. Coughlan GF, Green BS, Pook PT, Toolan E, O'Connor SP (2011) Physical game demands in elite rugby union: a global positioning system analysis and possible implications for rehabilitation. J Orthop Sports Phys Ther 41(8):600-605

\section{TP5 O}

The effects of 8 weeks of proprioceptive training on postural balance control and explosive strength in young track and field athletes

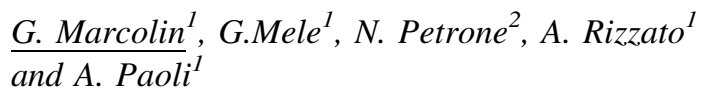 \\ ${ }^{1}$ Department of Biomedical Sciences, University of Padova, Italy; \\ ${ }^{2}$ Department of Industrial Engeneering, University of Padova, Italy
}

Purpose: Aim of the present study was to investigate the effects of 8 weeks of proprioceptive training on postural balance control and lower limb explosive strength in a group of young athletes.

Methods: 28 (14 male and 14 female) young track and field athletes (age $13.5 \pm 0.5$ years; weight $53.95 \pm 8.54 \mathrm{~kg}$; height 
$165.14 \pm 7.83 \mathrm{~cm}$ ) volunteered to participate in the study. They were randomly divided in two groups including the same number of male and female. The experimental group (EG) underwent the proprioceptive training while the control group (CG) underwent a traditional training including mobility, stretching and strength exercises without overloads. Each group trained twice a week. The postural balance evaluation consisted on a monopodalic standing test on a stabilometric platform and on a bipodalic test over an instrumented oscillating plate. The explosive strength was assessed by means of 2 tests: standing long jump (LJ) and counter movement jump (CMJ). Tests were performed before and after the 8 weeks of training.

Results: After the 8 weeks of training EG showed better scores in the postural balance control with respect to the CG both in static and dynamic tests. Jump tests showed no statistically significant differences both in EG and CG.

Conclusions: In young track and field athletes proprioceptive training allowed to increase postural balance control without affecting explosive strength. Therefore the introduction in training sessions of proprioceptive exercises could be useful to increase motor abilities and to reduce injury rate.

\section{References}

Heitkamp HC, Horstmann T, Mayer F, Weller J, Dickhuth HH (2001) Gain in strength and muscular balance after balance training. Int $\mathbf{J}$ Sports Med 22:285-290

Cressey EM, West CA, Tiberio DP, Kraemer WJ, Maresh CM (2007) The effects of ten weeks of lower-body unstable surface training on markers of athletic performance. J Strength and Cond Res 21(2):561-568

\section{TP5 O}

An exploratory systematic review of countermovement jump and squat jump operating procedures: reliability and usefulness of these measures/tests in a public health context

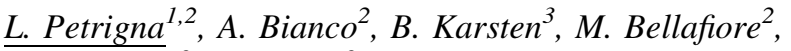 G. Battaglia ${ }^{2}$, A. Palma ${ }^{2}$}

${ }^{I}$ Sport and Exercise Sciences Research Unit, University of Palermo; ${ }^{2}$ Sport and Exercise Sciences Research Unit, SPPF Department, University of Palermo, Italy;

${ }^{3}$ Department of Exercise and Sport Science, Exercise and Sports, LUNEX International University of Health, Differdingen, Luxemburg

Purpose: countermovement jump (CMJ) and squat jump (SJ) are two vertical jumps (VJ) tests used to evaluate the lower limb reactive strength and the concentric power of the legs [1], respectively. Because the main problem of these two jumps is the lack of standardization in the test administration [2], the objectives were to gather information and investigate the standard aspects between CMJ and SJ protocols, to identify the most common method used or, if not evident, to design standardized operating execution procedure.

Methods: PubMed, Web of Science and Scopus databases were used for the electronic search (from January 2010 to June 2018). Original articles, systematic reviews and meta-analysis in which CMJ or SJ were used to assess muscular fitness in adolescences were eligible for further analysis. English language was imposed like limit. Abstracts, citations, statements and books were excluded. In a second moment, authors analysed the CMJ and SJ methods.

Results: A total of 104 studies (CMJ: 102, SJ: 46) met the inclusion criteria. The description of the CMJ and SJ test methodology was highly diverse within the literature with the protocol developed by
Bosco et al. [3], the one most used in the studies examined, that was utilized by five and three different authors, respectively.

Conclusions: Because there is no a standardized method for the two jumps, we created new CMJ and SJ protocols based on the positive aspects of the ones analysed.

\section{References}

1. Young W (1995) Laboratory strength assessment of athletes. New Stud Athl 10:89-96

2. Van Praagh E, Dore E (2002) Short-term muscle power during growth and maturation. Sports Med 32(11):701-728

3. Bosco C, Luhtanen P, Komi PV (1983) A simple method for measurement of mechanical power in jumping. Eur J Appl Physiol Occup Physiol 50(2):273-282

\section{PHYSICAL EXERCISE AS PREVENION AND THERAPY 4}

\section{EH4 K \\ Guided walking in hypertensive subjects: a selective therapy}

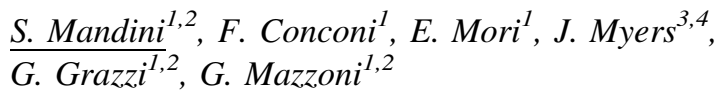

${ }^{1}$ Center of Biomedical Studies Applied to Sport, University

of Ferrara, Ferrara, Italy;

${ }^{2}$ Public Health Department, AUSL Ferrara, Ferrara, Italy;

${ }^{3}$ Veterans Affairs Palo Alto Health Care System, Palo Alto, CA, USA,

${ }^{4}$ Stanford University School of Medicine, Stanford, CA, USA

Purpose: To assess the effects of walking on the blood pressure of adult sedentary subjects with different degrees of hypertension.

Methods: 529 subjects with systolic blood pressure above $120 \mathrm{mmHg}$ were enrolled. Blood pressure, body weight, body mass index, waist circumference and walking speed were determined at enrolment and after 6 months. Walking sessions guided by exercise physiologists were organized.

Results: The average weekly walking time during the 6 months study period was up to $300 \mathrm{~min}$. Of the 296 subjects completing the project (182 women $59.6 \pm 9.0$ years, and 114 men, $65.4 \pm 8.6$ years) 27 had a baseline systolic blood pressure (SBP) $>160 \mathrm{mmHg}, 35$ between $150-159,70$ between $140-149,89$ between $130-139$ and 75 between $120-129 \mathrm{mmHg}$.

To analyze specifically the effects of walking on the blood pressure of the 296 hypertensive subjects finishing the project, they were subdivided into five subgroups with baseline SBP: $>160 \mathrm{mmHg}$, between 150-159, between 140-149, between 130-139 and between $120-129 \mathrm{mmHg}$. Highly significant reductions in SBP were documented in each subgroup with lowering of $-21.3 \mathrm{mmHg}$ in the subjects with baseline values $>160$, of -11.8 in the group 150-159, of -7.5 in the group 140-149, of -5.3 in the group 130-139 and -2.6 in the group $120-129 \mathrm{mmHg}$. Highly significant reductions were also documented for diastolic pressure, body weight, body mass index and waist circumference. These reductions were nearly identical within the various groups. Walking speed increased significantly and uniformly in all groups.

The study shows that in hypertensive sedentary subjects, walking is followed by marked reductions of SBP. Its lowering is proportional to baseline values: the higher the baseline values, the greater the blood pressure reductions observed. Walking safely and effectively 
lowers the blood pressure of hypertensive subjects and should be included as standard adjunctive therapy for hypertension.

\section{References}

Pescatello LS, MacDonald HV, Lamberti L, Johnson BT (2015) Exercise for hypertension: a prescription update integrating existing recommendations with emerging research. Curr Hypertens Rep 17(11):87

Ettehad D, Emdin CA, Kiran A et al (2016) Blood pressure lowering for prevention of cardiovascular disease and death: a systematic review and meta-analysis. Lancet 387(10022):957-967

Redon J, Mourad JJ, Schmieder RE, Volpe M, Weiss TW (2016) Why in 2016 are patients with hypertension not $100 \%$ controlled? A call to action. J Hypertens 34(8):1480-1488

Whelton PK, Carey RM, Aronow WS et al (2017) Guideline for the prevention, detection, evaluation, and management of high blood pressure in adults: executive summary: a report of the American College of Cardiology/American Heart Association Task Force on Clinical Practice Guidelines. Hypertension

Keywords: Hypertension, walking, exercise physiology

\section{EH4 O}

A 3-week exercise and nutritional intervention program improves post exercise cardiac autonomic recovery in obese adults

\author{
F. Gilli ${ }^{1}$, A. Callovini ${ }^{2}$, A. Fornasiero ${ }^{2}$, S. Skafidas ${ }^{1}$,

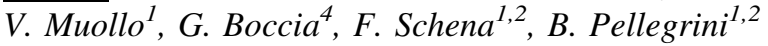 \\ ${ }^{1}$ CeRiSM, Sport Mountain and Health Research Centre, University \\ of Verona, Rovereto; \\ ${ }^{2}$ Department of Neurosciences, Biomedicine, and Movement Sciences, \\ University of Verona; \\ ${ }^{4}$ Department of Medical Sciences, University of Turin
}

Purpose: Impaired post-exercise parasympathetic reactivation, representing autonomic nervous system (ANS) dysfunction, is a strong predictor of cardiovascular disease and mortality [1] and often coexists with other cardiovascular risk factors, such as obesity. Previous studies on obese populations have shown improvements in parasympathetic reactivation following 3-6 months of exercise and diet interventions [2]. The aim of this study was to evaluate the effects of a 3-week exercise and nutritional inpatient intervention program on parasympathetic reactivation indices in severe obese adults.

Methods: 36 obese adults $(12 \mathrm{M}-24 \mathrm{~F}, \quad 46.3 \pm 12$ years, $41.44 \pm 5.33 \mathrm{~kg} / \mathrm{m}^{2}$ ), without major cardiovascular or neurologic disease, performed a maximal cardiopulmonary exercise test (CPET) on a cycle ergometer before and after the 3-week intervention, which included supervised aerobic and resistance exercise $(80-120 \mathrm{~min} /$ day at low-moderate intensity, 5-6 days/week) combined with a nutritional caloric deficit of $800-1000 \mathrm{kcal} / \mathrm{day}$. Parasympathetic reactivation was assessed in the post-exercise period $(120 \mathrm{~s})$ through the analysis of short-term time constant of heart rate recovery (HRR) (T30 min), HRR indices (HRR60s and HRR120s) and the recovery of heart rate variability (HRV) indices (root mean square of successive differences of R-R intervals, RMSSD).

Results: After the intervention a significant decrease in T30 min (from $259.1 \pm 101.7$ to $220.6 \pm 78.2 \mathrm{~s}, \Delta \%=-14.88, \mathrm{p}=0.003$ ) with a concomitant significant increase in HRR60 s (from $29.1 \pm 9.5$ to $32.8 \pm 10.0 \mathrm{bpm}, \Delta \%=12.55 \% ; \mathrm{p}=0.004$ ) and HRR120 s (from $45.7 \pm 11.4$ to $53.0 \pm 13.0 \mathrm{bpm}, \Delta \%=16.10 \%, \mathrm{p}<0.001)$ were reported. Similarly, RMSSD was significantly higher at 60,90 and $120 \mathrm{~s}$ after the program $(\mathrm{p}<0.05)$

Conclusions: Improved HR and HRV recovery indices after maximal CPET denoted faster parasympathetic reactivation following the intervention. For the first time this study showed that improvements in ANS function can be obtained in severe obese patients in just 3 weeks of supervised exercise and nutritional intervention.

References

1. Qiu S et al (2017) JAHA 6(5)

2. Nagashima J et al (2010) Am J Cardiol 56(1):79-84

\section{EH4 O}

The role for strain-echocardiography in discriminating between hypertrophic cardiomyopathy and athlete's heart in young athletes

\author{
A. Bonaiuto ${ }^{l}$, C. Tarozzo ${ }^{l}$, R. Restuccia ${ }^{l}, F$ Trimarchi $^{1,2}$, \\ D. Bruschetta ${ }^{1}$, L. Magaudda ${ }^{1,2}$, C. de Gregorio ${ }^{2,3}$ \\ ${ }^{I}$ Department of Biomedical, Dental Sciences and Morphological and \\ Functional Images, Postgraduate School of Sports Medicine. \\ University of Messina, Messina, Italy. \\ ${ }^{2}$ Sports Medicine Unit, University Hospital "G. Martino", Messina, \\ Italy. \\ ${ }^{3}$ Department of Clinical and Experimental Medicine, Cardiology \\ Unit, University Hospital of Messina, Messina, Italy
}

Purpose: Strain-echocardiography has been demonstrated to be a valid diagnostic tool for differentiating between pathological and physiological left ventricular hypertrophy $(\mathrm{LVH})$, both in the general population and in athletes. Impaired longitudinal deformation is considered as a potential marker of abnormal LV remodelling. However, only scanty data are available about strain-echocardiography findings in young athletes likely to have a borderline (grey-area) $\mathrm{LVH}$. We sought to assess strain function in a small group of young athletes with either athlete's heart or grey-area LVH.

Methods: Based on the pre-participation screening programme for competitive sports, we examined a population of young trained athletes (range 16-18 years) by ECG and Doppler-echocardiography. Based on both clinical and instrumental findings, we identified a small group likely to have non-physiological LVH (group A). Conventional echo-derived measurements and strain values were compared to those from an ageand body mass index-matched group of healthy athletes (group B) and a control group (group C). Left ventricular radial, circumferential and longitudinal deformation was recognized by vector-velocity strain analysis. 
Results: Four athletes (mean aged $16 \pm 1$ years) were found with ECG and echo signs of grey-area LVH. Echo-derived findings were compared to 5 healthy athletes $(17 \pm 0.9$ years $)$ and 5 controls (17 \pm 1.1 years). Higher LV mass and LV mass index were found in athletes from group $\mathrm{A}$ than in groups $\mathrm{B}$ and $\mathrm{C}$, but there were no significant discrepancies in functional indices (LV ejection fraction and fractional shortening). However, athletes in group A showed poorer longitudinal $(-18.8 \pm 2.6 \%)$, but better circumferential $(-33.6 \pm 6.5 \%)$ and radial $(39.1 \pm 0.6 \%)$ strain values than those in groups B and C.

Conclusions: Though numerically limited, this study confirms the presence of subclinical impairment in LV longitudinal deformation even in young athletes likely to have non-physiological LVH. Their preserved LV ejection fraction can be warranted by a greater radial and circumferential function.

\section{EH4 O}

\section{Mindful movement: exploring the association between interlimb coordination and cognitive functioning}

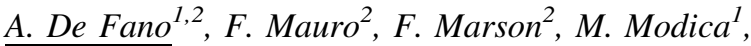 \\ C. Pesce ${ }^{1}$, J. Glicksohn ${ }^{3,4}$, T.D. Ben-Soussan ${ }^{* 2,3}$
}

\author{
${ }^{1}$ Department of Movement, Human and Health Science, Italian \\ University Sport and Movement Foro Italico, Rome, Italy; \\ ${ }^{2}$ Cognitive Neurophysiology Laboratory, Research Institute \\ for Neuroscience, Education, and Didactics, Patrizio Paoletti \\ Foundation, Assisi, Italy; \\ ${ }^{3}$ The Leslie and Susan Gonda Multidisciplinary Brain Research \\ Center, Bar-Illan University, Israel; \\ ${ }^{4}$ Department of Criminology, Bar-Ilan University, Israel
}

Purpose: Mindful Movement (MM) is a new category of exercise characterized by a high level of attention and awareness focused on body movements. MM showed beneficial effect on motor and cognitive functioning, but further studies are needed to understand what functions benefit most from these practices. Therefore, the aims of the current study were to investigate whether (1) the extensive practice of $\mathrm{MM}$ is positively associated with interlimb coordination, time production (TP), and divergent thinking (DT), and (2) there is a relationship between coordination and TP and DT.

Methods: Thirty-four healthy adults volunteered, including 11 Aikido (AIK) and 9 advanced Quadrato Motor Training (aQMT) practitioners, and 14 physically non-active controls (CTR). The participants performed the Time Production and Alternative Uses tasks followed by the homolateral interlimb coordination task.

Results: Practitioners of aQMT had significantly better coordinative performance and longer TP as compared to the AIK and CTR groups. However, no group differences in terms of DT emerged. Moreover, increased coordination efficiency was associated with longer TP. Furthermore, significant correlation between left in-phase interlimb coordination and DT was found only for aQMT.

Conclusions: $\mathrm{MM}$ is a potential way to improve coordination and cognitive functioning among adults, and can have potential benefits for populations suffering from cognitive and coordination deficits. References

Ben-Soussan TD, Glicksohn J, Berkovich-Ohana A (2015) From cerebellar activation and connectivity to cognition: a review of the Quadrato Motor Training. BioMed Res Int

Larkey L, Jahnke R, Etnier J, Gonzalez J (2009) Meditative movement as a category of exercise: implications for research. J Phys Act Health 6:230-238

\section{SPORT BIOMECHANICS AND TECHNOLOGY 1}

\section{SB1 K \\ In Vivo Kinematic Analysis PreAnd PostAcl Reconstruction During Single-Leg-Squat Using Dynamic RSA}

\author{
L. Bragonzoni $^{1,2}$, M. Bontempi ${ }^{1,2}$, \\ U. Cardinale $^{1}$, G. M. Marcheggiani Muccioli ${ }^{2}$, \\ D. Alesi ${ }^{2}$, S. Zaffagnini ${ }^{2}$ \\ ${ }^{1}$ Università di Bologna, Bologna (BO), Italia; \\ ${ }^{2}$ Clinica 2, Istituto Ortopedico Rizzoli, Bologna (BO), Italy
}

Purpose: The objective of this work was to in vivo analyze for the first time by means of Dynamic RSA the differences in kinematics between 2 different techniques for anterior cruciate ligament (ACL) reconstruction: the Non-Anatomical Double-Bundle (NADB) and the Over-The-Top with Lateral Plasty (SBLP) [1].

Methods: 27 patients with an isolated ACL injury were recruited. The average age was 26 years old. The patients were randomized in 2 different groups: 13 were reconstructed using the SBLP and 14 using the NADB technique. A Dynamic RSA device was used to evaluate patients the day before surgery and 18 months post-operatively. The patients were asked to perform a single leg squat using the injured leg: from standing to maximal allowed flexion (forward phase) and from maximal allowed flexion to standing (return phase). The motion parameters were evaluated using the Eulero. Data were statistically evaluated using the 1 -way ANOVA $(\mathrm{p}<0.05)$, to find significant differences during the movements.

Results: The maximal flexion allowed during the single leg squat was the same before and after ACL reconstruction (mean angle 67 degrees). The significant differences between the 2 groups regarded internal/external (IE) rotations at 18 month follow-up. The pre-operative IE pattern of NADB and SBLP groups did not shown significant differences: in the forward phase the IE rotations values ranged from -2 (external rotation) to +6 (internal rotation) ending with $+4^{\circ}$ at the full flexion; in the return phase the values ranged from $+4^{\circ}$ to $+8^{\circ}$. This pattern is expression of general instability of the knee. On the contrary, the 2 groups showed significantly different patterns after ACL reconstruction. The NADB group showed differences between the forward and the return phase. The forward phase had the following IE rotations values: $+2^{\circ}, 0^{\circ},+4^{\circ}$. The return phase: $+4^{\circ},+8^{\circ},+5^{\circ}$.

\section{References}

1. Zaffagnini S, Signorelli C, Bonanzinga T, Roberti Di Sarsina T, Grassi A, Budeyri A, Marcheggiani Muccioli GM, Raggi F, Bragonzoni L, Lopomo N, Marcacci M (2016) Technical variables of ACL surgical reconstruction: effect on post-operative static laxity and clinical implication. KSSTA 24(11):3496-3506

2. Clary CW, Fitzpatrick CK, Maletsky LP, Rullkoetter PJ (2013) The influence of total knee arthroplasty geometry on mid-flexion stability: an experimental and finite element study. J Biomech 46(7):1351-1357

Keywords: ACL, Dynamic RSA, Kinematic 


\section{SB1 O}

An innovative comprehensive multifactorial evaluation of 3D full skeleton posture and spine functional mobility for the quantitative morpho-functional characterization for APA in the elderly

\author{
M. D'Amico $^{1,2}$, E. Kinel $^{2,3}$, P. Roncoletta ${ }^{2}$, D. Bondi ${ }^{1}$, \\ T. Pietrangelo ${ }^{1}$
}

${ }^{I}$ Functional Evaluation Laboratory, Department of Neuroscience, Imaging and Clinical Science, University "G. d'Annunzio" of ChietiPescara, Italy;

${ }^{2}$ SMART Lab (Skeleton Movement Analysis and Advanced Rehabilitation Technologies) Bioengineering and Biomedicine

Company Srl, Pescara, Italy;

${ }^{3}$ Department of Rheumatology and Rehabilitation, Clinic

of Rehabilitation, University of Medical Sciences, Poznan, Poland

Purpose: During the last years our group built up a general framework to accurately quantitatively evaluate the postural/functional status of senior subjects to provide individualised training plans of adapted physical activities (APA) to contrast sedentary lifestyle, sarcopenia and low-grade chronic inflammatory status (Inflammaging). To achieve the "proper individualised APA" and to monitor its effect on the elderly quantitative measurements and functional tests are included. This paper aims to present the innovative developed protocol for Comprehensive Multifactorial Biomechanical Analysis (CMBA) of 3D full skeleton posture, spine morphology and functional mobility in the elderly.

Methods: A 3D stereophotogrammetric measurement of 27 body landmarks labelled by passive markers have been chosen to measure the 3D parametric biomechanical skeleton model 3D spine included [1]. For a good global functional status is of major importance to evaluate how efficient is the subject's posture and spine morphology and associated functional limitations. This requires integrating morphological characteristics with the information deriving from other measurements devices. Two channels SEMG are recorded following the SENIAM [2] recommendations to measure Multifidus bilateral activities to study Flexion Relaxation Phenomenon (FRP) [3]. A baropodometric platform measures pressure maps and vertical forces on each foot.

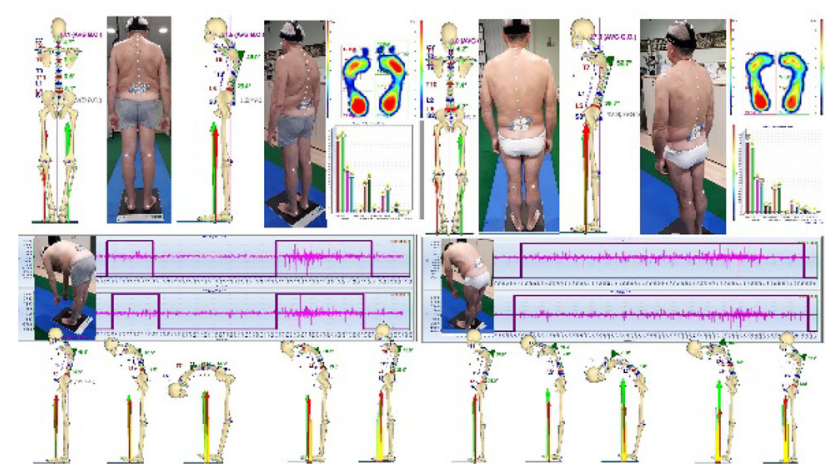

Results: Figure 1 shows the comparison of two elderly subjects. Differences in the 3D full skeleton posture are accounted for quantitative parameters identifying the 3D spine morphology, global and trunk balancing, associated left and right underfoot loads distributions, pressure maps (upper panels). The forward bendings highlight the differences in functional flexibility of the trunk considering the ROM of pelvis tilt and Lumbar and Thoracic spine flexions.
Multifidus SEMG shows normal FRP in the first subject (left lower panels) and abnormal FRP in the second subject (right lower panels) Conclusions: The innovative developed protocol for CMBA proved to be effective in unveiling the complexity of Biomechanics of human Posture and motor control mechanisms, confirming its accuracy and specificity for the planning and monitoring of "proper individualised APA" in the elderly. The data collection from different measurement devices integrated into a unique multi-factorial framework, provide a real added value in the comprehension of clinical and biomechanical phenomena.

\section{References}

1. D'Amico M, Kinel E, Roncoletta P (2017) Normative 3D optoelectronic stereo-photogrammetric posture and spine morphology data in young healthy adult population. PLoS One 12:e0179619. https://doi.org/10.1371/journal.pone.0179619

2. Hermens HJ, of the European Communities. Biomedical C, Programme HR (1999) European recommendations for surface ElectroMyoGraphy: results of the SENIAM project [Internet]. Roessingh Research and Development. http://books.google.es/ books?id=w7HgOwAACAAJ

3. D'Amico M, D'Amico G, Frascarello M, Paniccia M, Roncoletta P, Vallasciani M (2008) A 3-D skeleton model \& SEMG approach for integrated neck and low back pain analysis test batteries. Res Spinal Deform 140:79-84

\section{SB1 O}

Running with skis, biomechanical analysis of a recent evolution in cross country skiing technique

\author{
B. Pellegrini, C. Zoppirolli, F. Stella, L. Bortolan, \\ F. Schena
}

CeRiSM Research Centre "Sport, Mountain, and Health", Rovereto, Italy; Department of Neuroscience, Biomedicine and Movement, University of Verona, Verona, Italy

Purpose: The introduction of mass-start and sprint events in cross country skiing requires the skiers to attain high speed and/or rapid accelerations during a race. Recently, many skiers adapted the traditional diagonal stride technique (DStrad) by introducing on steep uphills a kind of "running" diagonal stride (DSrun), where gliding phase is minimal or absent. Here we compared the biomechanics of DStrad and DSrun, to evaluate the possible advantages of the new technique.

Methods: Eight elite skiers skied with roller skis on a treadmill at $10 \mathrm{~km} / \mathrm{h}$ and $10^{\circ}$ of slope, by using DStrad and DSrun. 3D kinematics of body segments, poling forces and plantar pressures were acquired simultaneously. Skiing cycle was divided in three phases, gliding, push off and leg swing. Joint angles were calculated on sagittal plane, and mechanical work was estimated from COM motion, work to climb against gravity and to overcome rolling friction.

Results: Cycle time (CT) was found to be significantly shorter for DSrun $(0.76 \pm 0.03 \mathrm{~s}$ vs. $1.06 \pm 0.07 \mathrm{~s})$; leg propulsion $(\sim 0.2 \mathrm{~s})$ and leg swing time $(\sim 0.45 \mathrm{~s})$ were similar and gliding phase much shorter for DSrun, $(0.10 \pm 0.04 \mathrm{~s}$ vs. $0.40 \pm 0.03 \mathrm{~s})$ than for DStrad accounting for the whole difference in CT.

At ski-ground contact, knee is at its maximal flexion for DStrad and it extended during gliding; on the contrary, knee is extended for DSrun at ground contact and it flexed just a bit before propulsion phase. Knee has different pattern of angular motion also during propulsion phase and peak force was reached earlier for DSrun. During swing, 
knee is much more flexed for DSrun $\left(\sim 115^{\circ}\right.$ vs. $\left.\sim 98^{\circ}\right)$. Mechanical work was $\sim 10 \%$ higher for DSrun than for DStrad.

Conclusions: The new technique allows to ski at higher movement frequency, thus augmenting propulsive contribution. Despite a higher mechanical work required, this technical evolution may allow to reach higher peak power and speed over short distances. Differences in joint kinematic and plantar force underlines that it is not just matter to avoid gliding phase and specific technical training may be required to effectively perform this new technique.

\section{SUNDAY ORAL SESSION I}

\section{BIOMOLECULAR AND NUTRITIONAL ASPECTS OF EXERCISE AND SPORT 1}

\section{SB1 O \\ Race walking body center of mass trajectory can be computed via inverse dynamics with a Kernel-based approach}

\author{
G. Pavei $^{1}$, G. Santin ${ }^{2}$ \\ ${ }^{1}$ Department of Pathophysiology and Transplantation, University \\ of Milan, Milano, Italy; \\ ${ }^{2}$ Institute of Applied Analysis and Numerical Simulation, University \\ of Stuttgart, Stuttgart, Germany
}

Purpose: Race walking gait is characteristic for many aspects (progression speed, pelvic movement, etc.) and from a mechanical point of view it is the only human gait that cannot be described via forward (FD) and inverse (ID) dynamics interchangeably (Pavei et al. 2017). The body center of mass (BCoM) computation and $\mathrm{BCoM}$ trajectory are the starting points for the mechanical analysis of locomotion. $\mathrm{BCoM}$ can be obtained by double integration from the ground reaction force signal (FD) or as weighted mean of the segments' center of mass (ID), ID is based on the assumption that body segments are rigid. Since all previous attempts to calculate ID BCoM based on different anthropometric tables failed (Pavei et al. 2017) the aim of this study was to develop a mathematical approach that could compute BCoM from kinematic data with a good approximation to FD as for other human gaits.

Methods: Race walking strides at increasing speed $(2.22-4.17 \mathrm{~m} / \mathrm{s}$, with $0.27 \mathrm{~m} / \mathrm{s}$ increment) were collected synchronously by a 8 -camera Vicon system (at $300 \mathrm{~Hz}$ ) and a Mercury LT med treadmill equipped with four 3D strain-gauge force traducers sampling at $900 \mathrm{~Hz}$. Subject was equipped with 18 markers and each acquisition lasted $1 \mathrm{~min}$. BCoM trajectory was computed from ID and FD and closed in a loop centred on $(0,0,0)$; difference between trajectories was calculated point-by-point with RMSE. Kernel-based regression was applied to the observed data to train a surrogate model that maps the kinematic data to the measured FD BCoM. Such model is then used to predict, with suitable accuracy, the FD BCoM of previously unseen kinematic data (Haasdonk and Santin 2018).

Results: ID BCoM trajectory shows a different shape and a mean RMSE of 0.008 m compared with FD. The Kernel surrogate resembles the same FD BCoM trajectory with a mean RMSE of $0.003 \mathrm{~m}$. Conclusion: The Kernel method is free from any anthropometric tables and rigid segment assumption and reaches satisfactory FD
BCoM trajectories, better than ID. Moreover, RMSE values are even smaller than ID vs. FD in running.

\section{References}

Pavei et al (2017) On the estimation accuracy of the 3D body center of mass trajectory during human locomotion: inverse vs. forward dynamics. Front Physiol 8:129

Haasdonk and Santin (2018) Greedy kernel approximation for sparse surrogate modeling, reduced-order modeling (ROM) for simulation and optimization: powerful algorithms as key enablers for scientific computing. Springer, pp 21-45

\section{BN1 K}

Skeletal muscle capillary density predicts muscle morphological adaptation to resistance exercise in older adults

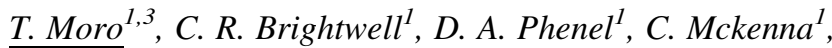

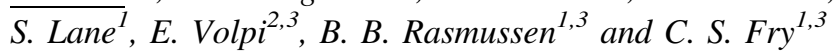 \\ ${ }^{1}$ Department of Nutrition and Metabolism, School of Health \\ Professions, University of Texas Medical Branch, Galveston, TX, \\ USA; \\ ${ }^{2}$ Department of Internal Medicine/Geriatrics, University of Texas \\ Medical Branch, Galveston, TX, USA; \\ ${ }^{3}$ Sealy Center on Aging, University of Texas Medical Branch, \\ Galveston, TX, USA
}

Introduction: Aging induces a substantial decrease in muscle capillarization, reducing the transport of nutrients, oxygen and hormones into muscle fibers. It is known that physical activity can improve muscle perfusion by increasing capillary density in young adults, however, its role in older adults is still controversial.

The aim of this study was to investigate the association between muscle capillary density and indices of muscle hypertrophy before and after 12 weeks of progressive resistance exercise training (RET). Methods: 19 subjects $(71.1 \pm 4.3$ years; $27.6 \pm 3.2$ BMI $)$ were studied before and after 12 weeks of RET. Pre and post-training measurements of muscle protein synthesis, muscle mass and strength were obtained. Percutaneous biopsies from the vastus lateralis muscle during a metabolic infusion study were taken to assess the change in muscle fiber characteristics and protein synthesis. Immunohistochemical analysis was used to quantify capillary density, myosin heavy chain (MyHC) isoform expression and cross-sectional area (CSA). Basal mixed muscle and myofibrillar protein fractional synthetic rate was assessed by stable isotope methodology.

Results: Basal muscle capillarization was highly correlated with lean body mass, sarcopenic index (SMI) and daily physical activity level $(\mathrm{p}<0.05)$. Muscle capillary density was also positively associated $(\mathrm{p}<0.05)$ with fiber type I frequency and fiber size (CSA). Moreover, higher basal capillarization was positively correlated $(\mathrm{p}<0.05)$ with a higher improvement in muscle fiber CSA and protein synthesis. RET increased muscle capillary density $(\mathrm{p}<0.05)$, which was also correlated with an increase in fiber CSA and MyHC type II fiber frequency.

Conclusion: Muscle fiber capillarization at baseline may be a predictive factor for improving muscle hypertrophy in response to RET in older adults. Increases in muscle fiber perfusion following RET are correlated with muscle growth and seems to be critical to achieve some muscle morphological adaptation 


\section{BN1 O}

\section{Effects of adiponectin on the human LHCN-M2 myoblasts differentiation}

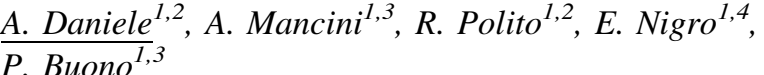

${ }^{1}$ CEINGE-Biotecnologie Avanzate S.c.a r.l., Naples, Italy; ${ }^{2}$ Dipartimento di Scienze e Tecnologie Ambientali Biologiche Farmaceutiche, Università degli Studi della Campania, "Luigi Vanvitelli", Caserta, Italy;

${ }^{3}$ Dipartimento di Scienze Motorie e del Benessere, Università "Parthenope" Naples, Italy;

${ }^{4}$ Dipartimento di Scienze Cardio-Toraciche e Respiratorie, Università degli Studi della Campania, "Luigi Vanvitelli", Via Leonardo Bianchi clo Ospedale Monaldi, Naples, Italy

Purpose: Myogenic differentiation is a complex event that involves different molecular modifications. Several factors, such as hormones and growth factors, can modulate myogenic proliferation and differentiation. Adiponectin, by its three widely expressed receptors AdipoR1, AdipoR2 and T-cadherin, exerts its metabolic effects mainly in liver and skeletal muscle increasing fatty acid oxidation, glucose uptake and lactate production [1]. To date, the role of Adiponectin on differentiation was not completely defined. In this scenario, we investigated the effects of adiponectin on human myogenic LHCN-M2 cell line [2], a novel species-specific system that has various applications in skeletal muscle research.

Methods: The human LHCN-M2 myoblast cells were cultured in growth medium (GM-15\% FBS) or in Differentiation Medium (DM) with $0.5 \%$ FBS, apotransferrin and insulin or with adiponectin $(0.1 \mu \mathrm{g} / \mathrm{ml})$ or with IGF-1 $(15 \mathrm{ng} / \mathrm{ml})$ or with adiponectin $(0.1 \mu \mathrm{g} / \mathrm{ml})$ and IGF-1 $(15 \mathrm{ng} / \mathrm{ml})$ for 4 days. Myogenic differentiation was assessed by Fusion Index (FI) and Myogenin and MyHC- $\beta$ mRNAs expression levels evaluation. The expression levels of AdipoRs and $\mathrm{T}$-cadherin were also determined in treated cells by RT $q \mathrm{PCR}$ and Western blot.

Results: We first demonstrated the expression of AdipoRs and T-cadherin on LHCN-M2 cells and successively investigated the effects of adiponectin on the LHCN-M2 cells differentiation. Furthermore, we examined the effects of adiponectin on LHCN-M2 cells exposed to IGF-1, a key factor regulating skeletal muscle development and growth. Interestingly, our preliminary results showed that adiponectin, in doseand time-dependent manner, reduced the proliferation of LHCN-M2 cells. In addition, we observed that adiponectin treatment induced myotube formation and significantly increased the early (Myogenin) and late (MyHC- $\beta$ expression) differentiation markers versus cells cultured with GM serum. The combined treatment with adiponectin and IGF-1 seems to have synergic effects on LHCN-M2 cells.

Conclusions: understanding the role and the potential functional connection between Adiponectin and IGF-1 may be of physiological and clinical relevance.

Acknowledgements: This work was supported by University of Naples Parthenope, "Bando per la ricerca individuale, annualità 2016" and "Bando per la Ricerca competitiva, triennio 2016-2018 quota C"

References

1. Orrù $S$ et al (2017) Int $J$ Mol Sci 14:18

2. Vitucci et al (2018) J Sports Sci 36:1630-1639

\section{BN1 O}

Hormone receptor expression in human fascial tissue and modulation of the extracellular matrix according to the hormone levels

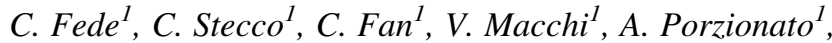 R. De Caro ${ }^{l}$}

${ }^{1}$ Department of Neuroscience, Institute of Human Anatomy, University of Padova, via Gabelli 65, 35121, Padova, Italy

Purpose: Many clinical and experimental findings point to sex differences in myofascial pain, demonstrating that adult women tend to have different myofascial problems with respect to men [1]. It is possible that sex hormones can play a role in extracellular matrix and collagen remodeling and thus contribute to functions of myofascial tissue, causing a sensitization of fascial nociceptors.

Methods: This study was approved by the Institutional Ethics Review Board according to ethical regulations regarding research conducted on human tissues. Immunohistochemical and molecular investigations of relaxin receptor 1 (RXFP1) and estrogen receptor-alpha $(\mathrm{ER} \alpha)$ were carried out on samples of human fascia collected from females volunteers patients during orthopedic surgery (age between 42 and 70 years, divided into preand post-menopausal groups), and in fibroblast cells isolated from deep fascia. Furthermore, an in vitro stimulation was performed with levels of beta-estradiol equal to the follicular phase or to the periovulatory phase, and the matrix was analyzed after Sirius Red staining.

Results: RXFP1 and ER $\alpha$ are expressed in all the human fascial districts examined and in fascial fibroblasts culture cells, to a lesser degree in the post-menopausal with respect to the pre-menopausal women. Furthermore, different levels of beta-estradiol modulate the collagen production, that increases when the hormone levels rise up to the periovulatory concentration $(\sim 400 \mathrm{pg} / \mathrm{mL})$. Our results demonstrated that the fibroblasts located within different districts of the muscular fasciae express sex hormone receptors and can modulate the extracellular matrix according to the hormone levels, influencing the tissue hydration and the lubrication of sliding surfaces.

Conclusions: These results can help to explain the link between hormonal factors and myofascial pain: estrogen and relaxin play a key role in extracellular matrix remodeling by inhibiting fibrosis and inflammatory activities, both important factors affecting fascial stiffness and sensitization of fascial nociceptors [2].

\section{References}

1. Rollman GB, Lautenbacher S (2001) Sex differences in musculoskeletal pain. Clin J Pain 17:20-24

2. Fede C, Albertin G, Petrelli L, Sfriso MM, Biz C, De Caro R, Stecco C (2016) Hormone receptor expression in human fascial tissue. Eur J Histochem 60(4):2710 


\section{BN1 O}

\section{Role of fasciae (around the median nerve) in carpal tunnel syndrome (pathogenesis): microscopic and ultrasound study}

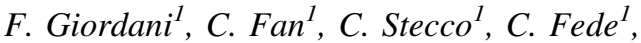 \\ A. Porzionato ${ }^{1}$, V. Macchi ${ }^{1}$, R. De Caro ${ }^{1}$ \\ ${ }^{1}$ Institute of Human Anatomy, Department of Neurosciences, \\ University of Padova, Via Gabelli 65, 35127 Padova, Italy
}

Purpose: Carpal Tunnel Syndrome (CTS) is the most common surgically treated problem in the hand affecting $1-3 \%$ of the population [1], while recurrence of CTS following surgery is reported to be between 7-20\% [2]. Accordance between nerve conduction studies and symptoms is often not found. Alternative pathogenesis have been proposed to explain those abnormalities. The aim of this study was to investigate connection between myofascial structure and epineural nerve sheaths under macroscopic and microscopic point of view in order to evaluate a possible role of fascia in CTS pathogenesis.

Methods: Anatomic study was carried out on 9 unembalmed upper arms. 4 samples of median nerve (MN) and surrounding structure have been taken for each arm at different levels for microscopic analysis. Ultrasound images of the MN were analyzed in 11 healthy subjects and 8 patients with CTS to evaluate the MN transversal displacement during the 3rd finger and all fingers motion at the Carpal tunnel (CT) and forearm level in all subjects.

Results: Anatomical continuity has been found between the epimysium and paraneural sheath and the reduction of paraneural fat tissue has been demonstrate from proximal to distal in all samples. Ultrasound study have shown a statistically significant reduction in MN transversal displacement in both sites between healthy and CTS patients $(\mathrm{p}<0.001)$. This study has clearly demonstrated a link between the MN sheaths and the muscular fasciae. Therefore, it is possible to argue that an unbalance condition of the epimysial fasciae has to be considered in the pathogenesis of the CTS. The distal segment of the arm will be more sensitive to pressure since the fat component reduction has been found from proximal to distal which reveals a decrease in the ability to cushioning to protect the nerve. Ultrasound study has confirmed the previous studies about reduction of nerve displacement in the $\mathrm{CT}$, but has also shown the reduction of nerve displacement in the forearm for the first time.

Conclusions: This new data suggests that the CTS is not just a nerve compression in the carpal tunnel, but has to be considered a global problem that can affect the nerve movements along all the upper limb. References

Atroshi I, Gummesson C, Johnsson R, Ornstein E, Ranstam J, Rosén I (1999) Prevalence of carpal tunnel syndrome in a general population. JAMA 282:153

Jones N, Ahn H, Eo S (2012) Revision surgery for persistent and recurrent carpal tunnel syndrome and for failed carpal tunnel release. Plast Reconstr Surg 129(3):683-692

\section{BN1 O \\ Lifelong football training effects on muscle cell-growth, proliferation and apoptosis pathways}

\author{
A. Mancini ${ }^{1,2}$, D. Vitucci ${ }^{3}$, M.B. Randers ${ }^{4}$, J.F. Schmidt ${ }^{4}$, \\ M. Hagman $^{4}$, T. Rostgaard ${ }^{4}$, E. Imperlini ${ }^{3}$, S. Orrü ${ }^{l, 3}$, \\ P. Krustrup ${ }^{4,5}$, P. Buono ${ }^{1,2,3}$
}

${ }^{I}$ Dipartimento di Scienze Motorie e del Benessere, Università

"Parthenope", Naples, Italy;

${ }^{2}$ CEINGE-Biotecnologie avanzate, Naples, Italy;

${ }^{3}$ IRCCS SDN Foundation, Naples, Italy;

${ }^{4}$ Copenhagen Centre for Team Sport and Health, Department of Nutrition, Exercise and Sports, University of Copenhagen, Copenhagen, Denmark;

${ }^{5}$ Health Sciences, College of Life and Environmental Sciences, St. Luke's Campus, University of Exeter, Exeter, UK

Purpose: Lifelong football training is associated with positive musculoskeletal and metabolic adaptations and reduced cardiovascular risk $[1,2]$. The present study is aimed to identify, through a differential transcriptomic approach, the effects of lifelong football training on the muscle expression of markers involved in cell-growth, proliferation and apoptosis pathways.

Methods: Total RNA was extracted from muscle biopsies of 6 veteran soccer players (VSP; aged 65-75) and 6 healthy old untrained men (CG; aged 65-75), provided by Copenhagen group; RNA integrity number (RIN) was determined. Single strand biotinylated cDNA was generated and hybridized to the Human Genechip HTA 2.0 Arrays (Affymetrix, Biogem Scarl, Ariano Irpino (AV). DAT files were obtained and results were filtered for fold change $>1.5$. Statistical analysis was performed using the ANOVA ( $p$ value 0.05 ).

Results: Preliminary processing showed that a total of $430(p<0.05)$ and $190(p<0.01)$ transcripts were identified as differentially expressed genes (DEGs) between VSP and CG. Among DEGs a 'focus gene' network was generated using Ingenuity Pathway Analysis (IPA). We found up-regulation of RAD23A, HSPB6, HSB1, Rab1B, TRAP1, SIRT2, correlated with proteasome subunits $20 \mathrm{~s}$ and $26 \mathrm{~s}$ and of RPL1, RPL4, RPL36, MRPL37 transcripts involved in cellular growth and proliferation in VSP muscle compared to CG, respectively. We also found up-regulation of key markers-Hsp70 and 90 - involved in the protein ubiquitination machinery and of Atg5-Atg12 and Bcl2 proteins, involved in the autophagy and apoptotic processes in VSP compared to CG muscle, respectively. We also evidenced down regulation of miR-1303 and miR-548 in muscles from VSP respect to CG.

Conclusions: Our preliminary results suggest that lifelong football training positively affects the expression of key markers, including miRNAs, involved in cell-growth and proliferation and in apoptosis pathways, that in turn are involved in healthy longevity pathways.

Acknowledgements: This work was supported by University of Naples Parthenope, "Bando per la ricerca individuale, annualità 2016 and 2017" and "Bando per la Ricerca competitiva, triennio 2016-2018 quota C".

References

1. Bangsbo J et al (2015) Br J Sports Med 49:568-576

2. Mancini et al (2017) Eur J Appl Physiol 117:721-730 


\section{MORPHOLOGICAL SCIENCES IN SPORT} AND EXERCISES

\section{MS K}

Morphological and volumetric analysis of the suprapatellar fat pad compared to infrapatellar fat pad in normal population and in osteoarthritis

\author{
V. Macchi ${ }^{1}$, C. G. Fontanella ${ }^{2}$, E. Belluzzi ${ }^{3}$, \\ E. E. E. Picardi ${ }^{1}$, G. Sarasin ${ }^{1}$, M. Rossato ${ }^{4}$, R. Ramonda ${ }^{5}$,

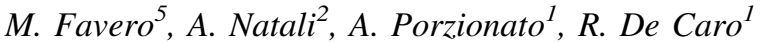 \\ ${ }^{1}$ Institute of Human Anatomy, University of Padova, Padova, Italy; \\ ${ }^{2}$ Department of Industrial Engineering, University of Padova, \\ Padova, Italy; \\ ${ }^{3}$ Musculoskeletal Pathology and Oncology Laboratory, Department \\ of Orthopedics and Orthopedic Oncology, University of Padova, \\ Padova, Italy: \\ ${ }^{4}$ Clinica Medica 3, Department of Medicine-DIMED, University \\ of Padova, Padova, Italy; \\ ${ }^{5}$ Rheumatology Unit, Department of Medicine-DIMED, University \\ Hospital of Padova, Padova, Italy
}

Purpose: The suprapatellar fat pad (SFP) is located above the patella and behind the suprapatellar joint recess with the function of increasing the congruency of the extensor mechanism. Osteoarthritis SFP has been demonstrated to produce high amount of inflammatory molecules and to be more fibrous than subcutaneous adipose tissue in osteoarthritis (OA) patients. The aim of this study was to analyze (1) the morphological characteristics of the SFP compared to that of the infrapatellar fat pad (IFP) in normal subjects; (2) the magnetic resonance (MR) volumetric characteristics of the IFP and the SFP in nonOA controls compared to moderate and end-stage OA patients.

Methods: Five specimens of SFP were sampled from bodies of the donation program of the University of Padova without history of OA. Forty-four MR images were collected: (a) 17 control; (b) 15 patients with moderate $\mathrm{OA}$; and (c) 12 patients with end-stage OA. Volume, depth, femoral and tibial arch lengths of IFP were quantified. The SFP volume, oblique, antero-posterior and, cranio-caudal lengths were determined.

Results: At histological examination, the SFP consisted of white adipose tissue, of lobular type, with lobules delimited by thin connective septa. In the radiological analysis a decrease of IFP volume, depth, femoral, and tibial arch lengths in moderate and end-stage OA compared to controls were observed. A difference in IFP hypointense signal was found between groups. No differences were found in SFP characteristics between the groups. In controls and moderate OA patients, correlations were found among the different MRI characteristics of both IFP and SFP, while in the end-stage OA group correlations were found only in SFP.

Conclusions: Differences of the IFP MRI morphometric characteristics between the groups analyzed supports an important role of IFP in OA pathology and progression. On the contrary, no differences were highlighted in SFP analysis suggesting that this fat pad is not clearly involved in OA, probably due to its peculiar localization and different function.

References

Schwaiger BJ, Mbapte Wamba J, Gersing AS, Nevitt MC, Facchetti L, McCulloch CE, Link TM (2018) Hyperintense signal alteration in the suprapatellar fat pad on MRI is associated with degeneration of the patellofemoral joint over 48 months: data from the Osteoarthritis Initiative. Skelet Radiol 47:329-339

Staeubli HU, Bollmann C, Kreutz R, Becker W, Rauschning W (1999) Quantification of intact quadriceps tendon, quadriceps tendon insertion, and suprapatellar fat pad: MR arthrography, anatomy, and cryosections in the sagittal plane. AJR Am J Roentgenol 173:691-698

\section{MS O}

The characteristics of the lobular arrangement support an active role of the infrapatellar fat pad in knee kinematics.

\author{
V. Macchi ${ }^{1}$, E. E. E. Picardi ${ }^{1}$, A. Porzionato ${ }^{1}$, C. Stecco ${ }^{1}$,

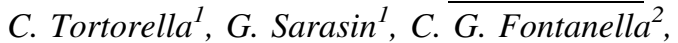 \\ A. Natali $^{2}$, R. De Caro ${ }^{1}$
}

${ }^{1}$ Institute of Human Anatomy, University of Padova, Padova, Italy; ${ }^{2}$ Department of Industrial Engineering, University of Padova, Padova, Italy

Purpose: The infrapatellar fat pad (IFP) is an intracapsular, but extrasynovial structure, located between the patellar tendon, femoral condyles and tibial plateau. It consists of white adipose tissue, organized in lobules delimited by thin connective septa. The aim of the study is the morphometric and ultrasonographic analysis of IFP in subjects without knee pathology during flexion-extension movements. Methods: The morphometric study was conducted on 20 cadavers (15M, 5F, mean age 80.2 years). Ultrasound was performed on 24 volunteers with no history of knee diseases $(5 \mathrm{M}, 19 \mathrm{~F}$, mean age: 45 years). The characteristics of the adipose lobules close to the patellar tendon and in the deep portion of the IFP were evaluated. Numerical models were provided considering the dimensions of the lobules.

Results: At histological examination, the adipose lobules located close to the patellar tendon were larger (mean area $12.2 \mathrm{~mm}^{2}$ ) with respect to the lobules located at deep level (mean area $1.34 \mathrm{~mm}^{2}$, $\mathrm{p}<0.001$ ), and the thickness of the septa of the deepest adipose lobules (mean value $0.35 \mathrm{~mm}$ ) was major than that of the superficial one (mean value $0.29 \mathrm{~mm}, \mathrm{p}<0.001$ ). At ultrasound, the IFP was constituted by very large lobules in the superficial part (mean area $0.29 \mathrm{~cm}^{2}$ in extension), with a significant reduction in flexion movement (mean area $0.12 \mathrm{~cm}^{2}, \mathrm{p}<0.01$ ). The deep lobules were smaller (mean area $0.11 \mathrm{~cm}^{2}$ in extension) and did not change their values (mean area $0.19 \mathrm{~cm}^{2}$ in flexion, $\mathrm{p}>0.05$ ). The reduction of thickness of the superficial layer with the large adipose lobules during flexion was $20.6 \%$, whereas those of the deep layer with small adipose lobules was $1.3 \%$. With numerical simulation of vertical load, corresponding to flexion, the stress mainly developed within interlobular septa and opposed bulging of the adipose lobules.

Conclusions: The characteristics of the lobular arrangement of the IFP (large lobules with thin septa in the superficial part and small lobules with thick septa in the deep part), the significant change in area and perimeter of the superficial adipose lobules and the reduction of the thickness of the superficial layer during flexion, support an active role of IFP in knee kinematics.

\section{References}

Macchi V, Porzionato A, Sarasin G, Petrelli L, Guidolin D, Rossato M, Fontanella CG, Natali A, De Caro R (2016) The infrapatellar adipose body: a histotopographic study. Cells Tissues Organs 201:220-231

Vera-Pérez E, Sánchez-Bringas G, Ventura-Ríos L, Hernández-Díaz C, Cortés S, Gutiérrez M, Pineda C (2017) Sonographic characterization of Hoffa's fat pad. A pilot study. Rheumatol Int 37:757-764 


\section{MS O}

\section{Beneficial effects of exercise and olive oil-enriched diet} on musculoskeletal and liver disorders

\section{N. A. Szychilinske, G. Musumeci}

Department of Biomedical and Biotechnological Sciences, Human Anatomy and Histology Section, School of Medicine, University of Catania, Catania, Italy

Purpose: The beneficial effects of Extra Virgin Olive Oil (EVOO), the main source of culinary and dressing fat of Mediterranean diet, have been, and still are, widely studied thanks to its anti-inflammatory and antioxidant properties. Lubricin is a chondroprotective glycoprotein, serving as a critical boundary lubricant between opposing cartilage surfaces. A joint injury causes an increased cytokine expression, which is associated with decreased lubricin production and predisposes to cartilage degeneration, leading to osteoarthritis. The aim of this study was to evaluate the beneficial role of EVOOenriched diet and physical activity on cartilage, muscle and liver tissues, in rats after injury represented by anterior cruciate ligament transection (ACLT) to induce osteoarthritis.

Methods: To this purpose, we performed histomorphometric, histological, immunocytochemical, immunohistochemical, western blot and biochemical analysis for lubricin and interleukins evaluations in articular cartilage and synovial fluid of rats and by histology the muscle and liver morphology evaluations.

Results: The results showed the beneficial effect of physical activity (treadmill training) and EVOO supplementation on the rat articular cartilage. ACLT determined an increase in interleukins expression and a significant decrease in the lubricin expression, while physical activity and EVOO supplemented diet, determined that the values returned to a normal level when compared to the control group. In conclusion, the results showed a beneficial effect of the conjunction of EVOO-based diet, corresponding to the Mediterranean diet, and physical activity on the preservation of articular cartilage tissue.

Acknowledgments: This study was supported by the University Research Project Grant, University of Catania, Italy.

Reference

Musumeci G, Trovato FM, Pichler K, Weinberg AM, Loreto C, Castrogiovanni P (2013) Extra-virgin olive oil diet and mild physical activity prevent cartilage degeneration in an osteoarthritis model: an in vivo and in vitro study on lubricin expression. $\mathrm{J}$ Nutr Biochem 24(12):2064-2075

Keywords: Extra virgin olive oil, Physical activity, Cartilage, Lubricin, Osteoarthritis

\section{MS O \\ The effects of mechanobiology on MSC-derived articular cartilage}

\section{G. Musumeci}

Department of Biomedical and Biotechnological Sciences, Human Anatomy and Histology Section, School of Medicine, University of Catania, Catania, Italy

Aim: Mesenchymal stem cells (MSCs) are currently being investigated as a cell source for regenerative medicine approaches for the repair of damaged articular cartilage. It is important to understand how these cells react to the complex loading environment of a joint in vivo, to use them as a source for the cell-based therapy for articular cartilage regeneration. In addition to investigate alternative MSC sources, it is also important to study the structure of tissue-engineered constructs and their organization within them.

Methods: A custom-built bioreactor was used to expose human MSCs to a combination of shear and compression loading. The MSCs were either evenly distributed throughout fibrin-poly(ester-urethane) scaffolds or asymmetrically seeded with a small proportion seeded on the surface of the scaffold. The effect of cell distribution on the production and deposition of cartilage-like matrix in response to mechanical load mimicking in vivo joint loading was then investigated.

Results: The results showed that asymmetrically seeding the scaffold led to markedly improved tissue development based on histologically detectable matrix deposition. Consideration of cell location, therefore, is an important aspect in the development of regenerative medicine approaches for cartilage repair. This is particularly relevant when considering the natural biomechanical environment of the joint in vivo and patient rehabilitation and adapted physical activity protocols.

Acknowledgements: This study was supported by the University Research Project Grant (Triennial Research Plan 2016-2018), University of Catania, Italy.

\section{Reference}

Gardner OFW, Musumeci G, Neumann AJ, Eglin D, Archer CW, Alini M, Stoddart MJ (2017) Asymmetrical seeding of MSCs into fibrin-poly(ester-urethane) scaffolds and its effect on mechanically induced chondrogenesis. J Tissue Eng Regen Med 11(10):2912-2921 Keywords: MSC, Articular cartilage, Bioreactor, Chondrogenesis, Mechanobiology

\section{MS O Modification of skeletal muscle homeostasis after an acute bout unaccustomed exercise}

\author{
K. Myburgh ${ }^{1}$, A. Isaacs ${ }^{1}$, F. Farina ${ }^{2}$, \\ F. Cappello ${ }^{2,3}$, V. di Felice ${ }^{2}$, \\ F. Macaluso ${ }^{2,3,4}$
}

${ }^{1}$ Department of Physiological Sciences, Stellenbosch University, Stellenbosch, South Africa;

${ }^{2}$ Department of Experimental Biomedicine and Clinical

Neurosciences (BioNeC), University of Palermo, Italy;

${ }^{3}$ Euro-Mediterranean Institute of Science and Technology (IEMEST), Italy;

${ }^{4}$ SMART Engineering Solutions and Technologies (SMARTEST)

Research Center, eCampus University, Italy

Purpose: Plyometrics refers to exercise that exploits the stretchshortening cycle, which proceeds with the rapid stretch of a muscle (eccentric phase), followed by rapid shortening of the same muscle (concentric phase), which results in increased force and power output of the activated muscles. This type of training has been successfully used in different sporting contexts to improve strength, muscle power, coordination, and athletic performance. Studies investigating the effect of an acute bout of plyometric exercise or the regular plyometric training on muscle morphology are limited.

Methods: Skeletal muscle biopsies of healthy untrained volunteers have been observed for structural and ultrastructural change induced by an acute bout of plyometric exercise $(10 \times 10$ squat-jumps, 1 -min rest) or plyometric training (3 times per week for 8 weeks). 
Results: The results indicate that an acute bout of plyometric exercise mainly affected the fast-twitch muscle fibers (Type II muscle fibers), damaging both the sarcolemma and the sarcomere at the site of the Z-disk. While the plyometric exercise in persons accustomed to eccentric exercise (trained for 8 weeks) does not damage the sarcolemma and the sarcomere at the site of the Z-disk.

Conclusion: Athletic trainers should avoid prescribing high-volume plyometric exercise bouts within quick succession or after other forms of high-intensity exercise that are known to stress the fast-twitch muscle fibers, so that athletes have sufficient time to regenerate damaged fibers.

\section{References}

Macaluso F, Isaacs AW, Di Felice V, Myburgh KH (2014) Acute change of titin at mid-sarcomere remains despite $8 \mathrm{wk}$ of plyometric training. J Appl Physiol (1985) 116(11):1512-1519

Macaluso F, Isaacs AW, Myburgh KH (2012) Preferential type II muscle fiber damage from plyometric exercise. J Athl Train 47(4):414-420

\section{SPORT BIOMECHANICS AND TECHNOLOGY}

\section{SB2 K \\ Fat content and muscle quality of the quadriceps muscle assessed by electrical impedance myography: correlations with ultrasound measurements}

\section{S. Longo, Emiliano Cè, A.V. Bisconti, S. Rampichini, E. Limonta, S. Shokoyar, L. Galasso, G. Coratella, F. Eposito}

Department of Biomedical Sciences for Health, Università degli studi di Milano, Milano, Italy

Purpose: Electrical Impedance Myography (EIM) has been proposed as a non-invasive approach for the assessment of fat content (FM) and muscle quality (MQ) [1]. A new portable device (Skulpt ${ }^{\circledR}$ Chisel) has been developed to estimate these parameters. The device is portable, battery-operated, and connected to a smartphone via Bluetooth ${ }^{\circledR}$. The present study aimed to assess FM and MQ in a population of healthy young participants and to correlate them with FM and MQ measured by ultrasound.

Methods: Twenty-five participants $(12 \mathrm{M}, 13 \mathrm{~F}$; mean $\pm \mathrm{SD}$ : age $=$ $24 \pm 4.3$ years; $\quad$ stature $=1.71 \pm 0.09 \mathrm{~m} ; \quad$ body $\quad$ mass $=63.0 \pm$ $10.0 \mathrm{~kg}$ ) underwent two EIM measurements over the quadriceps muscle at $50 \%$ of the femur length. FM and MQ were assessed by Skulpt (Skulpt ${ }^{\circledR}$ Chisel). EIM involved high-frequency $(50 \mathrm{kHz})$, lowintensity electrical alternating current applied to the muscle belly using 12 surface electrodes. Thereafter, the same area was scanned by ultrasound in the transverse plane. Six snapshots were taken with the same brightness and contrast parameters. Subcutaneous fat, muscle thickness (FT and MT, respectively) and echo intensity as muscle quality index (MQei, grey scale) were measured offline as average of 6 measures. EIM and ultrasound parameters were then correlated.

Results: Significant correlations were found between: FM and FT $(\mathrm{r}=0.94, \mathrm{p}<0.01)$, FM and MQei $(\mathrm{r}=0.53, \mathrm{p}<0.01)$, and MQ and MQei $(r=0.47, p<0.05)$. No correlation was found between MQ and MT $(\mathrm{r}=0.29, \mathrm{p}>0.05)$.
Conclusions: FM and MQ assessed by EIM were correlated to subcutaneous FT and MQei obtained by ultrasound. Therefore, it seems that Skulpt can provide an index of fat percentage and muscle quality within the area of measurement in a quick and cheap manner. Further studies are needed to assess these correlations in other muscles and to check the applicability of Skulpt in monitoring training-induced changes in FM and MQ at local level.

\section{Reference}

1. Rutkove SB (2009) Electrical impedance myography: background, current state, and future directions. Muscle Nerve 40(6):936-946

\section{SB2 O \\ Discrepancies in glycaemia values during exercise sessions at different intensities}

\author{
M. Vandoni ${ }^{1}$, V. Carnevale Pellino ${ }^{1}$, L. Correale ${ }^{1}$, \\ E. Ricagno $^{1}$, M. Bleve ${ }^{1}$, O.E. Ferraro ${ }^{2}$, V. Natalucci ${ }^{3}$, \\ S. Bonfadini ${ }^{4}$, E. Cimino ${ }^{4}$, A. Girelli ${ }^{4}$ \\ ${ }^{1}$ Laboratory of Adapted Motor Activity (LAMA), Department \\ of Public Health, Experimental Medicine and Forensic Science, \\ University of Pavia, Pavia, Italy; \\ ${ }^{2}$ Unit of Biostatistics and Clinical Epidemiology, Department \\ of Public Health, Experimental and Forensic Medicine, University \\ of Pavia, Pavia, Italy; \\ ${ }^{3}$ Department of Biomolecular Science (DISB), Carlo Bo University \\ of Urbino, Urbino, Italy; \\ ${ }^{4}$ U.O di Diabetologia ASST Spedali Civili, Brescia, Brescia, Italy
}

Purpose: The continuous measurement of interstitial glucose through a sensor placed in the subcutaneous tissue is becoming increasingly widespread for patients on intensive insulin therapy. The possibility of evaluate in real time not only the value but above all the glucose trend has proved to be effective in improving glycaemic control, in reducing hypoglycaemic risk and in improving patient QoL. One of the most debated points is the accuracy of the data detected by the sensor. Patients often report that the measurements of glycaemic by sensor during physical activity, in particular in high intensity exercise, presents too different values compared to the measurement from capillary. The aim of the study is to evaluate the difference in the interstitial glucose determination compared to the reference value (capillary blood sugar) during different type and intensity of exercise, in particular how this value different from rest condition.

Methods: 20 subjects with type 1 diabetes have been recruited for a 3-day educational camp focused on physical activity (PA) and management of glycaemia. For this reason, subjects wore heart-rate monitor during exercise sessions and recorded glycaemic values both with sensor and capillary measurement.

All subjects performed activities at different tasks: low intensity walking, moderate to vigorous intensity jogging and high intensity uphill running for $20 \mathrm{~min}$ each and a mixed-intensity activity (mountain hike) for $6 \mathrm{~h}$. The operator collected heart-rate monitor values and the glycaemic measurement before, during and after every training session. Qualitative variables are summarized as percentages and quantitative variables as mean and standard deviation. Concordance in the two measurements of glycaemic were tested using a nonparametric method (Kendall's test).

Results: All the subjects ( 12 women, $46.4 \pm 13.5$ years; mean $\pm \mathrm{ds}$ ) successfully completed the training sessions. During every task recorded capillary and sensor values were discordant $(\mathrm{p}<0.05)$. Glycaemic values from both sensor and capillary changed in accordance to exercise intensity. 
Conclusions: This study demonstrated that during exercise sessions on field there is a discrepancy between sensor and capillary glycaemia values. This phenomenon could lead to bias and especially during hypo and hyperglycaemia it must be carefully evaluated to carry values in a safe condition.

Reference

Yardley JE, Sigal RJ, Kenny GP, Riddell MC, Lovblom LE Perkins BA (2013) Point accuracy of interstitial continuous glucose monitoring during exercise in type 1 diabetes. Diabetes Technol Ther 15(1). https://doi.org/10.1089/dia.2012.0182

\section{SB2 O}

The effects of land and aquatic treadmill running at moderate intensities on heart rate and postural balance control

\author{
A. Rizzato $^{1}$, G. Bosco ${ }^{1}$, G. Zorzetto ${ }^{1}$, G. Marcolin ${ }^{1}$, \\ ${ }^{1}$ Department of Biomedical Sciences, University of Padova, Italy
}

Purpose: An impairment of postural balance control has been shown after land treadmill (LTM) running, due to a disturbance of vestibular and visual information but no studies are available on aquatic treadmill (ATM). Therefore, the aim of our study was to investigate the effects of running at moderate intensity over ATM and LTM on the postural balance control both with open eyes (OE) and closed eyes (CE).

Methods: Center of Pressure (COP) trajectory of 20 subjects was collected on a dynamometric platform before (PRE) and immediately after (POST) a 20-min-long moderate running (5/10 of Borg's scale) on ATM and LTM. Heart rate (HR) was recorded every 30 s. Subjects performed 5 trials in both $\mathrm{OE}$ and CE condition. Each trial lasted $30 \mathrm{~s}$. Besides classical parameters (sway path, sway area, area of the confident ellipse, and maximal oscillations) the sample entropy (SampEn) was calculated to assess the regularity of the COP time series.

Results: HR was significantly lower in the ATM with respect to LTM. In the OE condition, statistically significant differences were detected only for maximal medio-lateral (ML) oscillations in ATM, with an increase in the POST condition. For the LTM running the maximal ML oscillations and the sway area resulted to be higher in the POST condition.

In the CE condition, on the LTM a higher SampEn after running was observed. Two-way ANOVA for repeated measures revealed a significant main effect of eyes condition for all classical parameters and a main effect of treadmill only for ML SampEn.

Conclusions: Postural balance control was similarly influenced by ATM and LTM running. However, the main cause of postural balance control perturbation was given by the deprivation of visual information. The lower SampEn in the CE condition corresponded to a reduced complexity of the physiological and behavioral systems involved in the task.

References

Derave W, Tombeux N, Cottyn J, Pannier JL, De Clercq D (2002) Treadmill exercise negatively affects visual contribution to static postural stability. Int J Sports Med 23:44-49

Ramdani S, Seigle B, Lagarde J, Bouchara F, Bernard PL (2009) On the use of sample entropy to analyze human postural sway data. Med Eng Phys 31(8):1023-1031

\section{SB2 O}

The use of a mobile application to support physical activity and lifestyle changes in persons living with HIV: the smartapp study

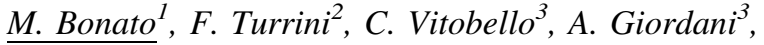 \\ A. Meloni $^{1}$, L. Galli ${ }^{2}$, A. La Torre ${ }^{1}$, G. Merati ${ }^{1,4}$, \\ P. Cinque $^{2}$
}

${ }^{1}$ Department of Biomedical Sciences for Health, Università degli Studi di Milano, Milan, Italy;

${ }^{2}$ Department of Infectious Disease, San Raffaele Scientific Institute, Milan, Italy;

${ }^{3}$ U.O. Riabilitazione Specialistica Disturbi Neurologici CognitiviMotori, San Raffaele Scientific Institute, Milan, Italy;

${ }^{4}$ Centre of Sport Medicine, Don Carlo Gnocchi Foundation, Milan, Italy

Purpose: Although physical activity has been shown to improve fitness, metabolic and inflammatory parameters in people living with HIV, adherence to exercise programs is usually low when not strictly supervised. Therefore, the aim of this study was to assess if the use of a mobile application will favor engagement to exercise by providing motivational inputs, and therefore adherence to training. We hypothesized, as a consequence an improvement of physical fitness, and therefore of health status.

Methods: This study was a two-armed, parallel group, randomized controlled trials in which HIV-infected subjects were enrolled and assigned to either an experimental group (EG), which trained with a smartphone application, or a control group (CG), which trained with a hard copy training program. Physical activity program included an initial coach-supervised period of 4 weeks, followed by 12 weeks where participants were instructed to train independently. The program consisted of walking or jogging three times/week for $60 \mathrm{~min}$. At baseline (BL) and after 16-weeks (W16), patients underwent measurement of CRF by peak oxygen consumption $\left(\mathrm{VO}_{2 \text { peak }}\right)$, body composition (body mass, body mass index-BMI, waist, hip circumferences, \%fat mass and \%fat free mass by bioimpedentiometry), and metabolic parameters (total-, HDL-, LDL-cholesterol). Results are given as number (\%) or median (IQR) values. Intention-to-treat analysis regarding an improvement of the $15 \%$ of $\mathrm{VO}_{2 \text { peak }}$ was performed with a Chi square test. Percentage changes between BL and W16 regarding EG and CG were assessed by Wilcoxon matched-pairs signed rank test.

Results: Thirty-seven subjects were screened and 28 were eligible and divided in EG [ $\mathrm{n}=13$, age: $52(36-56) \mathrm{y}-\mathrm{o}$, height: $176(170-180) \mathrm{cm}$, BMI: $\left.24.4(22.1-27.1) \mathrm{kg} / \mathrm{m}^{2}, \mathrm{CD}^{+}: 648(439-762)\right]$, and CG $[\mathrm{n}=15$, age: 50 (36-56) y-o, height: 172 (170-180) cm, BMI: 24.8 (22.1-27.1) $\mathrm{kg} / \mathrm{m}^{2}, \mathrm{CD}^{+}: 619$ (439-762)]. During training we had 1 and 2 dropouts for EG and CG, respectively. Median training adherence was $100 \%$ (IQR 91-100\%) during the initial coach supervised period, and 70\% (IQR 41-91\%) during the independently training period, with $15 / 28$ (54\%) who trained for at least $50 \%$ of the sessions. Intention-to-treat analysis showed post-intervention improvement in $\mathrm{VO}_{2 \text { peak }}(+15 \%$, $\mathrm{p}=0.005), \%$ fat mass $(-10 \%, \mathrm{p}=0.014) ; \%$ fat free mass $(+8 \%$, $\mathrm{p}=0.008)$, total cholesterol $(-6 \%, \mathrm{p}=0.016)$ between EG and CG were observed. Adherence to training is fundamental but seems to be low when not strictly supervised in HIV infected patients. These results showed that patients trained using a smartphone application were more adherent and as consequence improved physical fitness, body composition and metabolic parameters.

\section{Reference}

Bonato M et al (2017) BMC Infect Dis

Keywords: Digital health, exercise tools, exercise training, HIV infection. 


\section{EXERCISE AND SPORT PSYCHOLOGY 1}

\section{SP1 K \\ Imagery ability in sport types: differentiation between vividness and kinesthetic imagery, and mental rotation}

\section{$\underline{\text { D. Di Corrado }}{ }^{1}$, M. Guarnera ${ }^{2}$, F. Vitali $^{3}$, A. Quartiroli $^{4}$,} M. Coco $^{5}$

${ }^{1}$ Department of Sport Sciences, Kore University, Enna, Italy; ${ }^{2}$ Department of Psychology, Kore University, Enna, Italy; ${ }^{3}$ Department of Neurological and Movement Sciences, University of Verona, Italy;

${ }^{4}$ Department of Psychology, University of Wisconsin, USA; ${ }^{5}$ Department of Biomedical and Biotechnological Sciences, University of Catania, Italy

Purpose: In a sports context, imagery has been described as the condition in which persons imagine themselves in habits which may lead to increasing and performing skills. Systematic reviews have shown that mental imagery improves performance in motor tasks. The aim of the present study was to explore whether type of sport (team open-skill sports, individual open-skill sports, and individual closedskill sports) influences static and dynamic vividness (i.e., the clarity or realism of the imagery experience) and the controllability of mental images (i.e., the ease and accuracy with which an image can be manipulated mentally) differently.

Methods: The study was conducted with volunteers comprising 30 tennis players, 30 golfers, 30 handball athletes, 30 rugby athletes, and 30 non-athlete students, between the ages of 14 and 33 years $(M=20.4, S D=4.56)$. The participants completed the Vividness of Visual Imagery Questionnaire, The Vividness of Movement Imagery Questionnaire-2, and Mental Rotation Tasks.

Results: Results showed that competitive athletes have higher scores on imagery ability than control group. Pairwise post hoc comparisons using Bonferroni tests showed significantly lower scores in the control group on External Visual Imagery, Internal Visual Imagery and Kinesthetic Imagery (tennis players vs control $p<.001$ ). Volleyball and rugby athletes demonstrated significantly highest scores of Kinesthetic Imagery, than the other groups. Results shown that the sport type may lead to differences in the imagery ability.

\section{References}

Moreau D, Clerc J, Mansy-Dannay A, Guerrien A (2010) Assessing movement imagery ability: self-report questionnaires vs. performance-based tests. Eur J Psychol 4:93-109

Watt AP, Spittle M, Morris T (2002) Evidence related to the evaluation of measures of sport imagery. J Sci Med Sport 5:29

Guillot A, Collet C (2010) The neurophysiological foundations of mental and motor imagery. Oxford University Press, Oxford

Keywords: Imagery, Sport types, Mental rotation, Motor tasks, Performance

\section{SP1 O}

Effect of mental fatigue on physical and technical performance in young soccer players

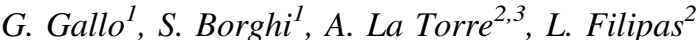

${ }^{1}$ School of Sport Sciences, Università degli Studi di Milano, Milan, Italy; ${ }^{2}$ Department of Biomedical Sciences for Health, Università degli Studi di Milano, Milan, Italy; ${ }^{3}$ IRCCS Istituto Ortopedico Galeazzi, Milan, Italy

Purpose: The aim of this study was to investigate the effects of mental fatigue on physical and technical performance in three different age groups of young soccer players.

Methods: Single blind cross-over randomised counterbalanced design. Subjects: 36 young soccer players, divided in $3 \times 12$ groups based on their age (G1: 13-14 years, G2: 15-16 years, G3: 17-18 years). The first part of the study assessed soccer-specific physical performance of the subjects using the Yo-Yo Intermittent Recovery Test, Level 1 (Yo-Yo IR1). The second part of the study assessed soccer-specific technical performance of the subjects using the Loughborough Soccer Passing and Shooting Tests (LSPT, LSST). Each test was performed after two different treatments: 30 min of the Stroop task (Mental Fatigue Condition) or 30 min reading magazines (Control Condition). Distance run, heart rate, and ratings of perceived exertion were recorded during the Yo-Yo IR1. LSPT performance time was calculated as original time plus penalty time. LSST performance was assessed using shot speed, shot accuracy, and shot sequence time. Results: The running distance in the Yo-Yo IR1 was reduced in G1, G2 and G3. No difference in heart rate existed between conditions, whereas ratings of perceived exertion were significantly higher at the same time of the test in the mental fatigue condition. LSPT original time, penalty time and performance time were reduced only in G3. Shot speed, shot accuracy and shot sequence time were similar between conditions in G1, G2 and G3.

Conclusions: Mental fatigue impairs soccer-specific physical performance in the three different age groups, whereas passing impairs only in the older group. Mental fatigue did not alter shot performances.

\section{Reference}

Smith MR, Coutts AJ, Merlini M, Deprez D, Lenoir M, Marcora SM (2016) Mental fatigue impairs soccer-specific physical and technical performance. Med Sci Sports Exerc 48(2):267-276

\section{SP1 O}

\section{Improved tolerance to mental exertion after 4 weeks of endurance training}

L. Filipas $^{1}$, A. La Torre
B. Rattray
${ }^{3}$

${ }^{I}$ Department of Biomedical Sciences for Health, Università degli Studi di Milano, Milan, Italy;

${ }^{2}$ IRCCS Istituto Ortopedico Galeazzi, Milan, Italy;

${ }^{3}$ University of Canberra Research Institute for Sport and Exercise, Canberra, Australia

Purpose: The aim of the study was to investigate if 4 weeks of endurance training could improve tolerance to mental exertion and cognitive performance in untrained individuals.

Methods: Twenty untrained individuals were allocated randomly in a training (TG) or control group (CG). Participants in both groups completed three testing visits before and after a 4-weeks endurance training program (or control program for $\mathrm{CG}$ ). The first testing session 
was a $\mathrm{VO}_{2}$ max test to assess the fitness level of the subjects. The other two visits included a cognitive activity (a battery of different cognitive tests or a control condition) followed by a 15-min time-trial performed on the cycle ergometer. Subjective ratings of mental fatigue, mental effort and motivation were measured before and after treatment. Power, cadence, speed, heart rate and perception of effort were measured during time-trial.

Results: In both groups mental fatigue impaired significantly performance on the time trial during the pre-tests. TG improved significantly the $\mathrm{VO}_{2}$ max after the training program, while $\mathrm{CG}$ did not change from preto post-tests. Post-tests performance on the time-trial was significantly improved in TG, whereas not in CG. In TG mental fatigue did not impair time-trial performance in post-tests; in CG mental fatigue reduced significantly time-trial performance also in post-tests.

Conclusions: Four weeks of endurance training improved tolerance to mental exertion in untrained individuals.

Reference

Van Cutsem J, Marcora S, De Pauw K, Bailey S, Meeusen R, Roelands B (2017) The effects of mental fatigue on physical performance: a systematic review. Sports Med 47(8):1569-1588

\section{SP1 O}

\section{Postural control and stress in young men}

\section{Coco ${ }^{1}$, C. Savia Guerrera ${ }^{2}$, S. Di Nuovo ${ }^{2}$, A. Buscemi $^{3}$, D. Di Corrado 4}

\author{
${ }^{1}$ Department of Biomedical and Biotechnological Sciences, \\ University of Catania, Italy; \\ ${ }^{2}$ Department of Educational Sciences, University of Catania, Italy; \\ ${ }^{3}$ Department of Research, Italian Center Studies of Osteopathy, \\ Catania, and Horus Cooperative Social, Ragusa; \\ ${ }^{4}$ Kore University of Enna, Italy
}

Purpose: It has been observed that the amount of stress influences postural stability in young women. The purpose of this study was to analyze whether enhanced stress may negatively influence the postural control in 14 young right-handed men during quiet standing with and without visual input.

Methods: Amount of stress was assessed by evaluating free cortisol response to awakening (Cortisol Awakening Response, CAR) and by administering the Perceived Stress Scale (PSS), whereas the Profile of Mood States (POMS) was utilized to evaluate total distress. The postural control was assessed with a force platform by calculating $95 \%$ confidence ellipse area occupied by the Center of Pressure throughout five stability positions maintained for at least $52 \mathrm{~s}$ with open and closed eyes. Results: A strong significant positive relation between POMS, PSS and CAR for every studied participants was observed. Moreover, it has been detected not only that the amount of stress influences postural stability, but also that this effect is influenced by laterality. In fact, it was detected that the ability to maintain the position is greater when the dominant foot is forward, irrespective of visual input.

\section{References}

1. Doumas M, Morsanyi K, Young WR (2018) Cognitively and socially induced stress affects postural control. Exp Brain Res 236(1):305-314

2. Demirovic D, Rattan SI (2013) Establishing cellular stress response profiles as biomarkers of homeodynamics, health and hormesis. Exp Gerontol 48:94-98

3. Jorgensen MG, Rathleff MS, Laessoe U et al (2012) Time-of-day influences postural balance in older adults. Gait Posture 35:653-665 Keywords: Stress, Postural control, Salivary cortisol, Gender, Laterality
SUNDAY POSTER SESSION

PHYSICAL EDUCATION AND SPORT PEDAGOGY

\section{PE P}

\section{Attitudes toward inclusion and perception of disability in Sport Sciences students}

\section{P. Oliva, A. M. Murdaca,}

Department of Cognitive Sciences, Psychology, Educational and Cultural Studies, University of Messina

Purpose: The inclusion process requires that the educational contexts are identified with certain principles and attitudes which teachers/coaches are consistent with. Although many children with disabilities can have access to regular schools or sport teams, in many cases they are exempted from physical activity, often for insecurity by the part of the teacher/coach (Hutzler and Barak 2017; Taliaferro et al. 2015; Tant and Watelain 2016). The purpose of this study was to analyze the attitudes toward inclusion and perception of disability of university Sport Sciences students. This study also aimed to compare the perceptions of different level students (1st Level Degree vs. 2st Level Degree).

Methods: For that, 101 university Sport Sciences students, of which 67 were 1 st Level Degree students and 34 were 2st Level Degree students completed: SACIE-R (Sentiments, Attitudes, and Concerns about Inclusive Education Revised), TAIS (Teacher Attitudes Toward Inclusive Education Scale) and an adapted version of TIAQ (Teacher Inclusion Attitudes Questionnaire).

Results: Results found that attitude toward inclusion was related to student competence perception (self-efficacy); furthermore, it was observed that 2nd Level Degree students reported more positive attitudes toward inclusion, even if they showed concerns about disability. Stronger students pessimism was about their lack of preparing to work with disabled children.

Conclusions: These findings underscore the need for improved student education to meet the needs of all children able to participate in youth sports.

References

Hutzler Y, Barak S (2017) Self-efficacy of physical education teachers in including students with cerebral palsy in their classes. Res Dev Disabil 68:52-65

Taliaferro AR, Hammond L, Wyant K (2015) Preservice Physical Educators' self-efficacy beliefs toward inclusion: the impact of coursework and practicum. Adapt Phys Activ Q 32:49-67

Tant M, Watelain E (2016) Forty years later, a systematic literature review on inclusion in Physical Education (1975-2015): a teacher perspective. Educ Res Rev 19:1-17 


\section{PE P}

\section{A new definition of Physical Activities significant for development and health (work in progress ...)}

\section{Lanza}

Department of Neurosciences, Biomedicine and Movement, University of Verona

Purpose: Physical activities that have been shown to be significant for development and health are not correctly represented by the famous classification of Corbin and colleagues (2000). The aim of research is to produce a complete and coherent definition of physical activities that are significant for health and development according to the kinesiology's literature. The classification could favour the correct promotion of physical activity in different ages and conditions.

Methods: Initially, a bibliographic survey (rapid review) concerning the definitions of physical activity was carried out. Having noted the lack of a comprehensive definition of the different types of physical activity that scientific research indicates as significant for development and health, a second rapid review was carried out on the types of physical activities significant for the promotion of health, the prevention of pathologies and the development of human beings. From the results, we proceeded to formulate a classification with a description of physical activities. The classification underwent a preliminary verification through two focus groups with students of the degree and master course in sport sciences.

Results: The proposed classification is the following: Sport: Competitive physical activity carried out within a system of rules defined by formal organizations and aimed at performance. Expressive physical activity: Activity carried out within an organized system aimed at the search for high performance in self expression. Professional Physical Activity: Jobs that require light, moderate or vigorous physical exertion and different from sports and expressive motor activities. Physical Exercise: Structured motor activity that aims at benefits to improve physical fitness, well-being and health. Recreational Physical Activity/Physical Activity practiced by passion: Unstructured motor activity, carried out with the prevailing motivation of personal pleasure. Physical Activity of Daily Life: Physical activity carried out with the main motivation to realize the functions and commitments of daily life outside of work.

Discussion: The classification must be now submitted to validation with experts who express, at least, a construct-related validity.

\section{PE $P$}

\section{Physical activity levels and motor performances of adolescents. Preliminary study for the promotion of physically active lifestyles}

\section{Colella ${ }^{1}$, D. Monacis ${ }^{1}$, C. d'Arando ${ }^{1}$ \\ ${ }^{1}$ Department of clinical and experimental medicine, Laboratory of didactic of motor activities, University of Foggia, Foggia, Italy}

Purpose: Many adolescents in different countries do not respect international guidelines for daily physical activity. In the developmental age, sedentary habits are among the fundamental causes of overweight and obesity, low levels of physical activity and motor performance. The aim of the study is to evaluate and compare the levels of physical activity and motor performance in two groups of high school students, divided according to gender and group differences (Male-nw $\mathrm{n}=10$, age $17.6 \pm 0.55$, BMI $21.59 \pm 2.13$; Male-ow/ob n $=10$; age $17.6 \pm 0.54$, BMI $26.04 \pm 1.03$; Female-nw $\mathrm{n}=10$, age $17.6 \pm 0.54$, BMI $21.42 \pm 1.53$; Female-ow/ob $\mathrm{n}=10$, age $16.8 \pm 0.83$, BMI $25.08 \pm 0.55$ ).

Methods: All the students identified, not practicing extracurricular sports, wore a pedometer for 7 days (Michalopoulou et al. 2011; Pangrazi et al. 2007). Self-report PAQ_A (Janz et al. 2008), standing long jump, shuttle run $10 \times 5$, sit up, medicine ball throw $(\mathrm{kg} 2)$ were assessed.

Results: In addition to the descriptive statistics ( $\mathrm{M} \pm \mathrm{DS}$ ), ANOVA 2 (group) $\times 2$ (gender) was carried out, in order to highlight significant differences between the variables studied $(p<0.05)$. Males show higher motor performance than females in all motor tests $(\mathrm{p}<0.05)$ except for the medicine ball throw test $(\mathrm{kg} 2)$. Males-nw and females-nw show higher motor performance than the ow/ob group $(\mathrm{p}<0.05)$. The results underline intra-group differences, nw vs ow-ob, in the levels of physical activity measured with the pedometer $(\mathrm{p}<0.05)$ and with the self-report $(\mathrm{p}<0.05)$.

Conclusions: BMI influences the levels of physical activity and the development of motor skills of the adolescent. Evaluation with objective measures and self-report is necessary to promote the selfassessment of daily habits to motor activities for health promotion. References

Janz KF, Lutuchy EM, Wenthe P, Levy SM (2008) Measuring activity in children and adolescents using self-report: PAQ-C and PAQ-A. Med Sci Sports Exerc 40(4):767-772

Michalopoulou M, Gourgoulis V, Kourtessis T, Kambas A, Dimitrou M, Gretziou H (2011). Step counts and body mass index among 9-14 years old Greek schoolchildren. J Sports Sci Med 10:215-221 Pangrazi RP, Beighle A, Sidman CL (2007) Pedometer power. Using pedometers in school and community. Human Kinetics, Champaign

\section{PE P}

\section{Analysis of the correlation between Cooper test and Léger test in a junior high school}

\author{
M. Tonietta ${ }^{1}$, G. Olla ${ }^{1}$, P. Moisè $\grave{e}^{1}$ \\ ${ }^{1}$ School of Exercise and Sport Sciences, University of Turin, Torino, \\ Italy; \\ ${ }^{2}$ Istituto Comprensivo Statale Buttigliera Alta-Rosta (TO), Italy
}

Purpose: Cooper and Léger tests are two of the most important indirect tests for the assessment of maximum aerobic power, $\mathrm{VO}_{2}$ max (Batista et al. 2017; Mackenzie 2015). The main purpose of this study is to offer teachers of physical education a comparison between the two tests to understand whether the methodological choice of one or the other in a school population is indifferent (Lovecchio et al. 2013).

Methods: A total of 188 students aged $13.02 \pm 0.85$ (108 males and 80 females). Their anthropometric characteristics can be summarized as follows: height $155 \pm 0.09 \mathrm{~cm}$, weight $47 \pm 12.45 \mathrm{~kg}$ and BMI $19.38 \pm 3.76 \mathrm{~kg} / \mathrm{m}^{2}$. After a short warm-up, the boys performed the two tests in the gym in two different sessions and subsequently the protocol of their $\mathrm{VO}_{2}$ max was calculated according to the results obtained in the tests.

Results: The data were processed using the Pearson correlation coefficient with significance established at 5\%. The analysis of the results shows that there is a significant correlation $(\mathrm{p}<0.001)$ between the two tests on the total of the sample; in the 108 males the correlation is $0.6808(\mathrm{p}<0.001)$, while in the females of 0.7347 $(p<0.001)$, that among the students with BMI $>25$ there is a correlation of $0.6686(\mathrm{p}<0.001)$, while in the group of pupils with 
BMI $<25$ the correlation is of 0.7185 ( $p<0.001)$, that in the students with height $>165 \mathrm{~cm}$ the correlation between the two tests is of 0.8306 ( $<<0.001$ ), while the group of pupils with height $<165 \mathrm{~cm}$ has a correlation of $0.7095(\mathrm{p}<0.001)$.

Conclusions: From the study emerges, confirming what is in the literature that the two tests can be overlapped in the school context, with subjects not in possession of a specific training. It does not seem that BMI and stature of the subjects influence the correlation.

As a result, both provide physical education teachers with a valuable tool to investigate the aerobic power of the school population.

References

Batista MB et al (2017) Rev Paul Pediatr 35(2):222-233

Lovecchio N et al (2013) Sport Sci Rev 22(3-4):217-228

Mackenzie B (2015) Green Star Media ISBN 9781910338516

\section{PE P}

\section{Fondazione Scoliosi Italia Onlus: a screening proposal in the primary and junior high school}

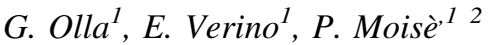 \\ ${ }^{1}$ School of Exercise and Sport Sciences, University of Turin, Torino, \\ Italy; \\ ${ }^{2}$ Istituto Comprensivo Statale Buttigliera Alta-Rosta (TO), Italy
}

Purpose: The start of screening for scoliosis began in 1963 in Aitken, a town with a population of about 10,000 inhabitants, in central Minnesota (Lonstein 1977). Many studies demonstrate the importance of school screening programs in the early detection of scoliosis or bad postural habits (Adobor et al. 2011, Deepak et al. 2017). Among the professionals who gravitate in the school environment, the teacher of physical and sporting sciences can play an important role in the observation of the posture of the students (Grivas et al. 2017). The Fondazione Scoliosi Italia Onlus, an association founded in 2009, has among its aims training teachers so that they can perform a 1 st level screening. The present study analyzes the data emerging from the screening among a group of students of a school in the province of Turin.

Methods: The sample group, consisting of 224 students (120 males, 104 females), aged $11.5 \pm 0.7$ height $152.0 \pm 7.0 \mathrm{~cm}$, weight $54.0 \pm 19.0 \mathrm{~kg}$ and BMI $23.2 \pm 6.0 \mathrm{~kg} / \mathrm{m}^{2}$, was observed using the protocol proposed by the F.S.I.O. During this screening, subjects were asked to wear a tight-fitting T-shirt and take off their shoes. The observation provided for the control on the frontal plane (anteriorposterior), on the sagittal plane in an upright position and with the Forward Bending Test.

Results: Of the total number of students examined, $45 \%$ were sent to medical examination ( $40 \%$ of females, $49 \%$ of males). In detail, $43 \%$ of the students at risk presented a more or less marked dorsal hump, while $19 \%$ had multiple problems. In females, the sum of these percentages (50-17\%) was higher than in males (37-20\%).

Conclusions: The collected data show how the high number of subjects with the presence of paramorphisms makes control activity at an early age extremely useful. The high number of families ( 90 out of 314 total, 30\%) who did not authorize screening, suggests a lack of sensitivity towards the usefulness.

References

Adobor RD et al (2011) Scoliosis 6:23

Deepak AS et al (2017) Malays Orthop J 11(1)

Grivas TB et al (2007) Scoliosis 2:17

Lonstein JE (1977) Clin Orthop Relat Res 33-42

\section{PE $P$}

\section{Analysis of Body Mass Index (B.M.I.) in a primary and middle school students}

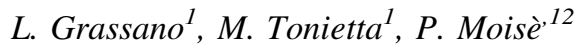 \\ ${ }^{1}$ School of Exercise and Sport Sciences, University of Turin, Torino, \\ Italy;
}

${ }^{2}$ Istituto Comprensivo Statale Buttigliera Alta-Rosta (TO), Italy

Purpose: Many researches show an increase in the percentage of overweight children in countries with an elevation (Doherty et al. 2017; Ezzati et al. 2017; Rao et al. 2016)

Being underweight, overweight, obese during childhood and adolescence (Aborea-Gomez et al. 2017; Ranjani et al. 2016).

The objective of the work is to investigate the characteristics of a sample of students of a school in the province of Turin.

Methods: A total of 281 subjects participated in the study (165 males and 116 females) height $1.55 \mathrm{~m} \pm 0.25 \mathrm{~m}$, weight $52 \mathrm{~kg} \pm 25 \mathrm{~kg}$ and BMI $22.1 \pm$ 7.9. During the class each stature and weight were measured with a statimeter and a scale. To define overweight and obesity, the BMI-for-age percentile growth charts are used to indicate the size and growth patterns of children and teens in the United States. Results: In girls the condition between 85-95th percentile is equal to $14 \%$ while over $95 \%$ is present with $7 \%$ of cases, with a total of $21 \%$ of subjects at risk. In boys, on the other hand, overweight is the same (14\%), but obesity increases compared to before with $14 \%$. Furthermore, the data show a substantial increase in male obesity with the increase in the age of the students. The decades are $11 \%$ while the fourteen are $16 \%$. Differently in the female gender the values are similar in the age groups, only 13 year olds have considerably higher values, with percentiles between $85-95$ equal to $26 \%$ and over $95-21 \%$. Conclusions: The results underline the opportunity to find solutions to cope with the phenomenon of weight gain, such as a more active quality of life and a balanced diet not only at home, but also in schools, places where children spend most of their time.

\section{References}

Ezzati M et al (2017) Lancet 390(10113):2627-2642

Doherty E et al (2017) Econ Hum Biol 27(PA):84-92

Rao DP et al (2016) Health Promot Chronic Dis Prev Canada 36(9)

Ranjani $H$ et al (2016) Indian J Med Res 143(2):160-174. https://doi.org/10.4103/0971-5916.180203 


\section{PE P}

\section{Influence of the Turkish Get $\mathrm{Up}$ in explosive power performance in a group of middle school students}

\author{
G. Olla ${ }^{1}$, D. Valiante $^{1}$, P. Moisè ${ }^{1} 2$ \\ ${ }^{1}$ School of Exercise and Sport Sciences, University of Turin, Torino, Italy; \\ ${ }^{2}$ Istituto Comprensivo Statale Buttigliera Alta-Rosta (TO), Italy
}

Purpose: The Turkish Get-Up (TGU), which can be defined as Functional exercises are often incorporated in training regimens to prevent injury and enhance performance. It combines features of a lunge, bridge, and side plank into a functional whole body exercise (Ayash et al. 2014; Liebenson et al. 2011).

The purpose of this study is to evaluate how much exercise can influence the ability to express explosive power.

Methods: A total of 42 students (22 males 20 females) aged $12.5 \pm 0.7$ height $154,5 \pm 9.1 \mathrm{~cm}$, weight $44.0 \pm 15.5 \mathrm{~kg}$ and BMI $18.1 \pm 14.3$ were divided in two groups, group A and group B. The group A, experimental group, composed of 21 students (11 males and 10 females) age $12.5 \pm 0.7$ height $157,5 \pm 13.4 \mathrm{~cm}$, weight $40.0 \pm 9.9 \mathrm{~kg}$ and BMI $15.9 \pm 1.2$. The group B, control group of 21 students (11 males and 10 females) height $157 \pm 5.6 \mathrm{~cm}$, weight $45.0 \pm 14.1 \mathrm{~kg}$ and BMI $18.0 \pm 4.4$. The experimental group performed a protocol of exercises aimed at learning the TGU executive technique for about 15 min twice a week during physical education lessons, the control group carried out the normal p.e. lessons. The explosive power was measured with the standing long jump.

Results: The analysis of the results was performed using the Student $t$ test for paired samples. It emerged that after 8 weeks of work the experimental group obtained an improvement of $4.1 \mathrm{~cm}(\mathrm{p}<0.001)$ while the group control showed an improvement of $0.4 \mathrm{~cm}$ (ns). If we separate, however, the experimental group in two subgroups (males and females) we discover that the group of males improves of $5.1 \mathrm{~cm}$ $(p<0.001)$ while the female group shows an improvement of $1.5 \mathrm{~cm}$, higher than that of the group control, but not significant.

Conclusions: The work seems to show that the practice of TGU influences the ability to express explosive power in a group of middle school students, but the improvement seems to be significant in the male sample and present but not significant in the female sample. A study of a larger sample could have more weight on the investigation. References

Ayash A et al (2012) Int J Athl Ther Train 17:8-13

Liebenson C et al (2011) J Bone Mov Ther 15(1):125-127

\section{PE P}

Field-based tests commonly used in children and adolescents practising sport to assess health-related components of physical fitness: an overview within the ESA programme

\author{
G. Tabacchi ${ }^{1,2}, F$. Neşe şahin ${ }^{3}$, M. Kizilyalli ${ }^{3}$, R. Genchi ${ }^{2}$, \\ M. Basile ${ }^{2}, M_{\text {. Kirka }}^{2}$, C. Silva ${ }^{4}$ N. Loureiro ${ }^{4}$, \\ Y. Demetriou ${ }^{5}$, D. Sturm ${ }^{5}$, S. Pajaujene ${ }^{6}$, I.J. Zuoziene , $^{6}$ \\ M. Gòmez-Lòpez ${ }^{7}$, G.F. Lòpez Sanchez ${ }^{7}$, A. Rada ${ }^{8}$,

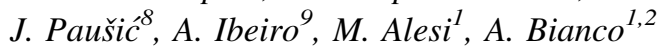

${ }^{1}$ Department of Psychological, Pedagogical and Educational Sciences, University of Palermo, Viale delle Scienze, Ed. 15, 90128; Palermo, Italy;

${ }^{2}$ Palermo University Sport Center (CUS Palermo), Via Altofonte, 80, 90129; Palermo, Italy;

${ }^{3}$ Department of Sport and Health, Faculty of Sport Sciences, Ankara University, Gölbaşi Yerleşkesi Spor Bilimleri Fakültesi 06830 Gölbaşi, Ankara, Turkey;

${ }^{4}$ Escola Superior de Desporto de Rio Maior (IPSantarém), Av. Dr. Mário Soares, 20413, RIO Maior, Portugal.

${ }^{5}$ School of Sport and Health Sciences, Technical University of Munich, Uptown Munich Campus D, Georg-Brauchle-Ring 60/62, 80992 Munich, Germany;

${ }^{6}$ Department of Coaching Science, Lithuanian Sports University, Sporto 6, Kaunas, LT-44221, Lithuania;

${ }^{7}$ Department of Physical Activity and Sport. Faculty of Sports Sciences, University of Murcia, Spain. Calle Argentina, 19, 30720 San Javier, Murcia, Spain;

${ }^{8}$ Faculty of Kinesiology, University of Split, Teslina 6, 21000; Split, Croatia;

${ }^{9}$ Escola Profissional de Aveiro, Rua Dr. Francisco Ferreira Neves. Apartado 567, Barrocas. 3800-510 Aveiro, Portugal

Purpose: The health-related components of physical fitness (PF) are considered important markers of current and future health in young and adult populations and are related to the ability of performing different sports. The aim of the present study is to provide an overview of the most used field-based tests to assess health-related PF components in children and adolescents practising sport.

Methods: A systematic review was conducted within the Enriched Sport Activities (ESA) Program, funded by the European Commission, to retrieve original articles that included the use of field-based tests in sportive young people. Papers published from 2007 in English/Spanish/Italian languages and targeted to healthy children/ adolescents were searched. For the purpose of this work, only the tests assessing health-related components of PF (muscular strength, muscular endurance, cardiovascular endurance and flexibility) were considered.

Results: Among the 1,133 items retrieved a total of 100 papers matched the inclusion criteria and were included in the final database. Muscular strength of lower and upper body was assessed in $66 \%$ of the studies, through a variety of jumps: vertical jumps $(62.1 \%)$ and standing broad jumps (48.5\%) were the most frequently used to assess lower body strength, while hand dynamometry $(12.1 \%)$ and medicine ball throw (10.6\%) for upper body; other upper body tests used that assess also muscular endurance were sit-ups $(25.7 \%)$ and push-ups (13.6\%). $23 \%$ of the studies assessed muscular endurance, mainly through multistage fitness tests (MSFT) (21.7\%) and line-drill tests $(13.0 \%)$; around half papers used sport-specific tests. Cardiovascular endurance was assessed in $54 \%$ of the papers, through mainly shuttle run intermittent recovery tests $(37.0 \%)$ such as Yo-yo $(24.1 \%)$. 
Flexibility was assessed in 19 studies, through sit and reach test in $84.2 \%$ of the papers.

Conclusions: High heterogeneity was evidenced between the used field-based tests. Some of them were demonstrated to be suitable for young populations and were used more frequently, so they were taken into greater consideration from the authors for the selection of a fitness battery to be used in the ESA project.

\section{PE $P$}

\section{The Daily Mile is feasible in Italy too: running or walking every day for 15 min improve the physical fitness of primary school children}

\author{
G. Boccia ${ }^{1}$, P. Moisè $\grave{e}^{2,3}$, C. Ruffa ${ }^{2}$,

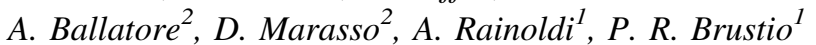 \\ ${ }^{1}$ NeuroMuscularFunction Research Group, School of Exercise \\ and Sport Sciences, Department of Medical Sciences, University \\ of Turin, Turin, Italy; \\ ${ }^{2}$ School of Exercise and Sport Sciences, SUISM, University of Turin, \\ Torino, Italy; \\ ${ }^{3}$ Istituto Comprensivo Statale Buttigliera Alta-Rosta, Scuola \\ Secondaria di primo grado "G. Jaquerio", Buttigliera Alta, Torino, \\ Italy
}

Purpose: The Daily Mile is a physical activity program growing in popularity internationally. Each day, during class time, children run or walk outside for $15 \mathrm{~min}$ (about 1 mile) at a self-selected pace. A recent study reported that, in Scotland among primary school children, the Daily Mile intervention is effective at increasing levels of physical activity, physical fitness and improving body composition. We aimed to explore the feasibility and effectiveness of the Daily Mile in Italian schools.

Methods: We conducted a quasi-experimental repeated measures pilot study in five primary schools in the neighborhood of Turin: after presenting the program to the teachers, the classes were divided in those who agreed to introduce the Daily mile (experimental group EXP, $N=540$ ) and those who did not intend to introduce the Daily Mile (control group $\mathrm{CON}, \mathrm{N}=339$ ). In the experimental group the Daily Mile was conducted for 3 months, while pupils at the control group followed their usual curriculum. Outcomes were 6-min run test, standing long jump, body mass index (BMI), and roundness index. Acceptability, satisfaction, perceived appropriateness and students' behavior were collected from the teachers using surveys and focus groups.

Results: After correction for age, significant improvements were observed in the experimental group relative to the control group for the 6-min run test $(\mathrm{EXP}+3.5 \%, \mathrm{CON}+0.9 \%$, ANOVA $(\mathrm{F}(1,478)=5.866, \mathrm{P}=0.035)$ and for standing long jump (EXP $+4.7 \%, \mathrm{CON}+2.8 \%$, ANOVA $(\mathrm{F}(1,788)=4.229, \mathrm{P}=0.040)$, but not for BMI and roundness index. Overall, teachers were satisfied with the project and found it suitable for their school context and easy to implement.

Conclusions: The intervention was easily and safely implemented, and it was considered suitable for the daily routine of Italian primary schools. The findings show that the Daily Mile intervention was effective at increasing physical fitness. These findings have relevance for Italian teachers and policymakers.

\section{Reference}

Chesham et al. The Daily Mile makes primary school children more active, less sedentary and improves their fitness and body composition: a quasi-experimental pilot study. BMC Med. 2018

\section{PE P \\ High University Education: functional training specialist}

\author{
D. Grassi ${ }^{1}$, C. Galbusera ${ }^{1}$, M. Neri ${ }^{2}$, M. Quarantelli ${ }^{l}$ \\ ${ }^{1}$ San Raffaele University Rome; \\ ${ }^{2}$ Federazione Italiana Fitness
}

Purpose: Typical exercises of functional training have a growing interest among both physical exercise practitioners, fitness and competitive sports disciplines. The increasing attention of practitioners regarding the methodological aspects of physical exercise suggests the opportunity to recall the physiological and mechanical bases of human movement by applying theoretical knowledge.

Methods: Due to the need of instructors to satisfy the increasing number of demanding practitioners, UniSanRaffaele and Italian Fitness Federation (FIF) have developed the first Advanced Training course oriented to FUNCTIONAL TRAINING. The purpose of the course was the application of the theoretical assumptions with reference to tools and exercise proposals typical of FUNCTIONAL TRAINING in order to obtain established and measurable training results. The educational offer was divided into 8 modules of $16 \mathrm{~h}$ each, including 4 modules in the gym, 3 modules in the classroom with university professors, 1 module in the evaluation class. The learning assessment and its consolidation were carried out through e-tivities, individual or cooperative exercises in the virtual classroom. The virtual classroom is based on MOODLE on the Telematic University website. It allows asynchronous interactions between students and tutors, who can develop exercises together, according to their time availability, reconciling their professional and personal needs.

Results: The students were involved for over $200 \mathrm{~h}$. For the final evaluation the students presented works created in a cooperative form, in which they developed training proposals aiming at achieving specific and individual performance goals, customized according to the initial evaluation of the practitioners.

Conclusions: Blended learning is an education program that combines online digital media, traditional classroom methods and exercise training sessions. A proper alternation of real and virtual classrooms and the integration of academic and training activities in a fitness contest allows a better development of professional skills.

\section{Reference}

Yagcioglu O (2011) Blended learning in higher education framework, principles and guidelines by D. Randy Garrison and Norman D. Vaughan—book review. Eur J Educ Stud. ISSN 25011111. 


\section{PE P}

\section{Evaluation of physical fitness in 11 years old students in Northern Italy: a powerful tool to improve physical education curricula}

\section{A. Cudicio, M. Cogliati, R. Perini, C. Orizio}

Department of Clinical and Experimental Sciences, University of Brescia, Italy

Purpose: This study was designed to evaluate the physical fitness (PF) in 11 years old students of 7 schools in Brescia. The aim was to provide functional data useful for motor activity promotion by physical education (PE) teaching in middle-lower school.

Methods: To assess the four main components of the PF we measured: (1) anthropometric data, i.e. height $(\mathrm{m})$, weight $(\mathrm{kg})$ to calculate BMI $\left(\mathrm{kg} / \mathrm{m}^{2}\right)$; (2) aerobic fitness through the six minutes walking test (6MWT); (3) muscular fitness through the standing broad jump test (SBJT); (4) joint flexibility from the V-Sit and reach test (VSR). Statistical analysis was performed by 2-way ANOVA.

Results: 349 children were tested, 257 Italian (I) and 74 Not-Italian (NI); I: 127 males (M) and 130 females (F); NI: $41 \mathrm{M}$ and $33 \mathrm{~F}$. NI children belonged to different ethnic groups.

\begin{tabular}{|c|c|c|c|c|c|c|c|c|}
\hline & \multicolumn{2}{|l|}{ I-M } & \multicolumn{2}{|l|}{$\mathrm{I}-\mathrm{F}$} & \multicolumn{2}{|l|}{ NI-M } & \multicolumn{2}{|l|}{ NI-F } \\
\hline & Mean & SD & Mean & SD & Mean & SD & Mean & SD \\
\hline BMI & 19.3 & 3.3 & 19.5 & 3.7 & 20.7 & 3.6 & 19.9 & 4.5 \\
\hline $6 \mathrm{MWT}$ & 720 & 59 & 697 & 54 & 704 & 75 & 683 & 52 \\
\hline SBJT & 154 & 22 & 144 & 21 & 145 & 24 & 137 & 21 \\
\hline VSR & -7 & 7 & 4 & 10 & -5 & 8 & -1 & 10 \\
\hline
\end{tabular}

Statistical differences between groups. $B M I$ I vs NI; M-I vs M-NI; $S B J T$ I vs NI; $\mathrm{M}(\mathrm{I}+\mathrm{NI})$ vs $\mathrm{F}(\mathrm{I}+\mathrm{NI}) ; \mathrm{M}-\mathrm{I}$ vs F-I; VSR $\mathrm{M}(\mathrm{I}+\mathrm{NI})$ vs $\mathrm{F}(\mathrm{I}+\mathrm{NI}) ; \mathrm{M}(\mathrm{I})$ vs F(I)

Conclusions: I group data. BMI mean value of both $\mathrm{M}$ and $\mathrm{F}$ is close to the 50th percentile according to a previous study concerning North of Italy children. Nonetheless, $15 \%$ of $\mathrm{M}$ and $11 \%$ of $\mathrm{F}$ have to be considered overweight [1]. 6MWT and SBJT mean results are even better than those reported for other age-matched European children $[2,3]$. VSR data are strongly influenced by gender. $M$ show marked negative results, while $\mathrm{F}$ show good flexibility. This difference should be considered in PE curriculum.

NI group data. Only two of the PF descriptors are different compared to I data, being BMI and SBJT value higher and lower, respectively, than those of I counterpart.

Generally, the resulting overall PF of the investigated students is in line with the literature data. The level of PF can be considered adequate, making this population able to follow PE curriculum aimed to include physical activity in daily life as a tool to discourage its abandon in adulthood.

References

Cacciari E et al (2002) Eur J Clin Nutr 56:171-180

Ulrich S et al (2013) BMC Pulm Med 13:49-60

Ortega FB et al (2011) Br J Sports Med 45:20-29
MOVEMENT LEARNING AND PSYCHOPHYSIOLOGICAL DEVELOPMENT

\author{
158 ML P \\ Adaptation to incompatible virtual surgeries \\ across multiple days
}

\author{
D. Borzelli ${ }^{1}$, S. Gurgone ${ }^{2}$, P. De Pasquale ${ }^{1}$, D.J. Berger ${ }^{3}$, \\ G. Acri' ${ }^{1}$ A. d'Avella ${ }^{1,3}$ \\ ${ }^{1}$ Department of Biomedical and Dental Sciences \\ and Morphofunctional Imaging, University of Messina, Italy \\ ${ }^{2}$ Department of Mathematical and Computer Sciences, Physical \\ Sciences and Earth Sciences, University of Messina, Italy; \\ ${ }^{3}$ Laboratory of Neuromotor Physiology, Santa Lucia Foundation, \\ Rome, Italy
}

Purpose: Myoelectric control of a virtual end-effector during multidirectional isometric force generation allows to investigate motor adaptation after a simulated perturbation of muscle pulling directions (virtual surgeries). When the perturbation is incompatible with the muscle synergies generating the force, subjects do not adapt, in accordance with the prediction that adaptation is hard because it requires learning of new muscle synergies. However, failure of learning may be due to the limited time available to practice. We have developed and tested the feasibility of a multi-day protocol to assess whether longer practice leads to better adaptation.

Methods: Subjects had their right arm, forearm, and shoulder fixed to a splint connected to a multi-axis force transducer. Electromyographic (EMG) activity was recorded from 13 shoulder and arm muscles during isometric force generation and muscle synergies were identified using non-negative matrix factorization. A monitor displayed in real-time the position of a cursor, controlled by the muscle activations according to a linear mapping of the recorded EMG. Subjects were instructed to displace the cursor to reach eight targets arranged in a circle on a horizontal plane. In each session, the subjects initially practiced with the linear mapping best fitting the generated force (baseline) and then with a perturbed mapping (incompatible virtual surgery) that did not allow to generate all planar force directions using the muscle synergies. Performance was quantified by the fraction of trials in which the cursor reached the target and by the angular error of the cursor initial movement direction.

Results: The linear mapping was robust across multiple days. Myoelectric control was also robust across days, as subjects were able to perform the task in baseline conditions across multiple days using the mapping estimated in the first day. Preliminary results indicated that subjects' performance improved across days but also showed differences across participants.

Conclusions: It is possible to use myoelectric control to characterize adaptation to an incompatible virtual surgery across multiple days. Further experiments will be required to better quantify learning rates and interindividual differences.

\section{Reference}

Berger, DJ, Gentner, R, Edmunds, T, Pai, DK, and d'Avella A (2013) Differences in adaptation rates after virtual surgeries provide direct evidence for modularity. J Neurosci 33(30):12384-12394 


\section{ML $P$}

Effects of kinesiotaping on motor performance in musicians suffering from focal hand dystonia: a pilot study

\section{R. Bravi ${ }^{1}$, E. J. Cohen ${ }^{1}$, C. I. Ioannou ${ }^{2}$, E. Altenmüller ${ }^{2}$, D. Minciacchi ${ }^{1}$}

${ }^{1}$ Department of Experimental and Clinical Medicine, University of Florence, Florence, Italy;

${ }^{2}$ Institute of Music Physiology and Musicians' Medicine, Hanover

University of Music, Drama and Media, Hanover, Germany

Purpose: Task-specific training and learning time are crucial factors to achieve and preserve high level of performance in a particular domain. Yet, repetitive use of a specific body segment for an extensive period can induce a deterioration of voluntary motor control due to maladaptive plastic changes in the sensorimotor system by interacting with other triggering factors such as psychological stresses, neuromuscular trauma, along with genetic susceptibility. In a musical setting, intensive training regimes can be associated with loss of motor control, leading to the manifestation of focal dystonia, also known as musician's dystonia (MD). MD is a movement disorder compromising the playing ability of musician and in many cases terminates musical careers. The study investigates the effects of Correction Kinesio Taping (CKT) intervention, compared to Sham Kinesio Taping (SKT), on fine motor control.

Methods: Seven affected musicians performed musical exercises designed to explore different motor patterns. A tailored CKT intervention on affected fingers was provided based on the dystonic pattern that each patient manifested while playing. Motor performance was video-documented and four independent experts assessed blindly the general performance and fingers' posture on visual analogue scales (VASs). In addition, self-report assessments of the movement abilities have also been assessed. Finally, muscular activity and co-activation index of wrist agonists and antagonist muscles were assessed.

Results: No different beneficial effect of CKT and SKT interventions was reported from the four experts on general performance and fingers' posture. Benefits offered by CKT were lost when the bandage was removed. Musicians estimated that CKT was ineffective in improving their general playing ability. In addition, no significant changes in co-activation of wrist antagonist muscles during and after interventions were observed.

Conclusions: These preliminary results suggest that CKT intervention may be useful in normalizing dystonic pattern and improving playing ability in selected musicians with FHD.

\section{References}

Krampe RT, Ericsson KA (1996) Maintaining excellence: deliberate practice and elite performance in young and older pianists. J Exp Psychol Gen 125:331-359

Altenmüller E, Jabusch HC (2009) Focal hand dystonia in musicians: phenomenology, etiology, and psychological trigger factors. J Hand Ther 22:144-155

Ioannou CI, Altenmüller E (2014) Psychological characteristics in musician's dystonia: a new diagnostic classification. Neuropsychologia 2014;61:80-88

Bravi R, Quarta E, Cohen EJ, Gottard A, Minciacchi D (2014) A little elastic for a better performance: kinesiotaping of the motor effector modulates neural mechanisms for rhythmic movements. Front Syst Neurosci 8:181

Bravi R, Cohen EJ, Quarta E, Martinelli A, Minciacchi D (2016) Effect of direction and tension of kinesio taping application on sensorimotor coordination. Int J Sports Med 37:909-914
160 ML $P$

When non-dominant is better than dominant: kinesiotape modulates asymmetries in timed performance during a synchronization-continuation task

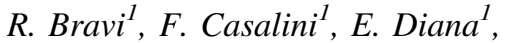 \\ E. J. Cohen ${ }^{1}$, A. Martinelli ${ }^{2}$, A. Gottard ${ }^{3}$, D. Minciacchi ${ }^{1}$ \\ ${ }^{I}$ Department of Experimental and Clinical Medicine, University \\ of Florence, Florence, Italy; \\ ${ }^{2}$ Department of Information Engineering, University of Florence, \\ Florence, Italy; \\ ${ }^{3}$ Department of Statistics, Informatics, Applications, University \\ of Florence, Florence, Italy
}

Purpose: There is a growing consensus regarding the specialization of the non-dominant limb (NDL)/hemisphere system to employ proprioceptive feedback when executing motor actions. In a wide variety of rhythmic tasks the dominant limb (DL) has advantages in speed and timing consistency over the NDL. Recently, we demonstrated that the application of Kinesio ${ }^{\circledR}$ Tex (KT) tape, an elastic therapeutic device used for treating athletic injuries, improves significantly the timing consistency of isochronous wrist's flexion-extensions (IWFEs) of the DL. We argued that the augmented precision of IWFEs is determined by a more efficient motor control during movements due to the extra-proprioceptive effect provided by KT. In this study, we tested the effect of KT on timing precision of IWFEs performed with the DL and the NDL, and we evaluated the efficacy of KT to counteract possible timing precision difference between limbs.

Methods: Young healthy subjects performed with and without KT (NKT) a synchronization-continuation task in which they first entrained IWFEs to paced auditory stimuli (synchronization phase), and subsequently continued to produce motor responses with the same temporal interval in the absence of the auditory stimulus (continuation phase). Two inter-onset intervals (IOIs) of 550-ms and 800-ms, one within and the other beyond the boundaries of the spontaneous motor tempo, were tested. Kinematics was recorded and temporal parameters were extracted and analyzed.

Results: Our results show that limb advantages in performing proficiently rhythmic movements are not side-locked but depend also on speed of movement. The application of KT significantly reduces the timing variability of IWFEs performed at 550-ms IOI. KT not only cancels the disadvantages of the NDL but also makes it even more precise than the DL without KT.

Conclusions: The superior sensitivity of the NDL to use the extrasensory information provided by KT is attributed to a greater competence of the NDL/hemisphere system to rely on sensory input. The findings in this study add a new piece of information to the context of motor timing literature. The performance asymmetries here demonstrated as preferred temporal environments could reflect limb differences in the choice of sensorimotor control strategies for the production of human movement.

\section{References}

Goble DJ, Brown SH (2007) Task-dependent asymmetries in the utilization of proprioceptive feedback for goal-directed movement. Exp Brain Res 180:693-704

Goble DJ, Brown SH (2010) Upper limb asymmetries in the perception of proprioceptively determined dynamic position sense. J Exp Psychol Hum Percept Perform 36:768-775

Bravi R, Quarta E, Cohen EJ, Gottard A, Minciacchi D (2014b) A little elastic for a better performance: kinesiotaping of the motor effector modulates neural mechanisms for rhythmic movements. Front Syst Neurosci 8:181 


\section{ML $P$}

Reaction time and dominance in elderly. Relationships with the motor task.

\author{
F. Campoli $^{1,3}$, M. Panzarino ${ }^{1,3}$, C. Romagnoli ${ }^{2}$,

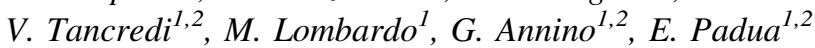 \\ ${ }^{1}$ Università Telematica San Raffaele, Roma, ItaliaDip. di \\ "Promozione delle scienze umane e della qualità della vita"; \\ ${ }^{2}$ Università di Roma Tor Vergata, Roma, Italia; \\ ${ }^{3}$ Accademia Nazionale di Cultura Sportiva
}

Purpose: This study aims to investigate the association between reaction time of the dominant and non-dominant hand in elderly individuals and to underline the gender differences.

Methods: 60 subjects $(68.4 \pm 4.4$ years $), 18$ men and 42 women, healthy, free from injury and without any physical problem that could affect the test results. The tests are the baseline reaction Times, the plate tapping test and foot tapping test.

Results: Descriptive statistical procedures are presented as mean and the percentage changes $(\Delta \%)$ were calculated for each tests and men/women differences. The significance level were performed of $\mathrm{p}<0.05$.

In baseline reaction time test, the non-dominant hand performs a lower reaction time than the dominant hand of about $41 \mathrm{~ms}$ $(p=0.01)$. Men have, in both hands, a better reaction time to stimulation than women over $50 \mathrm{~ms}(\mathrm{p}=0.008)$ but the difference between the non-dominant and the non-dominant hand is similar to both sexes and is about $12 \%(\mathrm{p}<0.05)$.

In the plate tapping test, on the other hand, the dominant hand is more reactive than the non-dominant hand $(7 \%, \mathrm{p}=0.001)$.

In the foot test, the difference between the dominant and nondominant feet is $2 \%(\mathrm{p}=0.01)$. Men records 1 touch more not statistically significant $(\mathrm{p}=0.1)$ and women it 0.4 taps $(\mathrm{p}=0.006)$. as the plate tapping test, men recorded 5 foot taps more than women $(\mathrm{p}=0.005)$.

Conclusions: Males have faster reaction times than females and the difference in performance between the tests performed could be due to the difficulty of the required motor task and the preferred hand is not always a good guide to the dominant hemisphere. Usually, a dominant (and faster) right hand implies a dominant left hemisphere. The side of the body with the longer reaction time (not always the side with the non dominant hand) is the side with the dominant hemisphere but if the task is more complex, as in the tapping test the reaction time difference between the left and right hands is remarkable contrary.

\section{References}

Jeeves MA, Moes P (1996) Interhemispheric transfer time differences related to aging and gender. Neuropsychologia 34(7):627-636

Rose SA, Feldman JF, Jankowski JJ, Caro DM (2002) A longitudinal study of visual expectation and reaction time in the first year of life. Child Dev 73(1):47
162 ML P

The central nervous system plasticity allows

the recovery of a lost motor function

from the homologous antagonist nerve

\author{
Papalia $^{1,2}$, E. Magaudda ${ }^{1}$,D. Buda ${ }^{2}$, N. De Salvo ${ }^{2}$, \\ A. Gennaro, L. Garufi ${ }^{2}$, S. Doddis ${ }^{2}$ \\ ${ }^{1}$ Department of Biomedical and Dental Sciences \\ and Morphofunctional Imaging, University of Messina, Italy; \\ ${ }^{2} \mathrm{MS}$ in Theory and Methods of Preventive and Adapted Physical \\ Activities, University of Messina
}

Purpose: The aim of the study is to assess brain plasticity through its ability to reprogram the function of a cortical motor area in that of its antagonist. Knowledge of the neurophysiological mechanisms underlying this phenomenon can clarify the mechanisms of development of certain coordinating abilities in normal or with motor deficits subjects.

Methods: In ten adult female rats, the left median nerve was repaired by end-to-side neurorrhaphy after epineuriotomy on the radial nerve at the middle of the brachium. Median nerve functional recovery was then assessed by the grasping test until week-30 postoperative. Before nerve withdrawal, the functional anatomy of median and radial nerves was assessed by electrical stimulation. Repaired nerves were then processed for resin embedding, and semi-thin sectioning were taken for nerve fiber histomorphometry by dissector method.

Results: Repaired median nerves were all repopulated by nerve fibers regenerating from the radial donor nerve and voluntary motor control of the flexor muscles innervated by the median nerve was progressively recovered.

Conclusions: Lost function of median nerve has effectively been recovered from pool of axons belonging from antagonistic radial nerve thanks to their corresponding cortical adaptation based on unfathomable brain plasticity mechanism. Further studies are necessary to understand if this occurrence is possible by other induction methods without surgical anatomical changes and, obviously, prudence should be taken in translating animal research to human application.

\section{References}

Lou L, Shou T, Li Z, Li W, Gu Y (2006) Transhemispheric functional reorganization of the motor cortex induced by the peripheral contralateral nerve transfer to the injured arm. Neuroscience 138:1225-1231

Lundborg G (2000) Brain plasticity and hand surgery: an overview. J Hand Surg 25B:242-252

Papalia I, Cardaci A, d'Alcontres FS, Lee JM, Tos P, Geuna S (2007) Selection of the donor nerve for end-to-side neurorrhaphy. J Neurosurg 107(2):378-382 


\section{MORPHOLOGICAL SCIENCES IN SPORT AND EXERCISES}

\section{MS P \\ Peroxisome proliferator-activated receptor $\gamma$ coactivator $1 \alpha$ expression levels in soleus and $E D L$ muscles after exercise}

\author{
F. Macaluso ${ }^{1,2,3}$, F. Scalia ${ }^{2}$, D. D'Amico ${ }^{2}$, F. Cappello $^{2,3}$, \\ F. Farina ${ }^{2}$, V. Di Felice ${ }^{2}$, R. Barone B $^{2,3}$

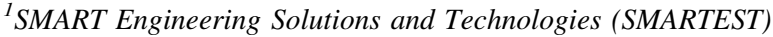 \\ Research Center, eCampus University, Italy; \\ ${ }^{2}$ Department of Experimental Biomedicine and Clinical \\ Neurosciences (BioNeC), University of Palermo, Italy; \\ ${ }^{3}$ Euro-Mediterranean Institute of Science and Technology (IEMEST), \\ Italy
}

Purpose: Peroxisome proliferator-activated receptor $\gamma$ coactivator $1 \alpha$ (PGC1 $\alpha$ ) is a transcriptional coactivator that controls the expression of gene involved in the regulation of fatty acid oxidation and glucose metabolism. PGC1 $\alpha$ is considered the "master regulator of mitochondria", as it regulates mitochondrial transcription factors. It has been reported that PGC1 $\alpha$ and its isoforms are involved in mitochondrial biogenesis, fibre type switching, stimulation of fatty acid oxidation, and resistance to muscle atrophy. Recently, we observed that endurance exercise increased the expression of PGC1 $\alpha 1, \alpha 2$, and $\alpha 3$ isoforms in murine soleus muscle.

Methods: In the present study we used thirty healthy male and female mice (BALB/c AnNHsd) divided in sedentary $(\mathrm{CN})$ and trained (TR) groups. TR mice ran for $60 \mathrm{~min}$ at a speed of $5.5 \mathrm{~m} / \mathrm{min}$ and were sacrificed after 30 and $240 \mathrm{~min}$ after the end of acute bout of endurance exercise (TR-30'-TR-240' respectively). CN mice did not perform any controlled physical activity. All mice were sacrificed by cervical dislocation and soleus and Extensor Digitorum Longus (EDL) muscles were dissected. Further, PGC1 $\alpha$ isoform expression levels were evaluated by qRT-PCR.

Results: The obtained results showed a significant increase in total PGC1 $\alpha$ isoform in response to acute exercise in the soleus and EDL muscles in TR-30' mice compared to all the other groups $(\mathrm{p}<0.05)$. Acute exercise induced significant increase of PGC1 $\alpha 1$ isoform gene expression levels in the soleus muscle of TR30' male and female mice $(\mathrm{p}<0.05)$ while a significant decrease was observed in $E D L$ muscle $(\mathrm{p}<0.05)$. Moreover, PGC1 isoform $\alpha 2$ and $\alpha 3$ gene expression increased in male and female TR $30^{\prime}$ groups only in EDL muscle $(\mathrm{p}<0.05)$. We did not observed any change soleus muscle. Furthermore, PGC1 isoform $\alpha 4$ gene expression level was not detected in any muscle samples.

Conclusion: These preliminary results, showing the increased expression levels of the isoform $\alpha 2$ and $\alpha 3$ only in $E D L$ muscle immediately after acute exercise, should represent a very interesting and innovative data that might open new ways in the study of the role of these proteins in the skeletal muscle adaptation during exercise. Acknowledgments: This work was supported by grants from MIUR PRIN-2012.

Reference

Barone et al (2016) Skeletal muscle Heat shock protein 60 increases after endurance training and induces peroxisome proliferator-activated receptor gamma coactivator $1 \propto 1$ expression. Sci Rep 27:6
164 MS P

Myotendinous junction plasticity: tenocyte activation is modulated by exercise

\section{Curzi, D. Burini, E. Falcieri}

Department of Biomolecular Sciences, University of Urbino Carlo Bo, 61029, Urbino, Italy

Purpose: Myotendinous junction (MTJ) is the key structure of locomotor system through which contractile force can be transmitted from muscle fibers to tendon tissue. The plasticity of MTJ has been demonstrated in different physiological conditions. In previous works we revealed the MTJ ability to reduce or to increase its structural complexity and the contact surface between tissues in relationship to muscle atrophy and exercise protocols, respectively [1, 2]. The molecular mechanisms and the different stages of these morphofunctional adaptations are still unknown and the aim of this work is to identify which tissue component could play a pivotal role in modulating this anatomical interface.

Methods: By means of transmission electron microscopy observations, an interesting high presence of tenocytes close to the MTJ has been revealed.

Results: At high magnification, these tenocytes display an increased amount of rough endoplasmic reticulum in the exercised group, compared to sedentary one. The exercise ability to increase tenocyte rough endoplasmic reticulum amount has already been demonstrated and correlated to the production of collagen fibers and extracellular matrix components [3].

Conclusions: Morphological observations of tenocyte behavior close to MTJ reveal their activation following a resistive exercise protocol, suggesting a key role of the cells in the modulation of MTJ morphology and plasticity.

\section{References}

1. Curzi D et al (2013) Growth hormone plus resistance exercise attenuate structural changes in rat myotendinous junctions resulting from chronic unloading. Eur J Histochem 57:247-254

2. Curzi D et al. (2014) Morphological adaptation and protein modulation of myotendinous junction following moderate aerobic training. Histol Histopathol 30:465-472

3. Salamanna F et al. (2014) Metabolic and cytoprotective effects of in vivo peri-patellar hyaluronic acid injections in cultured tenocytes. Connect Tissue Res 56:35-43 


\section{BIOMOLECULAR AND NUTRITIONAL ASPECTS OF EXERCISE AND SPORT}

\section{BN P}

\section{The impact of fitness level and aerobic exercise on acute and chronic stress response of Immune cells}

\author{
E. Grazioli ${ }^{1}$, C. Fantini ${ }^{\text {, }}$, I. Dimauro ${ }^{I}$, A. Antonioni ${ }^{1}$, \\ F. Guidotti ${ }^{l}$, P. Sgrò ${ }^{l}$, L. Di Luigi ${ }^{l}$, L. Capranica ${ }^{l}$, \\ D. Caporossi ${ }^{1}$
}

${ }^{I}$ Department of Movement, Human and Health Sciences, University of Rome "Foro Italico", Italy

Purpose: The heats shock proteins (HSPs) are exercise-induced stress-response molecules that facilitate the cellular remodelling processes. HSP response to exercise, as physiological stressor, is well characterized in rodents, while in humans the type, intensity, duration of exercise and the timing of response are less insightful. The first aim of this study is to analyse the acute effect of a single bout high intensity aerobic exercise on HSPs and antioxidant level from lymphocytes of trained and untrained young adults males. The second goal is to evaluate the adaptation of biomarkers' response after 4 days of high intensity aerobic training in untrained subjects.

Methods: After approval by the Ethical Committee (RIF.CE: 4521) 17 healthy male subjects $(25 \pm 6$ years old) were enrolled in the study and divided in two group: the Athlete Group (AG, 11 subjects) enrolled in the acute aerobic protocol, and the Untrained Group (UG, 7 subjects), who were analysed under the acute aerobic protocol and then enrolled in the 4 days aerobic training. Blood samples were collected before, $3 \mathrm{~h}$ and $24 \mathrm{~h}$ after the acute exercise. Further sampling was occurred at the end of 4th day and $24 \mathrm{~h}$ after aerobic training. PBMCs were isolated from whole blood and immediately frozen. Whole proteins were extracted and analysed for HSPs (HSP70, HSP27, p-HSP27, $\alpha \mathrm{B}$-crystallin, $\mathrm{p}-\alpha \mathrm{B}$-crystallin), signaling molecules (p38MAPK) and antioxidants (Catalase, MnSOD, TrxR1) content.

Results: Our data confirm a late ( $24 \mathrm{~h})$ exercise-induction in PBMCs of HSP70 in all subjects.

Contrary to some published data, we were unable to verify any expression of $\alpha \mathrm{B}$-crystallin, in PBMCs, neither at basal or after exercise-induced stress, whereas HSP27 seems to show a positive modulation both in expression and in activation, being untrained subjects more responsive to exercise. This study promises to improve not only our knowledge on the systemic response to a specific acute aerobic exercise but also to verify the role of stressresponse proteins in the systemic adaptation to aerobic training in sedentary subjects in order to unravel, at least in part, the molecular mechanism linked to the health effect of this specific exercise regime.

\section{References}

1. Henstridge DC, Febbraio MA (2016) Hargreaves M3. Heat shock proteins and exercise adaptations. Our knowledge thus far and the road still ahead. J Appl Physiol 120(6):683-691. https://doi.org/10.1152/japplphysiol.00811.2015

2. Morton JP, Kayani AC, McArdle A, Drust B (2009) The exercise-induced stress response of skeletal muscle, with specific emphasis on humans. Sports Med 39(8):643-662. https://doi.org/10.2165/00007256-200939080-00003.

\section{BN P \\ Comparison of exogenous ketone bodies chronic supplementation and ketogenic diet on some metabolic parameters}

\author{
N. Sponsiello ${ }^{1}$, D. Campaci $^{2}$
}

${ }^{1} M D$, Board ESNS;

${ }^{2}$ Biologist, Teacher NonSoloFitness SNC

Purpose: Ketogenic diet is often used in sport nutrition with the scope to induce a fast weight reduction and an increase in fat utilization.

The purpose of the study is to understand if a chronic ketone bodies salts supplementation, associated to a balanced diet, could mime the effect of the ketogenic diet on the fat metabolism and weight loss. Methods: 10 subjects were divided in two groups. One group has been administrated, for 15 days, a ketogenic diet (KD) and the other one a balanced diet supplemented with exogenous ketone bodies (SP). Before starting and at the end of this period Respiratory Ratio (indirect calorimetry) and the Body composition (BIVA analysis) were evaluated.

Results: KD group reduced significantly Respiratory Ratio $(-0.0636 ; p=0,015)$, body weight $(-4.2 \mathrm{~kg} ; \mathrm{p}=0.05)$ and fat mass $(-3.6 \mathrm{~kg} ; \mathrm{p}=0.041)$. SP group did not change any of the evaluated parameters.

Conclusions: First of all is necessary to emphasise that exogenous ketone body salts assumption increases ketonemia for a short time after ingestion (an average of $2 \mathrm{~h}$ and half with a dose of $11.8 \mathrm{~g}$ of $\beta$ $\mathrm{OHB})$. This time could be shorter to induce any significantly chronic effect. From our data, 15 days of chronic exogenous ketone bodies supplementation cannot substitute ketogenic diet when it's prescribed with the objective to obtain fast weight reduction or to induce a major fat utilization, for example during a fat loss program or during the preparation of an endurance athlete.

\section{References}

Cox PJ et al (2016) Nutritional ketosis alters fuel preference and thereby endurance performance in athletes. Cell Metab 24:256-268 Evans et al (2017) Metabolism of ketone bodies during exercise and training: physiological basis for exogenous supplementation. J Physiol 595:2857-2871

Newport MT et al (2015) A new way to produce hyperketonemia: use of ketone ester in a case of Alzheimer's disease. Alzheimer's Dementia 11:99-103

Paoli A (2014) Ketogenic diet for obesity: friend or foe? Int J Environ Res Public Health 11:2092-2107

Paoli A et al (2015) Effects of twenty days of the ketogenic diet on metabolic and respiratory parameters in healthy subjects. Lung 193:939-945 


\section{BN P}

\section{Effects of football training on TNF-alpha mediated inhibition of human LHCN-M2 myoblasts differentiation}

\author{
A. Alfieri ${ }^{1,2}$, D. Vitucci $^{3}$, A. Mancini $^{1,2}$, S. Orrül $^{1,2,3}$, \\ P. Buono ${ }^{1,2,3}$
}

${ }^{I}$ Department of Movement Sciences and Wellness, University Parthenope, Naples, Italy;

${ }^{2}$ CEINGE-Biotecnologie avanzate, Napoli, Italy; ${ }^{3}$ IRCCS SDN Foundation, Naples, Italy

Purpose: TNF-alpha (TNF-a), an inflammatory cytokine, is poorly expressed in skeletal muscle. This cytokine plays a pivotal role in muscular atrophy by inhibiting myogenic differentiation [1]. We recently analyzed the effects of different training on myogenic differentiation in LHCN-M2 human myoblast cells [2].

Here we investigated the effects of soccer training in counteracting the myogenic differentiation inhibition in human myoblast LHCN-M2 cells mediated by TNF-a.

Methods: LHCN-M2 cells were treated with $20 \mathrm{ng} / \mathrm{ml}$ of TNF-a for $4 \mathrm{~d}$ in Differentiation Medium (DM) supplemented with $0.5 \%$ serum pool from n.4 male subjects soccer trained $(\mathrm{SO} \geq 180 \mathrm{~min} / \mathrm{w})$ or from untrained subjects (HC); pre-treatment with IGF-1 was also performed, in some cases. Myotube formation, pAkt and myosin beta proteins expression were determined in order to evaluate myogenic differentiation.

Results: LHCN-M2 myotube formation was inhibited after 4 days of TNF-a $(20 \mathrm{ng} / \mathrm{ml})$ treatment and a reduction of pAKT and myosin beta protein expression was observed. IGF-1pre-treatment partially restored pAkt levels, allowing cells to differentiate in the presence of TNF-a; SO serum treatment partially rescued the TNF-a inhibition of myogenic differentiation in LHCN-M2 cells.

Conclusions: Our preliminary results suggest that TNF-a inhibits the myogenic differentiation of LHCN-M2 cells and this inhibition occurs at least in part via IGF-1. Soccer training partially rescued the inhibition on myogenic differentiation induced by TNF-a, suggesting an attractive therapeutic role in counteracting muscular atrophy in ageing.

References

Zhao Q et al (2015)

Vitucci D. et al (2018)

Acknowledgements: This work was supported by "Bando per la Ricerca competitiva, triennio 2016-2018 quota C" grant from the University of Naples "Parthenope"

\section{BN P \\ Effects of a ketogenic diet on triathlon's athletes performance: a pilot trial}

\section{A. Paoli, F. Zambolin, D. Grigoletto, G. Marcolin \\ Department of Biomedical Sciences, University of Padova, Italy}

Purpose: Sport nutrition guidelines support the idea that carbohydrates $(\mathrm{CHO})$ are fundamental for performance, mainly for endurance. However recently published studies on long distance athletes suggested some advantages of a low $\mathrm{CHO}$, high fat approach, i.e. ketogenic diets $[1,2]$. We aimed to investigate the effects of ketogenic diet $(\mathrm{KD})$ on triathlon athletes.
Methods: Sixteen expert athletes (age $30.67 \pm 10.46$ years) were enrolled in the pilot trial. Eight athletes followed a ketogenic diet (KD) for 5 weeks whilst other eight followed a standard western diet (WD). Subjects were tested for maximal oxygen consumption $\left(\mathrm{VO}_{2} \mathrm{max}\right)$, peak power output (PPO), respiratory ratio (RR), heart rate, $\mathrm{VO}_{2}$, and blood lactate during one hour cycloergometer test at $45 \%$ of PPO, and body composition through electrical impedance (BIA). A two way ANOVA treatment $\mathrm{x}$ time test was performed.

Results: After 5 weeks subjects in the KD group showed a significant increase of $\mathrm{VO}_{2} \max \left(\mathrm{ml} \mathrm{O}_{2} \times \mathrm{kg}\right.$ of body weight $\times$ min $)$ $35.68 \pm 3.86$ to $36.6 \pm 5.1 ;$ a significant increase of PPO $(140 \pm 5.29$ to $145 \pm 9$ ), a significant decrease of fat mass (from $10.28 \pm 3.04$ to $7.86 \pm 2.53 \mathrm{~kg}$ ), lactate (from $2.02 \pm 0.7$ to $1.2 \pm 0.6$ ) and of RR (from $0.86 \pm 0.03$ to $0.79 \pm 0.01$ ). No changes of lean body mass and time to exhaustion were detected.

Conclusions: Our data suggest that a ketogenic diet performed for a sufficient time ( $>4$ weeks) could improve some parameters related to performance in high level triathlon athletes.

\section{References}

Cox PJ, Kirk T, Ashmore T et al (2016) Nutritional ketosis alters fuel preference and thereby endurance performance in athletes. Cell Metab 24(2):256-268

Volek JS, Freidenreich DJ, Saenz C et al (2016) Metabolic characteristics of keto-adapted ultra-endurance runners. Metabolism 65(3):100-110

\section{BN P}

\section{Relationship between food literacy and gross motor and pre-literacy skills in pre-schoolers from the Training-to-Health Project}
G. Tabacchi ${ }^{1}$, G. Battaglia ${ }^{1,2,3}$, M. Alesi $^{1}$, A. Palma $^{1,2,3}$, M. Bellafiore $e^{1,2,3}$

${ }^{1}$ Department of Psychological, Pedagogical and Educational Sciences, University of Palermo, Sicily, Italy;

${ }^{2}$ Sport and Exercise Sciences Research Unit, University of Palermo, Sicily, Italy;

${ }^{3}$ Regional Sports School of CONI Sicilia, Sicily, Italy;

${ }^{4}$ Department of Sciences for Health Promotion and Mother Child Care "G. D'Alessandro"

Purpose: Food literacy is a recent concept encompassing food knowledge and skills, and is related with every aspects of life including health, culture and environment. The assessment and development of food knowledge and skills is important since the early childhood. The aim of this study is to assess the relationships between food literacy and gross motor and pre-literacy skills in a sample of pre-schoolers.

Methods: The sample was recruited within the Training-to Health Project conducted in the Palermo City Council. A total of 928 children aged 3-5 years from all 21 municipal kindergartens were recruited. Brief education sessions on nutritional topics and practical activities were performed by the children, and a validated 4 domainstoolkit with prearranged sheets was compiled to evaluate a food literacy score. Gross motor and pre-literacy skills were assessed by the Italian version of gross motor development test and the PRCR-2/ 2009. Bivariate and multivariate regression analyses were performed to estimate associations, with significance level set at $\mathrm{p}<0.05$.

Results: The adjusted coefficients between food literacy and gross motor abilities were significant for the quotient of gross motor development (QGMD) and in particular for the component of 
locomotor skills (coef $0.03, \mathrm{p}<0.01$; coef $0.16, \mathrm{p}<0.001$, respectively). Almost all the correlations with the pre-literacy skills (lower time and fewer errors in recognising letters and naming objects) were statistically significant (coefficients ranged from -0.02 to 0.47 , $\mathrm{p}<0.001)$.

Conclusions: The present study adds new information to the literature regarding food literacy in pre-schoolers and suggests that increased food literacy can be found in children with better QGMD, locomotor and pre-literacy skills. Information provided by the present study emphasizes the need of assessing the food knowledge and skills and developing locomotor, pre-reading and pre-writing skills since preschool age to enhance success probabilities at school.

\section{BN P \\ Influence of obesity on balance and physical fitness in women}

\section{Innocenti ${ }^{1}$, G.P. Emerenziani ${ }^{1}$, M.C. Gallotta ${ }^{1}$, L. Guidetti ${ }^{l}$, C. Baldari ${ }^{1,2}$}

${ }^{1}$ Department of Movement, Human and Health Sciences, University of Rome "Foro Italico", Rome, Italy;

${ }^{2}$ eCampus University, Novedrate (CO), Italy

Purpose: The terms overweight and obesity are defined as abnormal/ excessive fat accumulation with Body Mass Index (BMI) cutoffs values of $25 \mathrm{~kg} / \mathrm{m}^{2}$ and $30 \mathrm{~kg} / \mathrm{m}^{2}$, respectively. The World Health Organization (WHO) estimates that more than 1.9 billion adults are overweight worldwide; with 600 million obese adults in 2014. In the US, the situation is exacerbated with 78.6 million adults being classified as obese in 2012.

The aim of the present study was to investigate the influence of obesity on static balance in women.

Methods: Forty-four participants, 23 obese women (group OB: BMI $>30 \mathrm{~kg} / \mathrm{m}^{2}$ ) and 21 normal-weights non-sportive women (group CONT: BMI between 18.5 to $24.9 \mathrm{~kg} / \mathrm{m}^{2}$ ) were recruited.

The characteristics of the CONT and OB group were respectively: body mass $37.5 \pm 15.9$, age $45.2 \pm 13.2$ years, BMI $21.2 \pm 1.6$ and $38.1 \pm 4.4 \mathrm{~kg} / \mathrm{m}^{2}, 24.5 \pm 5.2$ and $46.7 \pm 5.0 \%$ Fat-max.

The body mass composition was assessed with a Body Impedance Assessment (TANITA InnerSuan). To evaluate the level of physical activity was given the Six-minute walking test (6MWT) with an OMNI-scale of perception of fatigue (RPE). To assess the static balance, the One-leg standing balance (OLSB) test was used, this test was performed in four different conditions: open eyes (OLSBo), closed eyes (OLSBc), with also mathematic cognition task (OLSBoc and OLSBcc).

Results: Independent $t$ tests showed significant differences between group OB and group CONT for BMI ( $p<0.002)$, weight $(\mathrm{p}<0.001)$, but not in FFM ( $>$ > 0.005).

Independent $t$ tests showed very significant differences between CONT and OB group: in OLSBo (eyes open) $(\mathrm{p}<0.001)$, in OLSB c (eyes open with mathematical task) $(\mathrm{p}<0.002)$, in OLSBc (eyes closed) $(\mathrm{p}<0.001)$, in OLSBcc (eyes closed with mathematical task) $(\mathrm{p}<0.001)$; significant differences were found in the independent $t$ test test on the SMWT, between OB and CONT groups: in SMWTdist (distance traveled) $(\mathrm{p}<0.001)$, in SMWTvel (velocity traveled) $(\mathrm{p}<0.001)$ and in SMWT-RPE (Perceived Exertion scale) $(\mathrm{p}<0.001)$.

Conclusion: It can be affirmed the hypothesis that there is indeed a significant difference, between static balance capacity between obese and normal-weight subjects. We propose in future studies to investigate: (1) if there are the same differences by male gender; (2) how physical activity affects (SMWT) the ability to stay in static balance.

\section{References}

Meng H, O'Connor DP, Lee B-C, Layne CS, Gorniak SL (2016) Effects of adiposity on postural control and cognition. Gait Posture 43:31-37

Incidence of bone loss falls, and fractures after Roux-en-Y gastric bypass for morbid obesity (2009). Appl Nurs Res 22:35-41

\section{BN P}

\section{Effects of combined exercise and recreational soccer Small Sided Games program on the expression of adiponectin in type 2 diabetes subjects.}

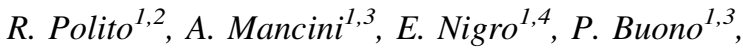 \\ A. Daniele D,2 $^{1,2}$
}

${ }^{l}$ CEINGE-Biotecnologie Avanzate S.c.a r.l., Naples, Italy; ${ }^{2}$ Dipartimento di Scienze e Tecnologie Ambientali Biologiche Farmaceutiche, Università della Campania "Luigi Vanvitelli Caserta, Italy;

${ }^{3}$ Dipartimento di Scienze Motorie e del Benessere, Università "Parthenope" Naples, Italy;

${ }^{4}$ Dipartimento di Scienze Cardio-Toraciche e Respiratorie, Università degli Studi della Campania, "Luigi Vanvitelli", Via Leonardo Bianchi c/o Ospedale Monaldi, 80131 Naples, Italy

Purpose: Adiponectin has multivalent beneficial biological functions in the regulation of energy homeostasis, insulin sensitivity, and in inflammation processes. Adiponectin circulates as LMW, MMW, HMW oligomers, the latter being the most biologically active. Serum adiponectin levels are decreased in obese individuals and patients with type 2 diabetes (DM2) [1]. We evaluated whether a combined exercise, Indoor Muscular Activation (IMA) and recreational soccer Small Sided Games (SSG) program, affects body composition and serum adiponectin levels and its oligomeric status.

Methods: 6 sedentary subjects (40-63 years) affected by DM2 were enrolled at the Diabetes Unit, University of Naples "Federico II". They participated in an exercise program constituted by Indoor Muscular Activation (IMA) [twice weekly/6 weeks, resistance $(2 \times 15$ at $50-60 \% 1 \mathrm{RM})$ /aerobic training (30 min at 65\% FCmax)] followed by 12 weeks of SSGs in a pitch $25 \times 40 \mathrm{~m}$. Games consisted of three 12-min bouts, interspersed by $2 \mathrm{~min}$ of recovery, once a week. Body composition (BIA), total adiponectin levels (ELISA) and oligomeric status (Western Blot) were determined at T0 (baseline), T1 (6 weeks), T2 (12 weeks of SSGs) for all participants.

Results: After 6 weeks of IMA and 18 weeks of IMA plus SSGs, we found: a reduction in \% Fat Mass [21.8 (T2) vs. 23.8 (T0)]; an increase in Free Fat Mass [62.9 (T2) vs. 60.9 (T0) kg] $(p<0.05)$; and improvement in \% Total Body Water [56.0 (T2) vs. 54.0 (T0)] and in $\%$ Intracellular Water [58.6 (T2) vs. 57.6 (T0)]. In addition, a statistically significant increase of serum adiponectin levels $(13.2 \mu \mathrm{g} / \mathrm{ml}$ (T1), $13.7 \mu \mathrm{g} / \mathrm{ml}$ (T2) vs. $11.86 \mu \mathrm{g} / \mathrm{ml}$ (T0), $p<0.05$ and $p<0.005$, respectively) was found. Furthermore, we observed, in each patient, a better adiponectin profile with an increase in HMW oligomers.

Conclusions: Our results suggest that the combined IMA and SSGs physical exercise training statistically improves not only body composition but it is sufficient to substantially increase total adiponectin and to ameliorate adiponectin oligomeric status in DM2.

Acknowledgements: This work was supported by University of Naples Parthenope, "Bando per la ricerca individuale, annualità 
2016" and "Bando per la Ricerca competitiva, triennio 2016-2018 quota C"

Reference

De Rosa et al (2013) Eur J Endocrinol 37:43

\section{BN P}

\section{Identification of candidate biomarkers related to healthy longevity in muscles from lifelong football players: an integrative-omics approach}

\author{
E. Imperlini ${ }^{1}$, A. Mandola ${ }^{2,3}$, A. Mancini ${ }^{2,3}$, A. Alfieri ${ }^{2,3}$, \\ D. Vitucci ${ }^{1}$, M. Caterino ${ }^{4}$, M.B. Randers ${ }^{5}$, J.F. Schmidt ${ }^{5}$, \\ M. Hagman ${ }^{5}$, T. Rostgaard ${ }^{5}$, P. Krustrup ${ }^{5}$, M. Ruoppolo ${ }^{4}$, \\ P. Buono ${ }^{1,2}$, S. Orrù̀ ${ }^{1,2}$ \\ ${ }^{1}$ IRCCS SDN Foundation, Naples, Italy; \\ ${ }^{2}$ Department of Movement Sciences and Wellness, University \\ Parthenope, Naples, Italy; \\ ${ }^{3}$ CEINGE-Biotecnologie avanzate, Napoli, Italy; \\ ${ }^{4}$ Department of Molecular Medicine and Medical Biotechnologies, \\ University Federico II, Naples, Italy; \\ ${ }^{5}$ Copenhagen Centre for Team Sport and Health, Department \\ of Nutrition, Exercise and Sports, University of Copenhagen, \\ Copenhagen, Denmark,.
}

Purpose: Football training is associated with higher $\mathrm{VO}_{2} \max$ and improved cardio-respiratory fitness. Increasing evidence shows the beneficial effects of football training on cardiovascular, metabolic and musculoskeletal health variables [1]. However, few studies had analyzed the molecular effects of lifelong football training on muscle metabolism in healthy subjects [2, 3]. In this context, we have recently analyzed both the gene and protein expression profiles in muscle biopsies from Veteran Soccer Players (VSPs) compared to healthy age-matched untrained subjects (control group, CG). Here, we performed a combined analysis of transcriptomic/proteomic/metabolomic data to increase the confidence of findings and to maximize the coverage of the molecular signatures of healthy longevity related to football training.

Methods: The whole set of data were obtained from gene/protein/ metabolite expression profiling analyses (by using cDNA microarray, label-free proteomics and targeted metabolomics, respectively), in muscle biopsies from the same 12 male volunteers (65-75 years), 6 belonging to VSP and 6 to CG. Combined transcriptomic/proteomic/ metabolomic data were analyzed by bioinformatic tools to classify the identified differentially expressed genes/proteins and miRNA-targets according to gene ontology (GO) terms and to unravel relevant molecular networks.

Results: During the discovery phase for healthy longevity-related candidate biomarkers, our comprehensive data analysis demonstrated both the uniqueness and complementarities of the integrative-omics approach.

Conclusions: The integration of transcriptomics, proteomics and metabolomics is a useful tool to better unravel the molecular mechanisms underlying the healthy longevity effects of lifelong football training.

Acknowledgements: This work was supported by "Bando per la ricerca per il fondo di finanziamento individuale (annualità 2016 e 2017) e competitivo di gruppo (annualità 2016)" funded by University Parthenope, Naples, Italy.

References

Bangsbo J, Hansen PR, Dvorak J and Krustrup P (2015) Br J Sports Med 49:568-576
Alfieri A, Martone D, Randers MB, Labruna G, Mancini A, Nielsen JJ, Bangsbo J, Krustrup P, Buono P (2015) Mol Cell Probes 29:43-47 Mancini A, Vitucci D, Labruna G, Imperlini E, Randers MB, Schmidt JF, Hagman M, Andersen TR, Russo R, Orrù S, Krustrup P, Salvatore F, Buono P (2017) Eur J Appl Physiol 117:721-730

\section{BN P Implementation of omega 3 fats in young athletes}

\author{
R. Tudisco ${ }^{1}$, D. Gastaldi ${ }^{l}$, G. Abbate Daga ${ }^{1}$, D. Mozzone ${ }^{2}$,
} C. Tarozzo $O^{3}$, P. Brignolo ${ }^{4}, F$. Scarzella ${ }^{4}$

${ }^{1}$ Università degli Studi di Torino, Dipartimento di Scienze degli alimenti e della Nutrizione Umana;

${ }^{2}$ Istituto di Medicina dello Sport di Torino;

${ }^{3}$ Università degli Studi di Messina, Scuola di Specializzazione in Medicina dello Sport e dell'Esercizo Fisico;

${ }^{4}$ Università degli Studi di Palermo, Scuola di Specializzazione in Medicina dello Sport e dell'Esercizo Fisico

Purpose: Omega- 3 fats play an important role in the prevention of various diseases, especially cardiovascular ones. There are also evidences that Omega- 3 fats have a relationship with diseases related to the emotional and psychological sphere, such as anxiety and depression, and with some parameters characterizing physical performance, such as strength, aerobic endurance and muscle fatigue.

They are essential elements, in fact they are not produced by our body and must therefore be taken through the diet.

Studies have almost always been conducted on untrained healthy volunteers, on the elderly or on pathological subjects, while only few studies took into consideration a sport population. The aim of our study is to correlate some aspects of athletic performance with omega-3 fats levels.

Methods: Our study examined 27 male subjects between the ages of 18 and 25 ( 7 acted as a control) in a team of football elite and 2 basketball teams.

The subjects are analyzed at time zero and after 30 days.

At the beginning and at the end of the study they have carried out a blood chemistry test to evaluate the levels of omega-3 fats and a series of functional and psychological evaluations.

Results: After the first battery of evaluations, omega- 3 supplementation began in the intervention group. At the end of the 30 days they carried out blood tests and functional and psychological evaluations, in order to observe the blood chemistry changes and any differences in the various tests.

Conclusion: The implementation with omega- 3 improves body composition (fat mass, weight and circumferences), increases dynamic and isometric strength, fatigue tolerance during physical exercise and the psychological aspect referred to anxiety, mood and body perception.

\section{References}

Nelson DL, Cox MM (2014) I principi di biochimica di Lehninger (VI edizione), Zanichelli, pp 369-392, 684-705, 851-861

Doublier SM (2017) Corso di Laurea Magistrale in "Scienze degli Alimenti e della Nutrizione Umana-modulo di "Biochimica della Nutrizione". Capitolo 7-lipidi slide 9:35, 40 (1998) "Antropometria"-Enciclopedia Italiana di Scienze, Lettere ed Arti (Enciclopedia Treccani), Stampato

Spielberger CD, Gorsuch RL, Lushene R, Vagg PR, Jacobs GA (1983) Manual for the State-Trait Anxiety Inventory. Consulting Psychologists Press, Palo Alto 
Bertolotti G, Bettinardi O, Biondo R, Gucciardi M, Michielin P, Salvini A, Sanavio E, Turchi GP, Vidotto G, Zotti AM. Manualetto per l'uso preliminare della batteria CBA Sport

Mickleborough TD (2013) Omega-3 polyunsaturated fatty acids in physical performance optimization. Int $\mathbf{J}$ Sport Nutr Exerc Metab 23(1):83-96

Corder K, Newsham K, McDaniel JL, Ezekiel U, Weiss EP (2016) Effects of short-term docosahexaenoic acid supplementation on markers of inflammation after eccentric strength exercise in women. J Sports Sci Med 15(1):176-183

Jooyoung K, Joohyung L (2014) A review of nutritional intervention on delayed onset muscle soreness. Part I. J Exerc Rehabil 10(6):349-356

Lembke P, Capodice J, Hebert K, Swenson T (2014) Influence of Omega-3 (N3) Index on performance and wellbeing in young adults after heavy eccentric exercise. J Sports Sci Med 13(1):151-156

Jouris KB, McDaniel JL, Weiss EP (2011) The effect of omega-3 fatty acid supplementation on the inflammatory response to eccentric strength exercise. J Sports Sci Med 10(3):432-438

Mickleborough TD, Sinex JA, Platt D, Chapman RF, Hirt M (2015) The effects PCSO-524 ${ }^{\circledR}$, a patented marine oil lipid and omega-3 PUFA blend derived from the New Zealand green lipped mussel (Perna canaliculus), on indirect markers of muscle damage and inflammation after muscle damaging exercise in untrained men: a randomized, placebo controlled trial. J Int Soc Sports Nutr 12:10

Tinsley GM, Gann JJ, Huber SR, Andre TL, La Bounty PM, Bowden RG, Gordon PM, Grandjean PW (2016) Effects of fish oil supplementation on postresistance exercise muscle soreness. J Diet Suppl 21:1-12

Seung-Lyul O, Sang-Rok L, Jeong-Su K (2017) Effects of conjugated linoleic acid/n-3 and resistance training on muscle quality and expression of atrophy-related ubiquitin ligases in middle-aged mice with high-fat dietinduced obesity. J Exerc Nutr Biochem 21(3):11-18 Lee SR, Khamouim AV, Jo E, Zourdos MC, Panton LB, Ormsbee MJ, Kim JS (2017) Effect of conjugated linoleic acids and omega-3 fatty acids with or without resistance training on muscle mass in high-fat diet-fed middle-aged mice. Exp Physiol 102(11):1500-1512

Da Boit M, Sibson R, Sivasubramaniam S, Meakin JR, Greig CA, Aspden RM, Thies F, Jeromson S, Hamilton DL, Speakman JR, Hambly C, Mangoni AA, Preston T, Gray SR (2017) Sex differences in the effect of fish-oil supplementation on the adaptive response to resistance exercise training in older people: a randomized controlled trial 1,2. Am J Clin Nutr 105(1):151-158

Gordon IS (2016) The effects of dietary omega-3 s on muscle composition and quality in older adults. Curr Nutr Rep 5(2):99-105 Tsuchiya Y, Yanagimoto K, Nakazato K, Hayamizu K, Ochi E (2016) Eicosapentaenoic and docosahexaenoic acids-rich fish oil supplementation attenuates strength loss and limited joint range of motion after eccentric contractions: a randomized, double-blind, placebo-controlled, parallel-group trial. Eur J Appl Physiol 116(6), 1179-1188

Bhullar AS, Putman CT, Mazurak VC (2016) Potential role of omega3 fatty acids on the myogenic program of satellite cells. Nutr Metab Insights 9:1-10

\section{SPORT BIOMECHANICS AND TECHNOLOGY}

\section{SB P}

Kinematic analysis of the kip (artistic gymnastics)

\author{
A. Coser $^{1}$, N. Carazzai ${ }^{1}$, S. Maffesanti ${ }^{1}$, N. Dal Santo ${ }^{1}$, \\ M. Maggio $^{2}$, P. Zamparo ${ }^{1}$
}

${ }^{I}$ Department of Neuroscience, Biomedicine and Movement Sciences, University of Verona, Italy;

${ }^{2}$ Fondazione Bentegodi, sezione ginnastica artistica, Verona, Italy

Purpose: In artistic gymnastics, the kip is a basic skill on the women's uneven bars. This skill is used as both a mount skill and a connecting skill in a bar routine. This sequence of movements allows the gymnast to swing below the bar to arrive in a front support on the bar. The purpose of this study was to analyze the biomechanical characteristics of the kip performed by novice $(n=13 ; 10 \pm 1$ years of age; $3 \pm 1$ years of experience) and expert $(n=6,20 \pm 5$ years of age; $11 \pm 4$ years of experience) gymnasts.

Methods: All subjects performed a 10-min warm-up session (stretching of the upper and lower limb muscles) before data collection. A high speed camera (Canon Lumix F28, $200 \mathrm{fps}$ ) was utilized for kinematic analysis. Ten body landmarks (tip foot, heel, malleolus, knee, hip, shoulder, elbow, wrist, hand, head) were digitized by means of a free motion analysis software (https://physlets.org/traker/). The body centre of mass (BCoM) was calculated by using the Dempster data provided by Winter (2005); the time course of the BCoM kinetic (calculated based on both vertical and horizontal components), potential and total energies $(\mathrm{Et}=\mathrm{Ek}$ è Ep) during the kip were then computed and analysed; these data were then normalized by the subject's body mass (are thus expressed in $\mathrm{J} \mathrm{kg}^{-1}$ ).

Results: During the forward swing Ek and Ep change in opposition of phase (as in a pendulum) and Et decreases of about $20 \%$ compared to the starting position (with no differences between groups). In the backward swing expert gymnasts are able to increase their energy level (Et) more than novices $(39 \pm 4 \%$ vs. $25.7 \pm 7 \%$, p < 0.001$)$. This (positive) difference in Et corresponds to 6.2 and $4.7 \mathrm{Jkg}^{-1}$ of mechanical work provided by the muscles. No differences were observed between groups for maximal hip flexion (about $43^{\circ}$, during the backward swing) and extension (about $170^{\circ}$, during the forward swing) but the shoulder angle at the beginning of the backward swing was smaller in expert than in novices $\left(139^{\circ} \pm 8^{\circ}\right.$ vs. $152^{\circ} \pm 10^{\circ}$, $\mathrm{p}<0.001$ ).

Conclusions: Data reported in this study indicate that expert gymnasts are better able to raise their BCoM over the bar (larger Et and smaller shoulder angle), optimizing the effect of the swinging movement (the sequence of lengthening and shortening of the hip flexors).

\section{References}

Hay JG (1993) The biomechanics of sports techniques. Prentice Hall, $\mathrm{NJ}$

Winter DA (2005) Biomechanics and motor control of human movement. Wiley, NJ 


\section{SB P}

\section{Effects of electro-stimulation during resistance training on maximum strength levels: a 4-week pilot study}

\author{
S. Dell'Anna ${ }^{I}$, F. Nieddu ${ }^{I}$, L. Correale ${ }^{1}$, E. Codrons ${ }^{1}$, \\ E. Ricagno ${ }^{1}$, G.D. Jonghi Lavarini ${ }^{1}$, G. Liberali ${ }^{1}$, \\ C.A. Naldini ${ }^{1}$, M. Vandoni ${ }^{1}$ \\ ${ }^{1}$ Laboratory of Adapted Motor Activity (LAMA), Department \\ of Public Health, Experimental Medicine and Forensic Science, \\ University of Pavia, Pavia, Italy
}

Purpose: Electro-stimulation (EMS) is a technique used to produce a physiological muscular contraction similar to voluntary contraction. Several studies demonstrated positive effects of EMS in strength development. Resistance training (RT) is the standard protocol to enhance strength. It's not clear if EMS may be useful in association with resistance training to increase strength. The purpose of this study is to evaluate the effects of EMS in training for maximum strength on Smith Machine Back Squat (BS) and Barbell Bench Press (BP).

Methods: Twenty healthy participants $(12 \mathrm{M}, 8 \mathrm{~F})$ without RT experience were assigned to an experimental group (E) or a control group $(\mathrm{C})$. Both groups practiced a 4 -week (2 times/week) resistance training protocol preceded by 2 -week of familiarization, in addition group E wore and EMS device (Miha Bodytech) during each session. Baseline physical activity levels were calculated using IPAQ questionnaire. RT protocol consisted of 7 sets (3-5 repetition, $80-90 \%$ of $1 \mathrm{RM}$, rest between sets was $3 \mathrm{~min}$ ) for each session of training for the entire protocol. Before and after RT protocol each subject performed an indirect one maximum repetition (1RM) test for both BP and BS. A time (pre, post) $\mathrm{x}$ group $(\mathrm{E}, \mathrm{C})$ two-way repeated measures ANOVA was used $(\mathrm{p}<.05)$.

Results: Physical activity levels were similar in both groups. EMS group showed significant improvements in BS $(+12.4 \% ; \mathrm{p}<.05)$ and $\mathrm{BP}(+10.09 \% ; \mathrm{p}<.05)$ compared to pre testing. The same effect was observed in C group, BS $(+9.4 \% ; \mathrm{p}<.05)$ and BP $(+8.29 \%$; $\mathrm{p}<.05)$. No significant difference was found between group $\mathrm{E}$ and $\mathrm{C}$. Conclusions: We verified similar improvements in both groups $(\mathrm{E}$ and C) for maximum strength. Although we cannot affirm that EMS gives superior advantage instead of traditional training, we observed better enhancement in maximum strength in group $\mathrm{E}$.

\section{SB P}

\section{Cervical spinal kinematic analysis in young rowers at rowing ergometer during different frequency}

\author{
V. Giustino ${ }^{1,2}$, D. Zangla ${ }^{3,4}$, G. Messina ${ }^{1,2}$, K. Feka ${ }^{5}$, \\ F. Fischetti ${ }^{6}$, A. Iovane ${ }^{1}$, A. Palma ${ }^{3,7}$ \\ ${ }^{1}$ Department of Psychological, Pedagogical and Educational \\ Sciences, Posturology and Biomechanics Laboratory Research Unit, \\ University of Palermo, Palermo, Italy; \\ ${ }^{2}$ Posturalab Italia Research Institute, Palermo, Italy; \\ ${ }^{3}$ Department of Psychological, Pedagogical and Educational \\ Sciences, Sport and Exercise Sciences Research Unit, University \\ of Palermo, Palermo, Italy; \\ ${ }^{4}$ Italian Rowing Federation (FIC), Rome, Italy; \\ ${ }^{5}$ University of Palermo, Palermo, Italy; \\ ${ }^{6}$ Department of Basic Medical Sciences, Neuroscience and Sense \\ Organs, University of Bari, Bari, Italy; \\ ${ }^{7}$ Regional Sports School of CONI Sicilia, Sicily, Italy
}

Purpose: Several authors have been investigating the lumbar spinal kinematic that represents the most frequent injured anatomical region in rowers. While, only few studies have examined the cervical column. Furthermore, it is known that, during a competition, compensatory movements may affect negatively performance. The aim of this study was to evaluate if there are any differences in flexion (F) and extension (E) of the head movements in rowers during two different frequency tests.

Methods: Twelve young rowers $($ male $=5$; female $=7$; age: $13 \pm$ 0.85 years; height: $156.42 \pm 8.53 \mathrm{~cm}$; weight: $51.37 \pm 11.17 \mathrm{~kg}$ ), with 2-3 years of rowing experience, were enrolled in the study. F and $\mathrm{E}$ head movements were recorded while subjects were performing a sequence of 10 strokes at the frequency of 20 strokes/min $\left(\mathrm{T}_{0}\right)$ and during a separate sequence of 10 strokes at the frequency of 30 strokes/ min $\left(T_{1}\right)$ using rowing ergometer (Concept $2{ }^{\circledR}$ Mod. C; Indoor Rowing Srl; Morlupo, Roma, Italia). Cervical Range of Motion (ROM) on the sagittal plane was measured with the Moover ${ }^{\circledR}$ accelerometer and the freeStep ${ }^{\circledR}$ related software (Sensor Medica ${ }^{\circledR}$; Guidonia Montecelio, Roma, Italia). Parameters were considered as: the average flexion (AvF), the average extension (Av-E) and the average total angle of flexionextension (Tot-FE). Statistical analyses were performed using Statistica Software 12 (StatSoft ${ }^{\circledR}$, TIBCO $^{\circledR}$ Software Inc, Palo Alto, CA, USA). A paired $t$ test was used to compare differences between $\mathrm{T}_{0}$ and $\mathrm{T}_{1}$. The $p$ value was considered to be statistically significant at $p<0.05$.

Results: Comparison between $\mathrm{T}_{0}$ to $\mathrm{T}_{1}$ showed no significant difference for all considered parameters. However, we found an increase of the amplitude angle in Av-E movement $(+22.28 \%)$ and Tot-FE $(+10.47 \%)$, while in the other hand, a decrease of the Av-F movement $(-12.18 \%)$. According to technical indications of the Italian Rowing Federation, changes greater/less than $25 \%$ on $\mathrm{F}$ and $\mathrm{E}$ head movements, changing the frequency, may affect negatively the performance. When calculating the percentage between participants, only 2 of them showed a greater ROM $(-25$ to $+25 \%)$.

Conclusions: Our results indicated that young rowers showed changes on F-E ROM in relationship with higher stroke frequency and this may influence sports performance. Further studies are needed in order to clarify the relationship between head movements and performance, as well as to examine the implications of cervical spine injuries.

\section{References}

Bernardes F, Mendes-Castro A, Ramos J, Costa O (2015) Musculoskeletal injuries in competitive rowers. Acta Med Port 28(4):427-434

Leo Ng, Campbell A, Burnett A, Smith A, O’Sullivan P (2015) Spinal kinematics of adolescent male rowers with back pain in comparison 
with matched controls during ergometer rowing. J Appl Biomech 31(6):459-468

\section{SB P}

\section{Correlation study between gait parameters and cognitive status in unrestricted people with relapsing-remitting multiple sclerosis}

\author{
R. Minino ${ }^{1}$, M. Liparoti $^{1}$, M. Della Corte ${ }^{2,3}$, R. Rucco ${ }^{1}$, \\ M. Sparaco ${ }^{2,3}$, R. Capuano $^{2}$, P. Sorrentino ${ }^{4}$, \\ G. Sorrentino ${ }^{1,3}$, S. Bonavita ${ }^{2}$
}

${ }^{1}$ Department of Motor Sciences and Wellness, University of Naples "Parthenope", via Medina 40, 80133, Naples;

${ }^{2}$ Department of Medical, Surgical, Neurological, Metabolic and Aging Sciences MRI Research Center SUN-FISM, University of Campania "Luigi Vanvitelli", P.zza Miraglia 2, 80138, Naples; ${ }^{3}$ Hermitage Capodimonte Hospital, via Cupa delle Tozzole 2, 80131, Naples;

${ }^{4}$ Department of Engineering, University of Naples "Parthenope", Centro Direzionale Isola C4, 80133, Naples

Purpose: Aims of study were to detect, by 3D-GA, changes of gait pattern during usual walking (single task, SinT), motor and cognitive dual tasks (MotDT, CogDT) in pwRR-MS compared to HCs and to analyze the correlations between cognitive status scores and gait parameters.

Methods: 22 pwRR-MS and 21 healthy controls (HCs), underwent to cognitive and gait analysis [1] evaluations.

Results: During SinT pwRR-MS compared to HCs, showed an impairment of velocity [cycle time $(\mathrm{p}<0.05)$ ], stability [stance time $(\mathrm{p}<0.05)$, swing time $(\mathrm{p}<0.05)$ and swing time-CV $(\mathrm{p}<0.05)]$ and kinematic (increase of ankle dorsi-flexion $(\mathrm{p}<0.05)$ during mid and terminal stance phases of gait cycle) parameters. The CogDT and MotDT produced a slight impairment of spatiotemporal parameters as compared to SinT, indeed an increase of cycle time $(p<0.01)$, swing time $(\mathrm{p}<0.01)$ and double limb support $(\mathrm{p}<0.05)$ for CogDT were recorded. MotDT also caused an increase of thigh flexion $(\mathrm{p}<0.05)$ during the initial contact, loading response, mid swing and terminal swing phases of gait cycle. We also found an inverse correlation between the Stroop score and both cycle time $(\mathrm{p}<0.05)$ and swing time $(\mathrm{p}<0.05)$ during SinT. Another inverse correlations between the Stroop score and swing time $(\mathrm{p}<0.05)$ and verbal fluency score and double limb support-CV during MotDT were observed.

Conclusions: The study shows that 3D-Gait Analysis is a useful tool to verify slight motor impairment in pwRR-MS and suggests a role of cognition in the motor impairment in pwRR-MS.

Reference

1. Rucco R, Agosti V, Jacini F et al (2017) Spatio-temporal and kinematic gait analysis in patients with Frontotemporal dementia and Alzheimer's disease through 3D motion capture. Gait Posture $52: 312-317$
EXERCISE AND SPORT PSYCHOLOGY

\author{
178 SP P \\ "Improve your steps": a social-based intervention \\ to fight physical inactivity
}

\author{
F. Gallè ${ }^{1}$, D. Caso $^{2}$, V. Carfora ${ }^{3}$, A. Miele $^{1}$, \\ N. Postiglione $^{1}$, R. Ricchiuti ${ }^{l}$, G. Liguori $^{l}$ \\ ${ }^{1}$ Università di Napoli Parthenope; \\ ${ }^{2}$ Università di Napoli Federico II; \\ ${ }^{3}$ Università Cattolica del Sacro Cuore, Milano
}

Purpose: Today, physical inactivity is one of the main risk factors for health: in Europe, it causes more than 1 million deaths annually. In Italy, the $38.1 \%$ of the population is inactive; in Campania region, it accounts for $54.2 \%$ of people. The walking promotion may represent an effective prevention tool against this phenomenon.

During the month of May, 2018, the Universities Parthenope and Federico II of Naples started a pilot study of walking promotion which involved undergraduates of Movement Sciences and Psychology. The study is part of the initiative "Improve your steps", aimed at evaluating the efficacy of a motivational support provided via social media to promote the adoption of an active lifestyle.

Methods: All the students invited received recommendations about the importance of achieving at least 7.000 steps per day. At the start of the intervention, the International Physical Activity Questionnaire (IPAQ) and a questionnaire based on the "Theory of planned behavior" were administered online to the 153 students $(42.5 \% \mathrm{M}$, mean age 21 years) who accepted to take part to the study in order to evaluate their initial levels of daily physical activity and the psychological variables influencing their intention to walk. All the participants underwent the Rockport test to evaluate their aerobic fitness. These measurements will be repeated at the end of the study. The students enrolled were randomly distributed among four groups: - 2 intervention groups, whose participants receive through Facebook Messenger daily motivational messages regarding respectively physical (group $\mathrm{A}, \mathrm{n}=39$ subjects) and psychological (group B, $\mathrm{n}=39$ subjects) benefits of walking, who were invited to send the number of steps done per day counted through smartphone app;

-2 control groups ( $\mathrm{A}, \mathrm{n}=40$ and $\mathrm{B}, \mathrm{n}=35$ ) which don't receive daily motivational messages; only group A was invited to send the daily report of steps done.

Results and conclusions: The study will be completed at the end of June. The preliminary results show a good participation to the study and testify the regular daily achievement of the objective in both the intervention groups.

The final physical evaluations might confirm these observations.

\section{References}

1. Physical activity strategy for the WHO European Region 2016-2025. Copenhagen: WHO Regional Office for Europe, 2015

2. ISTAT. Indagine multiscopo "Aspetti della vita quotidiana", anno 2017

3. Ajzen I (1991) The theory of planned behavior. Org Behav Hum Decis Process 50(2):179-211

Keywords: Motivational intervention, Social media, Walking 


\section{SP P}

Homophobia in sports: analysis of a sample of students of sports and health science

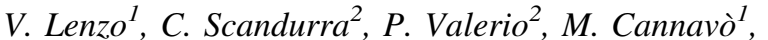 \\ A. Capocasale ${ }^{1}$, S. Riposo ${ }^{1}$, M. C. Scarfò ${ }^{1}, M$. Vadalà ${ }^{1}$, \\ M. C. Quattropani ${ }^{1}$
}

${ }^{1}$ Università degli Studi di Messina, Dipartimento di Medicina Clinica e Sperimentale;

${ }^{2}$ Università degli Studi di Napoli Federico II

Purpose: Sport environment can be defined as the competitive environment per excellence. The goal of most of athletes is to win, and not to partecipate. Several studies pointed out the presence of a set of stereotypes and heteronormative rooted values, which generate very evident homophobic behavior (Amodeo et al. 2017). The aim of this study is to evaluate the presence of a possible correlation between sport practice, homophobia, genderism and transphobia in a sample of students of Sports and Health Science, by assessing the different attitudes between these students and students of other classes.

Methods: The sample of partecipants consists of students of Sports science and students from other courses degree from University of Messina. Students filled a demographic questionnaire, a sexism scale, Homophobia Scale (HS), Genderism and Transphobia Scale (GTS) and TEIQue-SF, for trait-emotional intelligence.

Results: The results have shown the presence of a statistically significant correlation between the variables investigated.

References: Amodeo et al (2017) Homophobia and transphobia in a sample of Movement Sciences students: Implications for physical education teachers and coaches. La camera blu, 17, Sports contexts and gender perspectives

Keywords: Homophobia, Genderism, Transphobia, Sports and health science

\section{SP P}

\section{Cognitive functioning and sports practices}

\section{Lenzo ${ }^{1}$, L. Magaudda ${ }^{2}$, M. Cannavò ${ }^{1}$, A. Capocasale ${ }^{1}$, S. Riposo ${ }^{1}$, M. C. Scarfò ${ }^{l}$, M. Vadalà ${ }^{l}$, M. C. Quattropani ${ }^{l}$}

${ }^{1}$ Department of Clinical and Experimental Medicine, University Hospital "G. Martino".

${ }^{2}$ Department of Biomedical, Dental Sciences and Morphological and Functional Images, Postgraduate School of Sports Medicine.

University of Messina, Messina, Italy.

Purpose: Sport psychology helps the development of the athlete by enhancing his own skills, the knowledge and the overcoming of his own limits. A good management of anxiety and distress seems to be related to the performance, a good approach to workout, a good communication with the trainer and with his own body. The aim of this study was to assess the cognitive features in subjects involved in sport practicing, such as metacognition, motivation and anxiety.

Methods: The group consists of 50 subjects $(\mathrm{M}=25.86, \mathrm{SD}=5.87)$. They filled STAI X-1, STAI X-2, MCQ-30 e BREQ.

Results: Descriptive statistics has been used to better understand the cognitive factors implied during the athletic training and performance.
Conclusions: The knowledge of psychological factors implied in sport practicing allow a better understanding of the performance, and a better planning of it.

\section{References}

Nascinbene F (2002) Prospettive in psicologie dello sport, I.S.U Università Cattolica Milano

Keywords: Metacognition, motivation, anxiety, sports practices.

\section{SP P}

\section{A meta-analysis about the effects of stereotype threat of women's performances in sports and physical activity}

\section{A. Gentile ${ }^{1}$, S. Boca ${ }^{2}$}

${ }^{1}$ Ph.D. Program in Health Promotion and Cognitive Sciences, University of Palermo, Palermo, Italy;

${ }^{2}$ Department of Psychological, Pedagogical and Educational Sciences, University of Palermo, Palermo, Italy

Purpose: When an individual from a social group perform a task in which that particular group is "known to be weak", his/her performance could be undermined. This effect, known as "stereotype threat", has been studied for more than two decades within the psychological literature (Steele 1997; Steele and Aronson 1995). Many studies observed stereotype threat in different domains and related to different social groups (e.g., females and mathematics, elders and memory, African Americans and intelligence tasks) and several metaanalyses have been published about these studies. However, no metaanalysis about the effects of stereotype threat on women's performances in sport and physical activities had been conducted before.

Method: The current poster examines, with a meta-analytic technique, the effect sizes of 24 studies $(\mathrm{k}=24)$, published between 2006 and 2017. The average effect size was obtainedfollowing the Hedges and Olkin's approach (1985), based on Glass' method (1976), which suggest to calculate Cohen's $d$ with standardized mean difference. The potential publication bias was assessed through the funnel plot drawing and the Egger's regression test (Egger et al. 1997). Since the studies showed internal heterogeneity, it was necessary to hypothesize a set of potential moderators for explaining this variability.

Results: The current present meta-analysis found an effect of $d=.33$ (95\% CI .03; .63; $\mathrm{p}<.05)$ of 24 studies $(\mathrm{k}=24)$ published between 2006 and 2017. Both funnel plot and Egger's regression test revealed that no publication bias affected the publications. The effect of stereotype threat seems to be higher within sports and physical activities perceived as masculine.

Conclusions: Stereotype threat undermines female's performances and its effect is higher for sport and physical activities with masculine features.

Keywords: Gender stereotypes, Underperformance, Literature review, Sensorimotor activities

\section{References}

Egger M, Davey Smith G, Schneider M, Minder C (1997) Bias in meta-analysis detected by a simple, graphical test. BMJ 315:629-634 Glass GV (1976) Primary, secondary, and meta-analysis of research. Educ Res 5:3-8

Hedges LV, Olkin I (1985) Estimation of a single effect size: parametric and nonparametric methods, Chapter 5. In: Statistical methods for meta-analysis. Academic Press, San Diego, pp 75-106 Steele CM (1997) A threat in the air: How stereotypes shape intellectual identity and performance. Am Psychol 52:613 Steele CM, Aronson J (1995) Stereotype threat and the intellectual test performance of African Americans 
182 SP P

Orienteering as a mean to improve psycho-physical performance in subjects with autism spectrum disorder

\author{
F. Furnari ${ }^{1}$, L. Donato, G. Morsicato ${ }^{1}$ \\ ${ }^{1}$ Master's Degree Program in Sciences and Techniques of Preventive \\ and Adapted Motor Activities, University of Messina, Italy
}

Purpose: This study was aimed to evaluate the impact of the activities related to Orienteering on children and adolescents in a developmental phase and affected by autism, to correlate in a specific way the effects of this sport on some aspects of this condition, such as empathy, autonomy, ability to exercise, emotional and physical control as well as selection of decision-making strategies.

Methods: We have conducted a series of evaluations and measurements on the performances of subjects affected by autism during sessions aimed at propaedeutic activities and Orienteering contests. To this end, control groups have been established, together with internal groups of facilitators-observers (tutors).

Results: We have recorded a positive difference in the study group versus the control group, in relation to initial expectations, in decision-making capabilities; we have also recorded difficulties in changing point of view and impediments in the overall vision and perception of space, as well as in the interpretation of reality.

Conclusions: We believe that already in this starting phase, the research on the relationship between Orienteering and the strengthening of some deficit areas of subjects affected by autism (empathy, autonomy, ability to exercise emotional and physical control as well as selection of decision-making strategies) could facilitate a positive outcome in the traditional rehabilitation treatments, with significant improvements in the areas of personal autonomies.

\section{References}

Berthoz A (2011a) Fondements cognitifs de la perception de l'espace. In J.-F. Augoyard (Ed.), Faire une ambiance = creating an atmosphere : actes du colloque international Grenoble 10-12 septembre 2008. Grenoble, pp 121-132

Lobben AK (2004) Tasks, strategies, and cognitive processes associated with navigational map reading: a review perspective. Prof Geogr 56(2):270-281

McNeill C, Cory-Wright J, Renfrew T (1998) Teaching orienteering. Human Kinetics, Champaign

Di Tore PA (2016) Spatial navigation cognitive strategies, perspective taking and special educational needs: re-thinking orienteering sport in complexity. J Phys Educ Sport 16:2
POSTURAL APPROACH TO SPORT AND EXERCISE

\author{
183 PO P \\ Postural sway alterations linked to an high intensity \\ interval training session
}

\author{
A.R. Calavalle, D. Sisti, M. Gervasi, S. Amatori P. Sestili, \\ M.B.L. Rocchi
}

Department of Biomolecular Sciences, University of Urbino "Carlo Bo", Italy

Purpose: Balance, both dynamic and static, is crucial in many sports; on the other hand, both acute and chronic fatigue obviously plays an important role in the performance of athletes. The association between acute fatigue and the consequent postural strategies is not sufficiently known. Aim of this work was to highlight association between HIIT session and postural sway features.

Methods: Postural sway alteration, quantified using of Centre of pressure displacements during time (COPs) linked to high intensity interval training (HIIT) was investigated in a sample of 32 students $($ age $=21 \pm 1.5 ; \quad$ height $=1.69 \pm 0.10 \quad \mathrm{BMI}=22 \pm 2)$ of the University of Urbino. COPs were measured before HIIT test and $5 \mathrm{~min}, 1,24$ and $24 \mathrm{~h}$ later. The balance conditions were assessed by asking the subjects to stand in a bipedal postural configuration, barefoot upright on a force platform as immobile as possible for $60 \mathrm{~s}$. Using Time domain measures: mean distance from centre of sway (mDIST); mean of absolute value of distances from antero-posterior (mDISTap) and medio-lateral (mDISTml) axis of displacement series were computed. Frequency domain measures: Discrete Fourier Transform was computed for time domain measures. The whole periodogram was split in four spectral windows: $0-0.1 / 0.1-0.5 / 0.5-2 /$ 2-20 Hz for DIST, DISTap and DISTml. This choice made it possible to observe the subjects' strategies in AP and ML axes, in their long and short-loop reflex. Statistical analysis was conducted through ANOVA for repeated measures; the contrasts were performed comparing each time (from 1 to $24 \mathrm{~h}$ ) following with the values obtained in pre HIIT condition.

Results: HIIT test causes an initial increase in all the measured indices followed by a subsequent decrease. The trends of the indices measured in the time domain are not significant; this is mainly due to the great interpersonal variability and the high CV values. Instead in the frequency domain it is verified that the spectral windows related to the AP direction are significantly associated with the measurement time. Furthermore, even after $24 \mathrm{~h}$ some indices did not return to the pre-HIIT test values.

Conclusions: Fatigue affects the postural sway in a complex manner and complete recovery time varies between subjects.

Reference

Calavalle AR, Sisti D, Rocchi MBL, Panebianco R, Del Sal M, Stocchi V (2008) Postural trials: expertise in rhythmic gymnastics increases control in lateral directions. Eur $\mathrm{J}$ Appl Physiol 104:643-649 


\section{PO P}

\section{Effect of hyaluronic acid local injections on Achilles tendinitis: an observational study on tendon viscoelastic properties in recreational runners}

\author{
M. Gervasj, E. Barbieri, I. Capparucci, G. Annibalini, \\ S. Contarelli, D. Sisti, S. Amatori, V. Biancalana, \\ P. Sestili
}

Department of Biomolecular Sciences, University Urbino Carlo Bo, Italy

Purpose: Achilles tendinitis (AT) causes 5\% of professional athletes to end their careers; in Europe AT affects ca. 10 millions recreational runners. The aim of this study was to evaluate the clinical and functional efficacy of a three-local injections regimen of hyaluronic acid (HA) in ameliorating pain score and function in middle aged recreational runners with AT, whose functional and clinical parameters were recorded integrating multiple techniques.

Methods: 8 recreational runners previously diagnosed for monolateral AT were enrolled. AT was confirmed before the first local HA injection (T0) by clinical examination, MRI and thermography. At T0 patients were assessed for maximal voluntary isometric contraction (MVI) involving Achilles tendon (both injured and healthy), and pain level with a Likert scale; Achilles tendon viscoelastic state, i.e. tone and stiffness, were then measured at relaxed state and at $10 \%$ of MVI with MyotonPro (Myoton Ltd, UK). Finally patients received the first HA injection (RegenFlex T\&M, a blend of $2-1000 \mathrm{kDa} H A$, Regenyal, IT). All the measurements were repeated at T1 (15 days after the first injections and immediately prior the second), at T2 (15 days after the second injection and prior the third) and at T3 (15 days after the third injection), i.e. over a total of 45 days in which clinical visits were also performed. Furthermore, before each injection, injured tendon exudates were collected by needle aspiration.

Results: At T0, tone and stiffness values were significantly different between injured and contralateral tendons, especially when measured at the relaxed state. Pain score and MVI, coherently with clinical outcomes, were significantly higher and lower in injured tendons, respectively. Interestingly, the above differences gradually disappeared at T1, 2 and 3. In keeping with these results, tendon exudates volumes also decreased.

Discussion: A three-local RegenFlex T\&M injections treatment was effective in the management of AT, as determined with a multimethodological approach. In particular, this is the first study indicating that AT alters the viscoelastic features of the tendon. To this regard, the healing process promoted by RegenFlex T\&M is accompanied by recovery of tendon viscoelastic parameters.

Conclusions: RegenFlex T\&M promoted a progressive healing of AT, with recovery of clinical, functional and tendon's viscoelastic state.

\section{PO P}

How sports affect static baropodometry? An observational study carried out in women living in southern Italy

\author{
K. Feka ${ }^{1}$, G. Messina ${ }^{2}$, F. Pomara ${ }^{3}$, V. Giustino ${ }^{4}$, \\ A. Iovane ${ }^{4}$, M. Bellafiore ${ }^{4}$, G. Battaglia ${ }^{4}$, A. Palma ${ }^{4}$, \\ A. Bianco ${ }^{4}$
}

${ }^{1}$ Ph.D. Program in Health Promotion and Cognitive Sciences, University of Palermo, Palermo, Italy; ${ }^{2}$ POSTURALAB Italia, Palermo, Italy; ${ }^{3}$ MEDEOR Research Institute, Palermo, Italy; ${ }^{4}$ Sport and Exercise Sciences Research Unit, SPPF Department, University of Palermo, Italy

Purpose: The purpose of the current study was two-fold: (a) to investigate the relationship between plantar pressure and surface distribution between athletes and sedentary women; and (b) to see the differences, if any, in these parameters between two groups and also the differences between the sports within athletes' group.

Methods: One hundred and seventy-three (173) female participants were enrolled in this study, where ninety-eight (98) were sedentary (age: $24.23 \pm 6.11$ years; height $161.11 \pm 6.44 \mathrm{~cm}$; weight $56.70 \pm 8.19 \mathrm{~kg}$; BMI $21.81 \pm 2.52 \mathrm{~kg} / \mathrm{m}^{2}$; Body Surface Area (BSA) $1.59 \pm 0.13$; shoe nr. $37.83 \pm 1.53$ ) while seventy-five (75) were athletes (age $22.47 \pm 4.89$ years; height $159.98 \pm 5.95 \mathrm{~cm}$; weight $55.49 \pm 7.61 \mathrm{~kg}$; BMI $21.62 \pm 2.18 \mathrm{~kg} / \mathrm{m}^{2}$; BSA $1.57 \pm 0.12$; shoe nr38.05 \pm 1.55 ). For plantar support analysis, FreeMed posturography system was used, including the FreeMed baropodometric platform as well as FreeStep v.1.0.3 software. Orthostatic position was maintained on the baropodometric platform in order to detect differences

Results: No significant differences were found regarding anthropometric data (age, height, weight, BMI, BSA and shoe number). Moreover, no significant differences were detected in total surface, forefoot, rearfoot, total left and total right foot surface between sedentary and athletes, as well. In addition, significant differences between sedentary $(50.39 \pm 3.60 \% ; \quad 49.61 \pm 3.60 \%)$ and athletes $(52.36 \pm 3.76 \%$; $47.64 \pm 3.73 \%)$ were found in forefoot and rearfoot load $(P=0.0006$ and $P=0.0006$ ). Moreover, also the maximal peak pressure and the mean pressure resulted significantly different between groups (Sedentary vs Athletes). Of interest, there were also significant differences between sports (athlete group) in total surface, forefoot and rearfoot surface and total left and right surface and in some load pressure parameters.

Conclusions: We can conclude that women who practice sport differ from sedentary ones in forefoot-rearfoot ratio pressure. Moreover, we detected also plantar surface and forefoot-rearfoot ratio pressure differences within the athletes group.

Keywords: Sedentary, Athlete, Baropodometry, Plantar pressure distribution

References

1. Wong PL, Chamari K, Mao DW et al (2007) Higher plantar pressure on the medial side in four soccer-related movements. $\mathrm{Br}$ J Sports Med 41:93-100

2. Gravante G, Russo G, Pomara F et al (2002) Influence of physical activity on plantar pressures and on ground projection of the body barycentre. Med Sport 55:269-278

3. Russo G, Truglio G, Gravante G et al (1998) Analisi baropodometrica statica e dinamica in maratoneti e bodybuilders. Med Sport 51:299-305

4. de Paula Lima PO, Camelo PRP, Ferreira V et al (2017) Evaluation of the isokinetic muscle function, postural control and plantar pressure distribution in capoeira players: a cross-sectional study. Muscles Ligaments Tendons J 7:498-503 


\section{SOCIAL, ECONOMIC AND LEGAL AS- PECTS OF SPORT}

\author{
186 SEL $P$ \\ A new instrument in the fight against doping: \\ the Athlete Biological Passport ("ABP"). Light \\ and shade \\ P. Garraffa ${ }^{1}$ \\ ${ }^{1}$ Department of Juridical Sciences, Link Campus University, Catania, \\ Italy
}

Purpose: Introduced for the first time in Cycling in 2008-and subsequently adopted in other sports (such as athletics and football) the Athlete Biological Passport ("ABP") represents, today, the last 'frontier' in the fight against Doping in sports.

Passing through the failure of the WADA's 'testing' approach (made 'in' and 'out' of competitions) as 'direct' detection method of Antidoping Rules Violations (ADRV's), the ABP has been considered an 'indirect' method for ADRV's, although with a greater degree of credibility amongst the scientific community.

The aim of this work is to draw a first balance sheet of such an instrument, by highlighting its advantages and criticalities.

Methods: In an era of highly sophisticated scientific techniques, it is reasonable to ask how it could be possible that EPO's detection has been so problematic so far.

EPO (or "Erythropoietin", also known as "hematopoietin" or "hemopoietin") is a hormone naturally generated in adult kidneys, and is the body's principal regulator of the biological process responsible for the amount of circulatory red blood cells, being-as a matter of factone of the most diffused performance enhancing drugs used by the athletes for a long time.

In this work we will address the intrinsic difficulties arising from the "EPO-test" (a test designed for EPO's detection), the reasons why such a method has failed, and the ABP is considered, today, the more effective and trustworthy instrument for ADRV's detection.

The ABP consists in an individual, electronic record for each athlete, in which the results of all doping tests are collected in the framework of such a programme over a period of time.

It contains, in particular, an Hematological Profile (consisting of the combined results of hematological parameters, analysed in a series of blood samples), and a Steroid Profile (consisting of the combined results of steroid levels in a series of urine samples), and works through the identification of a longitudinal profile ("over the time") for professional athletes.

Results: Despite the undisputed improvements in the detection of cheating of doping in sports, such an instrument has triggered, nevertheless, a wide-ranging debate amongst the scientific community, also by going through the scrutiny of the highest-profile sports courts, including the CAS of Lausanne.

References

Garraffa P (2018) A new instrument in the fight against doping: the Athlete Biological Passport ("ABP"). Light and shade. Rivista Italiana di Medicina Legale, n. 1-2018, pp 71-91

Mason E (2013) The athlete biological passport: a 'magic bullet' for EPO detection? (http://www.lawinsport.com, 6 February 2013)

Moston S, Engelberg T (2017) Detecting doping in sport

\section{SEL $P$ \\ Sport as a mean for preventing violence and exclusion: the SAVE Project}

\author{
A. Gentile ${ }^{1}$, A. Bianco ${ }^{2}$, I. Valantine ${ }^{3}$, \\ D. Mujkic ${ }^{4}$, A. Palma ${ }^{2}$, S. Boca ${ }^{2}$
}

${ }^{1}$ Ph.D. Program in Health Promotion and Cognitive Sciences, University of Palermo, Palermo, Italy;

${ }^{2}$ Department of Psychological, Pedagogical and Educational

Sciences, University of Palermo, Palermo, Italy;

${ }^{3}$ Department of Sport Management, Economics and Sociology,

Lithuanian Sports University;

${ }^{4}$ Faculty of Sport ad Physical Education, University of Sarajevo, Sarajevo, Bosnia and Herzegovina

Purpose: The SAVE (Sport Against Violence and Exclusion) project is an Erasmus + Sport project co-founded by the European Union (Key Action: Sport590711-EPP-1-2017-1-LT-SPO-SCP), whose general objective is to prevent violent and socially exclusive behaviors in the sport field. Coaches from grass-root sport clubs, that train children from 6 years to 12 years old, will be involved in a pilot course for recognizing and managing violent behaviors and exclusion episodes (Gentile et al. 2018). According to the psychological literature, the sport environment is an eligible instrument able to foster relations among team members, which, in turn, should reduce the risk of deviant behaviors as violence (Hirschi 1998). Furthermore, children can learn while playing how to adapt their behavior to the social norms of a specific social setting.

Methods: The project structure is made up of nine tasks that have to be implemented at the same time in seven different European countries (Italy, Lithuania, Bosnia-Herzegovina, Serbia, Austria, Croatia, Spain). Through a process of collecting information from these countries (Desk Analysis and Needs Assessment-Task 1) about what is conceived "Violent" or "Socially Exclusive", together with scientific research (Field Research-Task 3), it will be possible to design a plan for developing Competence Card (Task 4) which is necessary to structure the Curriculum (Task 5) for the Piloting Phase (Task 6). In this phase, a group of coaches from the different consortium countries will be trained to acquire the competences needed to recognize violent behaviors or episodes of exclusion. Through the assessment of the Training Process (Task 7) and the Adaptation of the Training Material (Task 8), a Training KIT will be created (Task 9). The project will provide also a SAVE online Platform (Task 2) in which coaches can register their own sport club and find materials focused on the topic.

Results: The project should provide to the coaches a conflict resolution tool validated in several EU countries and should arise the sport clubs' interest in joining this initiative through their registration in SAVE online platform.

Conclusions: The SAVE project should fight against violence and exclusion by fostering children's moral conscience through the interactions with significant adults within the sport field (Gentile et al. 2018; Vygotskij 1934).

Keywords: Social inclusion, Aggressive behaviors, Conflict resolution tool, Cross-cultural research

\section{References}

Gentile A, Valantine I, Staskeviciute-Butiene I, Kreivyte R, Mujkic D, Ajdinovic A, Kezić A, Miletić Đ, Kovačević A, Madic D, Drid P, Bianco A (2018) Preventing violence and social exclusion through sport and physical activity: the SAVE Project. J Funct Morphol Kinesiol 3:25

Hirschi T (1998) Social bond theory. Criminological theory: past to present. Roxbury, Los Angeles

Vygotskij L (1934) Pensiero e linguaggio. Ricerche psicologiche 


\section{SEL $P$}

Analysis of sociodemographic and sports-related factors as correlates of potential doping behavior in high-level junior swimmers

D. Maric ${ }^{1,2}$, A. Bianco ${ }^{1}$, D. Sekulic ${ }^{2}$

${ }^{1}$ PhD Program in Health Promotion and Cognitive Sciences, Sport and Exercise Research Unit, SPPF Department, University of Palermo, Palermo 90144, Italy; dora.maric@unipa.it (D.M.); antonino.bianco@unipa.it (A.B.);

${ }^{2}$ Faculty of Kinesiology, University of Split, Split 21000, Croatia; dado@kifst.hr (D.S.)

Purpose: Doping behavior, including the misuse of performanceenhancing drugs, is currently a serious problem in sports, and the efficacy of preventive efforts directly depends on information regarding the associations among different precipitating factors (PF) and doping behavior. This study aimed to establish the PF of potential doping behavior (PDB) in high-level junior swimmers.

Methods: Study included 242 competitive swimmers from Slovenia both female and male, (131 females, $14.4 \pm 1.2$ years, 111 males, $15.3 \pm 1.1$ ), athletes were tested during 2017 National Championship and were under 18 years of age. Variables were collected by previously validated questionnaires, which included questions on sociodemographics, sports-related factors, and doping-related factors (Sekulic et al. 2016). The PDB (positive attitude, neutral attitude, or negative attitude toward doping) was observed as a criterion, while other variables were included as predictors in multinomial logistic regression analyses, which additionally controlled for gender as a confounder.

Results: The results have shown no significant difference between male and female susceptibility to doping. Competitive achievement in Olympic(neutral-attitude: 0.6 [0.43-0.83], positive-attitude: 0.61 [0.42-0.91]), and non-Olympic disciplines (positive-attitude: 0.56 [0.37-0.86]) was protective against PDB.

Conclusions: The results of the study provide more precise insight into the specific factors associated with PDB in swimming. The established precipitating factors should be incorporated into targeted anti-doping campaigns in this sport.

Reference

Sekulic D, Tahiraj E, Zvan M, Zenic N, Uljevic O, Lesnik B (2016) Doping attitudes and covariates of potential doping behaviour in highlevel team-sport athletes; gender specific analysis. J Sports Sci Med 15(4):606-615

\section{SUNDAY ORAL SESSION II}

\section{BIOMOLECULAR AND NUTRITIONAL ASPECTS OF EXERCISE AND SPORT 2}

189 BN2 K

Effects of the acute carnosine and ßalanine supplementation on the cardio-respiratory parameters during exhaustive cycle exercise

\author{
S. Shokohyar, E. Limonta, S. Rampicini, E. Ce, S. Longo, \\ G. Coratella, L. Galasso, F. Esposito
}

Department of Biomedical Sciences for Health, University of Milan, Milan, Italy

Purpose: Several studies investigated the ergogenic effects of $\beta$ alanine and carnosine supplementation on exercise performance after chronic supplementation. In contrast, very few studies investigated the acute effects of a single $\beta$-alanine and carnosine supplementation. Although a chronic intake is necessary to ensure a significant increase in muscular carnosine level, some evidence suggest that this dipeptide may already be available within muscle fibers few hours after ingestion. The purpose of this study was to verify the acute effect of carnosine and $\beta$-alanine supplementation on endurance exercise at low and high exercise intensities $(50 \%$ and $90 \%$ maximum oxygen uptake, $\mathrm{VO}_{2 \max }$ ).

Methods: Thirteen participants (age $22 \pm 1.8$ years, body mass $72.5 \pm 7.9 \mathrm{~kg}$, height $172 \pm 8 \mathrm{~cm}$, BMI $24.5 \pm 1.9 \mathrm{~kg} / \mathrm{m}^{2}$, fat mass $12.62 \pm 3.51 \%$ ) were recruited. Subjects performed 6 experimental sessions. During the first and second, a discontinuous incremental cycle ergometer tests (5 different workload each lasting 5 min with 5 min of rest in between), were performed under the two experimental conditions: preceded by placebo (Pla) or carnosine and $\beta$-alanine (Carn- $\beta$ a) supplementation. From this test, the load corresponding to $\mathrm{VO}_{2 \max }$ was obtained, from which $90 \%$ and $50 \%$ were calculated. In the other sessions, a constant-load exhaustive exercise at the two intensities were performed in both experimental conditions (Pla and Carn- $\beta \mathrm{a})$, in a random order. The supplementation provided $250 \mathrm{mg}$ of L-carnosine $+250 \mathrm{mg}$ of $\beta$-alanine administrated $4 \mathrm{~h}$ before tests. In Pla condition, analogues inert substances were taken. The main cardio-respiratory parameters $\left(\mathrm{VO}_{2} ;\right.$ carbon dioxide production, $\mathrm{VCO}_{2}$; respiration rate, $f_{\mathrm{R}}$; minute ventilation, $\mathrm{VE}$; tidal volume, VT; respiratory quotient, $\mathrm{R}$; heart rate, $f_{\mathrm{H}}$ ) were measured. Exercise efficiency (relationship between mechanical and metabolic work) was also calculated. The significance level was set at $\alpha \leq 0.05$.

Results: No significant differences were found in cardio-respiratory parameters between the two experimental conditions, both during the maximal square-wave tests and during the exhaustive constant load exercises at $50 \%$ and $90 \% \mathrm{VO}_{2 \max }$. Also exercise efficiency was not affected by acute Carn- $\beta$ a administration.

Conclusion: Overall, these findings suggest that a single supplementation of Carn- $\beta \mathrm{a}$, in the amount of $2.5 \mathrm{~g}$ of carnosine $+2.5 \mathrm{~g}$ of $\beta$-alanine, is not able to affect low and high levels of endurance cycle exercise performance. 


\section{$190 \mathrm{BN} 2 \mathrm{O}$}

Different amounts of protein intake influence body composition and performance in elite cyclists

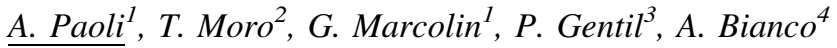 \\ ${ }^{1}$ Department of Biomedical Sciences, University of Padova, Padova; \\ ${ }^{2}$ Department of Nutrition and Metabolism, University of Texas \\ Medical Branch, Galveston, TX, USA; \\ ${ }^{3}$ College of Physical Education and Dance, Federal University \\ of Goias, Goiania, GO, Brazil; \\ ${ }^{4}$ Sport and Exercise Sciences Research Unit, University of Palermo, \\ Palermo, Italy
}

Purpose: The ideal amount of protein for endurance athletes has been poorly investigated.

The aim of our study was to evaluated the physiological impact of different dietary protein intakes on body composition and performance outcomes in a group of elite cyclists.

Methods: Thirty-four elite cyclists $(1600-1800 \mathrm{~km} / \mathrm{month})$ participated to the study. Subjects were divided in 4 groups with different levels of protein intake: normal (NP, $1.2 \mathrm{~g} / \mathrm{kg}$ ), moderate (MP, $1.6 \mathrm{~g} /$ $\mathrm{kg}$ ), high (HP, $2.0 \mathrm{~g} / \mathrm{kg}$ ) or very high (VHP $2.4 \mathrm{~g} / \mathrm{kg}$ )protein for 8 weeks. In the diets fats were maintained constant whilst energy from carbohydrate and protein was modified to maintain an isocaloric diet. Body composition was assessed via Dual X Ray Absorptiometry (DXA) and via ultrasound to calculate cross sectional area (CSA) of the anterior thigh. $\mathrm{VO}_{2} \max$, peak power output and $1 \mathrm{RM}$ half squat test were also performed. Moreover we executed routinely blood exams.

Results: After 2 months both HP and VHP showed a significant improvement of $1 \mathrm{RM}$ (HP pre $133 \pm 14 \mathrm{~kg}$ vs. post $141 \pm 12 \mathrm{~kg}$, $\mathrm{p}<0.001$; VHP pre $137 \pm 12 \mathrm{~kg}$ vs. post $144 \pm 11 \mathrm{~kg}, \mathrm{p}<0.001)$, PPO (HP pre $505 \pm 78 \mathrm{~W}$ vs. post $534 \pm 67 \mathrm{~W}, \mathrm{p}<0.001$; VHP pre $512 \pm 55 \mathrm{~W}$ vs. post $541 \pm 76 \mathrm{~W}, \mathrm{p}<0.001$ ), and VO2max (HP pre $62.1 \pm 5.8 \mathrm{mlO}_{2} / \mathrm{kg}$ vs. post $64.5 \pm 5.9 \mathrm{mlO}_{2} / \mathrm{kg}, \mathrm{p}<0.001 ; \mathrm{VHP}$ pre $61.2 \pm 5.5 \mathrm{mlO}_{2} / \mathrm{kg}$ vs. post $\left.64.1 \pm 7.6 \mathrm{mlO}_{2} / \mathrm{kg}, \mathrm{p}<0.001\right)$, without differences between groups. There were no significant changes of $1 \mathrm{RM}$ and VO2max for both NP and MP whilst NP showed a slight but significant decrease of PPO. Both HP and VHP showed a significant increase of lean body mass (LBM) (HP pre $64.72 \pm 1.9 \mathrm{~kg}$ vs. post $65.99 \pm 2.2 \mathrm{~kg}, \mathrm{p}<0.001$; VHP pre $65.52 \pm 2.0 \mathrm{~kg}$ vs. post $67.61 \pm 1.7 \mathrm{~kg}$ ) whilst both NP and MP showed a significant decrease (NP pre $63.31 \pm 2.1 \mathrm{~kg}$ vs. post $62.4 \pm 2.3, \mathrm{p}<0.05$; MP pre $66.88 \pm 1.8 \mathrm{~kg}$ vs. post $65.80 \pm 2.9)$. HP and VHP showed a significant increase of anterior thigh CSA (HP pre $50.5 \pm 7.8 \mathrm{~cm}^{2}$ vs. post $53.4 \pm 6.7 \mathrm{~cm}^{2}, \mathrm{p}<0.001$; VHP pre $51.2 \pm 5.5 \mathrm{~cm}^{2}$ vs. post $54.1 \pm 7.6 \mathrm{~cm}^{2}$ ). No changes of blood values were detected.

Conclusions: Taken together our data suggest that an higher protein intake ( 2.0 and $2.4 \mathrm{~g} / \mathrm{kg}$ ) may help elite cyclists to improve performance and to increase muscle mass without differences between the two levels of protein intake. Instead 1.2 and $1.6 \mathrm{~g} / \mathrm{kg}$ of protein seemed to be not sufficient and could impair performance and muscle mass. Future studies directed at mechanisms by which level of protein intake influences performance outcomes are needed.

\section{BN2 O \\ Water Polo players's era induce AMPK $\alpha / A C C$ activation status on hepatoma cells line}

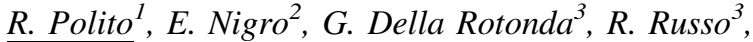 \\ A. Daniele $e^{1}$
}

${ }^{I}$ Dipartimento di Scienze e Tecnologie Ambientali, Biologiche, Farmaceutiche, Università della Campania "Luigi Vanvitelli", Caserta, Italy;

${ }^{2}$ Dipartimento di Scienze Cardio-Toraciche e Respiratorie, Università degli Studi della Campania, "Luigi Vanvitelli", Via Leonardo Bianchi clo Ospedale Mondaldi, 80131 Naples, Italy; ${ }^{3}$ Ospedale Pineta Grande, 81030 Pineta Grande, Caserta, Italy

Purpose: Physical activity has an important role in the prevention of many chronic diseases such as metabolic syndrome, type 2 diabetes mellitus and liver diseases. Regular physical activity indeed increases energy expenditure, reduces fat depots, promotes beta-oxidation and modulates cytokines production. On the other hand, obesity and insulin resistance are very often associated with the development of liver diseases. The aim of this study is to investigate the effects of elite athletes' sera (water polo), basket players and obese sera before and after weight loss on a hepatoma cell line, Hep G2 cells.

Methods: We treated Hep G2 cells with $10 \%$ sera and analyzed: cell viability through MTT assay, activation status of AMPK $\alpha$ and ACC through western blotting; in addition, serum cytokines levels in sera from WP players were analyzed through ELISA assay.

Results: We found that cell viability is not affected by the treatment with sera with any differences among the various treatments. In cells treated with WP players' sera, the phosphorylation of AMPK $\alpha$ statistically increases compared to cells treated with control subject's sera. Regarding the phosphorylation status of ACC, WP players' sera induce an increase in the phosphorylation of ACC compared to cells treated with control subject's, obese before and after weight loss and basket players sera even if it is statistically significant. Furthermore, we analyzed serum levels of the several cytokines in sera from WP players and compared it to controls. We found that IL-2, IL-4, IL-6 and IL-8 levels are increased in athlete's sera than in control subjects. Similarly, IL-10 serum levels are significantly higher in WP Players compared to controls. TNF- $\alpha$ and INF- $\gamma$ are not differentially expressed between athletes and controls.

Conclusions: We demonstrated that an intense physical activity induces activation of AMPK $\alpha$ and ACC suggesting that physical activity could be considered as a powerful physiological activator of AMPK $\alpha$. These findings reinforce the idea that the activation of these mediators are useful for the management of metabolic hepatic disorders. 


\section{BN2 O}

Effect of a carbohydrate/branched chain aminoacids/ alanine sport nutritional Supplement (friliver ${ }^{\circledR}$ performance) on perceived exertion in a 9-weeks high intensity interval training sessions

M. Gervasi ${ }^{1}{ }^{1}$, D. Sisti ${ }^{1}$, S. Amatori ${ }^{1}$, A.R. Calavalle ${ }^{1}$,

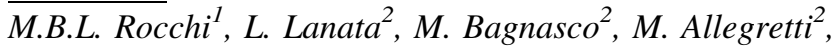
P. Sestili ${ }^{1}$

${ }^{1}$ Department of Biomolecular Sciences, University of Urbino, Italy; ${ }^{2}$ Dompé Farmaceutici Spa, Milano, Italy

Purpose: Branched chain aminoacids (BCAA) have been reported to increase tissue proteins' synthesis during and after exercise and to speed up the metabolism. Recent studies demonstrate that BCAA administration before endurance exercise improve psychological aspects connected to fatigue. Here we sought to determine, in a randomized double-blind placebo-controlled study, whether the intake of a carbohydrate/BCAA/alanine supplement, affects the rating of perceived exertion (RPE) during high intensity interval training (HIIT) sessions.

Methods: 32 healthy subjects, performed a 9-week indoor cycling training consisting in 36 sessions. One $\mathrm{h}$ before the tests of each training session, the subjects of the verum group (TR) ingested a single dose of Friliver ${ }^{\circledR}$ Performance in accordance with manufacturer's recommendations, while those of the placebo group (PL) ingested a noncaloric powder identical in taste and appearance. Before (PRE) and after (POST) training, participants underwent a HIIT cycling test consisting of ten $90 \mathrm{~s}$ sprints (SPR) at $90 \%$ of individual maximal power (Pmax) separated by $180 \mathrm{~s}$ recovery intervals (REC) at 55\% Pmax. RPE (CR-10) was assessed during the HIIT test, $10 \mathrm{~s}$ before the end of each step. Percent variations of RPE between TR and PL were then analyzed during pretraining SPR and REC steps and post-training SPR and REC steps.

Results: In the pre-training test, the Delta Mean RPE \% (TR vs. PL) was $1 \%$ during SPR HIIT and 9\% during REC HIIT, with a Delta Max RPE of 5\% and 15\%, respectively. In the post-training test, after the 9-week physical training, the Delta Mean RPE \% (TR vs PL) was $13 \%$ during SPR HIIT and $21 \%$ during REC HIIT, with the Delta Max RPE of $16 \%$ and $26 \%$, respectively. Both single and long-term administration of Friliver ${ }^{\circledR}$ Performance significantly reduced RPE compared to placebo.

Conclusion: Treatment with Friliver ${ }^{\circledR}$ Performance over a 9-week physical training significantly reduces mean perceived exertion during SPR and REC steps. The results of our study suggest that a 9-week training program with Friliver ${ }^{\circledR}$ Performance intake may help athletes to sustain training and optimize their performance.

\section{BN2 O}

12 weeks effects of an Essential Amino Acids (EAA)based multi-ingredients supplement with vitamin $D$, creatine, antioxidants and magnesium on aging frailty markers. A randomized controlled double-blind study

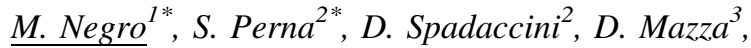
L. Calanni $^{1}$, M. Rondanelli ${ }^{2}$, G. D'Antona ${ }^{1}$

${ }^{1}$ CRIAMS-Sport Medicine Centre, University of Pavia, Voghera, Italy;

${ }^{2}$ Department of Public Health, Experimental and Forensic Medicine, School of Medicine, Endocrinology and Nutrition Unit, University of Pavia, Azienda di Servizi alla Persona di Pavia, Pavia, Italy; ${ }^{3}$ S.I.I.T. S.r.l. Pharmaceutical \& Health Food Supplements Contract Manufacturing, Trezzano S/N, Italy

$\dagger^{* *}$ These authors contributed equally to this work.

Purpose: The aim of the present study was to evaluate the efficacy of twice daily consumption of a essential amino acids (EAA)-based multi-ingredient nutritional supplement (SUPP), compared to placebo (PLA), on body composition, body hydration, muscle strength, muscle power and 25-hydroxyvitamin D blood level in healthy elderly subjects.

Methods: 38 subjects ( $69 \pm 5$ years; 8 men and 30 women) were randomized. Dual Energy X-Ray Absorptiometry (DXA) was used for quantify Free Fat Mass (FFM), Appendicular Lean Mass (ALM), Appendicular Lean Mass index $\left(\mathrm{ALM} / \mathrm{H}^{2}\right)$, Fat Mass (FM), Visceral Adipose Tissue (VAT). Body Hydration (Total Body Water, TBW; Intra-Cellular Water, ICW; Extra-Cellular Water, ECW) was determined by Bioelectrical Impedance Analysis (BIA). Biceps brachii Muscle Pick Power (MPP) and Muscle Voluntary Contraction (MVC) were measured by a force-velocity device analysis and isometricergometer, respectively. Blood measurement of 25-hydroxyvitamin D was assessed by fasting venous samples.

Results: 31 subjects completed the study and 7 dropped out $(n=2$ in SUPP and $n=5$ in PLA group, respectively). After 12 weeks of intervention, significant increases in SUPP group were found and summarized as follow: (1) vitamin D (+ 8.73; p < 0.001); (2) ALM: $+0.34 \mathrm{~kg}$ and $\left.\mathrm{ALM} / \mathrm{H}^{2}:+0.12 \mathrm{~kg} / \mathrm{m}^{2} ; \mathrm{p}<0.001\right)$; (3) MPP $(+4.82 \mathrm{~W} ; \mathrm{p}<0.001)$ and MVC $(+0.52 \mathrm{~kg} ; \mathrm{p}<0.001)$. Furthermore, a significant decrease of VAT $(-76.25 \mathrm{~g} ; \mathrm{p}<0.001)$ was detected in SUPP. The results of intergroup analysis (SUPP vs. PLA) showed improvements: (1) of vitamin D level $(+11.72 \mathrm{ng} / \mathrm{ml}$; $\mathrm{p}<0.001)$; (2) of leg FFM response $(+0.5 \mathrm{~kg} ; \mathrm{p}<0.05)$; (3) of $\operatorname{ALM}(+0.53 ; \mathrm{p}<0.05)$ and $\mathrm{ALM} / \mathrm{H}^{2}\left(+0.19 \mathrm{~kg} / \mathrm{m}^{2} ; \mathrm{p}<0.05\right) ;(4)$ of biceps brachii MPP $(+9.87 \mathrm{~W} ; \mathrm{p}<0.05)$ and MVC $(+1.38 \mathrm{~kg}$; $\mathrm{p}<0.05$ ). In treatment group a significant correlation was found between: vitamin $\mathrm{D}$ and $\mathrm{ALM} / \mathrm{H}^{2}(\mathrm{r}=0.706 ; \mathrm{p}<0.001)$, VAT/FM and MVC ( $\mathrm{r}=-0.572 ; \mathrm{p}<0.001)$, ICW and MVC $(\mathrm{r}=0.533$; $\mathrm{p}<0.05)$, leg FFM and $\mathrm{ALM} / \mathrm{H}^{2}(\mathrm{r}=0.857 ; \mathrm{p}<0.001)$.

Conclusions: The study demonstrate that an EAA-based multi-ingredient nutritional supplement can affect muscle mass, muscle function and metabolic conditions related to muscle health in older adults. Furthermore, these findings are very promising because of they were assessed in medium-short period of time and in a setting of nutritionally controlled healthy elderly. 


\section{SPORT BIOMECHANICS AND TECHNOL- OGY 3}

\section{SB3 K \\ Anthropometric characteristics for identifying personalized anterior-posterior block distances in sprint start}

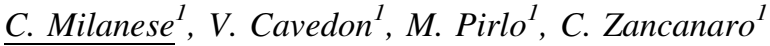 \\ ${ }^{1}$ Department of Neurosciences, Biomedicine and Movement Sciences, University of Verona, Italy}

Purpose: The technique used at the starting block is greatly influenced by the setting of the block positions [1]. This study had a twofold purpose, firstly to test the effect of two block setting conditions on the kinematic and kinetic parameters of sprinters; secondly, by dividing the sprinters according to their Cormic Index, we investigated whether performance outcomes in the two block setting conditions were affected by the sprinter's physical shape.

Methods: Forty-two well-trained sprinters performed a total 6 of maximal-effort $10 \mathrm{~m}$ sprints using two starting conditions namely, the sprinter's usual personal block setting (UC) and a test condition (TC) where the blocks position was set according to a proportion of the sprinter's leg length. Dynamometric sprint blocks were used to measure the magnitude of forces generated by sprinters. The participants' Cormic Index ([sitting height/standing height] $\times 100)$ was calculated

Results: The anterior-posterior block distances were significantly different between UC and TC $(\mathrm{P}<0.001)$. TC induced a significant improvement in some kinematic and kinetic parameters e.g., the horizontal block velocity, the rear peak force, the rear force impulse, the total force impulse, the first and second step length and the time at $5 \mathrm{~m}$ and $10 \mathrm{~m}(\mathrm{P}<0.05 \div<0.001)$. When results were analyzed according to Cormic Index, TC induced a significant increase in the rear peak force and the rear force impulse $(\mathrm{P}<0.001)$ vs. UC for the metricormic group as well as an increase in the rear peak force in the brachycormic group $(\mathrm{P}=0.001)$ with no difference in the two conditions for the macrocormic group. Using anthropometry to set the starting blocks showed an effect in improving block starting performance in sprinters, in particular for the rear leg. Such an effect was most expressed in metricormic sprinters.

Reference

1. Slawinski J, Dumas R, Cheze L, Ontanon G, Miller C, MazureBnnefoy A (2012) 3D kinematic of bunched, medium and elongated sprint start. Int J Sports Med 33:555-560

Keywords: Sprint running, Set position, Biomechanics, Well-trained athletes, Cormic Index

\section{SB3 O \\ Infrared thermography evaluation of exercise in weightlifters: a preliminary study}

\author{
G. Epasto $^{1}$, E. Guglielmino ${ }^{1}$, L. Magaudda ${ }^{2,3}$, M. Righi $^{2}$, \\ R. Sorrentino ${ }^{3}$ \\ ${ }^{1}$ Department of Engineering, University of Messina; \\ ${ }^{2}$ Department of Biomedical and Dental Sciences \\ and Morphofunctional Imaging, University of Messina; \\ ${ }^{3}$ MS in Theory and Methods of Preventive and Adapted Physical \\ Activities, University of Messina
}

Purpose: The application of Infrared Thermography (IRT) in sport science is growing more and more, especially thanks to the technological evolution of the IR cameras and data processing software. In sport science, IRT is mainly applied to detect thermoregulation processes $[1,2]$, thermal asymmetries in athletes due to their individual characteristics [3], thermal modifications due to pain [4] or to endurance exercises [5].

This research aimed to measure athlete skin temperature during the phases of exercise in weightlifting. More in detail, thermograms were acquired during pull and drop phases in order to evaluate muscular response and possible technical inaccuracies.

Methods: The training of a volunteer male weightlifter (age 21 years, height $1.80 \mathrm{~m}$, body mass $80 \mathrm{~kg}$ and fat mass $11 \%$ ) was recorded by means of an IR camera (Flir Systems SC640). The camera-athlete distance was changed during the study to have different fields of view. Results: By analyzing IR videos of the pull, a temperature rise in the left biceps femoris compared to the right one was detected, maybe due to a small injury in the right knee. In the IR videos of the drop phase, a slight temperature difference was observed between pectoralis major muscles, without technical inaccuracy due to asymmetries.

Conclusions: It can be concluded that IRT is an effective tool in sport science and together with other diagnostic tools, can allow the evaluation of post-injury recovery of athletes.

Acknowledgments: The research reported in this paper was conducted with the facilities of the Research Project PONa3_00422 "CERISI" (Research and Innovation Centre of Excellence for Structure and Infrastructure of Large Dimensions).

\section{References}

1. Merla A, Mattei PA, Di Donato L, Romani GL (2010) Thermal imaging of cutaneous temperature modifications in runners during graded exercise. Ann Biomed Eng 38:158-163

2. Formenti D, Ludwig N, Gargano M, Gondola M, Dellerma N, Caumo A, Alberti G (2013) Thermal imaging of exerciseassociated skin temperature changes in trained and untrained female subjects. Ann Biomed Eng 41:863-871

3. del Estal A, Brito CJ, Galindo VE, de Durana ALD, Franchini E, Sillero-Quintana M (2017) Thermal asymmetries in striking combat sports athletes measured by infrared thermography. Sci Sports 32:e61-e67

4. Rossignoli I, Fernández-Cuevas I, Benito PJ, Herrero AJ (2016). Relationship between shoulder pain and skin temperature measured by infrared thermography in a wheelchair propulsion test. Infrared Phys Technol 76:251-258

5. Drzazga Z, Binek M, Pokora I, Sadowska-Krępa E (2018) A preliminary study on infrared thermal imaging of cross-country skiers and swimmers subjected to endurance exercise. J Therm Anal Calorim. https://doi.org/10.1007/s10973-018-7311-y 


\section{SB3 O}

\section{Performance evaluation in Compak Sporting} competition

\author{
R. Monzoni ${ }^{1}$, B. Lonzi ${ }^{2}$, F. Mari ${ }^{2}$, L. Banci ${ }^{2}$, \\ M. Vignaroli ${ }^{2}$, A. Federici ${ }^{1}$
}

${ }^{1}$ Department of Biomolecular Sciences, Division of Exercise and Health Sciences, University of Urbino Carlo Bo, Urbino, Italy; ${ }^{2}$ Department of Testing and Prototyping, Benelli Armi S.p.a., Urbino, Italy

Purpose: Clay pigeon shooting is a sport that requires dexterity and medium intensity muscular effort (Dal Monte 1983) where the shooters, using sportive shotguns, attempt to break clay targets mechanically flung into the air.

The aims of this study were to investigate the performance in Compak Sporting (FITASC 2016) competition and to evaluate pain and effort perceived by the shooters due to the recoil, using two sportive shotguns (shotgun_A, shotgun_B) with similar configurations but different technical characteristics (balance and ergonomics).

Methods: 8 shooters (age $26.8 \pm 4.6$ years; BMI $22.9 \pm 1.5 \mathrm{~kg}$ / $\mathrm{m}^{2}$ ) with at least 4 years of shooting experience were recruited. Each shooter have shot with both sportive shotguns.

Qualitative (Visual Analog Scale, VAS, and Rate of Perceived Exertion scale, RPE) and quantitative (Hand Grip Test dominant, HGTdh, and non-dominant hand, HGTndh) tests are proposed before (t0) and after competition (tf). VAS and RPE were used to evaluate pain and effort perceived after competition (tf), HGT of dominant and non-dominant hand were used to evaluate the muscular force variation between before (t0) and after competition (tf).

IntraClass Correlation (ICC) was used to exclude possible variations of shooters in rest condition ( $\mathrm{t} 0$ ) during the two competition days.

In order to assess possible differences between shotgun_A(sA) and shotgun_B(sB), the total scores of Compak Sporting competition and the results of the evaluative tests after competition (tf) were compared using a 2-tailed paired sample $t$ test. Significance was set a priori at $\mathrm{p}<.05$ for all tests.

Results: Based on the proposed protocol, the following results were obtained for the parameters of interest: score (sA $51.63 \pm 4.81, \mathrm{sB}$ $47.75 \pm 4.50$ ); VAS (sA $4.47 \pm 3.68, \mathrm{sB} 6.37 \pm 4.89$ ); RPE (sA $1.94 \pm 1.37, \mathrm{sB} 2.50 \pm 2.28) ;$ HGTdh $(\mathrm{sA}-0.73 \pm 0.18, \mathrm{sB}$ $-1.03 \pm 0.27)$ and HGTndh (sA $-3.16 \pm 0.74)$, sB $-5.16 \pm 0.79)$

The use of two different shotguns resulted in significant variations in handgrip strength $(\mathrm{p}=0.0001)$ : the grip of non-dominant hand of the shooters after firing with $\mathrm{sB}$ is weaker than when using sA.

Conclusion: This protocol has allowed to realize a method of performance evaluation through the analysis of the score, the reduction of the district muscular strength (upper arms) and effort and pain perceived by the shooters after competition.

References

Dal Monte A (1983) La valutazione funzionale dell'atleta. Sansoni Editore, Firenze

FITASC (2016) Regolamento internazionale Compak Sporting

Keywords: Score evaluation, Sports application, Compak sporting, Clay shooting

\section{SB3 O} Acute effects of non-customized, boil-and-bite oral
splint on muscle flexibility and dynamic balance

\author{
S. Rampichini, E. Limonta, M. Borrelli, E. Cè, S. Longo, \\ C. Doria, S. Shokohyar, G. Coratella, E. Roveda, \\ A. Montaruli, F. Esposito
}

Department of Biomedical Sciences for Health, Università degli Studi di Milano, Italia

Purpose: Recent studies concerning the effects of non-customized oral splints (boil-and-bite) on physical performance, indicate that these devices could improve muscle strength capacity. In particular, an increase in maximum force production, endurance time and force stability in the upper limb muscles was reported. While force capacity has been already investigated, the effects of the use of oral splints on muscle flexibility and coordinative aspects have not yet been elucidated. Therefore, this study aimed at analyzing the influence of oral splints on muscle flexibility and on dynamic balance.

Methods: Twelve amateur sports participants (age $37 \pm 4$ years; body mass $54 \pm 5 \mathrm{~kg}$; stature $1.66 \pm 0.06 \mathrm{~m}$; mean $\pm \mathrm{SD}$ ) were recruited. Flexibility tests included the sit-and-reach test, the upper limbs circling and the lower limbs abduction. Joint range of motion was also evaluated. The time to exhaustion in maintaining the forearm plank position was measured for evaluating strength capacity. Lastly, participants' balance was determined by measuring the displacement (frontal shift and spin degrees) after a Fukuda step test. Each test was performed with $(\mathrm{OS})$ and without $(\mathrm{CON})$ boil-and-bite oral splints (BRUX Power), in a random order.

Results: Upper limbs circling (107 $\pm 4 \mathrm{~cm}$ and $104 \pm 4 \mathrm{~cm}$ in CON and OS, respectively) and lower limbs abduction tests $\left(78 \pm 2^{\circ}\right.$ and $80 \pm 2^{\circ}$ in CON and OS, respectively) improved significantly when performed with OS. Equally, the time to exhaustion in the plank position ( $100 \pm 18 \mathrm{~s}$ and $114 \pm 16 \mathrm{~s}$ in CON and OS, respectively) increased significantly with the splint, and the spin degree of Fukuda test decreased $\left(81 \pm 36^{\circ}\right.$ and $54 \pm 30^{\circ}$ in CON and OS, respectively), although not significantly.

Conclusions: These results suggest that, even in acute condition, OS usage seems to influence positively muscle flexibility, isometric strength and dynamic balance. Similarly to what has been done in this study, future works should investigate the effect on dynamic balance and on flexibility after chronic use of non-customized oral splints.

\section{Reference}

Limonta E, Arienti C, Rampichini S, Venturelli M, Cè E, Veicsteinas A, Esposito F (2018) Effects of two different self-adapted occlusal splints on electromyographic and force parameters during elbow flexors isometric contraction. J Strength Cond Res 32(1):230-236 


\section{SB3 O}

Sex differences in upper and lower body muscular engagement during cross-country skiing

\section{Zoppirolli, B. Pellegrini R. Modena, A. Fornasiero, A, Savoldelli, F. Stella, L. Bortolan}

CeRiSM (Research Centre of Mountain Sport and Health), University of Verona, Rovereto, Italy; Department of Neuroscience, Biomedicine and Movement, University of Verona, Verona, Italy

Purpose: In cross-country skiing propulsive actions are exerted through skies and poles, thus involving upper body to an extent that depends on the specific technique performed. Since sex differences in endurance performance are greater when upper-body involvement is high, our aim was to verify if muscular engagement is sex dependent, when skiing at similar metabolic intensities using techniques requiring whole body involvement.

Methods: 12 male (M) and 8 female (F) high-level skiers performed a roller-skiing treadmill test to exhaustion by using the diagonal stride technique (@10 and $9 \mathrm{~km} \mathrm{~h}^{-1}$ for $\mathrm{M}$ and F, respectively; $1^{\circ}$-increments in slope every $3 \mathrm{~min}$ starting from $2^{\circ}$ ). The metabolic commitment for each step was estimated by oxygen consumption during the final 30-s of each step and cumulated blood lactate concentration. Correspondingly, the EMG activity of highly involved muscles belonging to different body compartments was measured to obtain the mean activation of each muscle, expressed as a percentage of MVC and to estimate cycle frequency.

Results: Test time and $\mathrm{V}^{\prime} \mathrm{O}_{2}$ max $\mathrm{kg}^{-1}$ were significantly higher in $\mathrm{M}$, but no differences were found in total metabolic work of sub-maximal steps $\left(2^{\circ}-9^{\circ}\right)$. EMG data revealed no sex differences for cycle frequency, trunk muscles and thigh extensors involvement (all $\mathrm{P}>$.050). F showed lower activations for triceps brachii $(-48 \%$, $\mathrm{P}=.024)$ and higher for rectus femoris muscle $(+35 \%, \mathrm{P}=.044)$ than $\mathrm{M}$.

Conclusions: When skiing at similar metabolic efforts and movement frequency, female skiers compensate the lower contribution of upperlimbs through an higher lower-limb involvement. This findings suggest that a reduced fractional utilization of upper-limb muscles strength during sport-specific contractions is another factor limiting performance in female skiers, together with the already known lower upper-body muscle mass and maximal strength capacity, respect to men. Additional strength-specific-dynamic training and technical improvements should be consider in high-level female skiers, to properly engage upper-limb muscles in propulsion while skiing.

Reference

Sandbakk O, Ettema G, Holmberg HC (2014) Gender differences in endurance performance by elite cross-country skiers are influenced by the contribution from poling. Scand J Med Sci Sports 24(1):28-33
POSTURAL APPROACH TO SPORT AND EXERCISE

199 PO K

Case report: evaluation of proprioceptive program in a patient with cerebellar ataxia after traumatic brain injury

G. Barone ${ }^{1}$, L. Zambito Marsala ${ }^{2}$, M. G. Benedetti ${ }^{3}$, L. Bragonzoni ${ }^{1,2}$

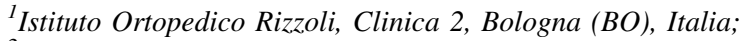

${ }^{2}$ Università di Bologna, Bologna (BO), Italia;

${ }^{3}$ Istituto Ortopedico Rizzoli, Medicina fisica e riabilitativa, Bologna (BO), Italia

Purpose: Traumatic brain injury (TBI) is one of the main causes of morbidity and mortality in the world [1]. Proprioception has a significant role in rehabilitation to promote and improve a correct postural control. The aim of this study is to evaluate the effect of proprioceptive training in a patient with cerebellar ataxia after traumatic brain injury using Delos Postural Proprioceptive System (Delos).

Methods: The subject is a male, 20 years old with a TBI, brought about severe cerebellar ataxia, weighty gait and postural control disorder. Patient walks with the aid of a stick and shows uncoordinated gait. The patient leads an active lifestyle: water and postural rehabilitation, competitive climbing and amateur thai boxe. The proprioception has been evaluated using static Riva test (single stance) and stabilometric test (double stance) by Delos System (Delos S.r.l-CTorino). The Delos System allows measuring quantitatively the proprioceptive control with visual feedback. The training consisted of different protocols, performed 2 days a week $\left(45^{\prime}-60^{\prime}\right)$ for 7 weeks. We compared the proprioceptive control before and after the protocol training. To measure the level of proprioceptive control in single leg stance exercises, we used stability index (SI) (percentage score, $100 \%$ is the theory test performed with maximal stability).

Result: In open eyes tests, SI improve of $7.1 \%$ in the left limb and $27.3 \%$ in the right one. In close eyes tests, SI improved in left and right limbs respectively of $51.6 \%$ and $28.8 \%$. Hands support decrease of $70 \mathrm{pp}$ in the left limb and $46 \mathrm{pp}$ in right limb. Visual dependence decrease of $17.4 \%$. After training, the variations of the centre of mass were smaller than pre-training once.

The final tests show a higher stability index. The patient reduced his support base and was able to perform final tests with heels together. During the first week of training, the patient needed double hands support in single stance training, instead, in the last session, he 
performed the tests with single hand support. The proprioceptive protocol will be revise according to the patient's improvements.

\section{Reference}

1. Hyder AA, Wunderlich CA, Puvanachandra P, Gururaj G, Kobusingye OC (2007) The impact of traumatic brain injuries: a global perspective. NeuroRehabilitation 22(5):341-353

Keywords: Proprioception, Ataxia, Traumatic brain injury

\section{PO O}

\section{Neural mobilization exercise for improve quality of life in patients with cervico-brachial pain.}

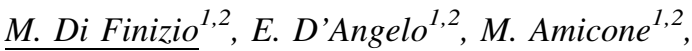 \\ M.G. Vinciguerra ${ }^{2}$ \\ ${ }^{1}$ Training Lab Italia, Independent Research Laboratory, Pescara, \\ Italy; \\ ${ }^{2}$ Scienze Motorie DISCAB, Università degli Studi L'Aquila, Coppito, \\ Italy
}

Purpose: Aim of the study was to investigate the efficacy of neural glides exercises on the symptoms of the cervico-brachial pain. Neural mobilization is called to affect the axoplasmic flow, movement of the nerve and its connective tissue. Furthermore the circulation of the nerve and dispersion of intraneural edema is affected by this principle. Neural mobilization decreases the excitability of dorsal horn cells and seems to be helpful to the mechanical and neurophysiological nerve function.

Methods: A 47-year-old man with a body mass index of $28 \mathrm{~kg} / \mathrm{m}^{2}$ has been submitted to neural mobilization training plan (NEURAL GLIDES) at Training Lab Italia, Fitness Centre and Independent Laboratory Research specialized on treatment of chronic diseases, consisted of 5 sets 12 reps with $90 \mathrm{~s}$ of rest for 8 weeks of sliders technique. Pain was quantified by the Numeric Rating Scale (NRS) ranging from 0 (no pain) to 10 (worst imaginable pain) and the short Form health Survey (SF-36).

Results: There was a significantly greater reduction in pain (NRS) after 8 weeks of treatment $(p<0.05)$, and the quality of life has improved.

Conclusions: Our study suggests that the neural mobilization exercise techniques could reduce cervico-brachial pain and also improve quality life. To reinforce our results we should expand the sample and compare our findings.

\section{Reference}

Basson et al (2015) The effectiveness of neural mobilizations in the treatment of musculoskeletal conditions: a systematic review protocol. JBI Database Syst Rev Implement Rep 13(1):65-75

\section{PO O \\ Modifications of balance and posture in people with facial piercings}

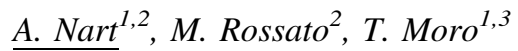

${ }^{I}$ Department of Biomedical Sciences, University of Padova, Padova Italy;

${ }^{2}$ Department of Biomolecular Sciences, University of Urbino, Urbino Italy;

${ }^{3}$ Department of Nutrition and Metabolism, UTMB, Galveston Texas

Purpose: Body art is a very interesting topic but its effects on posture has not been well establish yet. The aim of this study was to investigate the effects of piercing usage on balance and performance in young adults.

Methods: The study included 11 participants (SG) (8 females; 3 males), between the ages of 20 and 37 (25.04 \pm 4.71 years). Subjects were studied via electronic stabilometry in static mode, open and closed eyes, in the modalities piercing, without piercing and with plastic piercing. Results were then compared to the published guidelines (CG) (Rossato et al. 2013). Parameters of galvanic current recorded of any metal, were included in the analysis. At the same way a test to measure the range of the head rotation (subjective and objective evaluation) with and without metals was performed.

Results: The $t$ Student test showed that the Surface parameter, open and closed eyes, was statistically different from the CG $(\mathrm{p}<0.05)$, without gender and age distinction. Subsequently only a female group (FSG) (age between 20 and 29 years) was evaluated; the t-Student test has underlined a statistical difference for the parameters Ymoyen and AVD, open and closed eyes $(p<0.05)$ and for the parameters AVG and TalD, open eyes $(\mathrm{p}<0.05)$.

Conclusions: Some particular variables are not often considered in the imbalance of the postural adaption. The presence of piercings is a variable that could influence the proprioceptive training for the wellbeing improvement of the person.

\section{References}

Baranik M, Ciszewky A, Urbanek BM (2007) Corrosion by galvanic coupling between amalgam and different chromium-based alloy. Dental Mater 23:1256-1261

Fransson PA, Johansson R, Magnusson M (1995) Galvanic vestibular stimulation for analysis of postural adaptation and stability. IEEE Trans Biomed Eng 42:282-292

Rossato M, Bourgeois P, Ouaknine M (2013) Stabilometry standard guidelines 2011-2013 during clinical practice. Marrapese editore, Roma

Thyrion C, Roll JP (2010) Predicting any arm movement feedback to induce three-dimensional illusory movements in humans. Neurophysiology 104:949-959 
EXERCISE AND SPORT PSYCHOLOGY 2

\section{SP2 K}

\section{The use of hypnosis with specific athletes hypnosis to improve sport performance in 4 cases}

\author{
N. Gava ${ }^{1}$ \\ ${ }^{1}$ Milton H. Erickson Institute, Turin
}

Purpose: The speech will describe, through the analysis of the key phases of 4 mental training programs, how hypnosis can be used with athletes who wish to enhance specific aspects of their physical performance, emotional balance and psychological state.

Methods: The author chose 4 cases of hypnotic mental training to describe the application of hypnotic techniques to enhance athletic performance.

Results: Case 1: Ipnosi in skiing. The case of a professional skier who asks for a mental training intervention to manage his fear of the slope, his anger and his impulsive behaviors that heavily hindered his run. The program will allow him to stabilize his performance which, before the intervention, often was unpredictable and unreliable. Case 2: Hypnosis in golf. The case of a golf player who, while performing very well while training, lost several matches because of his fear of the audience and performance anxiety. The speech will especially emphasize the importance of failure management on the field. Case 3: Hypnosis in basketball. This is the case of a basketball player who requested an intervention to improve his explosiveness on the court, to manage audience pressure and to regulate the problematic relationship with his teammates. Case 4: Hypnosis in rifle shooting. An example of how hypnosis can help athletes manage anxiety, time and physical pain in a professional shooter.

Conclusions: Hypnosis seems to be easily integrated in the training regime of different kinds of athletes. More research is needed to verify the effectiveness of such technique.

\section{References}

1. Weitzenhoffer AM (2000) The practice of hypnotism. Wiley, New York

2. Heap M, Aravind KAK (2002) Hartlands medical and dental hypnosis. Churchill Livingstone, London

3. De Benedittis (2016) L'ipnosi nel controllo del dolore. http://www.giuseppedebenedittis.it/lipnosi-nel-controllo-deldolore/

4. Liggett DR (2000) Enhancing imagery through hypnosis: a performance aid for athletes. Am J Clin Hypnosis 43(2):149-157. http://doi.org/10.1080/00029157.2000.10404267

5. Mizuguchi N, Nakata H, Uchida Y, Kanosue K (2012) Motor imagery and sport performance. J Phys Fitness Sports Med 1(1):103-111

6. Taktek K (2004) The effects of mental imagery on the acquisition of motor skills and performance: a literature review with theoretical implications. J Mental Imagery 28:79-114

7. Weinberg R (2008) Does imagery work? Effects on performance and mental skills. J Imagery Res Sport Phys Activ 3(1). http://doi.org/10.2202/1932-0191.1025

8. Pates J, Oliver R, Maynard I (2001) Effects of hypnosis on flow states and golf-putting performance. J Appl Sport Psychol 13(4):341-354. http://doi.org/10.2466/pms.2000.91.3f.1057

9. Robazza C, Bortoli L (1995) A case study of improved performance in archery using hypnosis. Percept Motor Skills
81(3 Pt 2):1364-1366. http://doi.org/10.2466/pms.1995.81.3f. 1364

10. Pates J, Palmi J (2002) The effects of hypnosis of flow states and performance. J Excell 6:48-62

11. Feltz DL, Short SE, Sullivan PJ (2008) Self-efficacy in sport. Human Kinetics, Champaign

12. Moritz SE, Feltz DL, Fahrbach KR, Mack DE (2000) The relation of self-efficacy measures to sport performance: a metaanalytic review. Res Q Exerc Sport 71(3):280-294. http://doi.org/10.1080/02701367.2000.10608908

13. Barker JB, Jones MV (2005) Using hypnosis to increase selfefficacy: a case study in elite judo. Sport Exerc Psychol Rev $1: 36-42$

14. Barker JB, Jones MV (2006) Using hypnosis, technique refinement, and self-modeling to enhance self-efficacy: a case study in cricket. Sport Psychol 20(1):94-110

15. Barker JB, Jones MV (2008) The effects of hypnosis on selfefficacy, affect, and soccer performance: a case study. Clin Sport Psychol 2:127-147

16. Barker J, Jones M, Greenles I (2010) Assessing the immediate and maintained effects of hypnosis on self-efficacy and soccer wall-volley performance. J Sport Exerc Psychol 32(2):243-52. http://www.ncbi.nlm.nih.gov/pubmed/20479480

17. Csikszentmihalyi M (1975) Beyond boredom and anxiety. Book Rev 703-707. http://doi.org/10.2307/2065805

18. Pates J, Cummings A, Maynard I (2002) The effects of hypnosis on flow states and three-point shooting performance in bastketball players. Sport Psychol 16(1):34-47. http://www.scopus. com/inward/record.url?eid=2-s2.0-

0142167198\&partnerID $=40 \& \mathrm{md} 5=2 \mathrm{ab} 152744907 \mathrm{e} 0 \mathrm{fa} 7 \mathrm{cf} 93 \mathrm{ba} 0$ $01 \mathrm{bf6e} 81$

\section{SP2 O}

Hypnosis and self-hypnosis in the improvement of sport performance: a review of the scientific literature

\author{
N. Gava ${ }^{1}$, M. Cavarra $^{2}$ \\ ${ }^{1}$ Milton H. Erickson Institute, Turin, \\ ${ }^{2}$ Milton H. Erickson Institute, Turin
}

Purpose: The presentation will list, organize and critically comment the scientific, published, peer reviewed papers that exploring the effectiveness of hypnosis in improving the variables related to sports performance.

Methods: We performed a comprehensive database search (Pubmed, Scopus, Psychinfo) of the scientific literature since 1995 until 2018 using the keywords "sport hypnosis".

Results: Sport psychology increasingly attributes importance to mental training of athletes. Mental imagery, self-efficacy, relaxation and flow states are among the most important variables to determine the outcome of athletic performance.

Conclusions: Results indicate that athletes who train with hypnosis show improvements in the quality of their performance. While promising, these works emphasize the need for further research and greater methodological rigor in order to more solidly investigate the effectiveness of such approach to mental training. Finally, a case of an athlete who was trained with hypnosis will be described and discussed.

\section{References}

1. Weitzenhoffer AM (2000) The practice of hypnotism. Wiley, New York 
2. Heap M, Aravind KAK (2002) Hartlands medical and dental hypnosis. Churchill Livingstone, London

3. De Benedittis (2016). L'ipnosi nel controllo del dolore. http://www.giuseppedebenedittis.it/lipnosi-nel-controllo-deldolore/

4. Liggett DR (2000) Enhancing imagery through hypnosis: a performance aid for athletes. Am J Clin Hypnosis 43(2):149-157. http://doi.org/10.1080/00029157.2000.10404267

5. Mizuguchi N, Nakata H, Uchida Y, Kanosue K (2012) Motor imagery and sport performance. The J Phys Fitness Sports Med 1(1):103-111

6. Taktek K (2004) The effects of mental imagery on the acquisition of motor skills and performance: A literature review with theoretical implications. J Mental Imagery 28:79-114.

7. Weinberg R (2008) Does imagery work? Effects on performance and mental skills. J Imagery Res Sport Phys Activ 3(1). http://doi.org/10.2202/1932-0191.1025

8. Pates J, Oliver R, Maynard I (2001) Effects of hypnosis on flow states and golf-putting performance. J Appl Sport Psychol 13(4):341-354. http://doi.org/10.2466/pms.2000.91.3f.1057

9. Robazza C, Bortoli L (1995) A case study of improved performance in archery using hypnosis. Percept Motor Skills 81(3 Pt 2):1364-6. http://doi.org/10.2466/pms.1995.81.3f.1364

10. Pates J, Palmi J (2002) The effects of hypnosis of flow states and performance. J Excell 6:48-62

11. Feltz DL, Short SE, Sullivan PJ (2008) Self-efficacy in sport. Human Kinetics, Champaign

12. Moritz SE, Feltz DL, Fahrbach KR, Mack DE (2000) The relation of self-efficacy measures to sport performance: a metaanalytic review. Res Q Exerc Sport 71(3):280-294. http://doi.org/10.1080/02701367.2000.10608908

13. Barker JB, Jones MV (2005) Using hypnosis to increase selfefficacy: a case study in elite judo. Sport Exerc Psychol Rev $1: 36-42$

14. Barker JB, Jones MV (2006) Using hypnosis, technique refinement, and self-modeling to enhance self-efficacy: a case study in cricket. Sport Psychol 20(1):94-110

15. Barker JB, Jones MV (2008) The effects of hypnosis on selfefficacy, affect, and soccer performance: a case study. Clin Sport Psychol 2:127-147

16. Barker J, Jones M, Greenles I (2010) Assessing the immediate and maintained effects of hypnosis on self-efficacy and soccer wall-volley performance. J Sport Exerc Psychol 32(2):243-252. http://www.ncbi.nlm.nih.gov/pubmed/20479480

17. Csikszentmihalyi M (1975) Beyond boredom and anxiety. Book Reviews, pp 703-707. http://doi.org/10.2307/2065805

18. Pates J, Cummings A, Maynard I (2002) The effects of hypnosis on flow states and three-point shooting performance in bastketball players. Sport Psychol 16(1):34-47. http://www.scopus. com/inward/record.url?eid=2-s2.0-0142167198\& partnerID $=40 \&$ md5=2ab152744907e0fa7cf93ba001bf6e 81

\section{SP2 O}

\section{The inattentional blindness in soccer referees}

\author{
R. Pazzona ${ }^{1}$, M. Guicciardi ${ }^{1}$, M. Murgia ${ }^{2}$ D. Grassi ${ }^{3}$
}

${ }^{1}$ Department of Psychology, University of Cagliari, Cagliari, Italy; ${ }^{2}$ Department of Life Sciences, University of Trieste, Trieste, Italy;

${ }^{3}$ Università Telematica San Raffaele Roma

Purpose: Inattentional blindness is the inability to detect unexpected events occurring in the visual field of an individual. This phenomenon, which became famous after the experiments of Simon and Chabris (1999) on the invisible gorilla, has found little use in sport psychology so far. This study aims at verifying whether soccer referees, in comparison to other participants, are more able to notice unexpected events, because they usually have to identify and cope with unexpected situations during matches.

Methods: The experiment involved referees, referees' observers, and university students. The participants were engaged in an attentional task which could have two levels of complexity: easy and difficult.

Results: The results show that referees have a superior capacity to identify unexpected events compared to the other participants. Although the referees were less experienced than referees' observers, they notice the unexpected event more frequently, regardless of the difficulty level of the task.

Conclusions: Overall, the easier task led to a higher rate of identification of the unexpected event, but further studies are needed to improve the standardization of stimuli.

\section{Reference}

Pazzona R, Guicciardi M, Murgia M (2018) The inattentional blindness in soccer referees. Med Sport 71:216-225

\section{SP2 O}

Executive functioning profiles in elite volleyball athletes: preliminary results by a sport-specific task switching protocol

S. Montuori ${ }^{1}$, G. D'Aurizio ${ }^{2}$, P. Sorrentino ${ }^{3}$, F. Foti ${ }^{4,5}$ $\overline{\text { M. Liparoti }}^{1}$, A. Lardone ${ }^{1}$, M. Pesoli ${ }^{1}$, G. Sorrentino ${ }^{1,6,7}$, G. Curcio $^{2}$, L. Mandolesi ${ }^{1,5}$ *

${ }^{I}$ Department of Movement Sciences and Wellbeing, University "Parthenope", NA, Italy;

${ }^{2}$ Department of Biotechnological and Applied Clinical Sciences, University of L'Aquila, $A Q$, Italy;

${ }^{3}$ Department of Engineering, University "Parthenope", NA, Italy;

${ }^{4}$ Department of Medical and Surgical Sciences, "Magna Graecia" University of Catanzaro, CZ, Italy;

${ }^{5}$ IRCCS Fondazione Santa Lucia, RM, Italy;

${ }^{6}$ Istituto di Diagnosi e Cura Hermitage Capodimonte, NA, Italy; ${ }^{7}$ Institute of Applied Sciences and Intelligent Systems, CNR, Pozzuoli (NA), Italy

Purpose: Executive functions (EF) are crucial for the athletes' success, and they are even more essential in open skill sports (e.g. volleyball and football). In these sports, due to continuously changing conditions, goal-directed behaviours need to be repeatedly adjusted and corrected. One of the most important EF is the ability to continuously switch between two different tasks that are asked to be performed in random sequence. We used a taskswitching protocol in 
elite volleyball athletes, usually playing different roles, with the aim of identify a sort of "executive style".

Methods: Twenty-seven elite volleyball athletes, divided into three groups (Strikers; Defenders; Mixed) completed a customized sportspecific task-switching paradigm.

Results: Data evidenced that each role has specific characteristics: Strikers group resulted to be the fastest to answer to stimuli, while Defenders group provided a worsened performance particularly when defensive actions, that probably requires more cognitive elaborations, had to be processed. Different effects emerged from of the Mixed group, the one with more expertise, that appeared more accurate in the responses. These results evidenced a minimal degree of cognitive flexibility for highly specialized Strikers and a maximum level for Mixed, allowing thus to highlight specific profiles of athletes.

Conclusions: Data observed will permit to develop a test that analyzes the executive profile during the recruitment in a team, revealing a useful tool for choosing the most suitable role.

\section{References}

Memmert D (2011) Creativity, expertise, and attention: exploring their development their relationships. J Sports Sci 29(1):93-102

Vestberg T, Gustafson R, Maurex L, Ingvar M, Petrovic P (2012)

Executive functions predict the success of top-soccer players. PLoS One 7(4) 